Florida International University FIU Digital Commons

\title{
Nanomaterial-Based Electrochemical and Colorimetric Sensors for On-Site Detection of Small-Molecule Targets
}

Bhargav Guntupalli

Florida International University, bgunt001@fiu.edu

DOI: 10.25148 /etd.FIDC001906

Follow this and additional works at: https://digitalcommons.fiu.edu/etd

Part of the Analytical Chemistry Commons

\section{Recommended Citation}

Guntupalli, Bhargav, "Nanomaterial-Based Electrochemical and Colorimetric Sensors for On-Site Detection of Small-Molecule Targets" (2017). FIU Electronic Theses and Dissertations. 3488.

https://digitalcommons.fiu.edu/etd/3488

This work is brought to you for free and open access by the University Graduate School at FIU Digital Commons. It has been accepted for inclusion in FIU Electronic Theses and Dissertations by an authorized administrator of FIU Digital Commons. For more information, please contact dcc@fiu.edu. 


\title{
FLORIDA INTERNATIONAL UNIVERSITY
}

Miami, Florida

\section{NANOMATERIAL-BASED ELECTROCHEMICAL AND COLORIMETRIC \\ SENSORS FOR ON-SITE DETECTION OF SMALL-MOLECULE TARGETS}

\author{
A dissertation submitted in partial fulfillment of \\ the requirements for the degree of \\ DOCTOR OF PHILOSOPHY \\ in \\ CHEMISTRY \\ by \\ Bhargav Guntupalli
}


To: Dean Michael R. Heithaus

College of Arts, Sciences and Education

This dissertation, written by Bhargav Guntupalli, and entitled Nanomaterial-Based Electrochemical and Colorimetric Sensors for On-Site Detection of Small-Molecule Targets, having been approved in respect to style and intellectual content, is referred to you for judgment.

We have read this thesis and recommend that it be approved.

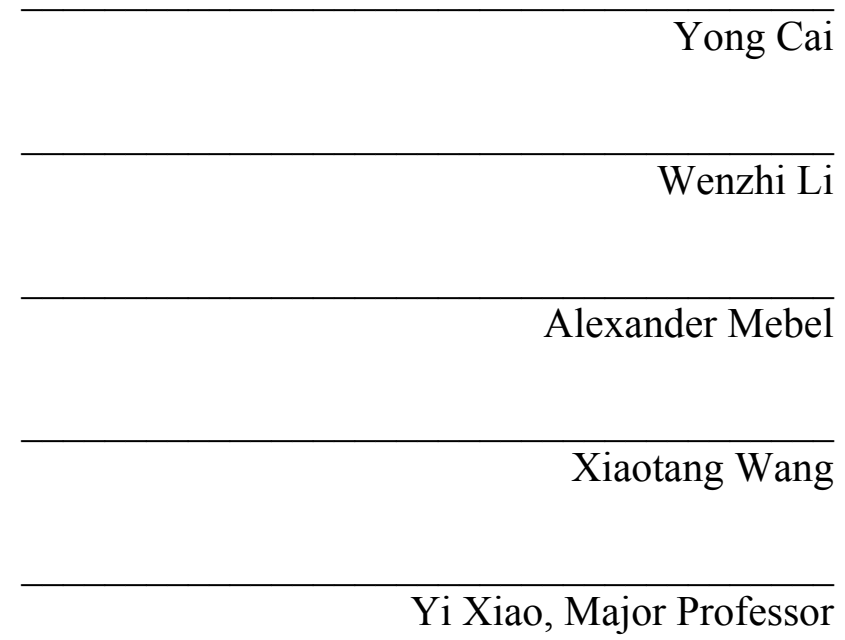

Date of Defense: April 20, 2017

The dissertation of Bhargav Guntupalli is approved.

Dean Michael R. Heithaus

College of Arts, Sciences and Education

Andrés G. Gil

Vice President for Research and Economic Development and Dean of the University Graduate School

Florida International University, 2017 
C Copyright 2017 by Bhargav Guntupalli

All rights reserved. 


\section{ACKNOWLEDGMENTS}

I would first and foremost like to thank my major professor Dr. Yi Xiao of the Department of Chemistry and Biochemistry at Florida International University. The door to Dr. Xiao's office was always open whenever I ran into a trouble spot or had a question about my research or writing. She consistently allowed this research work to be my work but steered me in the right the direction whenever she thought I needed it.

I would also like to thank the expertise of my research committee who were instrumental in providing support, feedback, and guidance throughout my stay at this university. I am grateful to Dr. Alexander Mebel, Dr. Wenzhi Li, Dr. Yong Cai, and Dr. Xiaotang Wang for their willingness to serve on my dissertation committee and their valuable input, advice time and again. Without their passionate participation the dissertation could not have been successfully accomplished. I would also like to acknowledge all the contributors involved in my publications from different departments and universities whose contributions made my research work shine brighter. I would like to thank my lab mates and postdocs past and present for their feedback and support, which helped my personal and professional development.

I would like to acknowledge Department of Chemistry and Biochemistry and Dissertation Year Fellowship from University Graduate School at Florida International University for the financial support.

Finally, I must express my very profound gratitude to my parents Sambasiva Rao and Padma Guntupalli and my sister Harsha Surapaneni for providing me with unfailing support and continuous encouragement throughout my years of study and through the 
process of researching and writing this thesis. This accomplishment would not have been possible without them. 


\begin{abstract}
OF THE DISSERTATION
NANOMATERIAL-BASED ELECTROCHEMICAL AND COLORIMETRIC SENSORS FOR ON-SITE DETECTION OF SMALL-MOLECULE TARGETS

by Bhargav Guntupalli
\end{abstract}

Florida International University, 2017

Miami, Florida

Professor Yi Xiao, Major Professor

An ideal biosensor is a compact and in-expensive device that is able to readily and rapidly detects different types of analytes with high sensitivity and specificity. The affectability of a biosensing methodology is subject to the limit of nanomaterials to transduce the target binding process to an improved perceptible signal, while the selectivity is accomplished by considering the binding and specificity of certain moieties to their targets. Keeping these requirements in mind we have chosen nanomaterials such as carbon nanotubes (CNTs) and gold nanoparticles (AuNPs) that has catalytic properties combined with their size, shape and configuration dependent chemical and physical properties as essential precursors and signaling components for creation of biosensors with tremendous sensitivity. The primary goal of the research work described in this dissertation is to develop and evaluate novel methods to detect various analytes using nanomaterials, at the same time making an affordable architecture for point-of-care (POC) applications. We report here in chapter 3 a simple and new strategy for preparing disposable, paper-based, porous AuNP/M-SWCNT hybrid thin gold films with high conductivity, rapid electron transfer rates, and excellent electrocatalytic properties to 
achieve multiple analyte electrochemical detection with a resolution that greatly exceeds that of purchased flat gold slides. We further explored the use of nanomaterial-based paper films in more complex matrices to detect analytes such as NADH, which can act as a biomarker for certain cellular redox imbalances and disease conditions. Carbon nanotubes with their large activated surfaces and edge-plane sites (defects) that are ideal for performing NADH oxidation at low potentials without any help of redox mediators minimizing surface fouling in complex matrices is described in chapter 4 . With an instrument-free approach in mind we further focused on a colorimetric platform using split cocaine aptamers and gold nanoparticles (AuNPs) to detect cocaine for on-site applications as described in chapter 5. In chapter 5, the split aptamer sequences were evaluated mainly on three basic criteria, the hybridization efficiency, specificity towards the analyte (cocaine), and the reaction time to observe a distinguishable color change from red to blue. The assay is an enzyme-assisted target recycling (EATR) strategy following the principle that nuclease enzyme recognizes probe-target complexes, cleaving only the probe strand releasing the target for recycling. We have also studied the effect of the number of binding domains with variable chain lengths on either side of the apurinic (AP) site. On the basis of our results, we finally shortlisted the sequence combination with maximum signal enhancement fold which is instrumental in development of colorimetric platform with faster, and specific reaction to observe a distinctive color change in the presence of cocaine. 


\section{TABLE OF CONTENTS}

CHAPTER $\quad$ PAGE

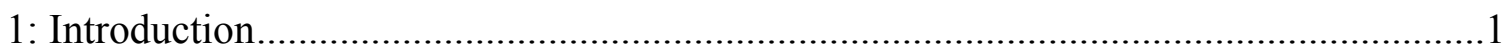

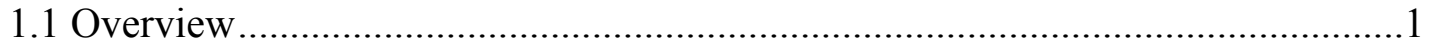

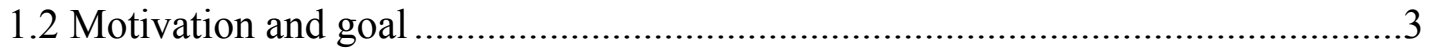

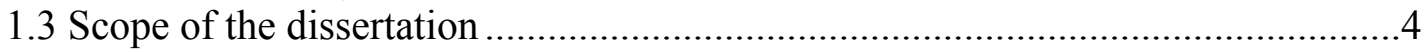

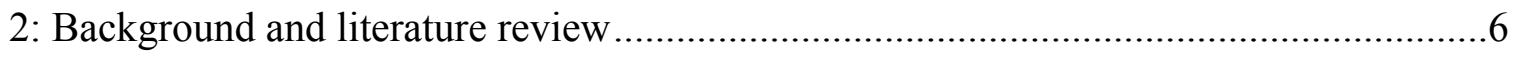

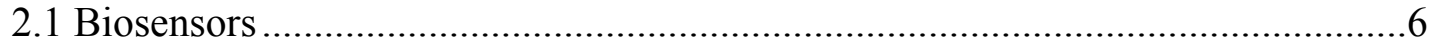

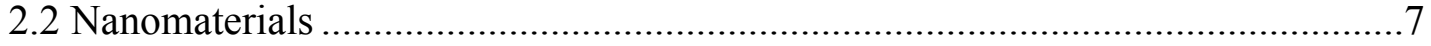

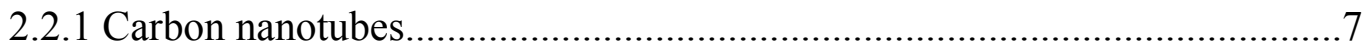

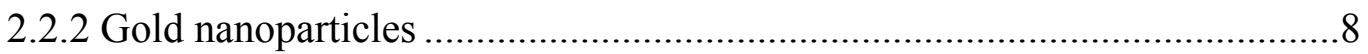

2.2.2.1 Mechanism of AuNP synthesis .......................................................

2.2.2.2 Factors governing AuNP size and dispersion .................................10

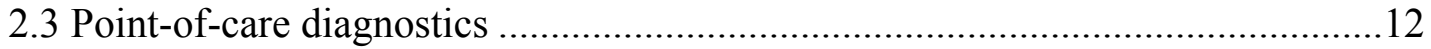

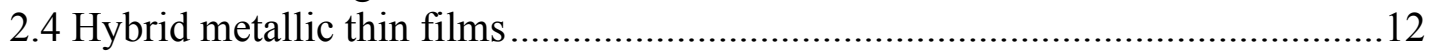

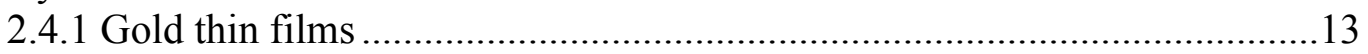

2.4.2 Paper as substrate ...................................................................................

2.4.3 Paper-based gold thin films ..................................................................... 14

2.5 Electrochemical detection of dopamine and serotonin ......................................15

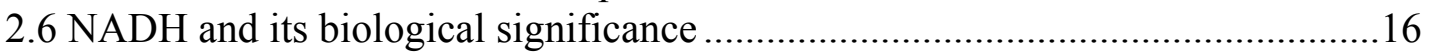

2.6.1 Current methods for detecting NADH in biosamples and cell extracts......17

2.6.2 In vitro applications of NADH ................................................................. 18

2.6.3 Electrochemical detection of NADH in complex samples ..........................19

2.6.3.1 CNT-based electrochemical detection of NADH ..........................20

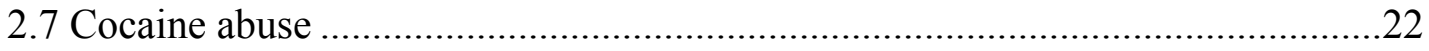

2.7.1 Current methods for presumptive screening of cocaine ……......................23

2.7.2 Aptamers as biosensor recognition elements ..............................................24

2.7.3 Colorimetric AuNP-conjugated aptamer sensors........................................25

2.7.4 Aptamer-based biosensors for cocaine detection.........................................26

2.7.5 Cooperative binding split aptamers .........................................................28

3: Ambient Filtration Method to Rapidly Prepare Highly Conductive, Paper-Based Porous Gold Films for Electrochemical Biosensing ................................................30

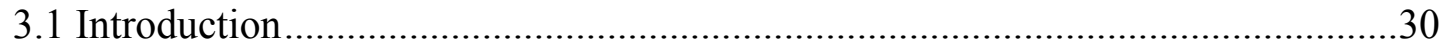

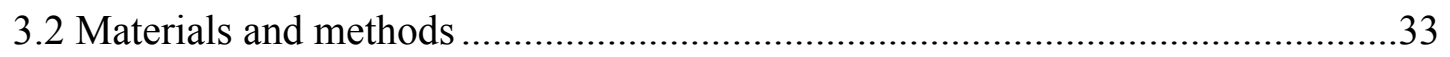

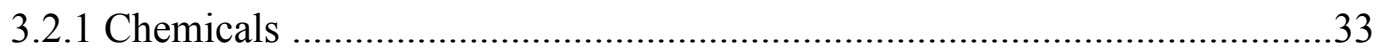

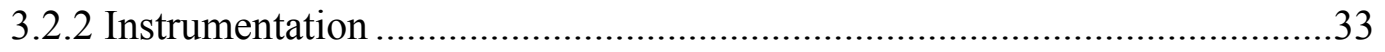

3.2.3 Preparation of colloidal gold nanoparticle solution........................................35

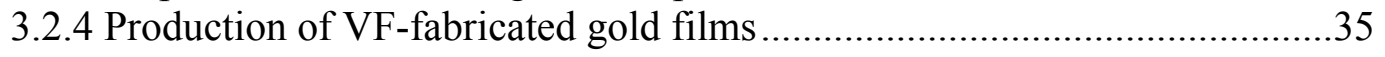

3.2.5 Alternative VF-fabricated gold film compositions .....................................36

3.2.6 Template-free electrodeposition of AuNPs on paper-based M-SWCNT 
film

3.2.7 Hydrogen bubble-directed electrodeposition of AuNPs on paper-based

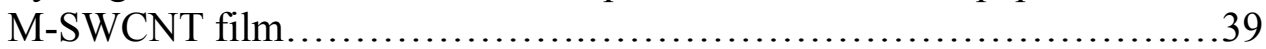

3.2.8 Contact angle measurements on various surfaces.....................................39

3.2.9 Determination of surface roughness of fabricated gold films using cyclic voltammetry ............................................. 40

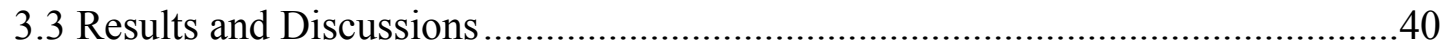

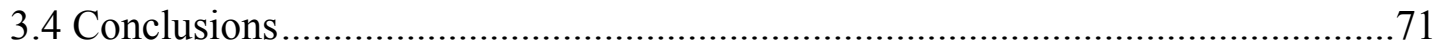

4: Paper-Based SWCNT Thin Film Electrode for Drug Detection and Disease

Diagnosis - Electrocatalytic Detection of NADH in Complex Matrices ......................73

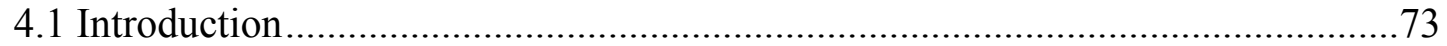

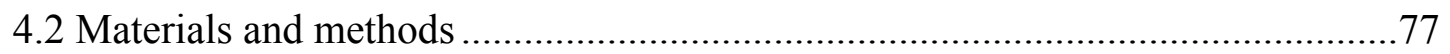

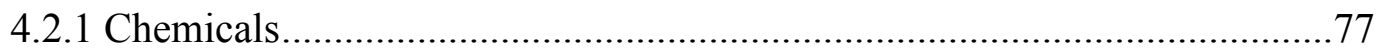

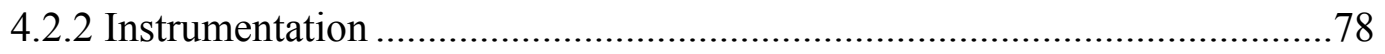

4.2.3 Preparation of paper-based P-SWCNT film ...........................................79

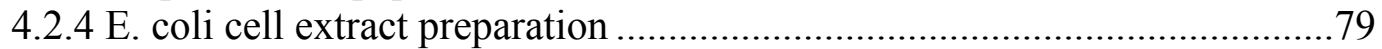

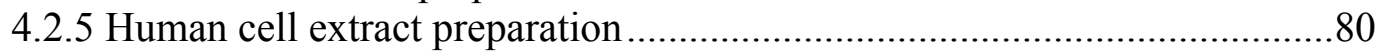

4.2.6 Saliva collection and preparation of the pooled saliva matrix ....................81

4.2.7 EMIT assay and electrochemical detection of NADH production in saliva

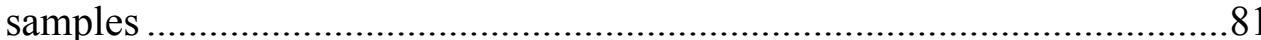

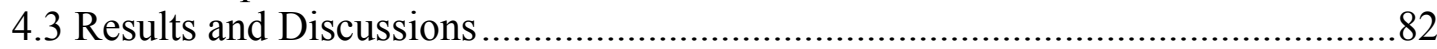

4.3.1 Effect of amount of P-SWCNT on conductivity and electron transfer rate

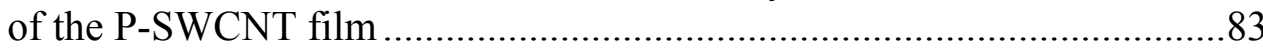

4.3.2 Effect of the amount of P-SWCNT on NADH electrocatalytic responses in PBS buffer

4.3.3 Effect of the amount of P-SWCNT on NADH electrocatalytic responses

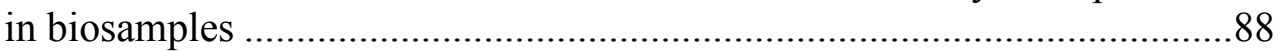

4.3.4 Characterization of surface morphology of paper-based P-SWCNT film...90

4.3.5 Paper-based P-SWCNT film electrodes for detection of enzyme-generated

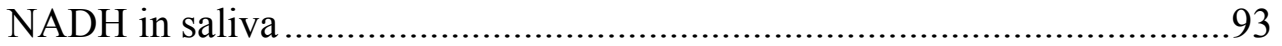

4.4 Conclusions

5: Evolution of Probe Sequence Design Using Split Aptamers to Facilitate Efficient Enzyme Amplified Target Recycling for Onsite Colorimetric Detection of Cocaine.

5.1 Introduction

5.2 Materials and methods

5.2.1 Thiolated short aptamer fragments

5.2.2 Long aptamer fragments

5.2.3 Synthesis of AuNPs

5.2.4 Modification of thiolated short aptamer fragment on AuNPs

5.2.5 Characterization of short aptamer fragment density on the surface of AuNP before and after DTT-displacement....

5.2.6 Effect of the surface density of short aptamer fragment modified AuNPs on Exo III-assisted cocaine recycling 


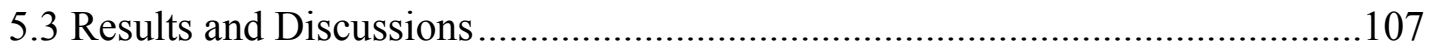

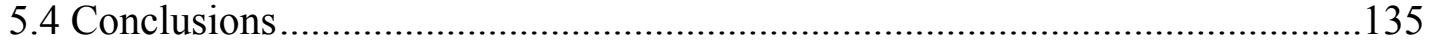

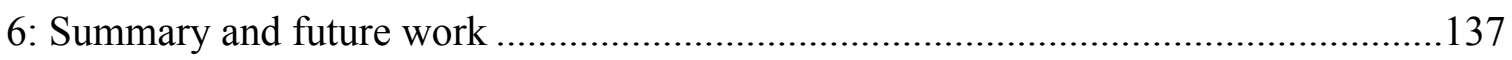

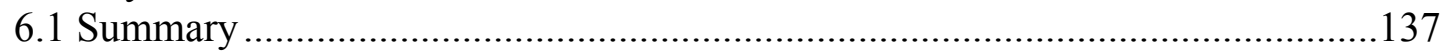

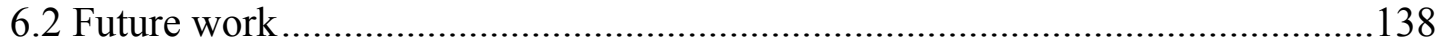

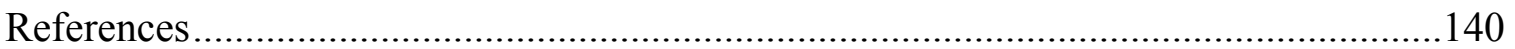

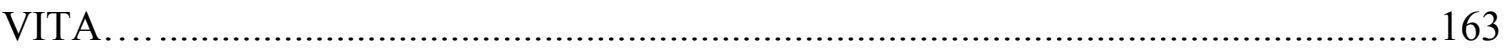




\section{LIST OF FIGURES}

FIGURE

PAGE

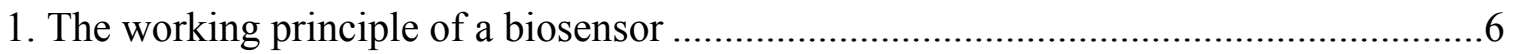

2. Schematic illustration of the preparation of working VF-fabricated gold film electrodes

3. Effect of pore-size of MCE filter papers on sheet resistance and electron transfer

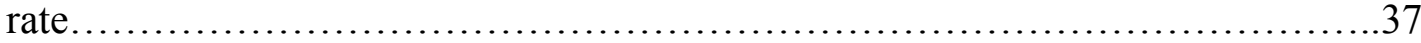

4. Transmission electron microscopy (TEM) image of (A) citrate-capped AuNPs (B) Frequency distribution histogram...........................................................................41

5. Porous thin gold film preparation via vacuum filtration. 42

6. X-ray energy dispersive spectroscopy (EDS) of (A) a single layer of M-SWCNTs on MCE or (B) an AuNP/M-SWCNT hybrid film on MCE

7. Apparent contact angle of water $(\theta \mathrm{w}, \mathrm{a})$ for the surface of various films.

8. Effect of variable amounts of M-SWCNTs on sheet resistance in the absence of AuNPs.

9. Effect of variable amounts of M-SWCNTs on sheet resistance in the presence of 35 picomoles AuNPs

10. Effects of variable amounts of AuNPs on sheet resistance with fixed amount of MSWCNTs

11. Effect of variable amounts of M-SWCNTs on electron transfer rate with fixed amount of AuNPs (35 pmoles)

12. Front angle view (top) and side angle view (bottom) of films fabricated with 35 pmoles AuNPs and variable amounts of M-SWCNTs

13. Effects of variable amounts of P-SWCNTs on sheet resistance and electron transfer rate.

14. Effects of variable amounts of AuNPs on electron transfer rate.

15. Front (top) and side angle view (bottom) of films fabricated with $8 \mu \mathrm{g}$ M-SWCNTs and varying amounts of AuNPs. 
16. Effect of AuNP size on sheet resistance and electron transfer rates. (A) Sheet resistance comparison of VF-fabricated gold films (B) Electron transfer rate comparison of the same films.....

17. Surface morphology as characterized by atomic force microscopy (AFM). Typical AFM images of (A) MCE filter paper, (B) M-SWCNT-loaded MCE paper, (C) VFfabricated AuNP/M-SWCNT film, (D) AuNP-loaded MCE paper, and (E) a commercially-purchased gold slide. (F) Surface roughness measurements for the various films shown in A-E

18. AFM images of (A) the AuNP/M-SWCNT hybrid film prepared with $8 \mu \mathrm{g}$ MSWCNTs and 35 pmole AuNPs. (B) The frequency distribution histogram of the AuNP/M-SWCNT hybrid film.

19. AFM image of the edge of a VF-fabricated gold film prepared with $8 \mu \mathrm{g}$ M-SWCNTs and 35 pmole AuNPs.

20. Surface morphology of various films as characterized by SEM. SEM images of (A) MCE filter paper, (B) M-SWCNT-loaded MCE paper, and (C) AuNP/M-SWCNT hybrid film on MCE paper

21. Cross-sectional SEM images of our hybrid gold films. (A) A comprehensive view of the cross-section of the paper-based hybrid gold film. (B) An interior view of MCE paper and nanomaterial interfacial layer

22. Comparison of the electrochemical characteristics of a flat gold slide relative to our VF-fabricated porous gold film

23. Comparison of the electrochemical characteristics of a flat gold slide relative to our VF-fabricated porous gold film

24. Comparison of the electrochemical responses of our VF-fabricated gold film relative to other films....

25. Cyclic voltammetric responses of various concentrations of DA in $0.1 \mathrm{M} \mathrm{PBS}(\mathrm{pH}$ 7.4)

26. Cyclic voltammetric responses of various concentrations of 5-HT in 0.1 M PBS $(\mathrm{pH} 7.4)$

27. Electrodes constructed from our hybird gold (A) reproducibility in small molecule detection. (B) Stability of our hybrid gold film electrodes over a period of two weeks.

28. Effect of surface cleaning treatment on our hybrid gold film on electron transfer rate. 
29. Effect of solution soak time on electron transfer rate for our hybrid gold films.

30. Effect of $\mathrm{pH}$ on electron transfer rate of our hybrid gold film.

31. Effect of temperature on electron transfer rate of our hybrid gold film

32. Comparison of electrocatalytic response of DA on various paper-based gold films...67

33. Comparison of electrochemical characteristics and roughness factors of bare, flat gold slides, TF-deposited gold film, HB-directed gold film and VF-fabricated gold film

34. Comparison of simultaneous target detection with various gold films.

35. Preparation of Porous P-SWCNT film via vacuum filtration .82

36. Effect of variable amounts of P-SWCNTs on sheet resistance of the fabricated film. 84

37. Effect of variable amounts of P-SWCNTs on electron transfer rate

38. Effect of amount of P-SWCNT on NADH electrocatalytic responses in PBS buffer Cyclic voltammograms of P-SWCNT films in $0.1 \mathrm{M}$ PBS.

39. Comparison of the electrochemical catalytic responses of our various paper-based P-SWCNT films in $0.1 \mathrm{M}$ PBS buffer using cyclic voltammetry

40. Comparison of the electrochemical responses of our various paper-based P-SWCNT film electrodes in pooled saliva using cyclic voltammetry.

41. Cyclic voltammograms of $50 \mu \mathrm{g}$ P-SWCNT film in 50\% saliva in the presence (red trace) and absence (black trace) of $1 \mathrm{mM} \mathrm{NADH.}$

42. Surface morphology of our $50 \mu \mathrm{g}$ P-SWCNT loaded film as characterized by atomic force microscopy (AFM)

43. Effect of E. coli cell extract dilution for NADH oxidation on P-SWCNT electrode ..92

44. Differential pulse voltammograms of $50 \mu \mathrm{g}$ P-SWCNT film in un-diluted human cell extract after dialysis from normal (red trace) and FRDA patient (black trace) lymphoblasts

45. Effect of Pooled saliva dilution for NADH oxidation on P-SWCNT electrode obtained from DPV

46. TECAN spectrophotometer response for the enzyme-produced NADH as the concentration of benzoylecgonine increased from $5 \mathrm{ng} / \mathrm{mL}$ to $1000 \mathrm{ng} / \mathrm{mL}$ 
47. A similar hyperbolic response was observed for the enzyme-produced NADH as the concentration of benzoylecgonine increased from $5 \mathrm{ng} / \mathrm{mL}$ to $1000 \mathrm{ng} / \mathrm{mL}$

48. Concentration and dispersion of synthesized AuNPs. (A) UV-Vis spectrum of the synthesized AuNPs (B) Particle size distribution measurement using dynamic light scattering....

49. Transmission electron microscopy (TEM) image of (A) citrate-capped AuNPs (B) Frequency distribution histogram shows an average particle diameter

50. Formation of folded split aptamers with single binding domain (SSA) from its single cocaine-binding parent aptamer (38GC).

51. AuNP-reported, Exo III-assisted colorimetric detection of cocaine. (A) Schematic principle of the assay. (B) UV-vis spectra of the short fragment-modified AuNPs in the absence (black trace) and presence (red trace) of cocaine.

52. Effect of DTT concentration on the surface coverage of SF-SSA resulting in change of Exo III kinetics for cocaine detection

53. Calculation of surface coverage of SF-SSA-modified AuNPs treated with different concentrations of DTT from $0 \mu \mathrm{M}$ to $1200 \mu \mathrm{M}$ using Oligreen assay (Quant-iT ${ }^{\mathrm{TM}}$ OliGreen ${ }^{\circledR}$ ssDNA Reagent)

54. Effect of the LF-525 concentration on the efficiency of target-aptamer assembly and Exo III kinetics for cocaine detection

55. Effect of LF-925 concentration on the efficiency of target-aptamer assembly and Exo III kinetics for cocaine detection

56. Effect of LF-725 concentration on the efficiency of target-aptamer assembly and Exo III kinetics for cocaine detection

57. Effect of LF-225 concentration on the efficiency of target-aptamer assembly and Exo III kinetics for cocaine detection

58. Effect of LF-425 concentration on the efficiency of target-aptamer assembly and Exo III kinetics for cocaine detection

59. Utilizing SSA-925 to derivate CBSA-5325

60. Effect of LF-CBSA-5325 concentration on the efficiency of target-aptamer assembly and Exo III kinetics for cocaine detection

61. Utilizing CBSA-5325 to engineer CBSA-5345 
62. Utilizing CBSA-5325 to engineer CBSA-5335

63. Effect of DTT concentration on the surface coverage of SF-CBSA-5345 resulting in change of Exo III kinetics for cocaine detection

64. Effect of LF-CBSA-5345 concentration on the efficiency of target-aptamer assembly and Exo III kinetics for cocaine detection

65. Effect of DTT concentration on the surface coverage of SF-CBSA-5335 resulting in change of Exo III kinetics for cocaine detection

66. Effect of LF-CBSA-5335 concentration on the efficiency of target-aptamer assembly and Exo III kinetics for cocaine detection ......

67. Utilizing CBSA-5335 to engineer CBSA-5335-10T

68. Effect of DTT concentration on the surface coverage of SF-CBSA-5335-10T resulting in change of Exo III kinetics for cocaine detection

69. Effect of LF-CBSA-5335 concentration on the efficiency of target-aptamer assembly and Exo III kinetics for cocaine detection 


\section{LIST OF ABBREVIATIONS}

\begin{tabular}{|c|c|}
\hline Abs & absorbance \\
\hline $\mathrm{AgNO}_{3}$ & silver nitrate \\
\hline AP & apurinic endonucleolytic \\
\hline ATR-IR & attenuated total reflectance - infrared \\
\hline $\mathrm{AuCl}$ & gold chloride \\
\hline AuNP & gold nanoparticle \\
\hline$\beta$ & beta \\
\hline BCNT & boron doped carbon nanotube \\
\hline CBSA & cooperative binding split aptamer \\
\hline $\mathrm{CE}$ & capillary electrophoresis \\
\hline $\mathrm{CL}$ & chemiluminescence \\
\hline $\mathrm{cm}$ & centimeter \\
\hline CNS & central nervous system \\
\hline CNT & carbon nanotube \\
\hline $\mathrm{Coc}$ & cocaine \\
\hline $\mathrm{CP}$ & capture probe \\
\hline CVD & chemical vapor deposition \\
\hline$\delta$ & delta \\
\hline $\mathrm{oC}$ & degrees Celsius \\
\hline DA & dopamine \\
\hline DCIP & 2-6-dichloroindophenol \\
\hline D] & deionized water \\
\hline
\end{tabular}




\begin{tabular}{|c|c|}
\hline DLS & dynamic light scattering \\
\hline DNA & deoxyribonucleic acid \\
\hline DNA-AuNPs & DNA modified gold nanoparticles \\
\hline dsDNA & double-stranded DNA \\
\hline DTT & dithiothreitol \\
\hline EATR & enzyme-assisted target recycling \\
\hline ELISA & enzyme-linked immunosorbent assay \\
\hline EMIT & enzyme multiplied immunoassay technique \\
\hline Exo III & exonuclease III \\
\hline FDA & food and drug administration \\
\hline FPIA & fluorescence polarization immunoassay \\
\hline FRET & fluorescence resonance energy transfer \\
\hline $\mathrm{g}$ & gram \\
\hline GCE & glassy carbon electrode \\
\hline GC/MS & gas chromatography/mass spectrometry \\
\hline GDH & glucose dehydrogenase \\
\hline GO & graphene oxide \\
\hline GOX & glucose oxidase \\
\hline $\mathrm{h}$ & hour \\
\hline HB & hydrogen bubble \\
\hline HIV & human immunodeficiency virus \\
\hline HPLC & high-performance liquid chromatography \\
\hline ITO & indium tin oxide \\
\hline
\end{tabular}




\begin{tabular}{|c|c|}
\hline $\mathrm{K}_{\mathrm{D}}$ & dissociation constant \\
\hline $\mathrm{KD}$ & kilo dalton \\
\hline $\mathrm{L}$ & liter \\
\hline $\mathrm{LF}$ & long fragment \\
\hline$\lambda$ & lambda \\
\hline $\mathrm{m}$ & milli \\
\hline M & moles per liter \\
\hline MIPs & molecular imprinted polymers \\
\hline M-SWCNT & metallic single-walled carbon nanotube \\
\hline MB & methylene blue \\
\hline MCE & mixed cellulose ester \\
\hline $\mathrm{MCH}$ & 6-Mercapto-1-hexanol \\
\hline$\mu$ & micro \\
\hline $\min$ & minute \\
\hline mol & mole \\
\hline MS & mass spectrometry \\
\hline $\mathrm{NADH}$ & dihydronicotinamide adenine dinucleotide \\
\hline $\mathrm{NAD}^{+}$ & nicotinamide adenine dinucleotide \\
\hline $\mathrm{nM}$ & nanomolar \\
\hline $\mathrm{nm}$ & nanometer \\
\hline NSDUH & national survey on drug use and health \\
\hline$\%$ & percentage \\
\hline p-ATP & p-aminobenzenethiol \\
\hline
\end{tabular}




\begin{tabular}{|c|c|}
\hline POC & point of care \\
\hline P-SWCNT & puretube single-walled carbon nanotube \\
\hline RIA & radioimmunoassay \\
\hline RT & room temperature \\
\hline $\mathrm{s}$ & second \\
\hline SADC & sodium acetone dicarboxylate \\
\hline SAM & self assembled monolayers \\
\hline SERS & surface-enhanced raman scattering \\
\hline $\mathrm{SiO}_{2}$ & silicon dioxide \\
\hline SPR & surface plasmon resonance \\
\hline SSA & structure switching aptamer \\
\hline ssDNA & single-stranded DNA \\
\hline TEM & transmission electron microscopy \\
\hline $\mathrm{TF}$ & template free \\
\hline THMS & triple-helix molecular switch \\
\hline Tris & tris(hydroxymethyl)aminomethane \\
\hline UV-Vis & ultraviolet-visible \\
\hline VF & vacuum filtration \\
\hline $\mathrm{w} /$ & with \\
\hline w/o & without \\
\hline 5-HT & serotonin \\
\hline
\end{tabular}




\section{CHAPTER 1: Introduction}

\subsection{Overview}

There is a strong demand for analytical sensors that can perform quick and accurate analyses for applications in areas such as disease diagnosis, forensics, environmental protection, drug development, and hygiene. Considerable effort has been invested into the development of biosensors, which are devices containing biological elements capable of recognizing specific target molecules. These recognition events are subsequently transduced at the detector element into a semi-quantitative or quantitative signal that is proportional to the concentration of the analyte. The choice of materials employed in the fabrication of such biosensors is a crucial factor in determining their utility and suitability for accurate on-site detection. Similarly, the selection of an appropriate transduction process can minimize or even eliminate the need for expensive instrumentation or expertise to obtain a quick and accurate analysis. Biosensors, therefore, offer the potential to achieve scaled-down, inexpensive, label-free analysis with limited sample preparation requirements, making them potentially ideal for on-site detection applications. Electrochemical sensors are especially well suited for dealing with real-world sample matrices without the need for time-consuming and labor-intensive pre-treatment steps. These devices offer advantages that include excellent repeatability and accuracy, a wide linear response range, low detection limits, and the capacity for real-time measurements. Electrochemical sensors constructed from nanomaterials such as carbon nanotubes (CNTs) and gold nanoparticles (AuNPs), which have excellent plasmonic properties,

offer numerous possibilities for accurate sensor design and multi-analyte detection 
capabilities. Carbon nanotubes have become the focus of intense investigation since their discovery, as they can promote the electrochemical reactivity of important biomolecules and enhance electron-transfer reactions. In parallel, AuNPs have important applications in numerous areas, including the design of colorimetric sensors. Colorimetric sensors have many advantages for on-site and point-of-care (POC) detection because they can minimize, or even eliminate, the necessity for analytical instruments and can even enable naked-eye observation of assay readouts. The AuNPs are also readily compatible with surface modification with various entities that can aid in the detection process, such as DNA aptamers. DNA aptamers have emerged as a promising alternative to antibodies, as they can deliver high affinity and specificity for proteins, small-molecules, and other targets, but also have the important benefit of being chemically synthesized rather than produced in vivo. Aptamers and AuNPs have already been combined in a number of sensor formats. ${ }^{138}$

Low cost and portability are important characteristics for on-site detection, especially in settings where time is of the essence, such as medical emergencies. Paper-based sensors are especially well-suited for such applications, as they enable the fabrication of simple, cheap, portable, and disposable devices. The adaptation of electrochemical sensing systems into paper-based devices could, therefore, facilitate access to simple, low-cost, on-site detection assays. For example, CNTs and AuNPs can be combined by using a simple fabrication strategy — such as vacuum filtration (VF), which requires only basic lab equipment - to produce a hybrid material with high surface area that is highly useful for electrochemical analyte detection, and which can readily be formed into electrodes of desired size and shape on a paper substrate. 


\subsection{Motivation and goal}

To date, researchers have primarily focused on the fabrication of paper-based sensors for use in developing world diagnostic applications, where simplicity and ease-of-use are highly desirable. ${ }^{61}$ The goal of my project is to design platforms with simple fabrication strategies involving novel nanomaterials, and to pair them with energy-efficient transduction systems such as electrochemical and colorimetric sensors as a practical tool for on-site diagnostics and drug detection. We have developed a platform for the fast and reproducible manufacture of disposable, paper-based, conductive thin gold films, in which we utilize an efficient VF technique to deposit an underlayer of arbitrarily organized, electrochemically inert, metallic single-walled carbon nanotubes (MSWCNTs) onto a mixed cellulose ester (MCE) filter paper. The underlayer, in turn, serves as a rigid matrix for forming an upper layer of AuNPs, as described in Chapter 3. The method produces highly conductive porous gold films under ambient conditions, with no post-growth, cleaning or sintering steps, which offer excellent detection capabilities and can distinguish the presence of dopamine (DA) and serotonin (5-HT) either individually or simultaneously - with a resolution greatly exceeding that of commercially-available flat gold slides or porous gold films prepared by more laborintensive electrodeposition techniques. Our hybrid film is designed to meet the requirements for assembling a cheap and portable device that also offers the capacity for detection with high sensitivity and selectivity, and multi-analyte discrimination. There are several methods by which CNTs can be chemically modified to tune their characteristics for a range of applications. However such modifications will ramp up the production cost, which runs counter to our objective in my work. We have therefore used unmodified 
PureTubes single-walled carbon nanotubes (P-SWCNTs). After using our VF technique to form a thin film of P-SWCNTs on MCE paper, we developed a sensor device for the electrochemical detection of beta-nicotinamide adenine dinucleotide disodium salt hydrate (NADH) in biosamples and other complex matrices, as described in Chapter 4. To further expand our goal of developing a detection platform that can eliminate the use of instrumentation, we propose a colorimetric detection strategy using AuNPs, DNA aptamers, and the exonuclease III (Exo III) enzyme for on-site cocaine detection. This enzyme-based amplification is an extremely straightforward detection method, as the change in color can readily be visualized by eye. We employ an enzyme-assisted target recycling (EATR) strategy, in which we have optimized the probe density to ensure sufficient hybridization efficiency with a rapid detection time and excellent specificity for cocaine, as described in Chapter 5.

\subsection{Scope of the dissertation}

Chapter 2 of the present thesis provides a broad literature review and background on the nanomaterials and methods used in the construction of our electrochemical biosensor. It also describes colorimetric sensors developed for cocaine detection and offers an introduction to carbon nanotubes, and the synthesis of AuNPs and their application in electrochemical and colorimetric detection platforms. This chapter also reviews current thin gold film fabrication strategies, along with materials and methods used for the detection of targets such as NADH. Finally, this chapter provides an introduction to the use of DNA aptamers for cocaine detection, and their use in conjunction with AuNPs for analyte detection. Chapter 3 details the fabrication of a paper-based porous gold film using our VF technique for electrochemical detection of DA and 5-HT. Chapter 4 focuses 
on the application of VF fabricated P-SWCNT films for the electrochemical detection of $\mathrm{NADH}$ in complex biosamples. Chapter 5 examines the evolution of cocaine aptamer design to increase their specificity as well as the rate of the enzyme reaction required to generate a visible color change from the aggregation of AuNPs in our colorimetric sensor. Finally, Chapter 6 provides a summary of the dissertation and outlines a plan for further research. 


\section{CHAPTER 2: Background and literature review}

\subsection{Biosensors}

The demand for sensitive monitoring technologies capable of rapid and accurate analysis is steadily growing across a range of fields, including disease diagnostics, ${ }^{1}$ forensics, ${ }^{2}$ environmental protection, ${ }^{3}$ drug development, ${ }^{4}$ and hygiene. ${ }^{5}$ Among the most promising areas of development are biosensors, devices that contain biological elements capable of recognizing specific target molecules, where target binding is subsequently transduced into a semi-quantitative or quantitative signal that is roughly proportional to the target concentration (Figure 1). The field was born in 1962, when Leland Clark, Jr.- known today as 'the father of biosensors' - described a system for glucose detection that used a dialysis membrane to trap the enzyme glucose oxidase (GOX) against a platinum electrode. $^{6}$

The affectability of a given biosensing platform is defined by its capacity to transduce the target binding process into a perceptible signal, while the selectivity is determined by the biosensor's binding affinity and specificity for its target.

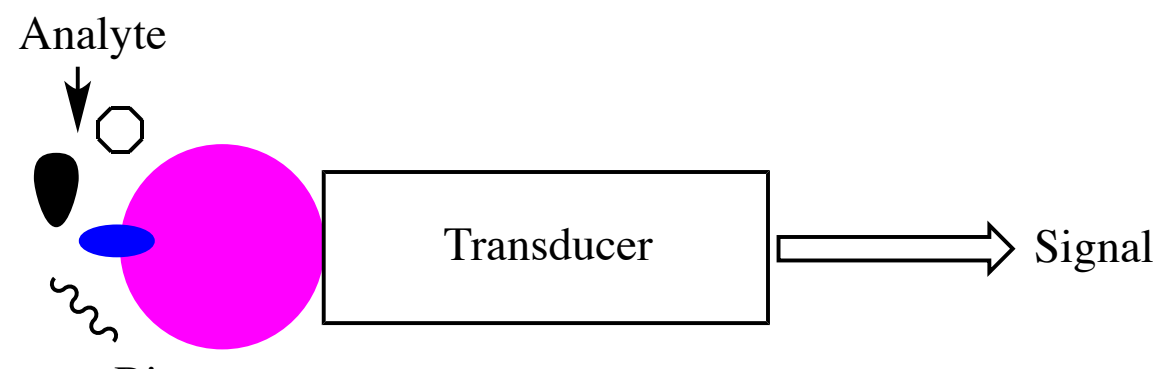

Bio-receptor

Figure 1. The working principle of a biosensor.

A variety of materials are now being investigated to assess their performance in different detection frameworks. 


\subsection{Nanomaterials}

Steady progress in nanotechnology has led to the development of improved nanomaterials that can perform different functions such as imaging, detecting, and targeting. By modulating their surface area, size, shape and configuration, it becomes possible to optimize their chemical and physical properties for use in the creation of biosensors with tremendous sensitivity.

\subsubsection{Carbon nanotubes}

Carbon nanotubes (CNTs) are one of the widely investigated nanomaterials of the, $21^{\text {st }}$ century, and their extraordinary characteristics have been employed in diverse research areas. Since 2006, worldwide CNT production capacity has multiplied at least 10-fold, and the annual CNT-related journal publications and patents continue to grow steadily. Carbon nanomaterials exhibit considerable structural diversity, existing in allotropic forms such as nanodiamond, graphene, amorphous carbon, Fullerene C60, and singlewalled nanotubes (SWNTs).

To date, CNTs, graphene, and their related species are most widely used for detecting strategies of analytes. Carbon nanotubes are tubular rolled sheets of graphene, and include both single-walled (SWNTs) and multi-walled (MWNTs) species. ${ }^{7,8}$ The SWNTs exhibit metallic, semi-metallic and semiconducting properties that can be exploited for detecting applications. ${ }^{9}$ Chemical vapor deposition (CVD) is the standard means for highvolume CNT production, and generally, uses fluidized bed reactors that promote gas diffusion and heat transfer to metal catalyst nanoparticles. ${ }^{8,}{ }^{10}$ Carbon nanotubes have large activated surfaces and oxygen-containing activated sites that are ideal for performing electrocatalytic oxidation at low potentials. ${ }^{11}$ A number of methods have been 
established to functionalize CNT sidewalls for the purpose of expanding their potential applications, but their hydrophobic nature and insolubility in many solvents hinders their use. $^{12}$

\subsubsection{Gold nanoparticles}

Colloidal metal nanoparticles have shown great potential in optical, electronic, and magnetic applications, and nanoparticle-based electroanalytical techniques are especially promising for the construction of advanced chemical- and biosensor-based detection platforms. ${ }^{13}$ Biocompatibility, chemical stability, and oxidation resistance are some of the salient characteristics of gold nanoparticles (AuNPs) that make them adaptable for applications such as chemical sensing, ${ }^{14}$ catalysis, ${ }^{15}$ electronics,${ }^{16}$ imaging, ${ }^{17}$ and biological labeling. ${ }^{18}$ Colloidal AuNPs also exhibit high surface area, ${ }^{19}$ good surface chemistry for chemical modifications and high electrical conductivity, ${ }^{20}$ enabling them to effectively catalyze the redox process for molecules of analytical interest, leading to lower detection limits. The electrical conductivity, catalytic activity, and optical properties of these nanoparticles is largely dependent on their shape and size. ${ }^{21}$ Electronic absorption spectroscopy provides information regarding the UV/Vis region, which is dominated by transitions involving the transfer of electrons from ligand orbitals to metal upon absorption of a photon. ${ }^{22}$ Monodispersed AuNPs with a diameter of $\sim 30 \mathrm{~nm}$ will exhibit concerted oscillations (surface plasmons) of electron charge by the free electrons of the colloidal nanoparticle due to the propagation of the light ray, such that the

oscillations are in resonance with the frequency of the light. ${ }^{22}$ The observed surface plasmon absorption phenomenon results in absorption of light in the blue-green portion 
of the spectrum $(\sim 450 \mathrm{~nm})$, while the red light is reflected. Further increases in particle size result in greater absorption of red light and reflection of blue or purple light. ${ }^{22}$

\subsubsection{Mechanisms of AuNP synthesis}

Citrate reduction (also known as the Turkevich method) is one of the most widely used methods for AuNP synthesis because of its simplicity, energy efficiency, inexpensive reagents, and the tunable size range of the resulting particles. ${ }^{23}$ The process, which yields colloidal AuNPs typically ranging in diameter from 10-100 nm, entails the reduction of total ionic $\mathrm{Au}^{+3}$ to atomic $\mathrm{Au}^{0}$ by citrate anions. This chemical reduction results in nucleation and successive growth in situ to produce spherical or quasi-spherical AuNPs. The first step is the oxidation of sodium citrate, yielding dicarboxy acetone or sodium acetone dicarboxylate (SADC), followed by reduction of the auric salt to aurous salt and then $\mathrm{Au}^{0}{ }^{24}$ Depending on the $\mathrm{pH}$ of the reaction solution, $\mathrm{AuCl}_{4}^{-}$ions $(\mathrm{pH} 3.3$ ) are hydrolyzed into different types of auric precursor ions: $\mathrm{AuCl}_{3}(\mathrm{OH})^{-}\left(\mathrm{pH}\right.$ 6.2), $\mathrm{AuCl}_{2}$ $(\mathrm{OH})_{2}^{-}\left(\mathrm{pH}\right.$ 7.1), $\mathrm{AuCl}(\mathrm{OH})_{3}{ }^{-}\left(\mathrm{pH}\right.$ 8.1), and $\mathrm{Au}(\mathrm{OH})_{4}{ }^{-}\left(\mathrm{pH}\right.$ 12.9). ${ }^{24}$ Their reactivity decreases in the following sequence: $\mathrm{AuCl}_{4}{ }^{-}>\mathrm{AuCl}_{3}(\mathrm{OH})^{-}>\mathrm{AuCl}_{2}(\mathrm{OH})_{2}{ }^{-}>\mathrm{AuCl}$ $(\mathrm{OH})_{3}{ }^{-}>\mathrm{Au}(\mathrm{OH})_{4}{ }^{-24}$ To minimize the $\mathrm{pH}$ buffer effect of citrate, pre-mixed $\mathrm{HAuCl}_{4}$ citrate can be rapidly added to the boiling mixture. The presence of just a single reactive ion species, such as $\mathrm{AuCl}_{3}(\mathrm{OH})^{-}$, can facilitate homogenous nucleation and is more likely to result in monodispersion than mixtures containing multiple precursor ions. ${ }^{25}$

When the $\mathrm{pH}$ of the reaction solution is between 3.3 and 6.2 the solution is monodisperse. Rapid formation of SADC favors particles with a narrow size distribution, but increased $\mathrm{pH}$ and temperature, SADC decomposes to acetone, which reduces the auric precursor to $\mathrm{AuCl}^{24,25}$ This results in secondary nucleation, broadening the size distribution of the AuNPs. Forming macromolecular $\mathrm{SADC} / \mathrm{AuCl}$ complexes requires a minimum of 2 
SADC to tether $3 \mathrm{AuCl}\left(\mathrm{Au}^{+}\right)$, corresponding to a citrate: $\mathrm{HAuCl}_{4}$ molar ratio of 0.67 . Citrate anions stabilize the surface of AuNPs during particle formation by acting as individual, noninteracting, negatively-charged adsorbed species on the AuNP surface. Steric repulsion by this citrate layer stabilizes the AuNPs. ${ }^{24}$ Citrate anions coordinate to the AuNP surface by inner-sphere complexation with their carboxylate groups, and there are trace amounts of $\mathrm{AuCl}^{-}, \mathrm{Cl}^{-}$, and $\mathrm{OH}^{-}$on the metal surface. The hydroxyl group of citrate forms a 5-membered chelating coordination structure with the metal atom. ${ }^{24}$ However, a few hydroxyl groups on the adsorbed citrate remain free and available for other interactions. Attenuated total reflectance infrared (ATR-IR) spectroscopy studies have revealed the presence of dangling citrate ions that are not in direct contact with the surface of the nanoparticle. The existence of these free carboxylate groups is responsible for the negative charge of the AuNPs, and recent studies indicate that the central carboxylate groups of the dangling citrate anion may be responsible for electrostatic repulsion between AuNPs. ${ }^{26}$ The addition of thiols to the citrate-stabilized AuNPs leads to coadsorption of thiols between the citrate layers. ${ }^{26}$ The presence of non-hydrogenbonded hydroxyl groups of surface citrates may be promoted by the addition of alkanethiols, which results in the removal of weakly-adsorbed hydroxyl carboxylate derivatives from the surface. ${ }^{26}$

\subsubsection{Factors governing AuNP size and dispersion}

The prediction of size and polydispersity is critical for systematic synthesis of AuNPs. High concentrations of gold salt or low concentrations of citrate often lead to aggregation and polydispersion, whereas high concentrations of citrate produce stable, smaller AuNPs. ${ }^{25}$ Both the ratio of gold salt to sodium citrate (in its capacity as a reducing agent) 
and the rate of adsorption of sodium citrate (in its role as a stabilizer) will influence the size of the AuNPs. ${ }^{27}$ The reduction capability of citrate is limited to particles with diameter $>10 \mathrm{~nm}^{25}$ In general, monodispersion is seen in smaller-sized AuNPs, particularly $<20-\mathrm{nm}$ diameter, whereas polydispersion is observed in particles with diameter $>20 \mathrm{~nm}^{25}$

The controlling factors for particle size and polydispersion include precursor concentration, rate of addition of precursors, state of mixing in the reactor, and other variables. ${ }^{24}$ The reaction rate largely depends on the temperature, as higher temperatures shorten the time required to reach the activation energy for the reduction reaction, and rapid coagulation yields more monodisperse spherical particles. ${ }^{24}$ Increased stirring speed at higher temperatures will result in smaller AuNPs, whereas slower stirring results in inhomogeneous mixing and nucleation of reactants, leading to a broad size distribution of AuNPs. ${ }^{24}$ In a modified Turkevich method, Xia et. al added pre-mixed $\mathrm{HAuCl}_{4} /$ citrate to boiling water with trace amounts of $\mathrm{AgNO}_{3}$ in the mixture to achieve narrow size distribution and uniform quasi-spherical shape. ${ }^{28}$ The hydrodynamic size of the particles in solution can be measured using dynamic light scattering (DLS) to analyze the modulation of the intensity of scattered light that passes through the colloidal solution as a function of time. Comparison of DLS data to transmission electron microscopy (TEM) images reveals the aggregation state of the particles. ${ }^{29}$ For non-aggregated particles in suspension, the DLS diameter will be slightly higher than that shown by the TEM by an offset that is a function of the capping material and the solvent layer attached to the particle as it moves under the influence of Brownian motion, as expressed by StokesEinstein equation. ${ }^{29}$ 


\subsection{Point of care diagnostics}

Infectious diseases continue to collect a heavy toll regarding morbidity and mortality, especially in developing countries. Expanding access to timely treatment could prove transformative in combatting these diseases, but many patients in low and middle-income countries lack ready access to clinical centers with the means to provide a timely and accurate diagnosis to guide treatment. These communities would benefit greatly from access to the point of care (POC) tests that can be administered on-site by local medical practitioners. ${ }^{30}$ Historically, there has been relatively little interest in developing POC tests for maladies that are mostly prevalent in developing nations, but the situation is now evolving. Quick, affordable and reliable POC tests that require minimal hardware and negligible preparation are now available for HIV, syphilis and gastrointestinal infections, although much more POC tests for other biological analytes are desperately needed. Low cost and portability are critical for POC diagnostics, particularly for medical emergencies or for the diagnosis of highly virulent infections where time is of the essence. ${ }^{30}$

\subsection{Hybrid metallic thin films}

Metallic films that combine the unique properties of CNTs and AuNPs promise to bring out the best in both nanomaterials. ${ }^{31}$ The key goal of manufacturing CNT/AuNP hybrids is to improve their electrical, synthetic, and mechanical properties in material-based applications. $^{31,32}$ Methods for making such hybrids can be classified in terms of direct or indirect strategies. In direct methods, metal nanoparticles are made in a single-step on the surface of CNTs. Indirect methods rely on two separate steps, in which functionalized CNTs are manufactured first, followed by the synthesis of nanoparticles. Certain properties of the nanotubes could hypothetically be improved using surface treatments 
utilizing silver, gold, platinum, palladium or rhodium for the immobilization of nanoparticles. ${ }^{33-35}$ Composite hybrid materials are created by joining the nanoparticles to the external surface of the CNTs either covalently or non-covalently.

\subsubsection{Gold thin films}

Porous thin gold films have shown promise for applications in catalysis, ${ }^{36}$ biosensors,${ }^{37}$ fuel cells ${ }^{37}$ and supercapacitors ${ }^{37}$ as a result of high surface-to-volume ratio, great inplane conductivity, and upgraded electron exchange rates. ${ }^{37}$ Such films can be manufactured by either layout-coordinated or template-free strategies. Layoutcoordinated techniques ordinarily comprise electrodeposition, ${ }^{38-41}$ filtration, ${ }^{42}$ or thermal evaporation/sputter coating ${ }^{43,44}$ on unbending latex or $\mathrm{SiO}_{2}$ sphere templates, followed by hydrofluoric acid corrosive etching to remove the template. Gold films generated by such means exhibit uniform pore distribution, but post-dissolution of the template is timeconsuming. These strategies tend to deliver limited quantities of nanostructures in a given run, and the electrocatalytic and detecting capabilities of such films are inadequate. ${ }^{45-47}$ Template-free techniques employ either direct ${ }^{48,} 49$ or hydrogen bubble-directed electrodeposition $^{50,51}$ of gold nanostructures onto a conductive surface. Hydrogen bubble-templated electrodeposition has been used to shape porous nickel, copper, tin, silver, palladium, and lead films. ${ }^{50,52}$ However, the development of porous gold films requires a lot of $\mathrm{NH}_{4}{ }^{+}(>0.5 \mathrm{M})$ because of the considerable hydrogen evolution on the film, producing flaky froths that regularly contain substantial numbers of cracks and thus increase the capacitance current. ${ }^{50,52}$ Chemical dealloying is another option for generating porous gold films from gold-silver compounds. By changing the alloy composition and dealloying conditions, one can effortlessly control the pore structure. 
However, it is difficult to efficiently extract the silver in the dealloying process, and trace measures of silver can alter or complicate the interface chemistry. ${ }^{53-55}$

\subsubsection{Paper as substrate}

The adaptation of electrochemical sensing systems into paper-based devices has proven a major boon for accessible diagnostic testing, with several prominent advantages relative to traditional polymer- and glass-based devices. ${ }^{56,57}$ Paper substrates contribute to a biochemical analysis by capillary wicking of aqueous solutions through the cellulose fibers, providing a means of transport for fluids that does not require any external instrumentation or power source. ${ }^{58}$ Paper is lightweight and flexible, simplifying the logistics of transport and storage, and there are simple methods for patterning and processing paper that can be used to form microfluidic channels ${ }^{58}$ Furthermore, the low cost and combustibility of paper make it suitable for disposable and safe single-use tests. $^{59}$

\subsubsection{Paper-based gold thin films}

There is a clear need for a low-cost, simple and environmentally friendly way to quickly and dependably produce thin gold films with high surface-to-volume proportions, great mechanical properties, and excellent conductivity. Paper is adaptable and flexible substrate that offers a promising substrate for conductive gold nanostructure-based films on account of its inert and porous nature and relative abundance. ${ }^{60}$ For example, microfluidic paper-based analytical devices can use the passive capillary-driven flow of aqueous solutions through patterned paper channels to transport sample fluid into distinct detection zones, performing electrochemical detection with the help of an external potentiostat. ${ }^{61}$ The cost of each device is minimal, since gold electrode arrays can be 
mass-produced on paper using various techniques to achieve flexible self-designed patterns and conductive interface platforms with excellent speed and reproducibility. ${ }^{61}$ Velev et al. made permeable gold films on polycarbonate films by utilizing $1-\mu \mathrm{m}$ polystyrene latex microspheres as a thickly-pressed template to decrease the film pore size, and then used filtration to create a 15-25 $\mathrm{nm}$ upper layer of colloidal gold particles. $^{42}$ After removing the latex spheres from the composite, they got conductive gold flakes with a porous but split three-dimensional structure. ${ }^{42}$ Making a gold film on cellulose filter paper by ink-jet printing provides a simple, efficient, and inexpensive alternative for producing robust thin film electronics. To frame a conductive gold film on a kaolin-covered paper substrate with no layout, Määttänen et al. inkjet-printed a layer of dodecanethiol-altered AuNPs and then used infrared sintering to transform these nonconductive particles into a conductive gold film. ${ }^{62-65}$ These films exhibit $\sim 10 \%$ of the conductivity of solid bulk gold and have been used in a variety of different applications. However, the porosity of the paper-based gold film is decreased post-sintering. ${ }^{62-65} \mathrm{Hu}$ et al. created permeable gold terminals on cellulose layers by consolidating inkjet-printed, non-conductive AuNPs with the self-catalytic development of the printed particles to frame a consistent gold leading layer on the paper. ${ }^{66}$ Although these paper-based permeable gold film clusters showed great conductivity and enabled sensitive electrochemical detection of oxygen, their manufacture required numerous creation steps —including seven rounds of inkjet printing and eight cycles of AuNP synthesis. ${ }^{66}$

\subsection{Electrochemical detection of dopamine and serotonin}

The basic principle for electrochemical biosensors is that chemical reactions between immobilized biomolecules and the target analyte produce or consume ions or electrons, 
which affect electrical qualities of the solution such as electric currentor potential. ${ }^{67}$ Excellent reproducibility and accuracy, a wide linear response range, low detection limits, and real-time measurement capabilities are among the key advantages of electrochemical sensors. ${ }^{67}$ The neurotransmitters dopamine (DA) and serotonin (5-HT) are electro-active molecules and hence can be detected electrochemically, and Retna Raj and co-workers fabricated an AuNP-modified gold electrode for the detection of DA, ${ }^{68}$ using a self-assembled monolayer technique to deposit AuNPs on an amine-terminated monolayer of cystamine on a polycrystalline gold electrode. Recently, Xue et al. used a molecular imprinting technique to fabricate a conductive film of AuNP-doped molecular imprinted polymers (AuNP/MIPs) for the detection of trace amounts of DA in human serum. ${ }^{69}$ They introduced AuNPs functionalized with p-aminobenzenethiol (p-ATP) onto modified gold electrodes using an electropolymerization technique. ${ }^{69}$ Shim et al. described an electrochemical biosensor utilizing AuNPs decorated with indium tin oxide (ITO) substrate for highly sensitive detection of DA in human serum and urine. ${ }^{70}$ The augmented oxidation current and lower oxidation potentials achieved with this approach were attributed to the ability of AuNP/ITO electrodes to accelerate the rate of electron transfer and thus increase their electrocatalytic activity. ${ }^{70}$

\subsection{NADH and its biological significance}

Nicotinamide adenine dinucleotide (reduced) is a coenzyme that is ubiquitously found in all living cells, and which plays a prominent role in electron transfer as well as numerous metabolic reactions. ${ }^{71-73}$ Intracellular NADH serves as a biomarker for several physiological and pathological events. ${ }^{74-77}$ Fluctuations in NADH levels in cells and tissues can correspond with intracellular redox status, ${ }^{78}$ apoptosis, ${ }^{78}$ neurodegenerative 
diseases, ${ }^{73,75,76}$ and mitochondrial anomalies associated with cancer. ${ }^{79}$ Nicotinamide adenine dinucleotide (reduced) also stimulates biosynthesis of dopamine and tyrosine hydroxylase, making it an effective candidate in the treatment of Parkinson's syndrome. ${ }^{80}$ Conversion of NADH is also elevated in hyperplasic cancer cells compared to normal cells. ${ }^{79}$ In total, this biomolecule is known to participate in more than 300 enzymatic oxidation/reduction reactions in vivo. ${ }^{71-73}$ Accordingly, tools for sensitive measurement of NADH levels could prove very useful in a variety of diagnostic and prognostic contexts.

\subsubsection{Current methods for detecting NADH in biosamples and cell extracts}

Enzymatic assays are widely used for determining levels of intracellular NADH. One method for estimating free $\mathrm{NADH}$ in rat liver entails measuring concentrations of substrates of $\beta$-hydroxybutyrate and glutamate-dehydrogenase enzymes. ${ }^{81}$ Other enzymatic assays have targeted the role of NADH as an electron carrier in metabolic reactions. ${ }^{82}$ These assays utilize this function of NADH to determine its concentration. Although these assays can quantify the total amount of pyridine dinucleotides in a cell, they cannot directly differentiate between NAD and NADH. For the purpose of differentiation, quantification is usually done in two steps, in which an extraction step is followed by quantification. The process can entail either a separate extraction method, ${ }^{83}$ where NADH is extracted in an alkaline solution to decompose NAD, or a single extraction in which both NAD and NADH are extracted using neutral or mild basic solutions. ${ }^{84}$ Nicotinamide adenine dinucleotide (reduced) was quantified in a mouse neuroblastoma cell line using an enzymatic cycling assay with a separate extraction method involving selective decomposition of NAD. ${ }^{83}$ Bench et al. extracted NADH from a lyophilized Saccharomyces cerevisiae extract using organic solvents, with further 
quantitation achieved using HPLC and UV-Vis absorbance detection. ${ }^{85}$ Free and bound NADH have also been quantitated in complex samples using excited fluorescence spectra. Fluorometric methods exploit the strong fluorescence of NADH $\left(\lambda_{\mathrm{ex}}=340 \mathrm{~nm}\right.$, $\lambda_{\mathrm{em}}=460 \mathrm{~nm}$ ), relative to the minimal fluorescent properties of NAD. ${ }^{86}$ These assays are simple, but are plagued by strong background, and the autofluorescence of NADH is weak in comparison. Physical cell disruption methods such as ultrasonication, highpressure homogenization, high-speed bead milling, and chemical methods such as chemical permeation and enzymatic lysis are generally used for extraction of NADH for analysis. ${ }^{87-89}$ Extracts containing NADH prepared from yeast cells by treatment with snailase $^{90}$ and $\operatorname{SDS}^{91}$ enable quantitation of levels of intracellular NADH when the fluorescence spectra are compared against standard NADH solutions. Indirect fluorescence assays based on principles such as the reduction of resazurin to resafurin by $\mathrm{NADH}$ in the presence of diaphorase ${ }^{92}$ work as an indicator for levels of intracellular $\mathrm{NADH}$, and Amplex Red can also be used for assays based on a similar mechanism. ${ }^{93} \mathrm{~A}$ variety of microscopic techniques have also been developed for quantitation of intracellular NADH, but these are expensive and laborious when used only for this purpose. The limitations of the various assays described above make them ill-suited for routine use in diagnostic, clinical or forensic settings, and there remains a demand for rapid, inexpensive, and disposable sensing platforms.

\subsubsection{In vitro applications of NADH}

A cocaine metabolite assay developed by Syva detects the presence of benzoylecgonine in urine samples by using a competitive immunoassay strategy (EMIT), which causes a change in the reaction kinetics for NADH production depending on the presence or 
absence of the metabolite. ${ }^{94}$ The drug present in the biosample competes with the druglabeled enzyme for a limited number of binding sites on the antibody. ${ }^{94}$ If the concentration of drug in the sample is sufficient to competitively bind to the antibody sites, the enzyme-labeled drug is free in solution to break down glucose-6-phosphate, resulting in the release of a single hydrogen ion $\left(\mathrm{H}^{+}\right)$. In contrast, if the conjugate is bound to the antibody, the enzyme activity is reduced. ${ }^{94}$ Nicotinamide adenine dinucleotide present in the assay reagents reacts with the released hydrogen ion to form $\mathrm{NADH}$, and the amount of NADH produced during the EMIT reaction is directly proportional to the amount of drug present in the sample, and can generally be detected using ultraviolet spectrophotometry. ${ }^{94}$ However, spectroscopic methods possess an inherent disadvantage when working with complex samples due to spectral interference. Furthermore, although this assay uses NADH as a proxy for detecting other targets, it is not intended for detecting naturally occurring NADH concentrations in vivo.

\subsubsection{Electrochemical detection of NADH in complex samples}

Biosensors are still rarely used because of their unpredictable behavior in 'real-world' samples, despite strong performance with laboratory standards under experimental conditions. Researchers are therefore working to optimize existing platforms in order to improve their applicability in complex biosamples for routine use as commercial assays. Electrochemical methods have advanced considerably as a tool for detecting many important biological analytes, thanks to the impressive miniaturization of modern microelectronics and the relative paucity of electroactive contaminants in most biological samples. ${ }^{95}$ Nicotinamide adenine dinucleotide (reduced)-associated enzymatic reactions are of particular interest in this space; NADH is electrochemically active, and 
conventional solid electrodes composed of gold, ${ }^{96}$ platinum $^{97}$, and glassy carbon ${ }^{98}$ have been used for NADH oxidation. However, NADH can only be oxidized on these electrodes at potentials above $+1.0 \mathrm{~V}$. Consequently, researchers have employed various electron mediators such as organic dyes, conducting polymers and thio-substituted nucleobases to facilitate NADH oxidation. Although electron mediators appear to offer a promising potential solution, the utility of these assays is limited by defects in surface modification, and the adsorption of $\mathrm{NADH}$ and $\mathrm{NAD}^{+}$to the electrode also leads to surface fouling, resulting in poor sensitivity, stability, and reproducibility. ${ }^{96-98}$ Jenkins et al. developed an alternative electrochemical method for quantifying NADH in whole blood using flow-injection analysis. In their approach, NADH produced by dehydrogenase is oxidized by an electron-transfer coupling reagent, 2,6dichloroindophenol (DCIP), and the reduced form of DCIP $\left(\mathrm{DCIPH}_{2}\right)$ is measured via amperometry. ${ }^{99}$ Bala et al. developed an AuNP/reduced graphene oxide (GO) composite sensing platform, which was formed directly on a GCE via in situ electrochemical reduction of GO and $\mathrm{Au}^{3+}$ to detect NADH spiked into urine samples. ${ }^{100}$ The electrode surface provides many favorable sites for electron transfer to biomolecules, and would be helpful for accelerating electron transfer between the electrode and species in solution. ${ }^{100}$ However, the number of steps involved in fabricating such electrodes is time-consuming. Therefore, it remains essential to find an efficient strategy for electrode fabrication that also accelerates the process of interfacial electron transfer between NADH and the electrode surface.

\subsubsection{CNT-based electrochemical detection of NADH}

The distinctive structural and electrical properties of CNTs have aroused considerable 
interest as a tool for electrochemical detection. Carbon nanotubes have large activated surfaces and edge-plane sites that are ideal for performing NADH oxidation at low potentials without the help of any redox mediators, minimizing surface fouling. ${ }^{101}$ Borondoped carbon nanotube (BCNT) modified electrodes have been investigated for the detection of NADH, in which a polished GCE was used as a substrate onto which a suspension of BCNT was dropped and dried under an infrared lamp. ${ }^{102}$ The BCNTs exhibited faster electron-transfer rates as a result of the higher proportion of edge-plane sites and more oxygen-rich groups presented on the BCNT surface. ${ }^{102}$ Another report describes composite CNT-polymer electrodes, where poly-(3-methylthiophene) multiwalled CNT hybrids were prepared on GCEs that were immersed into a monomeric 3methylthiophene (3MT) solution in acetonitrile and sodium perchlorate for electropolymerization. ${ }^{103}$ However, these electrodes suffered from poor selectivity. Most CNT films are prepared by solution-deposition methods, in which CNTs are dispersed in solvent (e.g., DMF, sulfuric acid or chitosan) and cast onto the electrode surface, and then dried in air or vacuum. ${ }^{104}$ However, CNT films made by this method are not stable, and are limited by high background and weak signal. Self-assembly methods overcome some of these drawbacks. ${ }^{105}$ The CNTs have been self-assembled onto electrode surfaces by electrostatic attraction, hydrophobic interactions or covalent bond. Most substrates used in this method are solid electrodes, such as gold electrodes or GCEs. ${ }^{105}$ Substrates and CNTs need additional modifications for self-assembly, which makes this method costly, complicated, and time-consuming. Recently, SWCNT-based "Bucky-paper" was used as a new substrate for NADH oxidation. ${ }^{106}$ However, it suffers from very high background due to the thickness of the paper $(\sim 350 \mu \mathrm{m})$, and the SWCNTs must be 
modified with an electrocatalyst for NADH oxidation. The SWCNT films have also been used for electrochemical sensing without surface activation/functionalization. Several groups have explored paper-based substrates as rapid, inexpensive, and disposable sensing platforms for use in industrial and clinical settings. Eichhorn et al. examined the growth of CNTs on the surface of carbonized cellulose fibers. ${ }^{107}$ Using this approach, the overpotential of NADH decreased from over 0.8 to $0.6 \mathrm{~V}$ for the CNT-modified carbon fiber electrode, indicating that the presence of CNTs could lower the potential of NADH oxidation and thereby reduce the likelihood of electrode fouling. ${ }^{107}$ Our current research is focused on combining the electrocatalytic advantages of CNTs with inexpensive, disposable cellulose-based substrates, by fabricating thin CNT films on MCE filter paper with a simple VF technique. Many groups have previously reported such methods for fabricating thin, unmodified CNT films on paper, producing a homogenous porous film. However, these films have rarely been applied in the context of detection in biosamples.

\subsection{Cocaine abuse}

Cocaine is a central nervous system (CNS) stimulant derived from the leaves of the coca plant that has two major pharmacological actions. ${ }^{108}$ It function as both a local anesthetic and an indirectly-acting sympathomimetic, with properties similar to amphetamines. ${ }^{108}$ It is classified as a Schedule II drug, and is one of the main causes of emergency hospital visits and illicit drug-related deaths in the United States. ${ }^{108}$ According to the 2014 National Survey on Drug Use and Health (NSDUH), roughly 1.5 million people aged 12 or older have used cocaine. ${ }^{109}$ Cocaine binds differentially to the dopamine, serotonin, and norepinephrine transport proteins, and directly inhibits the re-uptake of these neurotransmitters into pre-synaptic neurons, resulting in euphoria, pleasure and 
addiction. ${ }^{110,111}$ Prolonged abuse of cocaine may cause increased heart rate, high blood pressure, irritability, anxiety, paranoia and hallucinations as well as other symptoms. ${ }^{111}$ Overdoses may lead to coma or even death, and may cause birth defects and premature delivery in pregnant women. ${ }^{110}$

\subsubsection{Current methods for presumptive screening of cocaine}

Over the years, many techniques - both qualitative and quantitative-have been employed for cocaine detection, with varying sensitivity and specificity for this drug or its metabolites. Traditionally, detection is first achieved with a relatively rapid and lowcost presumptive screening test, where a positive result is followed by a more sensitive and accurate, but also costly and labor-intensive, confirmatory test. Gas chromatography/mass spectrometry (GC/MS) is the most sensitive, reliable and extremely specific technique available today for the detection of cocaine, and is considered the gold standard for confirmatory testing in this regard. ${ }^{112-118}$ Immunoassays are the most common method for initial presumptive screening. Among the most popular commercially available tests are enzyme-linked immunosorbent assays (ELISA), ${ }^{119,} 120$ EMIT, ${ }^{112}$ fluorescence polarization immunoassays $(\text { FPIA })^{121}$ and radioimmunoassays $(\mathrm{RIA})^{113}$ for the quantitative and qualitative detection of cocaine and its major metabolites in biofluids. Most early immunoassays used ELISA, but these tests are timeconsuming and expensive. The EMIT testing is more commonly used at the moment and is the least expensive assay for simple, rapid screening, but is also plagued by low sensitivity. The RIA offers sensitive detection, but its expensive reagents (including the need for radioactive materials) and the technical skill required to perform the assay make it a less popular choice. In general, antibody-based screening tests suffer from the major 
drawback of not being able to distinguish between drugs of abuse and structurally similar, legal analogs, resulting in false positives. ${ }^{115}$ Therefore, there remains a need for assays that enable rapid, low-cost, sensitive and accurate on-site drug screening.

\subsubsection{Aptamers as biosensor recognition elements}

There is growing interest in the development of biosensors that make use of aptamers as an alternative to antibodies for recognition elements. ${ }^{122}$ Aptamers are short singlestranded DNA or RNA molecules that bind to various targets with high affinity and specificity, which are typically generated through a process called systemic evolution of ligands by exponential enrichment (SELEX). ${ }^{123}$ In SELEX, an oligonucleotide library is combined with the target of interest, resulting in separation of sequences with binding affinity for that target molecule. These selected sequences are further amplified by polymerase chain reaction (PCR), and the products are subjected to another round of selection. ${ }^{123}$ The selection cycles proceed until one has isolated a pool containing DNA/RNA species with high affinity for the target. ${ }^{123}$ Since their discovery in the 1990 s, numerous aptamers have been selected for various targets, including metal ions, biomolecules, organic molecules, or even whole microorganisms/cells. Aptamers possess numerous advantages for fabrication of biosensors relative to antibodies, including costeffective production, batch-to-batch reproducibility, high binding affinities for their targets and ease of modification for purposes of signal transduction and detection. ${ }^{124-133}$ Aptamers are also not constrained by the immunogenicity issues that can confound antibody generation for certain antigens, and exhibit higher stability against degradation and denaturation. ${ }^{134}$ One can also design SELEX procedures that favor the isolation of aptamers that undergo a target-specific, binding-induced conformational change, and 
these can readily be conjugated to a diverse array of substrates to produce biosensors with high sensitivity and specificity, and which produce minimal background signal in the absence of their target. ${ }^{124-133}$ For example, Soh et al. reported structure-switching aptamers (SSAs) that can act as biosensors without additional modification, optimization, or the use of DNA helper strands. ${ }^{135}$ The target-binding induced conformational change in SSAs is far greater than in conventional aptamers, and the major structural difference between the bound and unbound state enables the use of SSAs in a variety of detection methods. ${ }^{135}$ Recent work from Ebrahimi et al. describes the first successful in vitro selection for methamphetamine with a $\mathrm{K}_{\mathrm{d}}$ in the nanomolar range. ${ }^{136}$

\subsubsection{Colorimetric AuNP-conjugated aptamer sensors}

Ramezani et al. reported an AuNP-based colorimetric triple-helix molecular switch (THMS) system for the detection of tetracycline. Their THMS used target-induced release of a single-stranded DNA signal transduction probe (STP) from the aptamer. ${ }^{137}$ In the absence of tetracycline, the THMS remains stably conjugated with the STP, and AuNPs present in the solution undergo salt-induced aggregation that produces a visible blue readout color. The presence of tetracycline induces a conformational change in the THMS, leading to the release of the STP; the released STP (which is negatively-charged) subsequently adsorbs onto the surface of the AuNPs (which are positively-charged). The presence of STPs on the AuNP surface prevents salt-induced aggregation, and the AuNP solution therefore produces a red color. ${ }^{137}$ Such colorimetric sensors have considerable advantages for on-site and point-of-care detection, because they can minimize or even eliminate the need for analytical instruments and may even allow naked-eye observation. 


\subsubsection{Aptamer-based biosensors for cocaine detection}

The Stojanovic group isolated an aptamer called MNS-4.1, which was the first aptamer to exhibit high specificity and selectivity towards cocaine relative to its major metabolites. ${ }^{132}$ Stojanovic et al. developed a sensor design that employed a fluorophorequencher-modified cocaine-binding aptamer based on MNS 4.1, where the aptamer remains unfolded and produces a fluorescent signal in the absence of cocaine. ${ }^{132}$ The presence of cocaine leads to the formation of a non-canonical three-way junction with a hydrophobic pocket in the aptamer, bringing the fluorophore and quencher into close proximity and causing $\sim 50 \%$ quenching of the fluorescence signal. ${ }^{132}$ They described a limit of detection (LOD) of $10 \mu \mathrm{M}$ cocaine in serum samples using this sensor design. ${ }^{132}$ Cocaine-binding aptamers have been coupled with a variety of transduction methodologies, including colorimetry, ${ }^{138}$ chemiluminescence, ${ }^{139}$ electrochemistry, ${ }^{140}$ and surface-enhanced Raman scattering. ${ }^{141}$ Stojanovic's group also reported a colorimetric sensing platform, in which a $\mathrm{Cy}-7$ dye molecule is displaced from the aptamer's threeway junction in the presence of cocaine, generating a visible colorimetric signal. ${ }^{142}$ However, such assays require the identification of specific dyes that can not only bind to the aptamer efficiently but which are also subsequently displaced by target binding. Although MNS-4.1 preferentially undergoes a conformational change in the presence of cocaine, the unbound aptamer also exists in equilibrium between folded and the unfolded states, which leads to higher background signal and limits the sensitivity of the aptamer. To address this issue, MNS-4.1 was subsequently split into two ${ }^{131}$ or three ${ }^{143,144}$ smaller aptamer fragments that remain separated in the absence of cocaine but assemble in the presence of cocaine, thereby reducing the background. Stojanovic's group used this split 
aptamer for fluorescence detection of cocaine, with the two fragments tagged with a fluorophore and quencher respectively. ${ }^{131}$ Target-induced assembly in the presence of cocaine brought together both fragments, resulting in decreased fluorescence intensity. ${ }^{131}$ However, the splitting of the aptamers had the drawback of reducing target affinity to a $K_{d}$ of $\sim 200 \mu \mathrm{M}$. yielding a poor LOD. ${ }^{131}$ This split aptamer strategy was further modified by the Plaxco group, which developed an electrochemical sensor in which one of the unmodified fragments was attached to a polished gold electrode surface, whereas the other was modified with a redox label (methylene blue) and remained free in solution. ${ }^{140}$ The assembly of aptamer-cocaine tertiary structures upon addition of cocaine brought the redox label into proximity with the electrode surface, generating a large faradaic current. ${ }^{140}$ Willner's group developed an exonuclease III (Exo III)-based aptamer sensor for the amplified detection of cocaine, using a split aptamer consisting of an unmodified fragment and a fluorophore/quencher-modified fragment. ${ }^{145}$ These fragments remain single-stranded in the absence of cocaine, preventing Exo III digestion and thus resulting in quenched fluorescence. The presence of cocaine triggers the assembly of these two fragments into a duplex that can be recognized and digested by Exo III, resulting in release of the quencher and producing a strong fluorescence signal. ${ }^{145}$ The target and unmodified fragment are then released, enabling recycling and signal amplification that results in a higher LOD of $50 \mathrm{nM} .^{145}$ One group reported the colorimetric detection of cocaine based on the catalytic activity of the peroxidase-mimicking G-quadruplex DNAzyme, where one aptamer fragment was modified onto amine-functionalized magnetic nanoparticles while the other remained free in solution. ${ }^{146}$ Cocaine triggered the assembly of the two fragments on the nanoparticle, forming a three-way junction on its 
surface. The G-quadruplex DNAzyme further catalyzed a TMB- $\mathrm{H}_{2} \mathrm{O}_{2}$ reaction, generating a visible color change with a LOD of $50 \mathrm{nM}{ }^{146}$ The AuNPs are one of the most commonly-used nanomaterials for the construction of aptamer-based biosensors, including electrochemical, fluorometric, surface-enhanced Raman scattering, colorimetric, and chemiluminescence imaging arrays. Fan et al. developed a simple colorimetric assay for cocaine detection using unmodified AuNPs and split aptamer fragments in solution. ${ }^{147}$ In the absence of cocaine, the AuNPs remained red because of non-specific adsorption of the aptamer fragments onto the AuNP, while the presence of cocaine led to the formation of aptamer-cocaine ternary structures prevented this adsorption from occurring, resulting in AuNP aggregation and generating a blue color. ${ }^{147}$ A LOD of $2 \mu \mathrm{M}$ for cocaine was achieved with this assay, but higher concentrations of cocaine induced the aggregation of unmodified AuNPs. ${ }^{147}$ Another assay exploited cocaine-induced disassembly of aptamer-linked AuNP aggregates, where a targetinduced conformational change in the presence of cocaine triggers dissociation from the AuNP surface and subsequent disruption of AuNP aggregates. ${ }^{148}$

\subsubsection{Cooperative binding split aptamers}

Cooperative binding, which is defined as the increase in binding affinity observed due to binding at secondary sites, is commonly observed in ligand-binding proteins with multiple binding sites that are sensitive to ligand concentration, such as hemoglobin, ion channels and transcription factors. ${ }^{149-151}$ Breaker et al. in 2004 first noticed the cooperative behavior in some tandem riboswitches, where ligand concentrations were manipulated to control gene expression. ${ }^{152,153}$ Different research groups further adapted this phenomenon into artificial systems, such as molecular beacons, ribozymes, and DNA 
aptamers. Plaxco et al. tried to emulate this cooperative binding by introducing disorder into the parent aptamer to establish a ligand concentration-dependent "switch-like" response. ${ }^{154,155}$ However, this proved ill-suited for a practical sensor platform. More recently, Xiao et al. introduced tandem target-binding domains into a split aptamer, which they termed a cooperative-binding split aptamer (CBSA), for fluorescence-based cocaine detection in complex samples. ${ }^{156}$ The initial cocaine-binding event stabilizes the structure of the split aptamer and aids in subsequent target binding at the second binding domain. The use of a fluorophore/quencher pair in this detection platform results in a sensitive LOD of $50 \mathrm{nM}$ cocaine in $10 \%$ saliva within $15 \mathrm{~min},{ }^{156}$ although a fluorescence spectrophotometer is required to read the output. 


\section{CHAPTER 3: Ambient Filtration Method to Rapidly Prepare Highly Conductive, Paper-Based Porous Gold Films for Electrochemical Biosening}

\subsection{Introduction}

Metallic thin films are basically utilized as a part of photonic ${ }^{157}$ and electronic gadgets. ${ }^{157}$ Specifically, porous thin gold films have picked up extensive consideration in territories including catalysis, ${ }^{158}$ biosensors, ${ }^{159}$ fuel cells ${ }^{160}$ and supercapacitors ${ }^{161}$ due to their unique properties, which incorporate high surface-to-volume ratio, ${ }^{162}$ great in-plane conductivity, ${ }^{163}$ and upgraded electron exchange rates. ${ }^{164}$ Such films have been manufactured by both layout coordinated ${ }^{38-44}$ and template-free $e^{48-51}$ strategies. Layout coordinated techniques ordinarily comprise of electrodeposition, ${ }^{38-41}$ filtration, ${ }^{42}$ or thermal evaporation/sputter coating ${ }^{43,44}$ on unbending latex or $\mathrm{SiO}_{2}$ sphere templates took after by a hydrofluoric corrosive etching venture to evacuate the layouts. The gold films arranged by these rigid format coordinated strategies accomplish uniform pore distribution, however, post-dissolution of the template is tedious. These strategies tend to deliver just constrained amounts of nanostructures in every run, and the electrocatalytic and detecting capabilities of such films is inadequate. ${ }^{45-47}$ Template-free techniques incorporate direct, ${ }^{48,49}$ or hydrogen bubble-directed electrodeposition ${ }^{50,51}$ of gold nanostructures onto a conductive surface. Hydrogen bubble-templated electrodeposition specifically has been generally used to shape porous nickel, copper, tin, silver, palladium, and lead films; be that as it may, the development of porous gold foam requires a lot of $\mathrm{NH}_{4}^{+}(>0.5 \mathrm{M})$ because of large amounts of hydrogen evolution on the film, bringing about flaky froths that regularly contain substantial number of cracks therefore increasing the capacitance current. ${ }^{50,52}$ Chemical dealloying ${ }^{53,54}$ is another famous option to prepare 
porous gold films from gold-silver compounds. Changing the alloy composition and dealloying conditions can effortlessly control the pore structure ${ }^{53-55}$ However, it is difficult to expel the greater part of the silver amid the dealloying process, and trace amount of silver can alter or complicate the interface chemistry. ${ }^{55}$

There is an urgent need for a basic, conservative, and environmentally friendly way to prepare quickly thin gold films with high surface-range to-volume proportions, great mechanical properties, and phenomenal conductivity. Adaptable and flexible substrates, for example, paper have been restored for the improvement of conductive gold nanostructure-based films on account of their inert and porous feature, relative abundance, and practical production. ${ }^{165,62-66}$ Velev et al. fabricated permeable gold films on polycarbonate matrix by utilizing $1 \mu \mathrm{m}$ polystyrene latex microspheres as a thickly pressed layer to decrease the film pore size, and after that utilized filtration to create a 15-25 nm upper layer of colloidal gold particles. ${ }^{42}$ In the wake of expelling the latex spheres from the composite, they obtained conductive brown/gold flakes with a porous however split three-dimensional structure. To frame a conductive gold film on color (kaolin) covered paper substrate with no layout, Määttänen et al. inkjet-printed one layer of dodecanethiol-altered gold nanoparticles (AuNPs) and converted these nonconductive particles into a conductive gold film with infrared (IR) sintering. ${ }^{62-65}$ These created paperbased AuNP terminals have $\sim 10 \%$ of the conductivity of solid bulk gold and have been used for different applications. ${ }^{62-65}$ However, the paper-based gold film decreased its porosity post IR-sintering. Hu et al. created permeable gold film prepared on cellulose layers by consolidating inkjet-printed, nonconductive AuNPs with self-catalytic development of the printed individual particles to frame a conductive gold layer on the 
paper. Although these paper-based permeable gold films showed great conductivity and, sensitive electrochemical detection of oxygen, numerous preparation steps, including seven rounds of inkjet printing and eight cycles of AuNP growth, were required for their manufacture. ${ }^{66}$

In this chapter, we reported a simple strategy for the fast and reproducible manufacture of disposable, paper-based, conductive thin gold films, in which we utilize routine vacuum filtration (VF) technique to deposit an underlayer of arbitrarily adjusted, electrochemically inert, metallic single-walled carbon nanotubes (M-SWCNTs) onto a mixed cellulose ester (MCE) filter paper, which serves as a rigid matrix for forming a top layer of AuNPs. The readiness of these "VF-fabricated gold films" includes insignificant hardware, work, and reagents. The whole procedure takes just 20 min and requires just an essential vacuum-filtration contraption with only two aqueous solutions: Triton X-100dispersed M-SWCNTs and citrate-coated AuNPs. Since the rigid M-SWCNT underlay decreases the pore size of fiter paper and makes a hydrophobic, interconnected system where upon deposit the AuNPs, a permeable thin gold film (thickness $\sim 40 \mathrm{~nm}$ ) can be prepared rapidly, without the requirement for thermal annealing, postgrowth, or sintering steps. The subsequent gold films demonstrated great conductivity, large surface area, and excellent electrocatalytic signal to electroactive biomolecules. We found that the porous elements of the VF-fabricated gold films allow simultaneous detection of two analytes in a mixture with determination that enormously surpasses either bought gold slides or electrodeposited, paper-based gold films. This indicates the potential for utilizing such films as a part of expendable sensors for the identification of numerous analytes. The size of these paper-based gold electrodes can be accurately controlled, and the subsequent 
paper-based gold films could be post-transferred onto any wanted substrate without harming their structure or integrity.

\subsection{Materials and methods}

3.2.1 Chemicals: Gold(III) chloride trihydrate, trisodium citrate dihydrate, Triton X-100, dopamine hydrochloride, serotonin hydrochloride, phosphate-buffered saline (10X PBS, pH 7.4), sulfuric acid (95-98\%), nitric acid (70\%), hydrochloric acid (36.5-38\%), hydrogen peroxide solution $(30 \%)$, ethanol $(99.5 \%)$, acetone $(99.9 \%)$, and potassium hexacyanoferrate (III) were purchased from Sigma-Aldrich and used as received. MCE filter papers of varying pore sizes of 25-, 50- and 100-nm (47-mm diameter and 105- $\mu \mathrm{m}$ thickness) were purchased from Millipore. Bare gold slides (50-nm thickness), and fastdrying silver paint were purchased from Ted Pella. 99\% metallic single-walled carbon nanotubes (M-SWCNT) and PureTube Carbon Nanotubes (PSWCNTs) solutions were purchased from Nanointegris. All solutions were prepared with distilled water (18.2 M $\Omega$ $\mathrm{cm}^{-1}$ ) from a Milli-Q Direct-8 water system.

3.2.2 Instrumentation: All electrochemical measurements were performed on a $\mathrm{CHI}$ 700D Series electrochemical system with a three-electrode setup in a $1 \mathrm{~mL}$ voltammetric cell at room temperature. Our porous gold film was used as working electrode, with $\mathrm{Ag} / \mathrm{AgCl}$ as a reference electrode and platinum wire as counter electrode. Cyclic voltammetry experiments were carried out at a scan rate of $10 \mathrm{mV} / \mathrm{s}$ over the relevant potential range between $-0.1 \mathrm{~V}$ and $0.75 \mathrm{~V}$ in $0.1 \mathrm{M}$ PBS ( $\mathrm{pH} 7.4)$. Sheet resistance was measured using a Keithly source meter 4200 with four-probe setup. Electron transfer rate measurements of the porous gold film and the bare gold slide were collected using a $\mathrm{K}_{3} \mathrm{Fe}(\mathrm{CN})_{6}{ }^{3-} / \mathrm{K}_{3} \mathrm{Fe}(\mathrm{CN})_{6}{ }^{4-}$ redox probe in aqueous solution. All measurements and 
experiments were carried out at room temperature. Energy dispersive spectroscopy (EDS) was coupled to scanning electron microscopy (SEM) with a JEOL-JSM 5900 LV/EDAX to determine the composition of various films. Scanning electron microscopes (MERLIN, Carl Zeiss, Oberkochen, Germany) were used for the SEM analysis. SEM images of the film surface were obtained at the accelerating voltage of $3 \mathrm{kV}$, and JEOL JSM-6330F Field Emission Scanning Electron Microscope (FE-SEM) at an accelerating voltage of $200 \mathrm{kV}$ was used for comprehensive cross-section imaging. Contact angle measurements were performed by measuring the apparent contact angle of water $(\theta \mathrm{w}, \mathrm{a})$ using the KYOWA interface Measurement and Analysis System FAMAS - DropMaster (DM)CE1 instrument. The drop volume of $4.0 \pm 1 \mu \mathrm{L}$ (Millipore18.2 $\mathrm{M} \Omega \mathrm{cm}^{-1}$ DI water) was used in the measurement of contact angle using sessile drop method in time function for a period of 10 seconds with measurements performed at one-second intervals using FAMAS software utilizing both circular and tangent method drop fitting. Atomic force microscopy (AFM) topography images of various films were acquired in the tapping mode using a Multimode AFM IIID (Veeco Instruments) using Bruker AFM probes (TESP-V2; 0.01-0.025 Ohm/cm Antimony (n) doped Si). AFM was also used to measure the thickness of the AuNP/M-SWCNT hybrid film. During the measurement, the AFM tip scans across the lateral edge between the hybrid film and the bare glass substrate and the vertical distance (height) between the step of hybrid film and the bare glass substrate was determined. The scan range is more than $50 \mu \mathrm{m}$ to avoid the inaccuracy originating from the difference between the hybrid film's edge and its inner part. Several points have been measured to get an average height of the hybrid film with a scan rate of $0.5 \mathrm{~Hz}$. 
3.2.3 Preparation of colloidal gold nanoparticle solution: Prior to the synthesis of the gold nanoparticle solution, all glassware was incubated overnight at room temperature in $\mathrm{HNO}_{3}-\mathrm{HCl}(3: 1 \mathrm{v} / \mathrm{v})$, and washed with distilled, deionized (DI) water. Fren's citrate reduction of $\mathrm{HAuCl}_{4}$ was used to prepare the AuNPs ${ }^{166}$ with sodium citrate acting as both a stabilizing and reducing agent. Boiling DI water $(96 \mathrm{~mL})$ was mixed with freshly prepared and filtered $0.1 \mathrm{M} \mathrm{HAuCl}_{4} 3 \mathrm{H}_{2} \mathrm{O}(2.5 \mathrm{~mL}$ in DI water $)$ in a round bottom flask under vigorous stirring. Then, $34 \mathrm{mM}$ aqueous sodium citrate (2 $\mathrm{mL}$ in DI water) solution was rapidly added into the mixture at once with continuous stirring to reduce the oxidized $\mathrm{Au}^{3+}$ species to form gold nanoparticles with diameter $\sim 12 \pm 1 \mathrm{~nm}$. Once the solution changed color to red, it was kept boiling for another $30 \mathrm{~min}$ and then cooled down to room temperature and filtered using a $0.22 \mu \mathrm{m}$ syringe filter for immediate use. The solution was analyzed with a Cary 100 Bio UV-Vis spectrophotometer to establish the characteristic absorbance peak at $520 \mathrm{~nm}$.

3.2.4 Production of VF-fabricated gold films: $8 \mu \mathrm{g}$ of $99 \% \mathrm{M}-\mathrm{SWCNT}$ solution (1 $\mathrm{mg} / 100 \mathrm{~mL}$ as stock concentration) was dispersed in $1 \%$ Triton X-100 with a Fisher Scientific FS-60D sonication system for 15 min prior to use. A Kontes Ultra-Ware vacuum filtration setup was used for the film preparation. MCE filter paper was moistened and washed with deionized (DI) water. $8 \mu \mathrm{g} / 10 \mathrm{~mL}$ of the dispersed MSWCNT solution was taken as the initial feed and added to the moistened filter paper under vacuum conditions to form a thin, uniform layer on the surface at a flow-rate of 0.6 $\mathrm{mL} /$ minute. This film was washed with DI water to remove the Triton X-100 surfactant. The film was then left to vacuum dry for about $5 \mathrm{~min} .35$ picomoles $/ 10 \mathrm{~mL}$ of citratecapped AuNP solution (3.5 $\mathrm{nM}$ as stock concentration) was added on top of the M- 
SWCNT underlayer to form a gold film at a flow-rate of $6 \mathrm{~mL} /$ minute. This VFfabricated gold film was washed with DI water and vacuum dried overnight. After complete drying, the hybrid gold film was cut into 14 strips $(5 \mathrm{~mm} \mathrm{~W} \times 8 \mathrm{~mm} \mathrm{~L})$, using a cutting template to ensure reproducible results. These strips were connected to copper wires with fast-drying silver paste (Ted Pella Inc. USA) and wrapped around with Parafilm to form the working electrodes (Figure 2). Purchased gold slides were cut to have the same geometric surface area as the hybrid gold film electrode and cleaned with piranha solution before use.

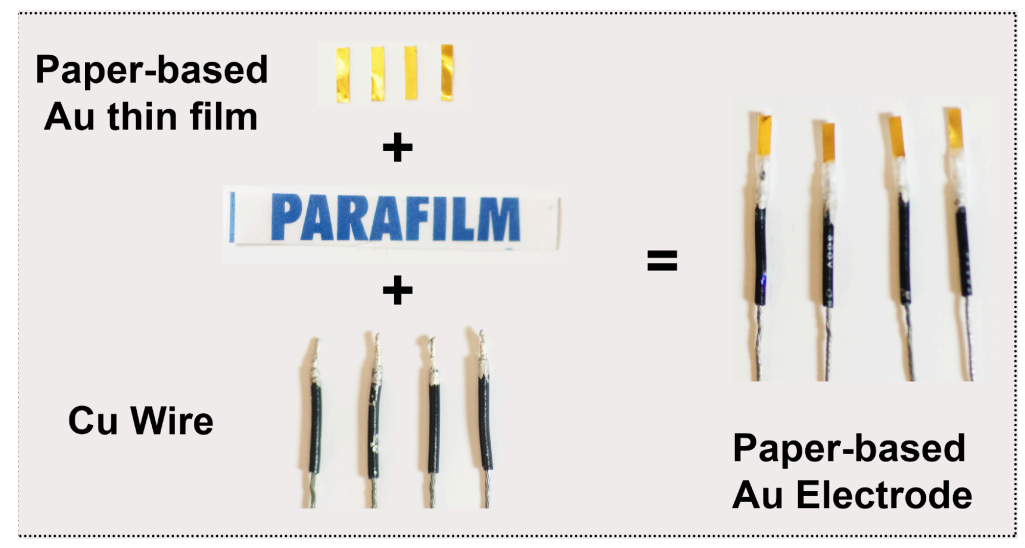

Figure 2: Schematic illustration of the preparation of working VF-fabricated gold film electrodes. The strip electrodes were attached to copper wires using fast drying silver paint for electrical contact and wrapped around with Parafilm.

3.2.5 Alternative VF-fabricated gold film compositions: We also fabricated hybrid gold films with MCE filter papers of varying pore sizes of 25-, 50- and 100-nm, with AuNPs of varying diameters, and with different amounts of PureTube Carbon Nanotubes (P-SWCNTs). 
First, we produced VF-fabricated gold films on MCE filter papers with different pore sizes $(25,50$, and $100 \mathrm{~nm})$. Eight $\mu \mathrm{g}$ of M-SWCNT and 35 picomoles of AuNPs were sequentially added as described above. Dense and uniform films with improved conductivity were formed with 25 - and 50-nm filter papers in comparison with the 100$\mathrm{nm}$ pore MCE, with a decrease in sheet resistance from $113 \Omega / \mathrm{sq}$ with the $100-\mathrm{nm}$ membrane to $37 \Omega / \mathrm{sq}$ with the $25-\mathrm{nm}$ membrane (Figure $3 \mathrm{~A}$ ). This decrease in sheet resistance was correlated with a $52 \%$ increase in electron transfer rate from $2.76 \times 10^{-3}$ $\mathrm{cm} / \mathrm{s}$ to $5.74 \times 10^{-3} \mathrm{~cm} / \mathrm{s}$ for the 100 - and $25-\mathrm{nm}$ films (Figure $3 \mathrm{~B}$ ), respectively. Dense packing of M-SWCNTs on the smaller paper pore sizes (25- and 50-nm) may be responsible for the improved conductivity of these films in comparison with the 100-nm film.

A

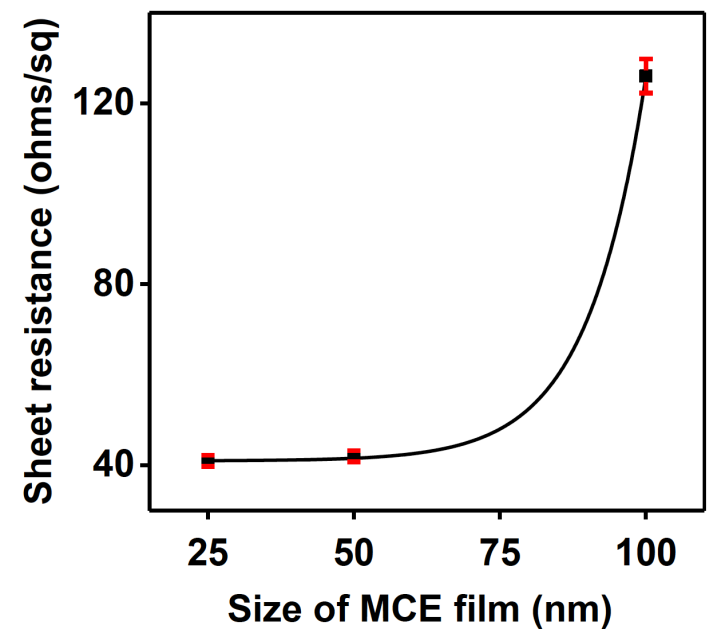

B

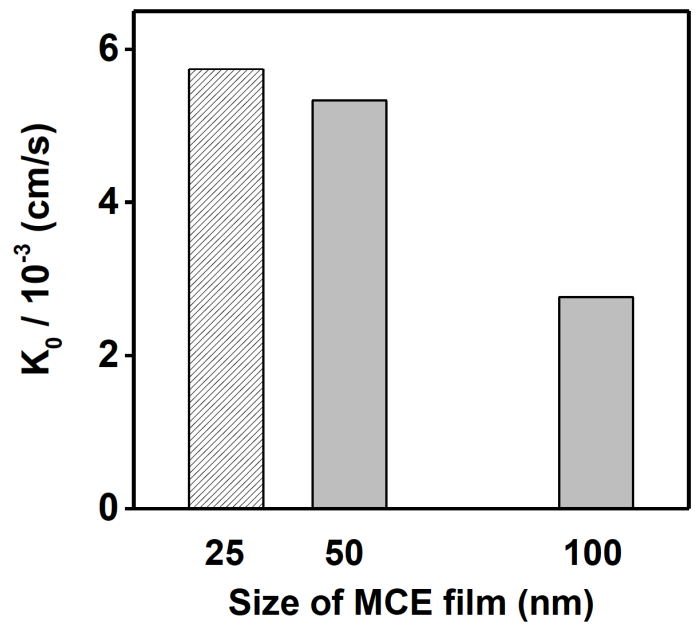

Figure 3: Effect of pore-size of MCE filter papers on sheet resistance and electron transfer rate. (A) VF-fabricated gold films with smaller pores $(25$ and $50 \mathrm{~nm})$ achieved dense packing of MSWCNTs on the MCE filter paper, which enabled formation of a dense and smooth gold film with a sheet resistance of $35 \mathrm{ohms} / \mathrm{sq}$, relative to 126 $\mathrm{ohms} / \mathrm{sq}$ sheet resistance obtained with 100-nm MCE film. (B) An increase of electron transfer rates was also observed with decreasing pore size, which enabled denser packing of M-SWCNTs. 
Next, we synthesized AuNPs with diameters of 30, 54, and $75 \mathrm{~nm}$ using this kineticallycontrolled seed-mediated growth process, with sequential addition of gold salt solution and sodium citrate. ${ }^{167}$ The molar extinction coefficients, concentrations and sizes of the synthesized AuNPs were roughly estimated based on previously published work ${ }^{168}$ The concentration of these AuNPs were normalized relative to the concentration of our 13-nm AuNPs $(3.5 \mathrm{nM})$ to adjust the volumes appropriately to form a thin layer of AuNPs on top of the M-SWCNT underlay. Finally, we prepared hybrid gold films with varying amounts of P-SWCNTs. We prepared $10 \mathrm{~mL}$ solutions containing 12.5, 25, 50, and 100 $\mu \mathrm{g}$ of P-SWCNTs solution ( $25 \mathrm{mg} / 100 \mathrm{~mL}$ as stock concentration), which were dispersed in $1 \%$ Triton X-100 with a Fisher Scientific FS-60D sonication system for 15 min prior to use. MCE filter paper of $100 \mathrm{~nm}$ pore size was moistened and washed with DI water. The dispersed P-SWCNT solution was added to the moistened filter paper under vacuum conditions to form a thin, uniform layer on the surface at a flow-rate of $0.6 \mathrm{~mL} / \mathrm{minute}$. This film was washed with DI water to remove the residual Triton X-100 surfactant. The film was then left to vacuum dry for about $5 \mathrm{~min} .35$ picomoles $/ 10 \mathrm{~mL}$ of citrate-capped AuNP solution was then added to form a smooth gold film at a flow-rate of $6 \mathrm{~mL} / \mathrm{minute}$. This VF-fabricated gold film was further washed with DI water and vacuum dried overnight. An estimated cost for the current system is $\sim \$ 0.79 / \mathrm{cm}^{2}$ when M-SWCNTs are used as the underlayer. However, we have also demonstrated the feasibility of replacing this relatively expensive M-SWCNT underlayer with much cheaper P-SWCNTs without sacrificing conductivity or electrocatalytic activity, and this effectively brings down the cost to $\sim \$ 0.03 / \mathrm{cm}^{2}$. 


\subsubsection{Template-free (TF) electrodeposition of AuNPs on paper-based M-SWCNT}

film: Electrodeposition was performed using a three-electrode setup in a $1 \mathrm{~mL}$ cell using a CHI760 electrochemistry station. Paper-based M-SWCNT films (8 or $40 \mu \mathrm{g}$ ) prepared as described above served as the working electrode, whereas $\mathrm{Ag} / \mathrm{AgCl}$ and $\mathrm{Pt}$ wires were used as the reference and counter electrodes, respectively. Gold nanoparticles were electrochemically deposited on the paper-based M-SWCNT film layer by cyclic voltammetry, ${ }^{169}$ scanning from $1.25 \mathrm{~V}$ to $-1.0 \mathrm{~V}$ in $0.01 \mathrm{M} \mathrm{NaNO}_{3}$ containing $1 \mathrm{mM}$ $\mathrm{HAuCl}_{4}$ at a scan rate of $50 \mathrm{mV} / \mathrm{s}$ for 120 cycles.

\subsubsection{Hydrogen bubble (HB) - directed electrodeposition of AuNPs on paper-based} M-SWCNT film: Hydrogen bubbles were used as a template to perform electrodeposition of $\mathrm{AuNPs}^{46}$ in a three-electrode setup in a $1 \mathrm{~mL}$ cell using a CHI760 electrochemistry station. Hydrogen bubble-directed electrodeposition of AuNPs was performed by chronopotentiometry, with paper-based M-SWCNT film layer (8 or $40 \mu \mathrm{g})$ prepared as described above as a working electrode and with $\mathrm{Ag} / \mathrm{AgCl}$ and $\mathrm{Pt}$ wires used as the reference and counter electrodes, respectively. An aqueous solution consisting of $0.1 \mathrm{M} \mathrm{HAuCl}_{4}$ and $3 \mathrm{M} \mathrm{NH}_{4} \mathrm{Cl}$ was used to deposit AuNPs in the potential range of 1 to $-8 \mathrm{~V}$, with a fixed cathodic current of 0.007 A over $120 \mathrm{~s}$.

3.2.8 Contact angle measurements on various surface: Contact angle measurements were performed by measuring the apparent contact angle of water $\left(\theta_{\mathrm{w}, \mathrm{a}}\right)$ using the KYOWA interface Measurement and Analysis System FAMAS - DropMaster (DM)CE1 instrument on four different substrates. Commercially purchased gold slide and MCE filter paper (100-nm pore size) were used as controls in the measurement of contact angles of our M-SWCNT and M-SWCNT/AuNPs hybrid films. The drop volume of $4.0 \pm$ 
$1 \mu \mathrm{L}$ (Millipore18.2 $\mathrm{M} \Omega \mathrm{cm}^{-1}$ DI water) was used in the measurement of contact angle using sessile drop method in time function for a period of 10 seconds with measurements performed at one-second intervals using FAMAS software utilizing both circular and tangent method drop fitting. MCE filter paper, M-SWCNT, and M-SWCNT/AuNPs hybrid films were stored in the lab atmosphere prior to the contact angle measurements whereas commercial gold slides were thoroughly cleaned with acetone, ethanol, DI water, and piranha solution and dried in vacuum.

\subsubsection{Determination of surface roughness of fabricated gold films using cyclic}

voltammetry: A three-electrode setup was used to cycle the potential between -0.5 to 1.5 $\mathrm{V}$ in $0.5 \mathrm{M} \mathrm{H}_{2} \mathrm{SO}_{4}$ at a scan rate of $50 \mathrm{mV} / \mathrm{s}$ using the as-prepared gold film working electrode, $\mathrm{Ag} / \mathrm{AgCl}$ reference electrode, and Pt wire counter electrode. Reduction of the gold oxide peak at $\sim 0.8 \mathrm{~V}$ was integrated to obtain the charge. We used the integrated charge to calculate the electroactive area and roughness factor of the film, assuming that $386 \mu \mathrm{C} / \mathrm{cm}^{2}$ was required to reduce a single monolayer of gold oxide. ${ }^{170}$

\subsection{Results and Discussions}

Our porous gold films prepared in layer-by-layer fashion using a simple vacuum filtration apparatus offers excellent stability, good homogeneity, and reproducible with strong adhesive strength between the layers. The choice of the substrate such as mixed cellulose ester (MCE) filter membrane possesses many useful characteristics being biodegradable, combustible, eco-friendly, cheap, and portable, with the flexibility and strength to allow multiple sample loadings and washing steps without sacrificing surface integrity. Citrate-

capped AuNPs $(3.5 \mathrm{nM}, 12 \pm 1-\mathrm{nm}$ diameter $)$ were synthesized as reported previously ${ }^{166}$ ahead of our composite film fabrication. Transmission electron microscopy (TEM) and 
image analysis confirmed that the AuNPs were uniform in size, with an average diameter of $\sim 12 \pm 1$-nm with good homogeneity (Figure 4).

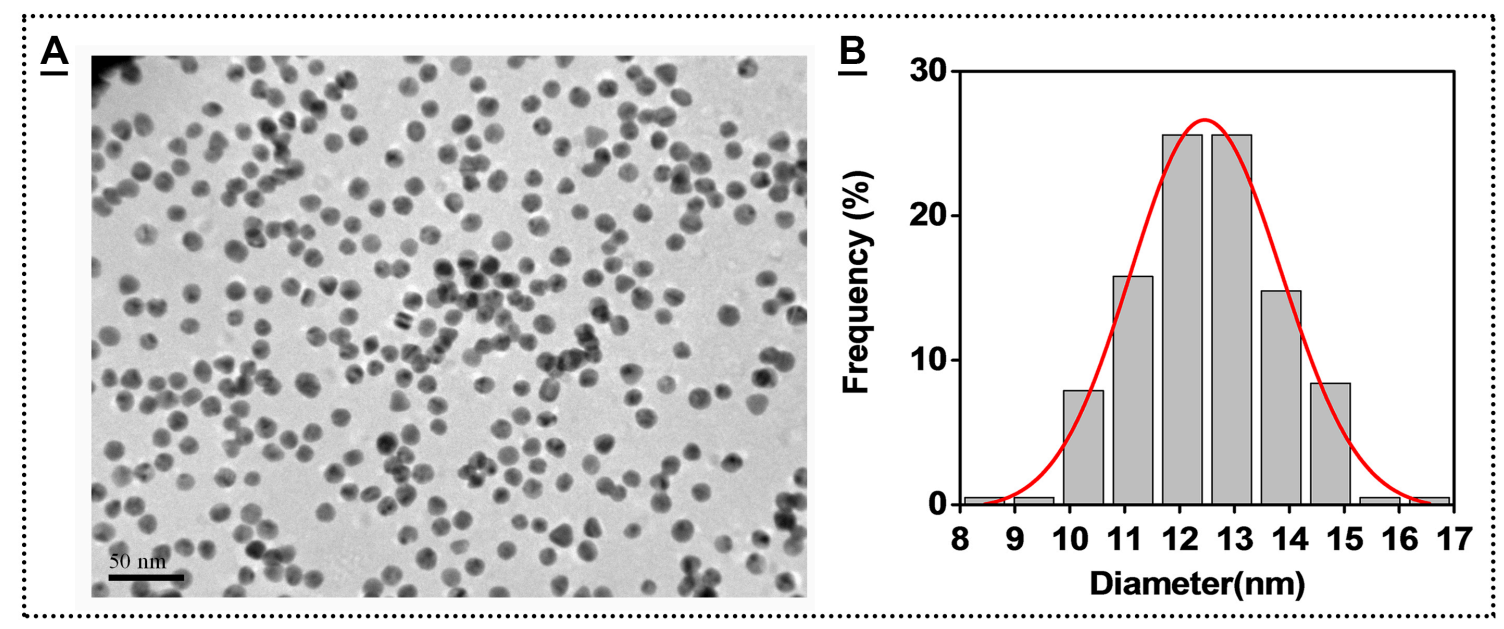

Figure 4: Transmission electron microscopy (TEM) image of (A) citrate-capped AuNPs using a Phillips CM-200 at $200 \mathrm{kV}$ reveals roughly spherical, homogenous particles. (B) Frequency distribution histogram shows an average particle diameter of $12 \pm 1 \mathrm{~nm}$, as calculated using Image J software.

8 ug of $99 \%$ M-SWCNT stock solution was dispersed in $10 \mathrm{~mL}$ of $1 \%$ Triton X-100 surfactant via sonication for $10 \mathrm{~min}$. The film was prepared in three steps (Figure 5). First, we performed vacuum filtration of the M-SWCNT suspension through a Buchner funnel onto MCE paper to form a uniform, compact, randomly-aligned M-SWCNT layer (Figure 5A). A low vacuum pressure was maintained at a flow-rate of $0.6 \mathrm{~mL} / \mathrm{min}$ to trap most of the M-SWCNTs on the filter paper. After washing away the Triton X-100 surfactant from the M-SWCNT layer (Figure 5B), we performed a second vacuum filtration step with $10 \mathrm{~mL}$ of citrate-capped AuNP solution (35 picomoles). During this step, the AuNP solution was quickly drawn through the M-SWCNT-layered MCE paper at a fast flow-rate $(6 \mathrm{~mL} / \mathrm{min})$ to avoid aggregation of AuNPs on the film surface. Once the second filtration step was complete, a gold film had formed atop the M-SWCNT underlay (Figure 5C). 


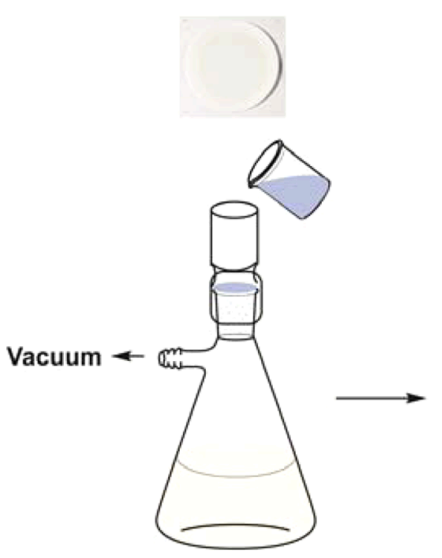

A: M-SWCNT loaded
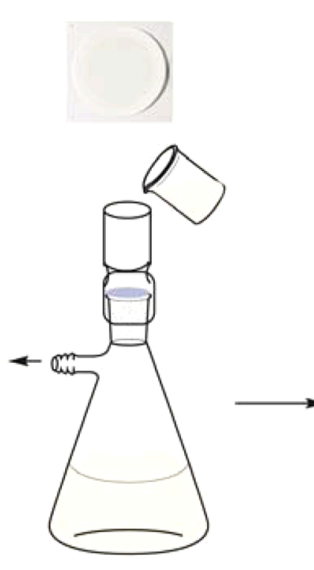

B: DI washing

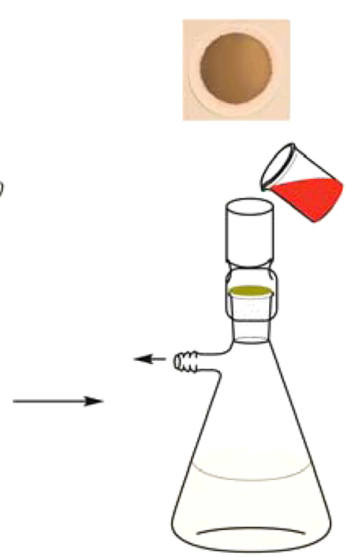

C: AuNP loaded

Figure 5: Porous thin gold film preparation via vacuum filtration. (A) Vacuum filtration of a solution of $1 \%$ Triton X-100-dispersed M-SWCNTs results in a compact and uniform M-SWCNT underlay on the paper surface. (B) Triton X-100 surfactant is washed away with copious amounts of deionized (DI) water to provide a clean hydrophobic surface for the deposition of AuNPs on top of the M-SWCNT layer. (C) Subsequent vacuum filtration of a citrate-capped AuNP solution yields a highly conductive, AuNP/M-SWCNT hybrid thin film on the MCE paper.

In light of the strategy for fabricating the hybrid gold film, we believe that the vacuum force is an essential source of binding between the citrate-capped AuNPs and the MSWCNTs deposited on the filter paper. Since the Triton X-100 surfactant is washed away from the M-SWCNT layer with copious amounts of DI water after depositing the MSWCNTs on the paper, we trust that the suction produced by the vacuum force is instrumental in forming hydrophobic-hydrophobic interactions between the M-SWCNTs and citrate-capped AuNPs. Vacuum filtration drives the all-around scattered M-SWCNTs to form a compact, arbitrarily displayed layer on the paper surface with greatest cover and interpenetration, yielding moderate conductivity and great mechanical strength all through the rigid film. Rapid filtration of the AuNP solution, in turn, forces the deposition of the particles onto the surface of the M-SWCNT layer, forming a highly conductive hybrid thin gold film. The subsequent thickness and physical properties of the 
film can be controlled at the nanoscale by essentially changing either the concentration or the feed volume of the nanomaterials. This procedure brings about a conductive AuNP/M-SWCNT onto the filter paper.
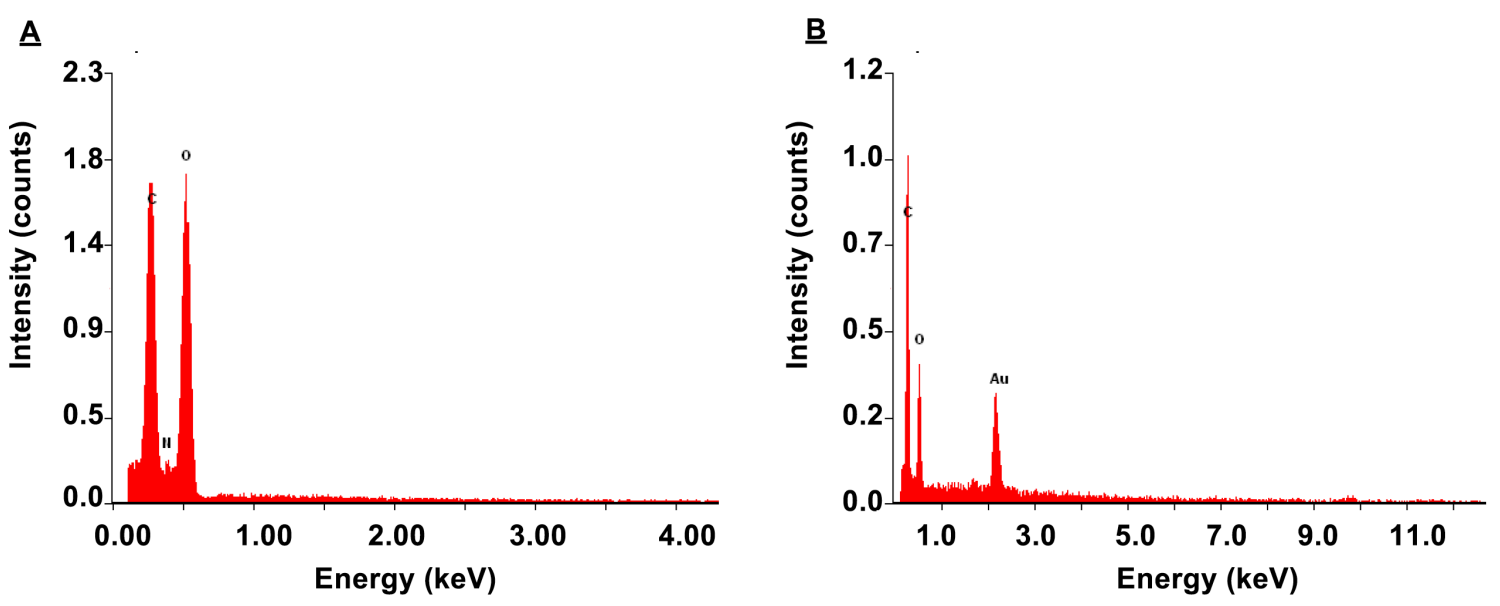

Figure 6: X-ray energy dispersive spectroscopy (EDS) of (A) a single layer of MSWCNTs on MCE or (B) an AuNP/M-SWCNT hybrid film on MCE. Besides carbon, nitrogen, and oxygen no other element peaks were seen for the M-SWCNT film, confirming the absence of any metal catalyst impurity. These results confirmed a high $\mathrm{Au}$ content (the mass ratio of $\mathrm{Au} / \mathrm{C}$ is about $25 \%$ ) for the hybrid film, with no other detectable elements except for carbon and oxygen, demonstrating its purity.

To assess the presence of impurities on the VF-fabricated films, we performed an elemental analysis using X-ray energy dispersive spectroscopy (EDS) (Figure 6). Experimental results demonstrated an absence of superfluous element peaks for the MSWCNT film, except for carbon, nitrogen, and oxygen, affirming the nonattendance of any metal catalyst impurity (Figure 6A). It is additionally imperative that a high $\mathrm{Au}$ content was acquired with no perceivable impurities on the VF-fabricated gold film, affirming that our procedure yields a pure gold layer on the M-SWCNT film (Figure 6B). Contact angle measurements were performed on various films to assess their wettabilities (Figure 7). Clearly, the apparent contact angle of water $(\theta \mathrm{w}, \mathrm{a})$ on an M-SWCNT-only film was $81^{\circ}$, showing a hydrophilic surface. The hybrid film showed a marginally 
diminished $\theta \mathrm{w}$, a value $\left(76^{\circ}\right)$, which is somewhat higher than the $\theta \mathrm{w}$, a value $\left(71^{\circ}\right)$ of the purchased flat gold slide. In all cases, the $\theta \mathrm{w}$,qualities were higher contrasted with the MCE paper substrate $\left(56^{\circ}\right)$.

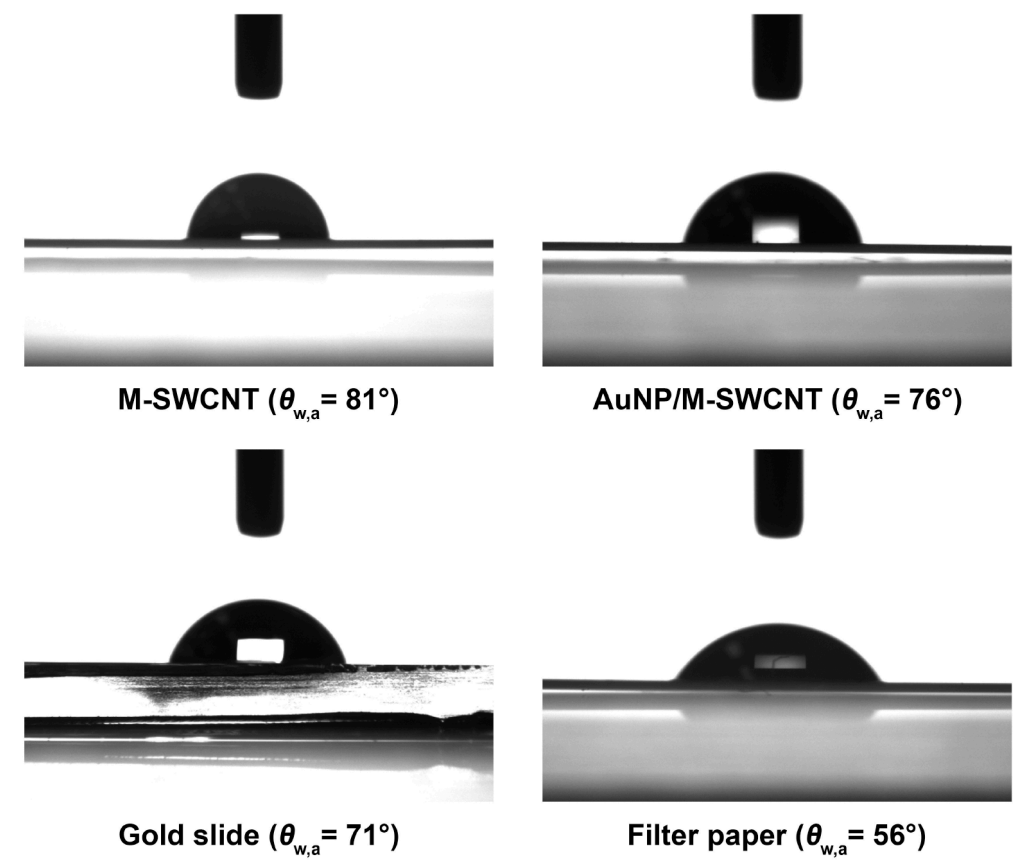

Figure 7: Apparent contact angle of water $(\theta \mathrm{w}, \mathrm{a})$ for the surface of various films.

To better understand the synergistic effect of the nanostructure of both layers on our film's conductivity, we performed the measurements of sheet resistance $(\Omega / \mathrm{sq})$ of various films (Table 1) with a Keithly 4200 Semiconductor Characterization System with a fourpoint probe setup. As expected, the MCE filter paper was not conductive, and we observed infinite resistance during measurement. When 35 picomoles of citrate-coated AuNPs were directly applied onto the filter paper using filtration with no M-SWCNT underlayer, we observed spillage of AuNPs through the membrane pores, producing a pink coating on the paper that demonstrated no conductivity. When we expanded the thickness of the gold coating by adding 5 layers of 35 picomoles of citrate-capped AuNPs, the color changed from pink to wine red yet at the same time displayed no 
measurable conductivity, potentially because of the vast interstitial spaces among the AuNPs.

Table 1: Sheet resistance $(\mathrm{Ohm} / \mathrm{sq})$ and calculated electron transfer rate $\left(\mathrm{K}_{\mathrm{o}}\right)$ for different films.

\begin{tabular}{|c|c|c|}
\hline Type of film & $\begin{array}{l}\text { Sheet Resistance } \\
\qquad(\Omega / \mathrm{sq})\end{array}$ & $\mathrm{K}_{\mathrm{o}}(\mathrm{cm} / \mathrm{s})$ \\
\hline A: MCE filter paper & $\mathrm{N} / \mathrm{A}$ & $\mathrm{N} / \mathrm{A}$ \\
\hline B: Single layer of AuNPs on MCE & $N / A$ & $N / A$ \\
\hline C: Multiple layers of AuNPs on MCE & $\mathrm{N} / \mathrm{A}$ & $\mathrm{N} / \mathrm{A}$ \\
\hline D: Single layer of M-SWCNTs on MCE & 75,000 & $\mathrm{~N} / \mathrm{A}$ \\
\hline $\begin{array}{l}\text { E: M-SWCNT/AuNP hybrid film on MCE } \\
\text { (M-SWCNTs as underlay) }\end{array}$ & 126 & $2.73 \times 10^{-3}$ \\
\hline F: Commercially available bare gold slide & 20 & $4.92 \times 10^{-3}$ \\
\hline
\end{tabular}

Conversely, $8 \mu \mathrm{g}$ of M-SWCNTs formed an overlapping network on the paper due to their micro-scale length, giving cross-connected conductive channels to electron exchange and in this manner making the film tolerably conductive $(\sim 75,000 \mathrm{Ohm} / \mathrm{sq})$. Such a thin underlay of M-SWCNTs offered a rigid mesh infrastructure that kept individual AuNPs from evading amid a consequent round of AuNP filtration. This was clearly evidenced by the immense conductivity observed for films comprising 35 picomoles of AuNPs deposited on an underlay of $8 \mu \mathrm{g}$ M-SWCNTs. The sheet resistance of this AuNP/M-SWCNT hybrid thin film was $126 \mathrm{ohm} / \mathrm{sq}, \sim 600$-fold lower than that of the M-SWCNT underlay alone. The significant increment in conductivity is conceivable because of the way that the AuNPs were either in contact with the M-SWCNTs or filling the holes in the interconnecting M-SWCNT network to shape an all-around interconnected network. Because of the porosity of our VF-fabricated hybrid film, the conductivity is six-fold lower on the purchased gold slides (20 ohms/sq) (Table 1). We 
believe that the M-SWCNT layer acts as both a conducting bed and a support matrix. To show the commitment of M-SWCNTs as a conductive layer, we fabricated films composed only of various measures of M-SWCNTs. These films were moderately conductive, and increasing amounts of M-SWCNTs resulted in a decline in sheet resistance; a sheet resistance of $13,049 \Omega / \mathrm{sq}$ was acquired from the film fabricated with $20 \mu \mathrm{g}$ M-SWCNT (Figure 8).

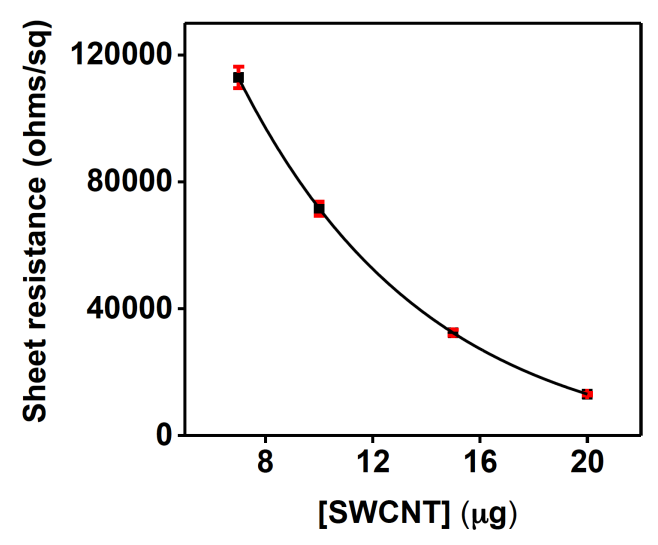

Figure 8: Effect of variable amounts of M-SWCNTs on sheet resistance in the absence of AuNPs. A gradual decline in sheet resistance can be observed with increasing amounts of M-SWCNT from 7-20 $\mu \mathrm{g}$.

The M-SWCNTs also play a critical role as a rigid support and barrier layer for the AuNPs in the fabrication of the composite hybrid film, keeping them from going through the filter paper substrate. In parallel, the AuNPs decrease the contact resistance between the M-SWCNTs by acting as nanofillers that are physically attached at the inter-tube junctions, in this manner forming a conductive three-dimensional electron exchange pathway that incredibly increases the conductivity of the hybrid film. To demonstrate this, we measured the effect of variable amounts of M-SWCNTs with an upper layer of 35 picomoles of AuNPs on the sheet resistance of hybrid film. We found that the film's sheet resistance decreased from $571 \Omega /$ sq to $342 \Omega /$ sq (Figure 9) as we increased the M- 
SWCNT underlayer from $4.4 \mu \mathrm{g}$ to $5.3 \mu \mathrm{g}$, and further increments in the amount of MSWCNTs brought about a relentless diminishing in sheet resistance until bottoming out at $17.8 \mu \mathrm{g}$ of M-SWCNTs with a sheet resistance of $21 \Omega / \mathrm{sq}$.

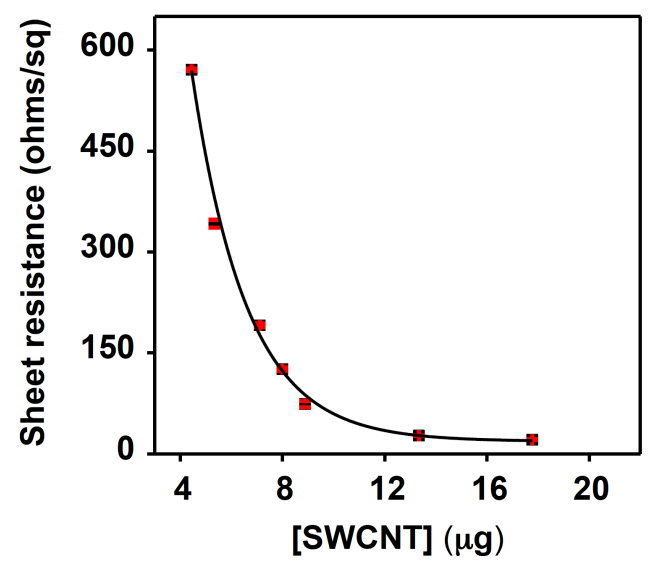

Figure 9: Effect of variable amounts of M-SWCNTs on sheet resistance in the presence of 35 picomoles AuNPs. Increasing amounts of M-SWCNTs (4.4-17.8 $\mu \mathrm{g}$ ) with an upper layer of 35 picomoles AuNPs result in a steady decline in sheet resistance, with values starting to bottom out at around $8 \mu \mathrm{g}$.

We then demonstrated the contribution of the AuNPs to the hybrid film conductivity by fabricating films with $8 \mu \mathrm{g}$ M-SWCNTs and distinctive amounts of AuNPs (Figure 10). Compared with the sheet resistance of 75,000 $\Omega$ /sq acquired from a M-SWCNT-only film, we observed that the sheet resistance of the AuNP/M-SWCNT hybrid film decreased 51-fold upon loading only 14 picomoles of AuNPs $(1,469 \Omega / \mathrm{sq})$ and then additionally decreased to $230 \Omega /$ sq with 28 picomoles AuNPs. Resistance bottomed out at $67 \Omega / s q$ with 53 picomoles AuNPs. The relatively high resistance of films formed from 14 picomoles or less of AuNPs is apparently because the amount of AuNPs was just adequate to yield discrete patches of particles anchored to the sidewalls of the MSWCNTs, whereas increasing the amount of AuNPs to 28 picomoles or higher allows the particles to accumulate and form a continuous AuNP film with enhanced conductivity. 


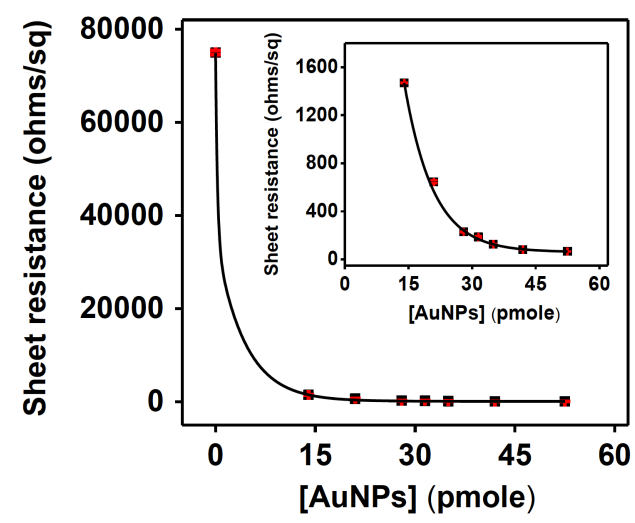

Figure 10: Effects of variable amounts of AuNPs on sheet resistance with fixed amount of M-SWCNTs. Increasing amounts of AuNPs with an underlayer of $8 \mu \mathrm{g}$ M-SWCNT resulted in a steady decline in sheet resistance, with values starting to bottom out at around 28 pmole. The inset shows the declining trend more clearly, without the $8 \mu \mathrm{g} M-$ SWCNT-only control.

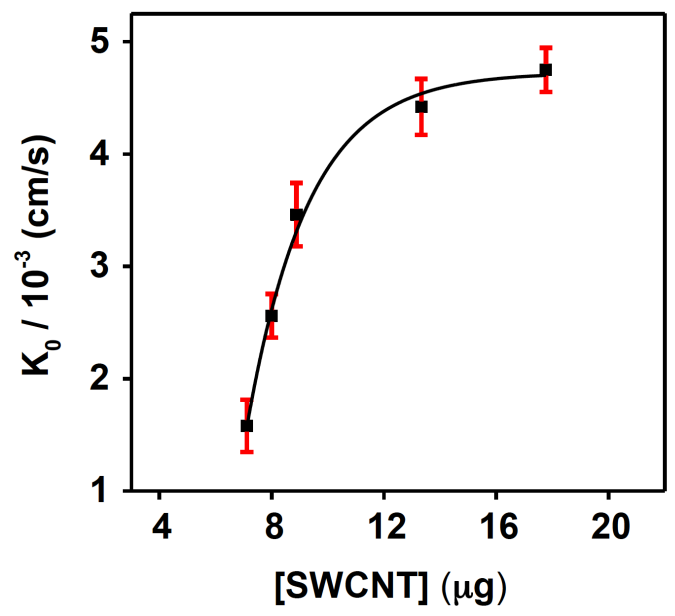

Figure 11: Effect of variable amounts of M-SWCNTs on electron transfer rate with fixed amount of AuNPs (35 pmoles). Increasing amounts of M-SWCNTs as an underlayer for 35 pmoles of AuNPs were associated with a steady increase in electron transfer rate $\left(\mathrm{K}_{0}\right)$, with values starting to reach a plateau around $13.3 \mu \mathrm{g}$.

We further characterized the electron transfer rate constants of these various films. The electron transfer rate is depicted as the procedure of exchange of electrons between the surface of the working electrode and its interfacial solution. It can be calculated by examining the peak-to-peak separation $(\Delta \mathrm{Ep})$ of $\mathrm{Fe}(\mathrm{CN})_{6}{ }^{3-} / \mathrm{Fe}(\mathrm{CN})_{6}{ }^{4-}$ in cyclic voltammograms using the Nicholson method, ${ }^{171}$ where a small $\Delta \mathrm{Ep}$ indicates faster 
electron transfer. As expected, both MCE filter paper and AuNP-loaded paper were nonconductive to electron transfer. The M-SWCNT film yielded a $\Delta \mathrm{Ep}$ of $393 \mathrm{mV}$, indicating a very low electron transfer rate constant, validating the sheet resistance presented in Table 1. However, the $\Delta \mathrm{Ep}$ of our AuNP/M-SWCNT hybrid thin film was reduced to $85 \mathrm{mV}$, with a calculated electron transfer rate of $2.73 \times 10^{-3} \mathrm{~cm} / \mathrm{s}-$ a two-fold slower than the bare purchased gold slide $\left(4.92 \times 10^{-3} \mathrm{~cm} / \mathrm{s}\right)$. The measurement of MSWCNTs added greatly affects the gold film's electron transfer rate. For instance, a top layer of 35 picomoles of AuNPs with an underlay made with 4.4 or $5.3 \mu \mathrm{g}$ of MSWCNTs yielded an unmeasurable electron transfer because of poorly characterized peak-to-peak separation. In any case, the hybrid films made with 7.1 and $8.9 \mu \mathrm{g}$ of MSWCNTs gave a well-defined peak-to-peak separation, with calculated electron transfer rates of $1.67 \times 10^{-3} \mathrm{~cm} / \mathrm{s}$ and $2.73 \times 10^{-3} \mathrm{~cm} / \mathrm{s}$, respectively. This rate started to level with gold films fabricated with $13.3 \mu \mathrm{g}$ of M-SWCNTs, which yielded a calculated electron transfer rate of $4.33 \times 10^{-3} \mathrm{~cm} / \mathrm{s}$ (Figure 11). Obviously, the large pores that formed in layers made from small amounts of M-SWCNTs permitted many AuNPs to escape through the film, forming a poorly-conductive pink coating on the paper (Figure 12, top B-C). As we increased the amount of M-SWCNTs above $8 \mu \mathrm{g}$, most of the AuNPs were held on the thick M-SWCNT film due to the smaller pores formed within this underlay, enabling the formation of a highly conductive gold film (Figure 12, top E-H).

The increase in electron transfer rate with increasing amounts of M-SWCNTs might be viewed as a transition from dispersed particles to an accumulated single layer of AuNPs, coming about because of a diminishment in the pore size of the M-SWCNT underlayer. The side-angle view illustrates the shiny nature of the paper-based gold films, which is 
particularly clear with 8 ug or more of M-SWCNTs (Figure 12, bottom E-H). Additionally, we also prepared hybrid gold films with $12.5,25,50$, and $100 \mu \mathrm{g}$ of PSWCNT solution which was dispersed in $1 \%$ Triton X-100. We observed that increasing amounts of P-SWCNTs $(12.5-75 \mu \mathrm{g})$ as an underlayer for 35 pmoles of AuNPs were associated with a decline in sheet resistance and an increment in electron transfer rate $\left(\mathrm{K}_{0}\right)$, with values starting to reach a plateau around $50 \mu \mathrm{g}$ (Figure13).
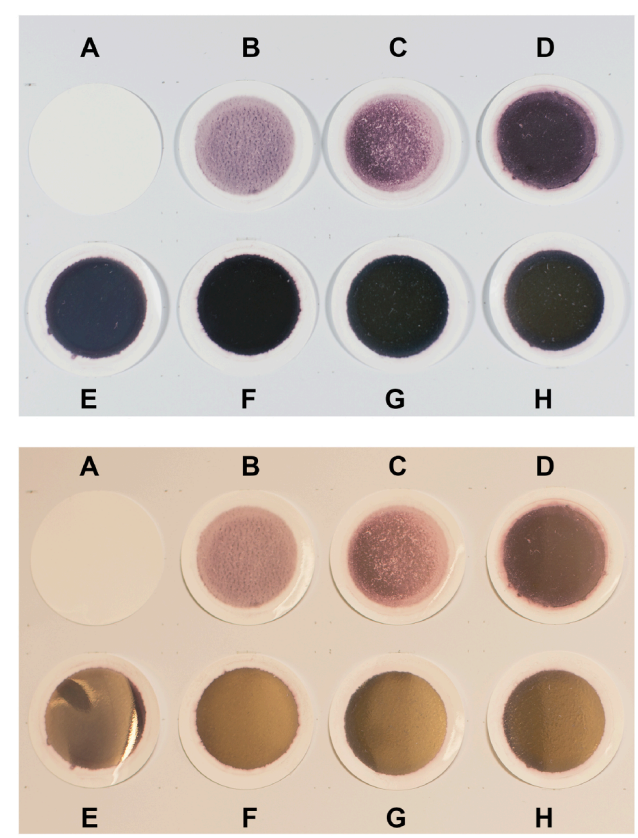

Figure 12: Front angle view (top) and side angle view (bottom) of films fabricated with 35 pmoles AuNPs and variable amounts of M-SWCNTs. (A) MCE alone, (B) $4.4 \mu \mathrm{g} \mathrm{M}-$ SWCNTs, (C) $5.3 \mu \mathrm{g}$ M-SWCNTs, (D) $7.1 \mu \mathrm{g}$ M-SWCNTs, (E) $8 \mu \mathrm{g}$ M-SWCNTs, (F) $8.9 \mu \mathrm{g}$ M-SWCNTs, (G) $13.3 \mu \mathrm{g}$ M-SWCNTs, (H) $17.8 \mu \mathrm{g}$ M-SWCNTs. The reflective nature of the film is more clearly visible from the side-angle view, especially from films made with $\geq 8 \mu \mathrm{g}$ M-SWCNTs (E-H). 

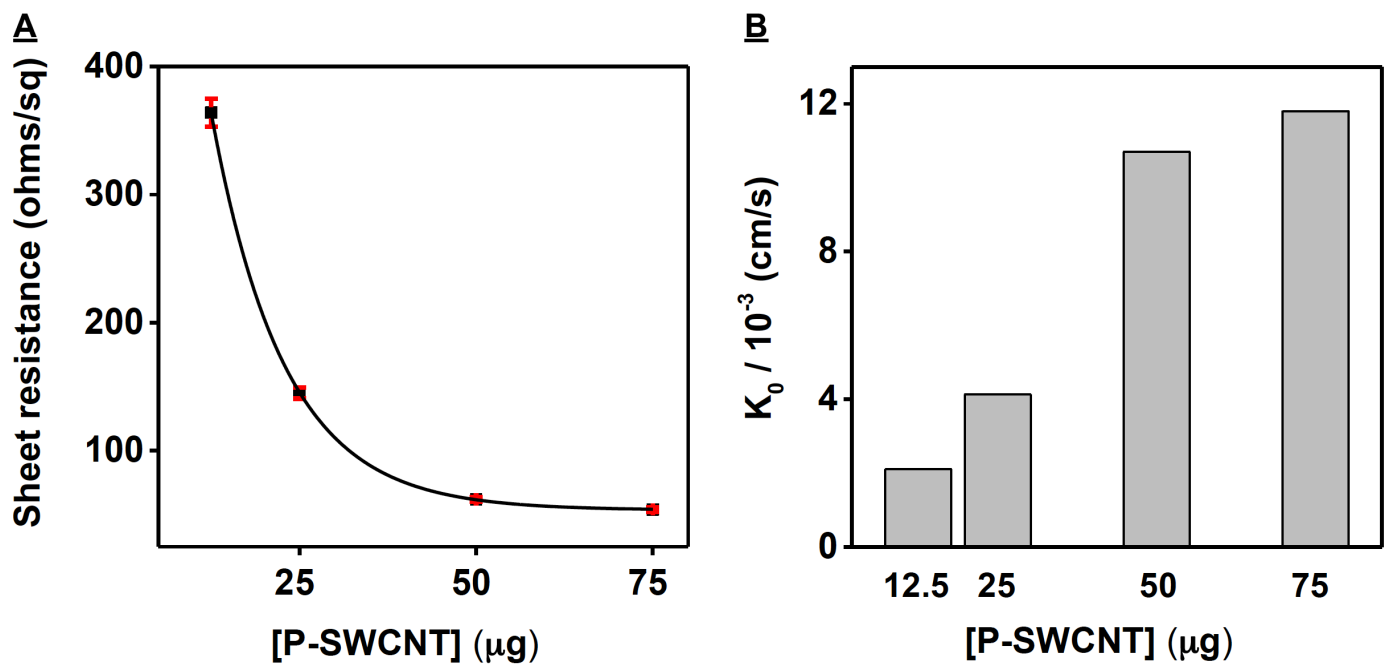

Figure 13: Effects of variable amounts of P-SWCNTs on sheet resistance and electron transfer rate. (A) Sheet resistance measurements show that increasing amounts of PSWCNTs $(12.5-75 \mu \mathrm{g})$ with an upper layer of 35 picomoles AuNPs result in a steady decline in sheet resistance, with values starting to bottom out at around $50 \mu \mathrm{g}$. (B) Electron transfer rate measurements show that increasing amounts of P-SWCNTs (12.5$75 \mu \mathrm{g})$ as an underlayer for 35 pmoles of AuNPs were associated with a steady increase in electron transfer rate $\left(\mathrm{K}_{0}\right)$, with values starting to reach a plateau around $50 \mu \mathrm{g}$.

We observed a comparable pattern about electron transfer rate as we increased the amount of AuNPs deposited on a fixed amount of M-SWCNTs. The relatively poor conductivity of the film fabricated with 14 picomoles AuNPs atop an $8 \mu \mathrm{g}$ M-SWCNT underlayer did not allow us to determine the electron transfer rate (Figure 14). However, increasing the amount of AuNPs to 28 picomoles greatly improved the film conductivity, yielding an electron transfer rate of $1.00 \times 10^{-3} \mathrm{~cm} / \mathrm{s}$. This value reached a plateau at around 35 picomoles of AuNPs (electron transfer rate of $2.73 \times 10^{-3} \mathrm{~cm} / \mathrm{s}$ ) with only small gains beyond this point (Figure 14). 


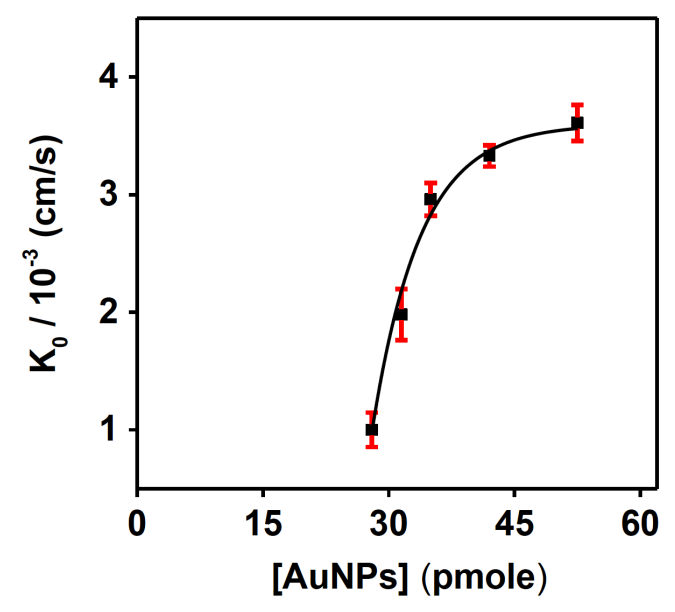

Figure 14: Effects of variable amounts of AuNPs on electron transfer rate. Starting at 28 pmole, increasing amounts of AuNPs with an underlayer of $8 \mu \mathrm{g}$ M-SWCNTs led to a steady increase in the electron transfer rate $\left(\mathrm{K}_{0}\right)$, with values starting to reach a plateau around 35 pmole.

A change in color intensity can also be clearly observed with increasing concentrations of AuNPs: pink-tinged films made with 14 or 21 picomoles of AuNPs indicate scarce dispersion of particles (Figure 15, top B-C), whereas the darker and shinier films formed with 28 picomoles or more of AuNPs (Figure 15, top D-H) suggest accumulation of AuNPs atop the M-SWCNT underlay. 

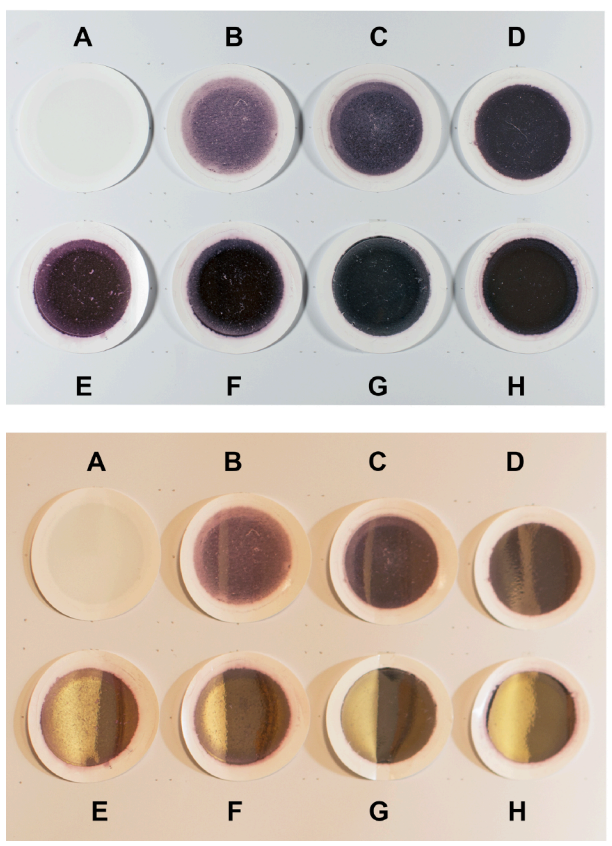

Figure 15: Front (top) and side angle view (bottom) of films fabricated with $8 \mu \mathrm{g}$ MSWCNTs and varying amounts of AuNPs. (A) $8 \mu \mathrm{g}$ M-SWCNTs alone, (B) 14 pmole AuNPs, (C) 21 pmole AuNPs, (D) 28 pmole AuNPs, (E) 32 pmole AuNPs, (F) 35 pmole AuNPs, (G) 42 pmole AuNPs, (H) 53 pmole AuNPs. The reflective nature of the film is more clearly visible from the side-angle view, especially from films made with $\geq 32$ pmole AuNPs (E-H).

Furthermore, different sizes of AuNPs $(\sim 13,30,54$, and $75 \mathrm{~nm})$ were also used to form a thin layer of AuNPs on top of the M-SWCNT underlay. We observed a gradual decrease in the electron transfer rate of films prepared from larger AuNPs, from $2.76 \times 10^{-3} \mathrm{~cm} / \mathrm{s}$ for $13-\mathrm{nm}$ AuNPs to $1.77 \times 10^{-3} \mathrm{~cm} / \mathrm{s}$ for $75-\mathrm{nm}$ AuNPs (Figure 16). This might be ascribed to the failure of the larger AuNPs to fill the interstitial spaces between the carbon nanotube networks. 

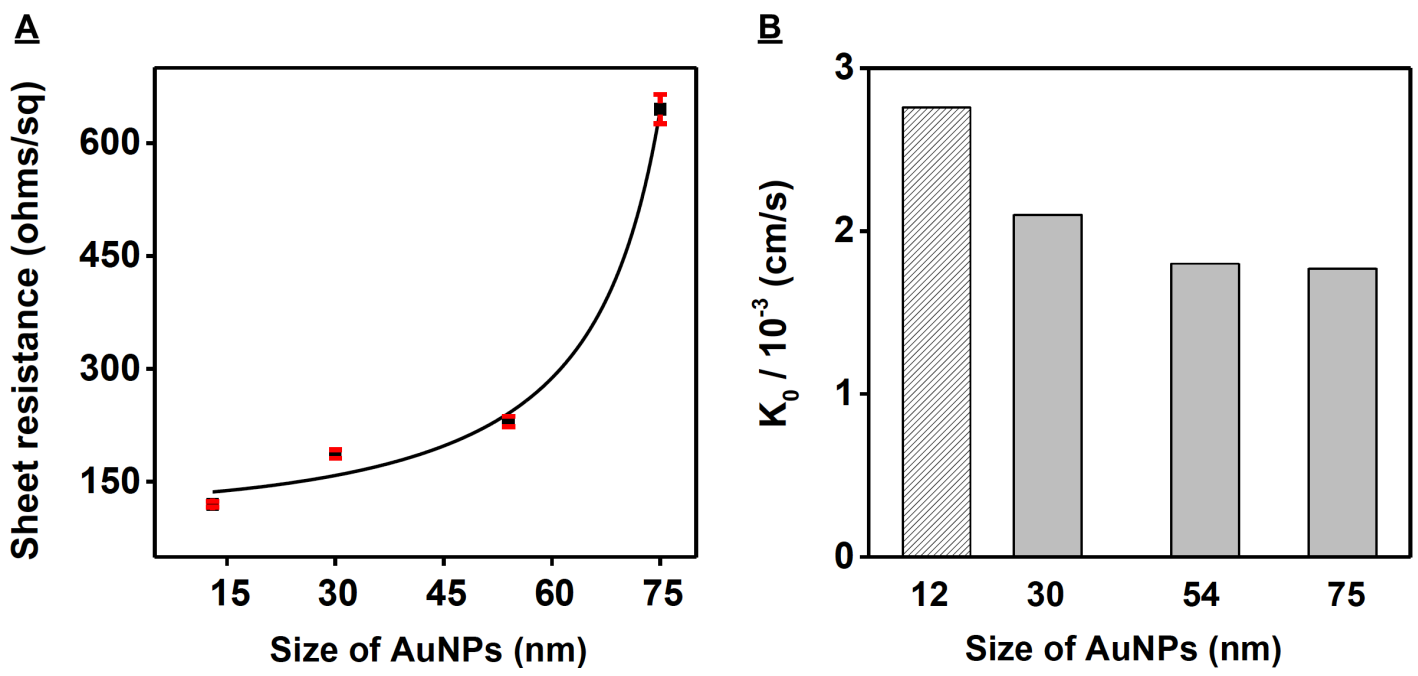

Figure 16: Effect of AuNP size on sheet resistance and electron transfer rates. (A) Sheet resistance comparison of VF-fabricated gold films prepared from AuNPs with diameters of 12, 30, 54, and $75 \mathrm{~nm}$. (B) Electron transfer rate comparison of the same films.

To better understand how the structural features of these films contribute to their electrical properties, we characterized the morphology of MCE filter paper and various other films using atomic force microscopy (AFM). AFM is fundamental for studying surface roughness at the nanoscale. We used the average roughness (Ra), which is the mean height as calculated over the entire measured area, to detect general variations in overall profile height characteristics, ${ }^{172}$ where a small $\mathrm{Ra}$ value shows a smooth surface. ${ }^{173} \mathrm{MCE}$ filter paper offers a high degree of the internal surface area for more prominent adsorption of material, and takes into account high stream rate of filtration because of its pores. ${ }^{174,175}$ 
A

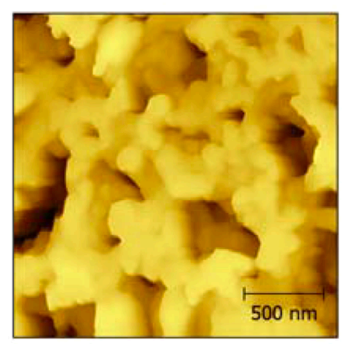

D

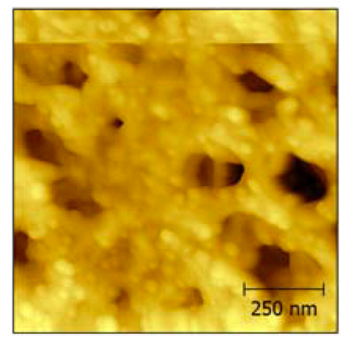

B

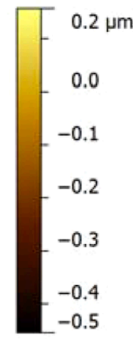

$-0.5$

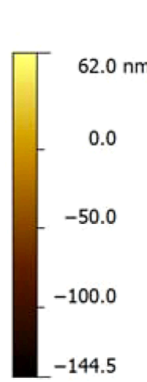

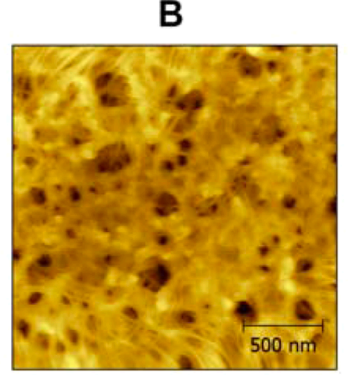

$E$
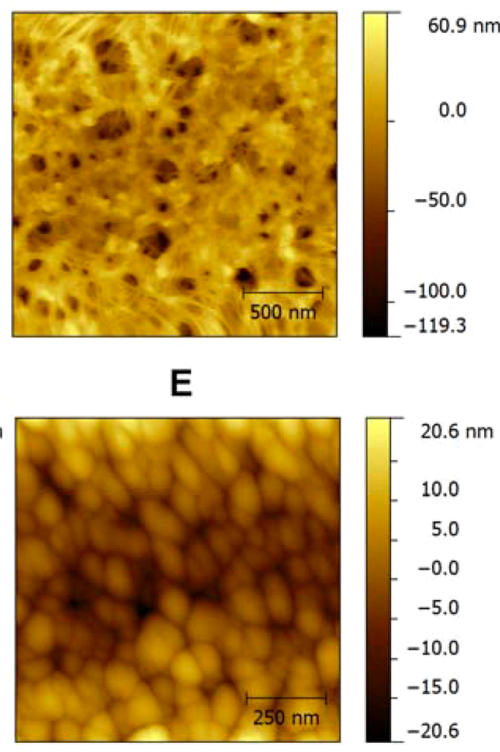

C
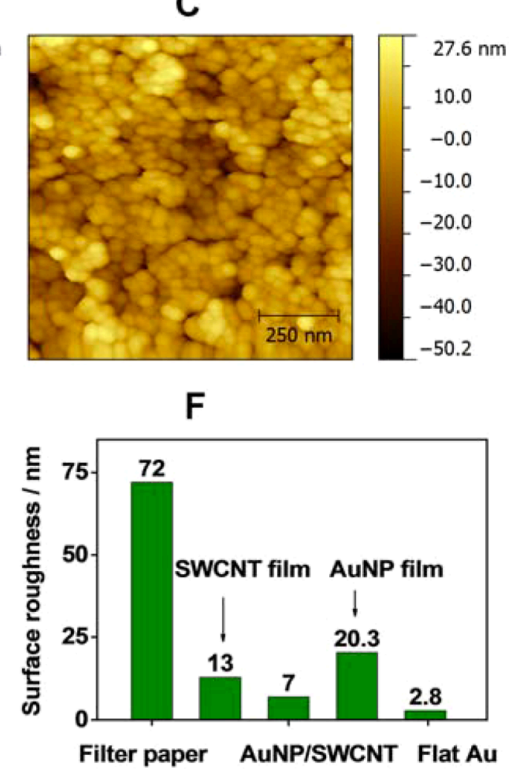

Figure 17: Surface morphology as characterized by atomic force microscopy (AFM). Typical AFM images of (A) MCE filter paper, (B) M-SWCNT-loaded MCE paper, (C) VF-fabricated AuNP/M-SWCNT film, (D) AuNP-loaded MCE paper, and (E) a commercially-purchased gold slide. (F) Surface roughness measurements for the various films shown in A-E. The images were obtained in the tapping mode using a Multimode AFM IIID with Bruker AFM probes (TESP-V2, 0.01-0.025 Ohm-cm Antimony (n) doped $\mathrm{Si})$.

Experimental results demonstrated that MCE paper has a profoundly permeable structure with an interconnected framework, with a mean surface roughness of $72.0 \mathrm{~nm}$ (Figure 17, A and F). When only M-SWCNTs were loaded onto the MCE paper, the resulting network formed an interconnected, hydrophobic surface layer with $13.0 \mathrm{~nm}$ mean surface roughness, thus presenting a six-fold smoother surface (Figure 17, B and F). The subsequent deposition of 35 picomoles of AuNPs onto the M-SWCNT underlay resulted in an even smoother film (Figure 17C), with an average mean surface roughness of 7.0 nm (Figure 17F). Despite the fact that most of the filter channels are sealed with AuNPs, this relatively smooth gold film still retains a porous architecture (Figure 17C). In contrast, the vast majority of the large channels in the MCE filter were open on the AuNP-only film, with small amounts of AuNPs deposited around the pore edges (Figure 
17D). In this scenario, we observed no obvious interconnection/overlap among the AuNPs and a mean surface roughness of $20.3 \mathrm{~nm}$ (Figure 17F). We found that the purchased gold slide was extremely smooth, with a mean surface roughness of $2.8 \mathrm{~nm}$ (Figure 17, E and F).

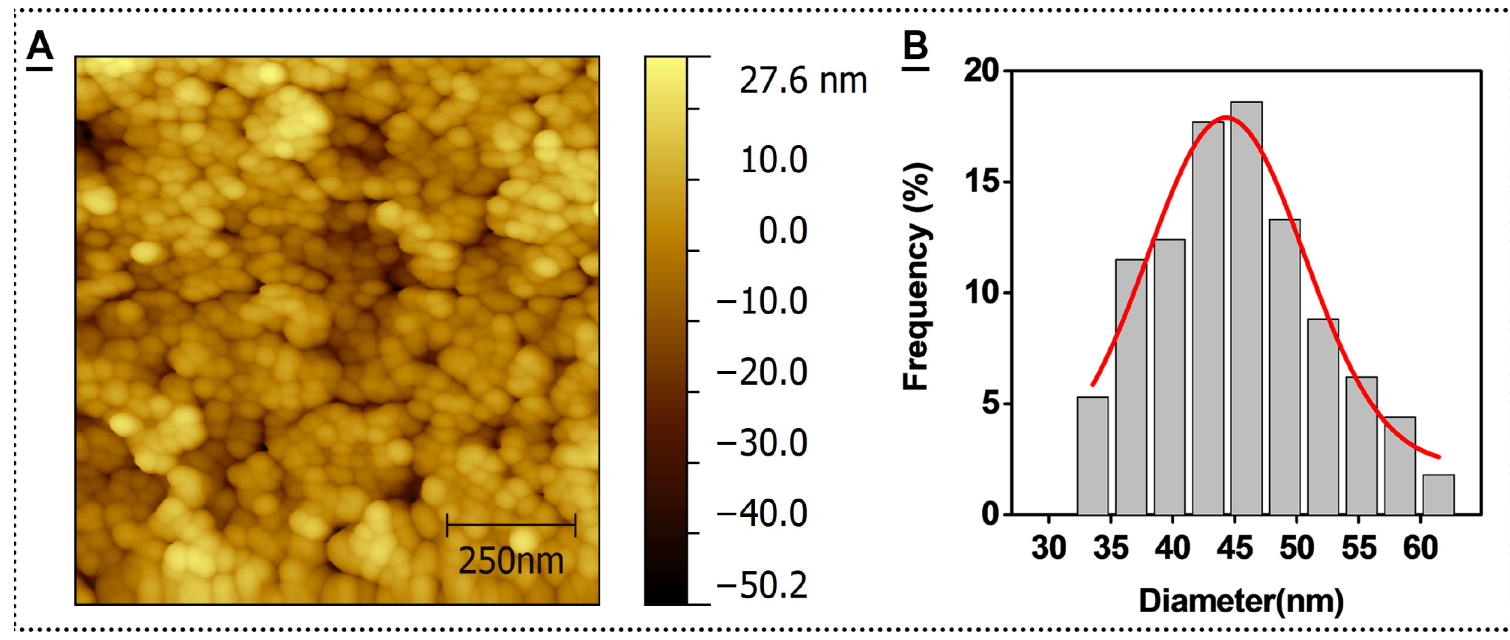

Figure 18: AFM images of (A) the AuNP/M-SWCNT hybrid film prepared with $8 \mu \mathrm{g}$ MSWCNTs and 35 pmole AuNPs. (B) The frequency distribution histogram of the AuNP/M-SWCNT hybrid film shows that the average size of AuNPs is $\sim 45 \mathrm{~nm}$ due to the compact overlapping arrangement of the particles, as calculated using Image $J$ software.

We noted that AuNPs deposited on the M-SWCNT film seem bigger ( $\sim 45 \mathrm{~nm}$ ) (Figure 18, A and B) than the AuNPs $(\sim 13 \mathrm{~nm})$ used for film preparation. This can be explained by the closely-packed nature of the AuNPs on the composite film, which arises from the strong vacuum force creating overlapping particle structures on the M-SWCNT surface. 


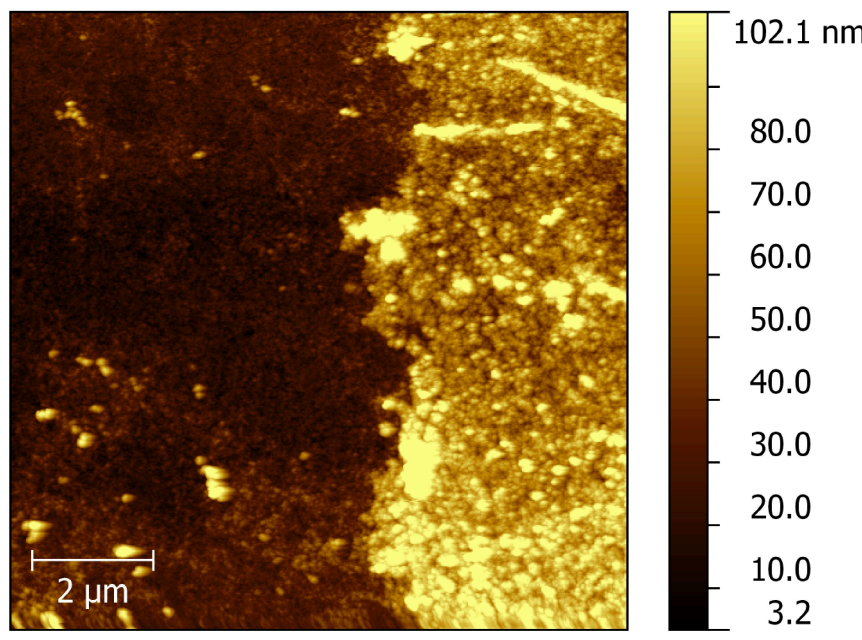

Figure 19: AFM image of the edge of a VF-fabricated gold film prepared with $8 \mu \mathrm{g}$ MSWCNTs and 35 pmole AuNPs. Measurement was made using Nanoscope 3D from Bruker-Nano (Veeco), with a Multimode-Tapping, TESP-V2; 0.01-0.025 Ohm-cm Antimony (n) doped Si AFM probe. The thickness of the edge was measured to be 40 $\mathrm{nm}$.

We then transferred this paper-based AuNP/M-SWCNT hybrid film onto a clean glass substrate using acetone evaporation ${ }^{174}$ and measured the thickness of our hybrid film to be $40 \mathrm{~nm}$ via AFM (Figure 19).

A

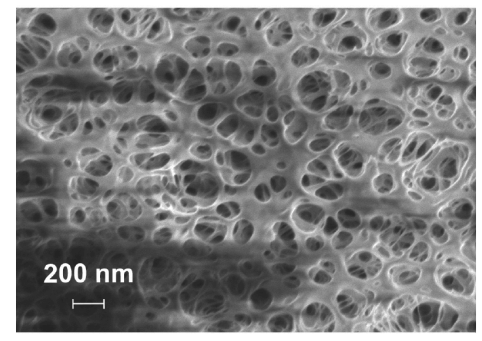

MCE filter paper

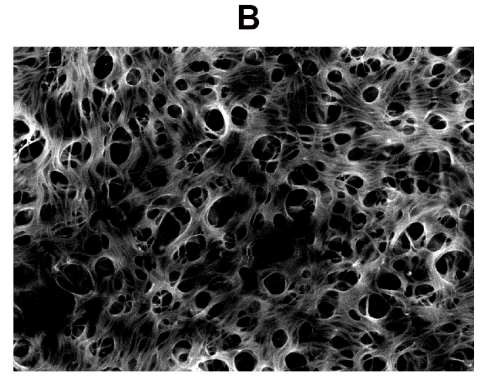

M-SWCNT-loaded MCE paper

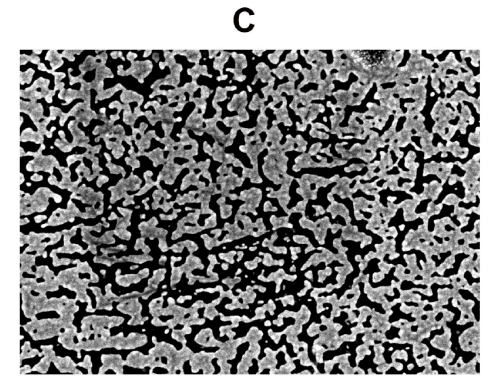

AuNP/M-SWCNT hybrid film

Figure 20: Surface morphology of various films as characterized by SEM. SEM images of (A) MCE filter paper, (B) M-SWCNT-loaded MCE paper, and (C) AuNP/M-SWCNT hybrid film on MCE paper. Scanning electron microscopes (MERLIN, Carl Zeiss, Oberkochen, Germany) were used for the SEM analysis. SEM images of surface of the films were obtained at the accelerating voltage of $3 \mathrm{kV}$.

To further prove the hybrid gold film is permeable, we used scanning electron microscopy (SEM) to characterize various films. The porosity of MCE filter paper was 
also evident from the SEM image (Figure 20A). Deposition of a layer of M-SWCNTs on the paper surface formed an inter-connected conductive network through and over the pores without totally obstructing the pore-space (Figure 20B). Sequential deposition of AuNPs on top of the interconnected network of M-SWCNTs resulted in the formation of a sponge-like, permeable, inter-connected gold film (Figure 20C). Obviously the porosity of the filter paper, with interstitial spaces in between the inter-connected network of AuNPs, remained, and pores and ligaments were interspaced uniformly throughout the surface of the film.

A

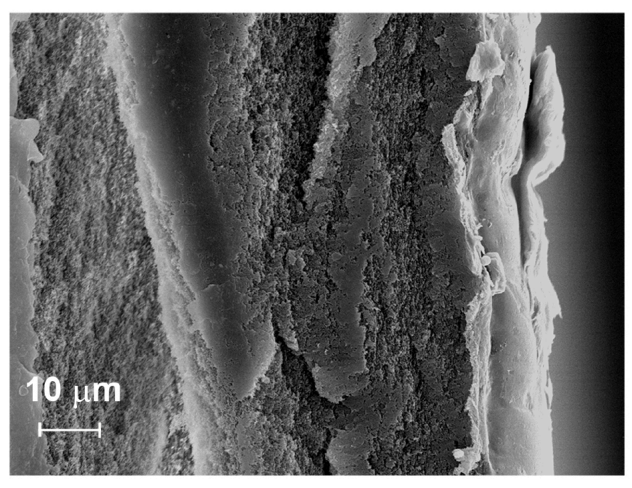

Comprehensive view
B

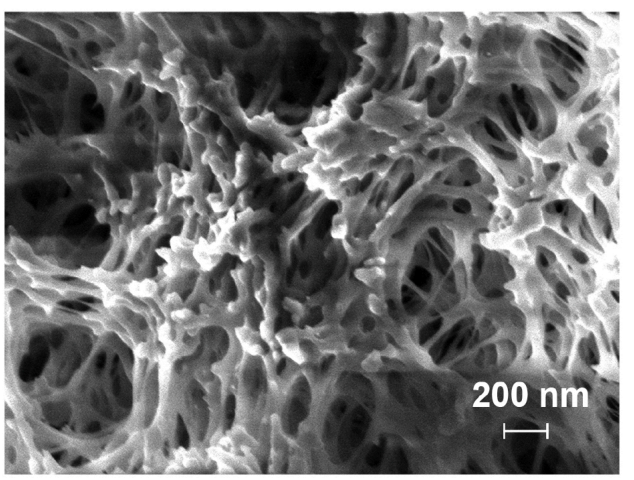

Interior view

Figure 21: Cross-sectional SEM images of our hybrid gold films. (A) A comprehensive view of the cross-section of the paper-based hybrid gold film. (B) An interior view of MCE paper and nanomaterial interfacial layer. Scanning electron microscopes (MERLIN, Carl Zeiss, Oberkochen, Germany) were used for the SEM analysis. SEM images of surface of the films were obtained at the accelerating voltage of $3 \mathrm{kV}$.

Surface SEM images of the hybrid film correlate with the AFM findings, and further corroborate the evidence for the formation of a porous hybrid gold film. We have also performed cross-sectional SEM in order to investigate the infiltration depth of AuNPs within M-SWCNT films. A comprehensive view of the cross-section of the paper-based hybrid gold film can clearly differentiate between the filter paper and nanomaterial layers (Figure 21A). Additionally, an interior view of MCE paper and nanomaterial interfacial 
layer suggested no seepage of AuNPs after its deposition onto the M-SWCNT-loaded paper (Figure 21B).

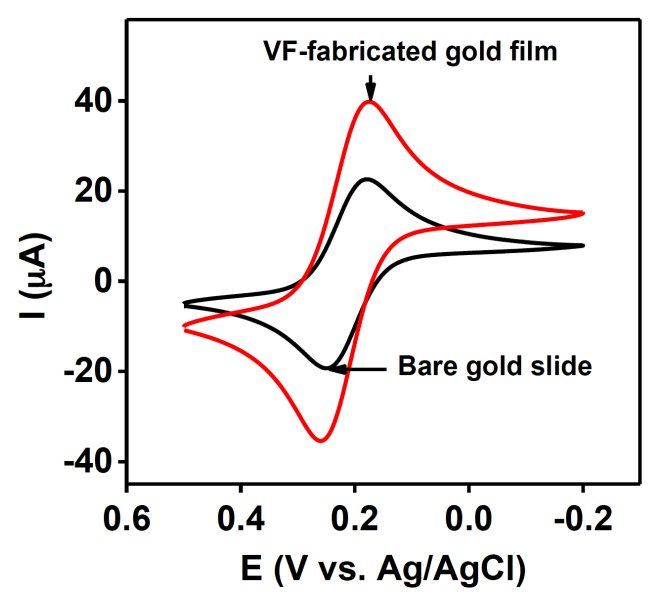

Figure 22: Comparison of the electrochemical characteristics of a flat gold slide relative to our VF-fabricated porous gold film. Cyclic voltammograms were performed in $2 \mathrm{mM}$ $\mathrm{K}_{3} \mathrm{Fe}(\mathrm{CN})_{6}{ }^{3-} / \mathrm{K}_{3} \mathrm{Fe}(\mathrm{CN})_{6}{ }^{4-}$ solution at a scan rate of $10 \mathrm{mV} / \mathrm{s}$.

The VF-fabricated hybrid thin gold films generate a higher current than flat gold slides due to their porosity (Fig. 22). We utilized a single sheet of film to make fourteen working electrodes $(5 \mathrm{~mm} \mathrm{~W} \times 8 \mathrm{~mm} \mathrm{~L})$ and compared the electroactive surface area of these electrodes in $0.5 \mathrm{M} \mathrm{H}_{2} \mathrm{SO}_{4}$ relative to a commercially-purchased flat gold slide with the same geometric surface area. The voltammograms of both gold electrodes demonstrated that oxidation started at $\sim 1.2 \mathrm{~V}$ in the oxidation sweep, generating a broad anodic peak. The formed gold oxide was then electrochemically reduced at $\sim 0.85 \mathrm{~V}$ in the reduction sweep (Figure 23). We calculated the charge transferred by integrating the reduction peak of gold oxide to obtain an electroactive surface area of 0.62 and $0.16 \mathrm{~cm}^{2}$ for the VF-fabricated hybrid gold film and the flat gold slide, respectively, assuming that $386 \mu \mathrm{C} / \mathrm{cm}^{2}$ is required to reduce a monolayer of gold oxide. ${ }^{170}$ This shows that the VFfabricated hybrid thin gold film is 3.2-fold rougher than the flat gold slide. The larger surface area of the VF-fabricated hybrid gold film may be due to a combination of the 
high surface-to-volume ratio of the carbon nanotube support along with the porosity of the paper substrate.

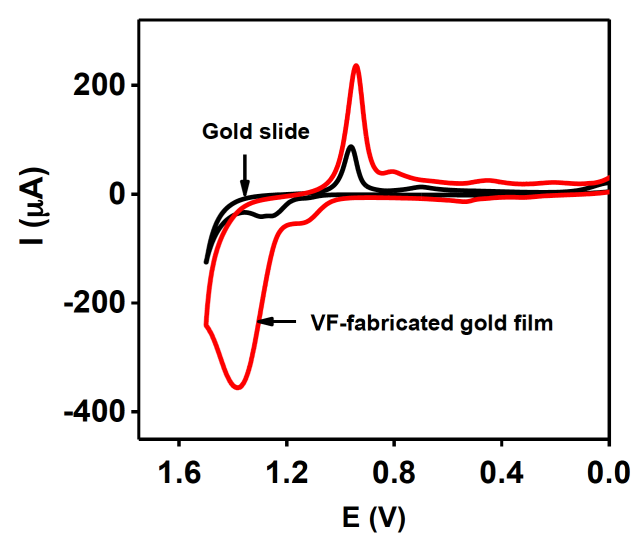

Figure 23: Comparison of the electrochemical characteristics of a flat gold slide relative to our VF-fabricated porous gold film. Cyclic voltammetry was performed in $0.5 \mathrm{M}$ $\mathrm{H}_{2} \mathrm{SO}_{4}$ at a scan rate of $50 \mathrm{mV} / \mathrm{s}$.

The neurotransmitters dopamine (DA) and serotonin (5-HT) were utilized as benchmark analytes to evaluate our film's electrocatalytic properties. We observed excellent electrocatalytic performance for detecting both molecules independently with our hybrid gold films (Figure 24A and C), with only a small background current in the absence of analytes. A solution of $1 \mathrm{mM}$ DA or 5-HT exhibited quasi-reversible electrochemical behavior, with a respective 60-fold (DA, Figure 24A, red trace) and 45-fold (5-HT, Figure $24 \mathrm{C}$, red trace) increase in electrocatalytic oxidation current at $0.19 \mathrm{~V}$ and $0.39 \mathrm{~V}$. In contrast, film comprised entirely of M-SWCNTs produced only a small electrochemical response to DA (Figure 24A, blue trace) or 5-HT (Figure 24C, blue trace) at $0.8 \mathrm{~V}$. Therefore, even though DA may diffuse through the M-SWCNT layer, its electrochemical response is relatively small. We obtained a 3-fold smaller oxidation current for DA at $0.18 \mathrm{~V}$ from a flat gold electrode with the same geometric surface area 
as our VF-fabricated gold film (Figure 24B, black trace), presumably because of its absence of porosity.
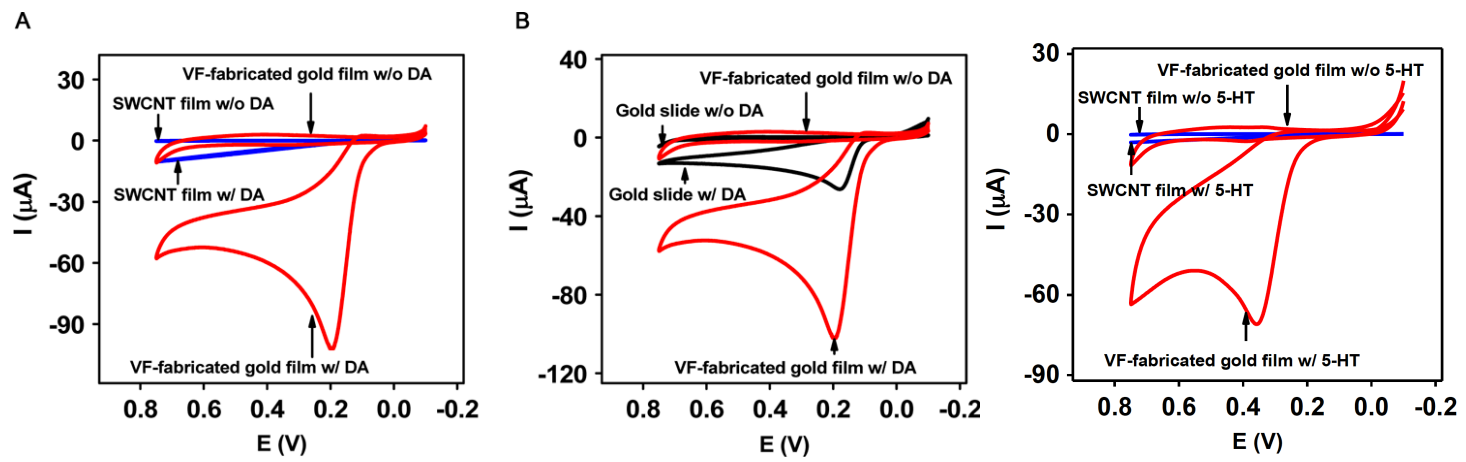

Figure 24: Comparison of the electrochemical responses of our VF-fabricated gold film relative to other films. (A) Cyclic voltammograms of our VF-fabricated gold film and a M-SWCNT-only film in 0.1 M phosphate-buffered saline (PBS, $\mathrm{pH}$ 7.4) with and without $1 \mathrm{mM}$ dopamine (DA). (B) Cyclic voltammograms of our VF-fabricated gold film and a purchased gold slide with the same geometrical dimensions in 0.1 M PBS (pH 7.4) in the absence or presence of $1 \mathrm{mM}$ DA. (C) Electrocatalytic oxidation of 5-HT in $0.1 \mathrm{M}$ PBS $(\mathrm{pH} 7.4)$ with a VF-fabricated gold film electrode with sheet resistance of $126 \mathrm{ohm} / \mathrm{sq}$ (red) versus M-SWCNT-only films with sheet resistance of 75,000 W/sq (blue). 5-HT yields a 45 -fold increase in peak current at $0.39 \mathrm{~V}$ (vs. saturated calomel electrode) with our hybrid film versus film composed only of M-SWCNTs.

The increased active surface area of our VF-fabricated gold film clearly produces a higher turnover for electrochemical reactions at the electrode surface, resulting in large faradic current and sensitive analyte detection. To demonstrate this, we performed electrocatalytic oxidation with different concentrations of DA and 5-HT using VFfabricated hybrid thin gold film electrodes. The electrocatalytic current increased linearly with increasing DA concentrations over a wide range from $50 \mathrm{nM}$ to $1 \mathrm{mM}\left(\mathrm{R}^{2}=0.996\right)$ (Figure 25), with a detection limit of $10 \mathrm{nM}$. 
$\underline{A}$

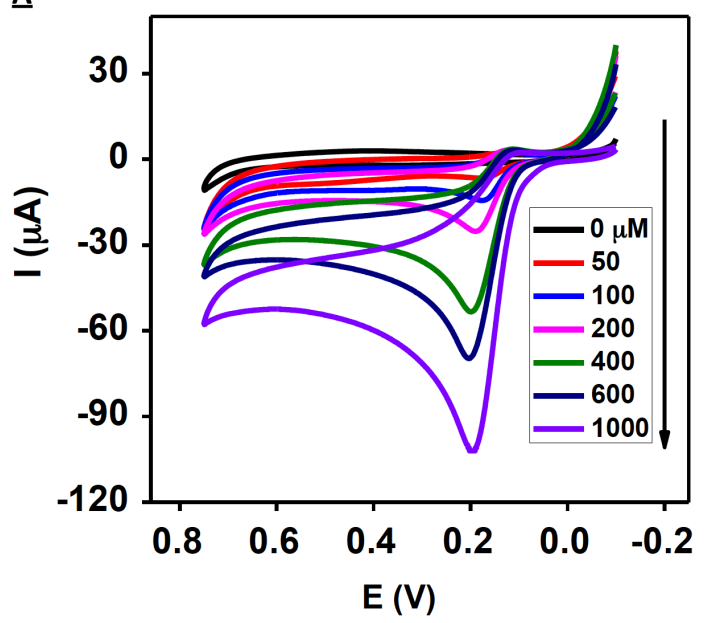

$\underline{B}$

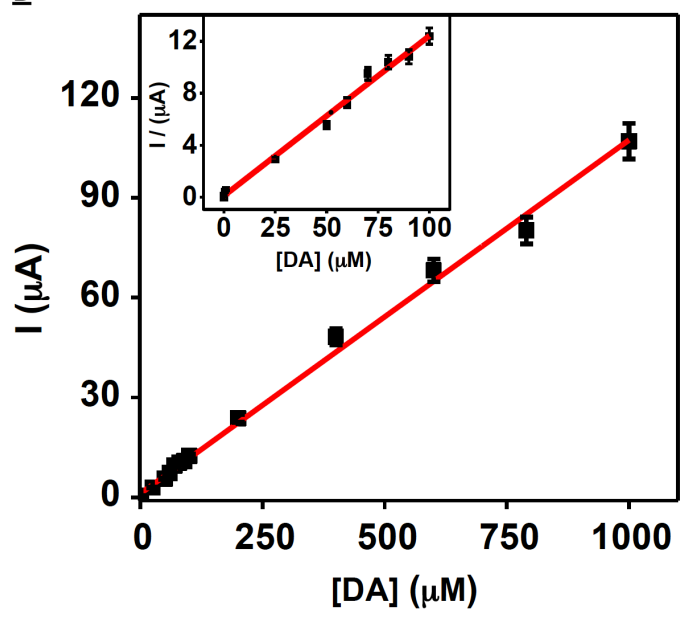

Figure 25: DA is electrocatalytically oxidized on our VF-fabricated porous gold film electrode. (A) Cyclic voltammetric responses of various concentrations of DA in $0.1 \mathrm{M}$ PBS ( $\mathrm{pH}$ 7.4). (B) The anodic current at $0.19 \mathrm{~V}$ increased linearly with increasing DA concentrations in the range of $50 \mathrm{nM}$ to $1 \mathrm{mM}$, with inset showing linearity at concentrations ranging from 0 to $100 \mu \mathrm{M}$. The data reported are averages of three individual experiments.

A similar linear response was observed with 5-HT: the peak current increased with increasing 5-HT concentration, exhibiting a linear relationship from $250 \mathrm{nM}$ to $1 \mathrm{mM}\left(\mathrm{R}^{2}\right.$ $=0.997)$ with a detection limit of $50 \mathrm{nM}$ (Figure 26).

$\underline{\mathbf{A}}$

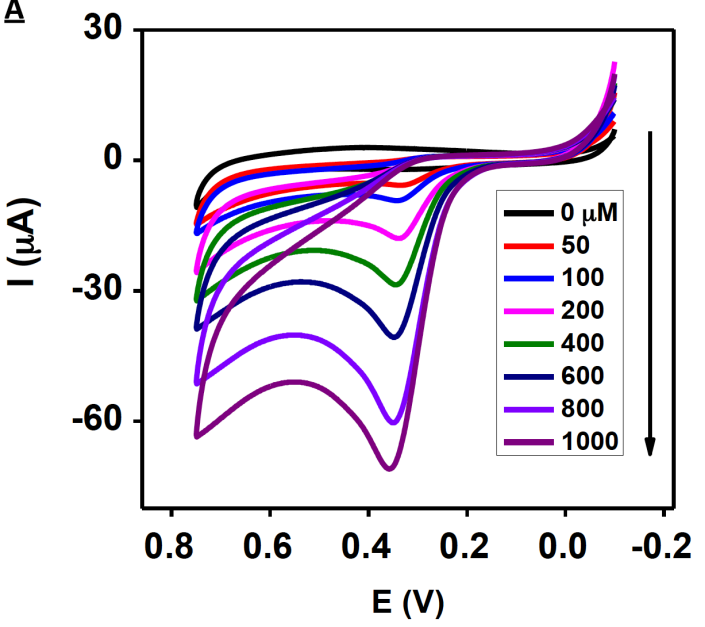

$\underline{B}$

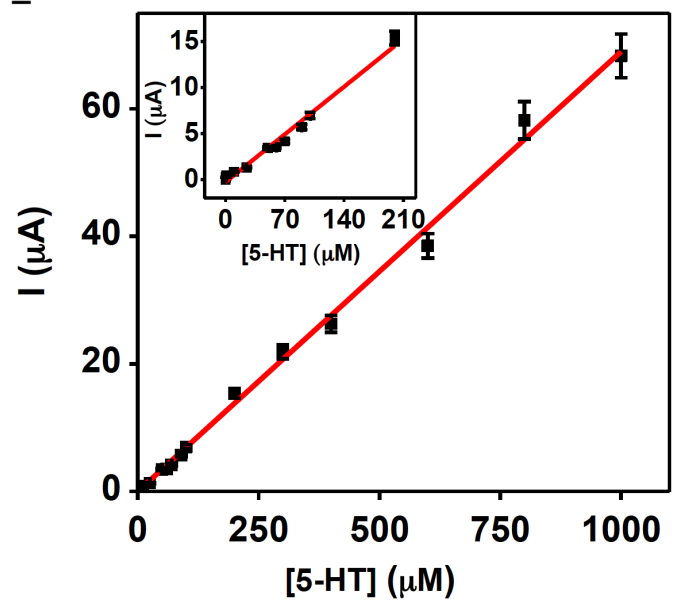

Figure 26: Electrocatalytic oxidation of 5-HT at different concentrations on VFfabricated gold film electrodes. (A) Cyclic voltammetric responses of various concentrations of 5-HT in 0.1 M PBS (pH 7.4). (B) The electrocatalytic anodic current at 
$0.39 \mathrm{~V}$ increased linearly with increasing 5-HT concentrations in the range of $200 \mathrm{nM}$ to $1 \mathrm{mM}$, with inset showing linearity at concentrations ranging from 0 to $200 \mu \mathrm{M}$. The data reported are averages of three individual experiments.

Our hybrid gold films exhibited great reproducibility and storage stability for target detection; we obtained a standard deviation of 3\% after testing 14 VF-fabricated gold film electrodes with DA (Figure 27A), and a standard deviation of $4 \%$ for DA response for electrodes tested every day over a storage period of two weeks (Figure 27B).
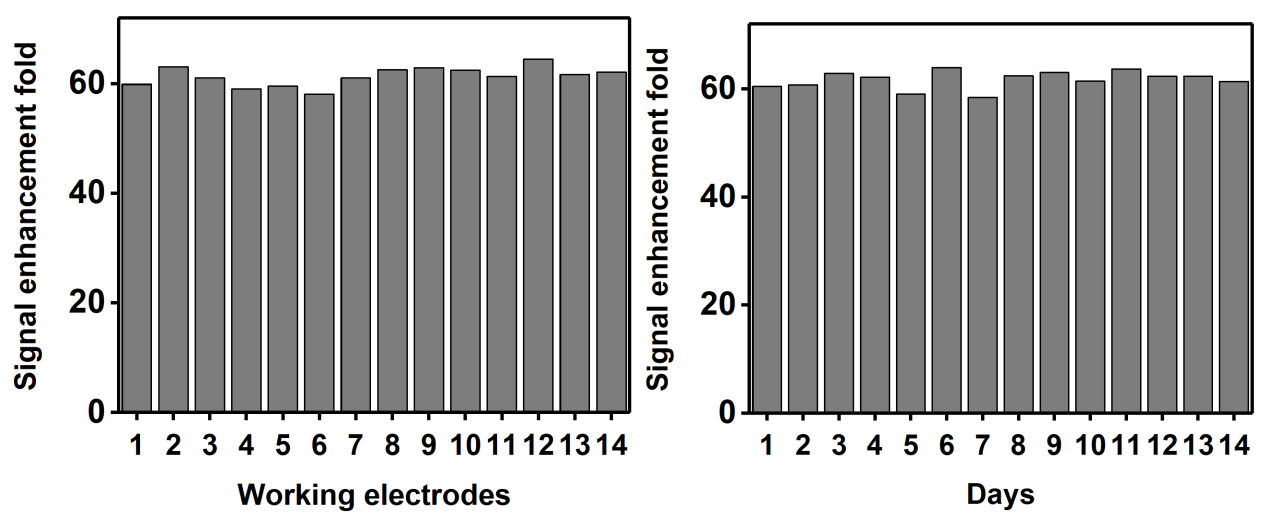

Figure 27: (A) Electrodes constructed from our hybird gold film demonstrated great reproducibility in small molecule detection. Results are from 14 electrodes made from a single piece of hybrid gold film, tested with $1 \mathrm{mM}$ DA in $0.1 \mathrm{M}$ PBS (pH 7.4). (B) Stability of our hybrid gold film electrodes over a period of two weeks. Results are from 14 electrodes made from a single piece of our hybrid gold film, with one electrode tested per day for electrocatalytic response of $1 \mathrm{mM} \mathrm{DA}$ in $0.1 \mathrm{M} \mathrm{PBS}(\mathrm{pH} 7.4)$ over a period of two weeks.

Additionally, surface cleaning of our hybrid gold film electrodes performed in $\mathrm{NaOH}$ and $\mathrm{H}_{2} \mathrm{SO}_{4}$ solution with the electrochemical protocol ${ }^{176}$ demonstrated a mere $1.5 \%$ increase in electron transfer rate, suggesting that these paper-based electrodes are suitable for immediate use (Figure 28). 


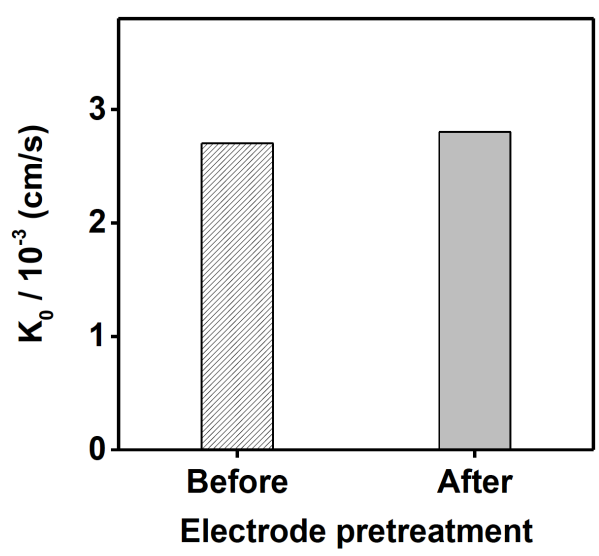

Figure 28: Effect of surface cleaning treatment on our hybrid gold film on electron transfer rate. A $1.5 \%$ increase in electron transfer rate was observed after electrode pretreatment, suggesting that pretreatment is unnecessary. Electron transfer rate measurements were performed in $2 \mathrm{mM} \mathrm{K}_{3} \mathrm{Fe}(\mathrm{CN})_{6}{ }^{3-} / \mathrm{K}_{3} \mathrm{Fe}(\mathrm{CN})_{6}{ }^{4-}$ solution at scan rates of $10,20,50$, and $100 \mathrm{mV} / \mathrm{s}$.

The VF-fabricated gold film electrode also demonstrated good stability during measurement. Since the AuNPs were physically deposited on the surface of the MSWCNTs by vacuum force, we evaluated their stability with a film-soaking test where the as-prepared hybrid film electrodes were pre-soaked in 0.1 M PBS for various periods of time before measuring electron transfer rate. We did not observe any visible stripping of gold film during the test, and obtained only an $8 \%$ decrease in the electron transfer rate after a 45-min soaking (Figure 29). 


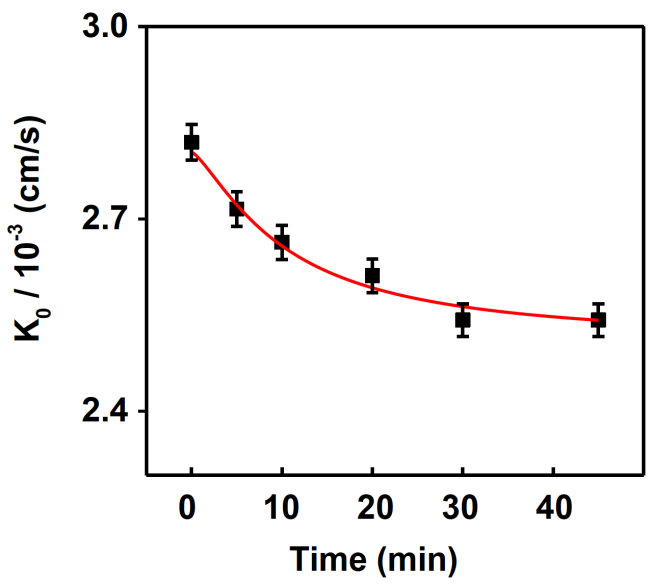

Figure 29: Effect of solution soak time on electron transfer rate for our hybrid gold films. A small decline (8\%) in the electron transfer rate was observed after soaking $45 \mathrm{~min}$ in 0.1 M PBS solution, but with no visible stripping observed on the surface of the film. Measurements were performed in $2 \mathrm{mM} \mathrm{K}_{3} \mathrm{Fe}(\mathrm{CN})_{6}{ }^{3-} / \mathrm{K}_{3} \mathrm{Fe}(\mathrm{CN})_{6}{ }^{4-}$ solution at scan-rates of $10,20,50$, and $100 \mathrm{mV} / \mathrm{s}$.

We have also investigated the effect of solution $\mathrm{pH}$ on the stability of our AuNP/MSWCNT hybrid gold film. Specifically, we performed electron transfer rate measurements on the hybrid gold film after incubating in 0.1 M PBS solutions with different $\mathrm{pH}$ values $(5.0,6.0,7.0,8.0$, and 9.0) for 10 minutes. Our experimental results demonstrated that our film is highly stable across all conditions (Figure 30).

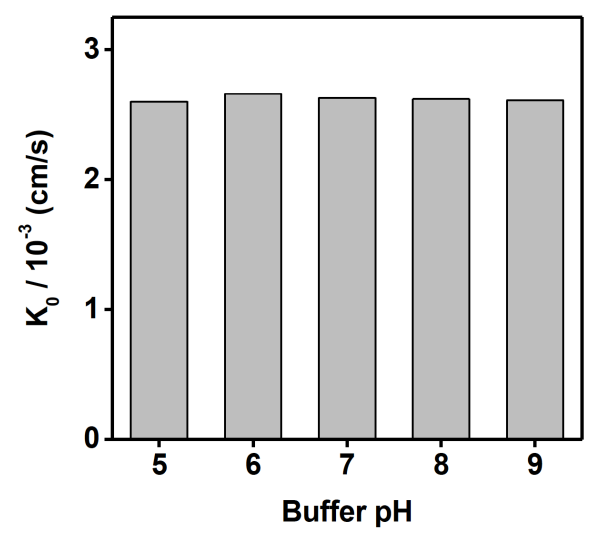

Figure 30: Effect of $\mathrm{pH}$ on electron transfer rate of our hybrid gold film. Electron transfer rate measurements were performed with our hybrid gold film electrode after incubating in $0.1 \mathrm{M}$ PBS solutions with different $\mathrm{pH}$ values $(5.0,6.0,7.0,8.0$, and 9.0) for $10 \mathrm{~min}$. The experimental results demonstrated that our film is highly stable across all tested $\mathrm{pH}$ conditions. 
Additionally, we post-treated the hybrid gold film at 20,50 or $80{ }^{\circ} \mathrm{C}$ for 6 hours to test the effect of temperature on electron transfer rate. We found that hybrid gold films treated at 50 and $80{ }^{\circ} \mathrm{C}$ showed a $8 \%$ and $14 \%$ respective decrease in electron transfer rate compared to the films left at $20{ }^{\circ} \mathrm{C}$ (Figure 31). This decrease is possibly due to a morphological change in the hybrid composite layers, which may have caused separations or breaks in the conductive network at higher temperatures.

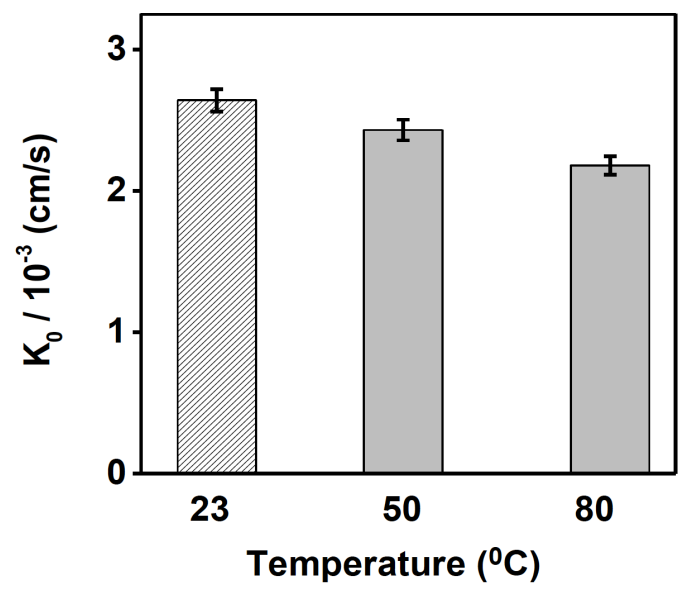

Figure 31: Effect of temperature on electron transfer rate of our hybrid gold film. An 8\% and $14 \%$ decrease in electron transfer rate were observed at elevated temperatures of 50 ${ }^{\circ} \mathrm{C}$ and $80{ }^{\circ} \mathrm{C}$, respectively. This is possibly due to deformation within the structural integrity of the hybrid film. Measurements were performed in $2 \mathrm{mM} \mathrm{K}_{3} \mathrm{Fe}(\mathrm{CN})_{6}{ }^{3-}$ $/ \mathrm{K}_{3} \mathrm{Fe}(\mathrm{CN})_{6}{ }^{4-}$ solution at scan rates of $10,20,50$, and $100 \mathrm{mV} / \mathrm{s}$.

We observed equally robust electrocatalytic responses for DA or 5-HT with our VFfabricated hybrid thin gold film when compared against similar hybrid gold films prepared by electrodeposition methods. We used template-free (TF) ${ }^{169}$ and hydrogen bubble (HB) directed ${ }^{46}$ electrodeposition to produce AuNP films atop MCE paper coated with $8 \mu \mathrm{g}$ of M-SWCNTs. Experimental results demonstrated that both techniques yielded non-uniform, sparsely gold-deposited films, and this limited AuNP deposition resulted in a poor electrocatalytic response to DA oxidation at $0.5 \mathrm{~V}$, yielding a broad 
peak with an 8 -fold lower electrocatalytic current relative to the signal obtained from our VF-fabricated hybrid gold film at $0.19 \mathrm{~V}$ (Figure 32A).

$\underline{A}$

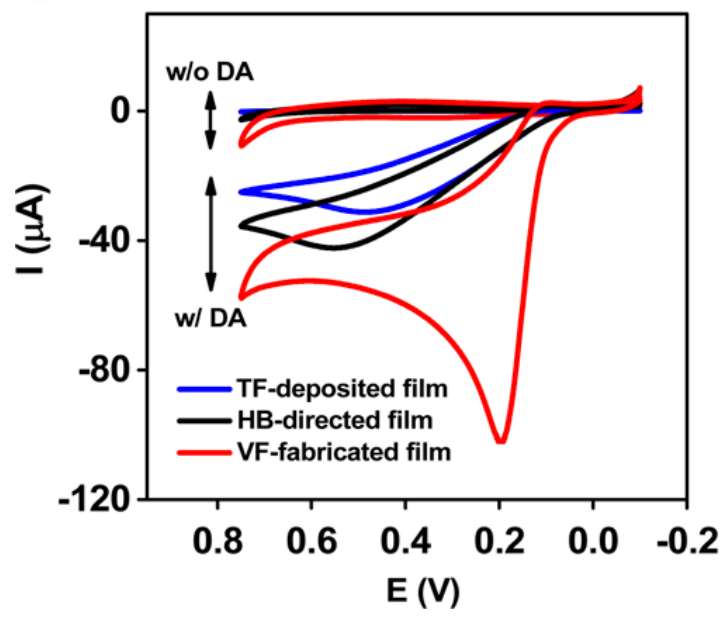

$\underline{B}$

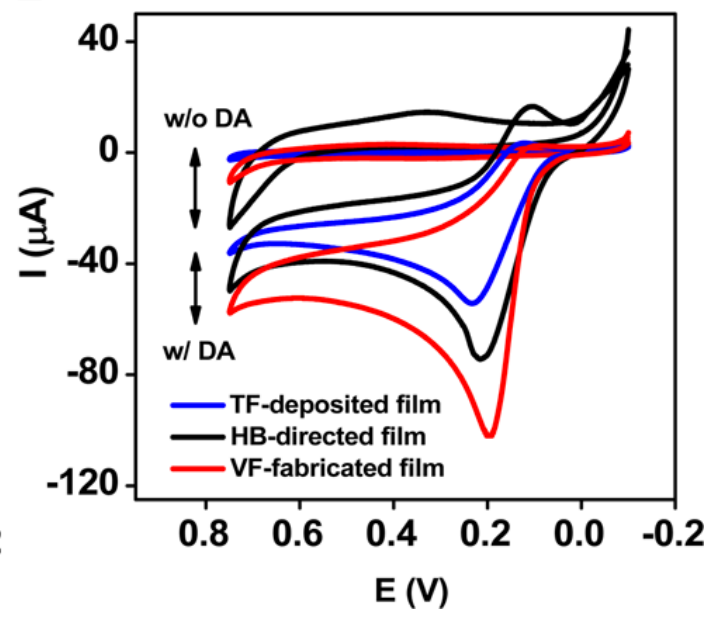

Figure 32: Comparison of electrocatalytic response of DA on various paper-based gold films. (A) We tested gold films fabricated on an underlayer of $8 \mu \mathrm{g}$ M-SWCNTs using vacuum filtration (VF), template-free (TF) electrodeposition and hydrogen bubble (HB)directed electrodeposition. (B) Electrocatalytic response of DA on gold films fabricated on a $40 \mu \mathrm{g}$ underlayer of M-SWCNTs using TF- and HB-directed electrodeposition versus a VF-fabricated gold film atop an $8 \mu \mathrm{g}$ layer of M-SWCNTs.

Indeed, we were unable to use $8 \mu \mathrm{g}$ of M-SWCNTs as a conductive underlayer to electrochemically produce a highly conductive gold film, even after prolonged deposition times $(\sim 8 \mathrm{~h})$. The electrodeposition was hindered by the poorly conductive underlayer formed by $8 \mu \mathrm{g}$ M-SWCNTs $(75,000 \Omega / \mathrm{sq})$. We therefore prepared a filter paper loaded with $40 \mu \mathrm{g}$ of M-SWCNTs, which boasts 15 -fold improved conductivity (5000 $\Omega / \mathrm{sq})$, and this provided a more robust foundation for the electrodeposition of AuNPs. In comparison with the shiny, yellow gold film produced by vacuum filtration, films generated by TF-directed electrodeposition showed a smooth, shiny brownish-red surface, while HB-directed deposition resulted in a rough and flaky dark brown surface. This TF-deposited gold film generated a great response for DA oxidation (Figure 32B), 
with a 65 -fold increase over background - comparable to the 60 -fold increase observed with our VF-fabricated hybrid thin gold film. The low background current obtained with the TF-deposited gold film indicates the lack of porosity in this film. On the other hand, the porous, flaky gold film prepared by HB-directed electrodeposition showed only a 30fold increase in electrocatalytic signal from DA, as the increased porosity resulted in a high background current (Figure 32B). Although TF-deposited gold film achieved a slight higher electrocatalytic response, considerably more time is required to prepare the films ( $\sim 3$ hours per electrode) in comparison with our VF-fabricated gold film $(\sim 20$ minutes for 16 electrodes). Furthermore, at least a five-fold greater amount of MSWCNTs must be loaded onto the paper to produce conductive gold films with excellent electrocatalytic response, which considerably increases the cost of materials.
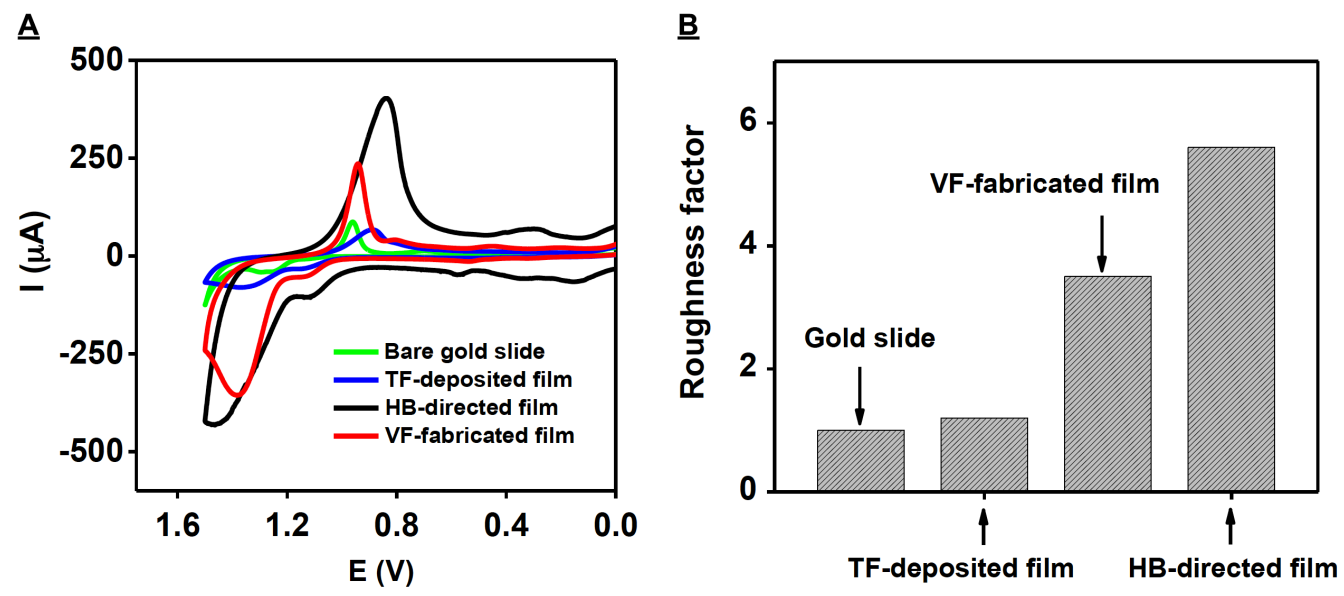

Figure 33: Comparison of electrochemical characteristics and roughness factors of bare, flat gold slides, TF-deposited gold film, HB-directed gold film and VF-fabricated gold film. (A) Cyclic voltammograms of different electrodes in $0.5 \mathrm{M} \mathrm{H}_{2} \mathrm{SO}_{4}$ at a scan rate of $50 \mathrm{mV} / \mathrm{s}$. (B) The calculated roughness factors for our various films.

The difference in porosity among films greatly affects their capacity to discriminate mixtures of analytes. Simultaneous detection of DA and 5-HT is generally difficult due to their similar oxidation potentials and overlapping signals at most solid electrodes. ${ }^{44}$ 
However, it has been demonstrated that porous films have a greater capacity for simultaneously distinguishing multiple analytes. ${ }^{177-180}$ Therefore, we examined the simultaneous detection of DA and 5-HT with VF-fabricated gold films relative to other smooth or porous gold films. We observed that smooth surfaces, such as purchased flat gold slides and TF-deposited gold films (roughness factors of 0.80 and 1.2, respectively; Figure 33) exhibited two broad, overlapping peaks at $0.18 \mathrm{~V}$ and $0.38 \mathrm{~V}$, corresponding to the oxidation of DA and 5-HT, respectively (Figure 34A).
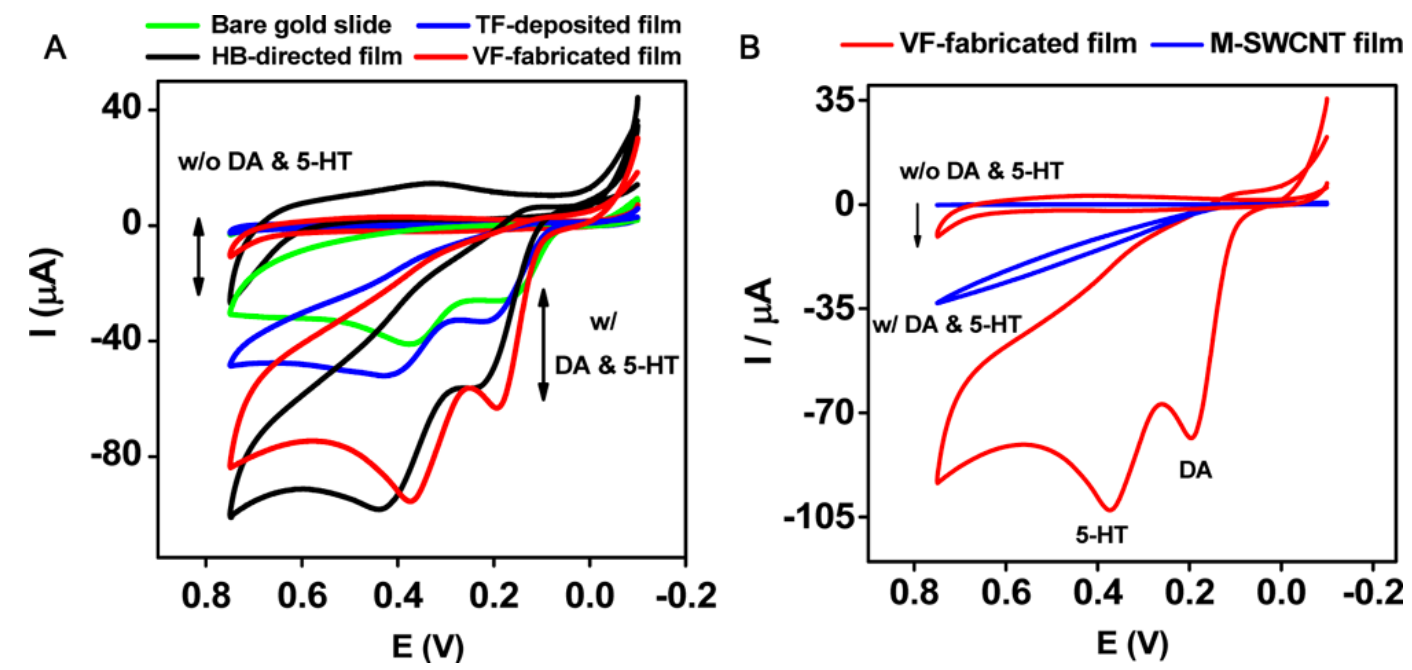

Figure 34: Comparison of simultaneous target detection with various gold films. (A) Porous gold films prepared by HB-directed electrodeposition or VF-fabrication enable improved simultaneous detection of mixtures of DA and 5-HT compared to smooth surfaces such as TF-deposited gold films or purchased gold slides. TF-deposited and HBdirected gold films were prepared on MCE filter paper loaded with $40 \mu \mathrm{g}$ of $\mathrm{M}$ SWCNTs, whereas the VF-fabricated gold film was prepared with an underlayer of $8 \mu \mathrm{g}$ of M-SWCNTs. (B) Simultaneous detection of a mixture of DA and 5-HT on MSWCNT-only or VF-fabricated gold film. Both films were prepared with $8 \mu \mathrm{g}$ of MSWCNTs.

These broad peaks were respectively resolved into two well-defined peaks at $0.19 \mathrm{~V}$ and $0.39 \mathrm{~V}$ on porous gold film electrodes such as the VF-fabricated and HB-directed gold films (Figure 34A) (roughness factors of 3.5 and 5.6, respectively; Figure 33). However, the HB-directed film produced excessively high background current due to its loosely 
bound structure. We observed the best discrimination on our VF-fabricated thin gold film, with a separation of $200 \mathrm{mV}$ between the two sharp peaks-large enough to accurately distinguish the presence of DA and 5-HT simultaneously. In contrast, control experiments demonstrated that an M-SWCNT-only film was incapable of resolving these two oxidation peaks due to its poor conductivity (Figure 34B). The porous nature of the VF-fabricated hybrid gold film electrode, with its highly dispersed catalytic accessibility sites, makes it possible to achieve discriminative current amplification for species with slow reaction kinetics. ${ }^{181}$ Compared with the electrocatalytic current of 5-HT alone, we observed discriminative amplification when mixtures of DA and 5-HT were competitively oxidized on the same VF-fabricated gold surface. For example, we obtained a 45-fold increase in oxidation current from $1 \mathrm{mM}$ 5-HT alone, while a mixture of DA/5-HT yielded a 60 -fold increase in the oxidation current of 5-HT. However, we did not observe meaningful discriminative amplification for DA oxidation, with a 60 -fold increase for DA alone versus a 65 -fold increase for DA in a DA/5-HT mixture. We hypothesize that the pores in our VF-fabricated gold film may trap small volumes of solution even in the deeper regions of the film, making it possible to distinguish rates of electron transfer for multiple species due to negligible mass transfer and shorter diffusion time for molecules residing within the pores. ${ }^{181}$ Because DA has faster electron transfer kinetics than 5-HT, ${ }^{182-184}$ most DA molecules are oxidized completely at the outskirts of the pores before they are able to diffuse into the pores, and the effect of the pore-created inner surface on signal output is therefore greatly reduced. ${ }^{178}$ In contrast, the large active surface area of our porous gold film is favorable for detecting the slower electron transfer from 5-HT due to the confinement of 5-HT molecules within the pores, ${ }^{182}$ generating a 
higher amplification of 5-HT oxidation relative to DA from mixtures of the two molecules.

\subsection{Conclusions}

We report here a simple and new approach for preparing dispensable, paper-based, porous AuNP/M-SWCNT hybrid thin gold films with high conductivity, rapid electron transfer rates, and excellent electrocatalytic properties. Our film preparation process takes $\sim 20$ minutes and requires only two steps of ambient vacuum filtration with two aqueous solutions containing M-SWCNTs and AuNPs, respectively. We show that the MSWCNTs form a porous network of interconnected ligaments and pores, atop which the citrate-coated AuNPs gather into a conductive and porous thin gold film. Note that our VF-fabricated gold films can be prepared onto various types of filter papers, with different sizes of AuNPs or with different types of SWCNTs in a highly reproducible fashion without the requirement for sophisticated instruments or a clean-room environment. To our knowledge, this is the first report on using simple filtration with layer-by-layer deposition to rapidly manufacture such highly conductive gold films in a controlled manner under ambient conditions, with no post-growth, cleaning or sintering steps required after the initial two-stage fabrication. Importantly, the thickness and other properties of our hybrid thin gold film can be easily controlled by varying the concentrations or volumes of the two film preparation solutions. VF-fabricated gold films can distinguish the presence of DA and 5-HT either individually or simultaneously, with a resolution that greatly exceeds that of purchased flat gold slides or porous gold films prepared by more labor-intensive electrodeposition techniques. 
Nanomaterials such as carbon nanotubes or graphene can be utilized as a conductive underlay for the deposition of a variety of different nanoparticles, including gold, silver, copper, platinum or palladium to form various pure or alternating layers of metal films. Such hybrid metal films could be used in electrochemical catalysis or electro-optical devices, as well as in reflective, conductive or energy-collecting metallic coatings. We believe that our technique gives a simple, adaptable and general means for the rapid fabrication of such diverse metal films onto membranes under ambient conditions, and that this process could likewise serve as a prelude to the transfer of such films to other metal or flexible substrates. 


\section{CHAPTER 4: Paper-Based SWCNT Thin Film Electrode for Drug Detection and Disease Diagnosis - Electrocatalytic Detection of NADH in Complex Matrices}

\subsection{Introduction}

Nicotinamide adenine dinucleotide (reduced) is a coenzyme and a ubiquitous biomolecule found in all living cells, and it plays a significant role in the process of electron transfer, and several metabolic reactions. ${ }^{71-73}$ In particular, intracellular NADH serves as a biomarker for several physiological and pathological events. ${ }^{74-77}$ Fluctuations in the levels of NADH in the cells and tissues can correlate to the intracellular redox status, ${ }^{78}$ apoptosis, ${ }^{78}$ neurodegenerative diseases, ${ }^{73,} 75,76$ and mitochondrial anomalies associated with cancer. ${ }^{79}$ The conversion of NADH in hyperplasia cancer cells was higher compared to the normal cells. ${ }^{79}$ To stimulate the biosynthesis of dopamine and tyrosine hydroxylase by NADH makes it an effective supplement in the treatment of parkinson's disease. ${ }^{80}$ An example of another neurodegenerative disorder is Friedreich's ataxia $(\text { FRDA })^{185}$. Friedrich's ataxia is an autosomal recessive degenerative disorder generally characterized by the loss of deep tendon reflexes, progressive limb ataxia, and skeletal abnormalities typically seen before 25 years of age. ${ }^{185}$ The disease is generally caused by a deficiency of mitochondrial protein frataxin that results from decreased transcription of the FRDA gene. ${ }^{185}$ Energetic stress was caused in the frataxin deficient mitochondria because of compromised cellular respiration. ${ }^{185}$ Irregularities in the function of cellular respiration is known to alter the mitochondrial and cellular redox states $\left(\mathrm{NAD}^{+} / \mathrm{NADH}\right)$ via impaired oxidation leading to the accumulation of NADH generated by the TCA cycle. ${ }^{185}$ In vivo studies in mice have shown that frataxin deficient mitochondrial NADH levels are at least 95 fold higher in comparison to wild-type 
mitochondria, consistent with studies done in humans and with other animal models. ${ }^{186}$ Therefore it is quite logical to understand the levels of NADH in the cell extract for a normal and FRDA patient. Generally, intracellular NADH levels were determined by enzymatic analysis, ${ }^{187-191}$ fluorometric analysis, ${ }^{192-194}$ fluorescence micro-imaging techniques, ${ }^{195}$ laser scanning confocal microphotographics, ${ }^{196}$ and two photon excitation micrographics. $^{197}$

Enzymatic assay methods are some of the most widely used methods in determination of levels of intracellular NADH because it participates in more than 300 kinds of enzymatic oxidation/reduction reactions in vivo. ${ }^{198,199}$ The quantification is usually done in two steps with the first step being the extraction step followed by quantification. ${ }^{84}$ The extraction can either be a separate extraction method where NADH is extracted in an alkaline solution to decompose $\mathrm{NAD}^{83}$ or a single extraction where both NAD and NADH are extracted using neutral or mild basic solutions. ${ }^{84}$ Nicotinamide adenine dinucleotide (reduced) was then quantified using spectrophotometric techniques. ${ }^{84}$ An example of such method for the estimation of free NADH in rat liver was demonstrated by measuring the concentrations of substrates of dehydrogenases ( $\beta$-hydroxybutyrate and glutamate-dehydrogenase systems), which are considered to be in equilibrium with the bound form. ${ }^{81}$ Another example of an enzymatic assay utilized the role of NADH as electron carrier in metabolic reactions where cytosolic and plasma membrane oxidoreductases oxidizes cytosolic NADH and NADPH and passes electrons to extracellular targets. $^{82}$

Fluorometric methods are another techniques that utilize the fluorescence change of $\mathrm{NADH}$. It is known that NADH emits strong fluorescence at $460 \mathrm{~nm}$ when excited at 340 
$\mathrm{nm}$, which is not present for NAD. ${ }^{86}$ The fluorometric assay for NADH is simple, however, it is plagued by strong background fluorescence as the fluorescence of NADH is relatively weak in comparison in living cells. Usually, physical cell disruption methods such as ultrasonication, ${ }^{87}$ high-pressure homogenization, ${ }^{88}$ high-speed bead mill, ${ }^{89}$ and chemical methods such as chemical permeation ${ }^{89}$ and enzymatic lysis ${ }^{89}$ are generally used for extraction of NADH for analysis. The extraction of NADH from yeast cells by snailase lysing ${ }^{90}$ and SDS permeation ${ }^{91}$ clearly depicts the levels of intracellular NADH when the fluorescence spectra were compared against the standard NADH solution. ${ }^{91}$ Indirect fluorescence methods such as resazurin to resafurin reduction by NADH in the presence of diaphorase works as an indicator for the level of intacellular NADH. ${ }^{92}$ Similarly amplex red can also be used with the similar mechanism to detect NADH. ${ }^{93}$ Patterson found a linear relationship between NADH level in cytoplasm and mitochondria and the fluorescence intensity in glucose metabolism using a two-photon excitation microscopy. ${ }^{200}$ These techniques help in monitoring the NADH concentrations in live tissue by using near-infrared excitation light that can also excite fluorescent dyes. Background fluorescence can be strongly suppressed as a result of multi-photon absorption in these techniques, however requires highly controlled conditions and expensive light sources for the imaging.

$\mathrm{NADH}$ also has many in vitro applications. One of the in vitro applications is the cocaine metabolite assay developed by Syva to detect benzoylecgonine in urine samples. ${ }^{94}$ The competitive immunoassay strategy (EMIT) causes the change of reaction kinetics for NADH production depending on the presence or absence of the cocaine metabolite. ${ }^{94}$ The drug in the biosample competes with the drug-labeled enzyme for the limited binding 
sites on the antibody. The conjugated-antibody reduces enzyme activity. Depending on the concentration of drug in the biosample that binds to the antibody sites, leaving the enzyme-labeled drug unbound in the solution to freely breakdown G-6-P resulting in the release of single hydrogen ion $\left(\mathrm{H}^{+}\right)$. NAD present in the reagents reacts with the released hydrogen ion to form NADH. The amount of NADH produced during the EMIT reaction is directly proportional to the amount of drug present in the sample that can be detected using ultra-violet spectrophotometery. ${ }^{94}$ The EMIT strategy has been used for urine samples. However, spectroscopic methods are at a disadvantage when working with complex samples because of spectral interferences arising from the inherent presence of turbidity, and colored pigments resulting from biological processes.

Electrochemical methods have been traditionally used to detect many important biological analytes due to its impressive miniaturization of modern microelectronics and the relative paucity of electroactive contaminants in samples. ${ }^{95}$ Nicotinamide adenine dinucleotide (reduced) itself is electrochemically active, and the conventional solid electrodes such as gold, ${ }^{96}$ platinum, ${ }^{97}$ and glassy carbon ${ }^{98}$ have been used as electrodes for NADH oxidation. However, NADH can only be oxidized on these electrodes at potentials above $+1.0 \mathrm{~V} \cdot{ }^{96-98}$ Consequently, various electron mediators such as organic dyes ${ }^{201}$ conducting polymers ${ }^{202}$ and thio-substituted nucleobases ${ }^{203}$ have been employed or modified on the electrode surface to facilitate NADH oxidation. Although the use of such electron mediators seems promising, problems such as defects of surface modification, the relatively cumbersome nature of these assays, lack of long-term electrode stability, low sensitivity and toxicity limit their practical applications. ${ }^{201-203}$ Moreover, the adsorption of $\mathrm{NADH}$ and $\mathrm{NAD}^{+}$on the electrode leads to surface fouling 
resulting in poor sensitivity, stability, and reproducibility. ${ }^{204}$ Therefore it is essential to find a material that will accelerate the process of interfacial electron transfer between NADH and the electrode surface.

The use of carbon nanomaterial electrodes came into light especially carbon nanotubes is of great interest because of their unique structural and electrical properties. ${ }^{205}$ Carbon nanotubes (CNTs) have large activated surfaces and edge-plane sites (defects) that are ideal for performing NADH oxidation at low potentials without any help of redox mediators minimizing surface fouling. ${ }^{206}$ Apart from the edge plane sites the CNTs also possess a three-dimensional electron-conductive network, which amplifies the response for NADH oxidation. To date, several different types of CNT films have been prepared and used for sensing application. The perpetual demand for rapid, inexpensive, and disposable sensing platforms in industrial and clinical settings led to the evolution of paper-based substrates. ${ }^{57}$ Combining the electrocatalytic advantages of CNTs with the non-expensive, disposable features of paper-based substrates, we were able to fabricate a thin CNT film on paper with a simple vacuum filtration technique. We further utilized the porous paper-based CNT film to directly detect NADH in cell-lysate, which serves as a biomarker for several disease conditions, and also act as a prelude to the general application of detecting NADH generated from enzymatic immunoassays for drug screening.

\subsection{Materials and methods}

4.2.1 Chemicals PureTubes Single-Walled Carbon Nanotubes (P-SWCNTs) solution $(0.25 \mathrm{mg} / \mathrm{mL})$ was purchased from Nanointegris. Beta-Nicotinamide Adenine Dinucleotide Disodium Salt Hydrate (NADH) was purchased from Acros Organics. 
Sodium Dodecyl Sulphate (SDS), phosphate-buffered saline (10× PBS, pH 7.4), sulfuric acid (95-98\%), nitric acid (70\%), hydrochloric acid (36.5-38\%), ethanol (99.5\%), acetone (99.9\%), and potassium hexacyanoferrate (III) were purchased from Sigma-Aldrich and used as received. Mixed Cellulose Ester (MCE) filter papers of 100-nm pore size (47-mm diameter and $105-\mu \mathrm{m}$ thickness) were purchased from Millipore. Fast-drying silver paint was purchased from Ted Pella. EMIT II plus - Cocaine metabolite assay developed by syva (EMIT-Neogen), E. coli cell culture-BL21DE-3 2-DT strain, (Luria-Broth) media with tryptone, yeast extract, sodium chloride, Human lymphoblast cell lines GM02152 (normal individual) and GM16207 (FRDA patient), fetal bovine serum, L-glutamine, Tris-HCl, $\mathrm{KCl}$, EDTA, glycerol, Nonidet P-40, DTT with proteinase inhibitors. All solutions were prepared with distilled water (18.2 M $\Omega \mathrm{cm}-1)$ from a Milli-Q Direct-8 water system unless otherwise specified.

4.2.2 Instrumentation Cyclic voltammetry (CV) and differential pulse voltammetry (DPV) experiments were performed using a CHI760D electrochemical station (CHI Instruments) consisting of $\mathrm{CHI}$ software. The electrochemical cell was assembled with a conventional three-electrode system: paper-based P-SWCNT working electrode $(2 \mathrm{~mm}$ width $\times 4$ mm length), an $\mathrm{Ag} / \mathrm{AgCl}$ reference electrode and a Pt counter electrode. Cyclic voltammetry experiments were performed at a scan rate of $10 \mathrm{mV} / \mathrm{s}$ over the relevant potential range using $1 \times \mathrm{PBS}(\mathrm{pH}$ 7.4), diluted and undiluted pooled saliva, diluted and undiluted E. coli and human normal and FRDA lymphoblast cell extracts. Sheet resistance was measured using a Keithly source meter 4200 with four-probe setup. Electron transfer rate measurements of the paper-based P-SWCNT electrodes were collected using a $\mathrm{K}_{3} \mathrm{Fe}(\mathrm{CN})_{6}{ }^{3-} / \mathrm{K}_{3} \mathrm{Fe}(\mathrm{CN})_{6}{ }^{4-}$ redox probe in aqueous solution. All 
measurements and experiments were carried out at room temperature. TECAN spectrophotometer was used to measure the absorbance of NADH produced from enzymatic reaction at $340 \mathrm{~nm}$. Atomic force microscopy (AFM) was used to measure the thickness of the P-SWCNT film. The AFM topography images of our P-SWCNT film was acquired in the tapping mode using a Multimode AFM IIID (Veeco Instruments) using Bruker AFM probes (TESP-V2; 0.01-0.025 Ohm-cm Antimony (n) doped Si).

4.2.3 Preparation of paper-based P-SWCNT film: The procedure for the fabrication of our paper-based P-SWCNT film is schematically illustrated in Figure 1. A $10 \mathrm{~mL}$ solution containing variable amounts $(12.5,20,25,50$, and $75 \mu \mathrm{g})$ of P-SWCNT solution $(0.25 \mathrm{mg} / \mathrm{mL}$ as stock concentration), was dispersed in $1 \%$ SDS with a Fisher Scientific FS-60D sonication system for 10 min prior to use. The MCE filter paper with $100 \mathrm{~nm}$ pore size was moistened and washed with DI water. The dispersed P-SWCNT solution was added to the moistened filter paper under vacuum conditions at a flow-rate of 0.6 $\mathrm{mL} / \mathrm{minute}$ to form a thin, uniform layer on the surface. This film was washed with DI water to remove the residual SDS surfactant. The film was then left to vacuum dry overnight before using for making electrodes. Working electrodes were prepared by cutting the dried P-SWCNT thin film into rectangular shapes with dimensions of $2 \mathrm{~mm}$ width and $4 \mathrm{~mm}$ in length. Electrical connection was made to the copper wire with quick drying silver paint and wrapped together in place. Thus prepared electrodes are ready to use and require no further modification.

4.2.4 E. coli cell extract preparation $E$. coli cell culture was prepared by inoculation of a loop-full of wild type E. coli BL21DE-3 2-DT strain from a glycerol broth slurry stored at $-80^{\circ} \mathrm{C}$ into $50 \mathrm{~mL}$ of autoclaved L-broth (Luria) media, which was prepared from 10 
$\mathrm{g} / \mathrm{L}$ tryptone, $5 \mathrm{~g} / \mathrm{L}$ yeast extract, and $10 \mathrm{~g} / \mathrm{L} \mathrm{NaCl}$. The inoculated culture was placed in an incubator at $37^{\circ} \mathrm{C}$ with orbital shaking in an Erlenmeyer flask. Cells were harvested at an optical density $\left(\mathrm{OD}_{600}\right)$ reading of 1.62 after a 24-hour growth. The resultant cell suspension was centrifuged for $15 \mathrm{~min}$ at $8500 \times \mathrm{g}$ and $4{ }^{\circ} \mathrm{C}$, pelleted cells were collected and resuspended in $1.62 \mathrm{~mL}$ of $0.1 \mathrm{M}$ phosphate buffer solution $(\mathrm{pH} 7.4)$ to perform the cell-lysis with Misonix Microson XL2000 ultrasonic cell disruptor for 10s pulse and 30s intervals for 4 times in a cold room environment. Ice-water bath was used during the sonication to prevent significant heating in the sample vial. The lysate was centrifuged for $10 \mathrm{~min}$ at $5000 \times \mathrm{g}$ at $4{ }^{\circ} \mathrm{C}$. The final decanted supernatant was used to make $10 \%(\mathrm{v} / \mathrm{v})$ crude cell lysate solution in $0.1 \mathrm{M}$ phosphate buffer solution ( $\mathrm{pH}$ 7.4).

4.2.5 Human cell extract preparation Human lymphoblast cell lines GM02152 (normal individual) and GM16207 (FRDA patient) were purchased from Coriell Institute for Medical Research (Camden, NJ) and cultured in RPMI 1640 medium supplemented with $15 \%$ fetal bovine serum and $2.05 \mathrm{mM} \mathrm{L}$-glutamine at $37^{\circ} \mathrm{C}$ under $5 \% \mathrm{CO}_{2}$. Cell pellets from normal or FRDA patient lymphoblasts were resuspended in lysis buffer that contained $10 \mathrm{mM}$ Tris- $\mathrm{HCl}(\mathrm{pH}$ 7.8) including $200 \mathrm{mM} \mathrm{KCl}, 1 \mathrm{mM}$ EDTA, 20\% glycerol, $0.1 \%$ Nonidet P-40, 1 mM DTT with proteinase inhibitors (Roche Diagnostics Corporation, Indianapolis, IN). Cell suspension was subsequently subjected to rotation at $4{ }^{\circ} \mathrm{C}$ for $2 \mathrm{hrs}$. After rotation, cell lysates were centrifuged at $14,000 \mathrm{rpm}$ at $4{ }^{\circ} \mathrm{C}$ for 30 min to remove cell debris. The supernatant was recovered and further dialyzed into buffer containing $50 \mathrm{mM}$ Tris- $\mathrm{HCl}(\mathrm{pH} 7.5)$ including $50 \mathrm{mM} \mathrm{KCl}, 0.1 \mathrm{mM}$ EDTA, $0.1 \mathrm{mg} / \mathrm{ml}$ bovine serum albumin, and $0.01 \%$ Nonidet P- 40 . Protein concentration of cell extracts 
was determined by Bradford assay. Cell extracts were stored at $-80{ }^{\circ} \mathrm{C}$ for subsequent assays.

\subsubsection{Saliva collection and preparation of the pooled saliva matrix Saliva samples} were collected from seven individuals from varying ethnic backgrounds both male and female, which are pooled together before processing. Resting drooling (minimal oral movements) was used to collect whole mouth saliva from the oral cavity with the head down slightly to pool saliva in the mouth. The subsequent sample was then expectorated into a pre-labeled sterile container and $\sim 5 \mathrm{~mL}$ saliva was collected. The collected saliva sample was centrifuged (Max. speed for 15 minutes) to remove cellular debris and to minimize the turbidity of saliva. The supernatant was transferred into a fresh Eppendorf tube for further analysis. Saliva thus obtained is used directly for the experiments or stored at $4{ }^{\circ} \mathrm{C}$ refrigerator for further use.

\subsubsection{EMIT assay and electrochemical detection of NADH production in saliva}

samples The enzymatic reaction was initiated by adding $90 \mathrm{uL}$ of the enzyme reagents (reagent-1 and reagent -2 ) in the optimized ratio to $4.5 \mathrm{uL}$ of undiluted biosample (saliva) or calibrators. The final ratio of reagent to sample was the same as described in the original assay package insert but with slight optimization for TECAN spectrophotometer. Sample dilution was 21-fold, as usual, for the enzyme assay with no further dilution. The mixture of the two reagents along with the sample after 30 minutes of incubation is directly measured for absorbance using TECAN and used as electrolyte in electrochemical system to measure the produced NADH. 


\subsection{Results and Discussions}

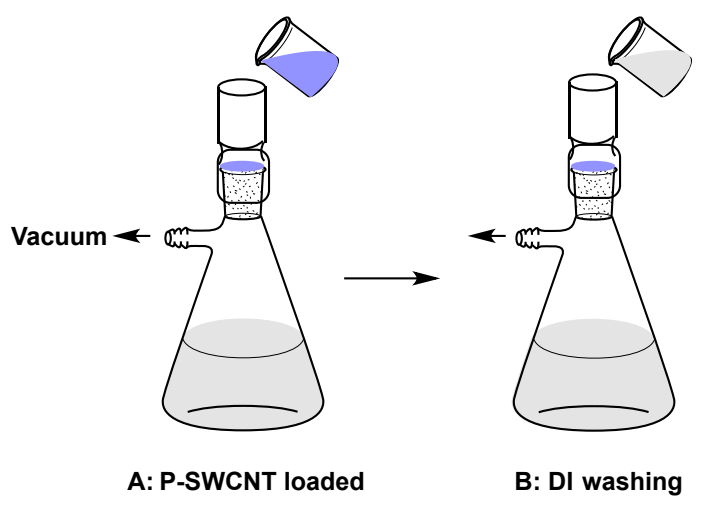

Figure 35: Preparation of Porous P-SWCNT film via vacuum filtration. (A) Vacuum filtration of a solution of $1 \%$ SDS-dispersed M-SWCNTs results in a compact and uniform P-SWCNT underlay on the paper surface. (B) SDS surfactant is washed away with copious amounts of deionized (DI) water to provide a clean hydrophobic surface.

A simple vacuum filtration technique enabled us to fabricate our thin P-SWCNT films on MCE paper substrate with minimal requirements of reagents, labor, and resources at room temperature that produced homogenous and reproducible films (Figure 35). A dispersed solution of our P-SWCNTs in 1\% SDS surfactant solution with variable carbon nanotube concentrations was used as the initial feed to deposit them onto a pre-wetted MCE filter paper (100 nm pore size) rested on a filtration system under vacuum force. A thin compact film of P-SWCNTs formed onto the surface of MCE paper by allowing the dispersed P-SWCNT solution pass through at a rate of $\sim 0.6 \mathrm{~mL} / \mathrm{min}$ under a low vacuum suction (Figure 35A). The P-SWCNT layer formed on the MCE paper was then washed with copious amounts of DI water to remove the residual surfactant from the carbon nanotube surface (Figure 35B), and let it dry at room temperature. Vacuum filtration forced the carbon nanotubes to form a compact layer on top of the MCE paper with maximum overlap thus resulting in a rigid and uniform film. 


\subsubsection{Effect of amount of P-SWCNT on conductivity and electron transfer rate of the P-SWCNT film}

Paper-based film with excellent conductivity and faster electron transfer capabilities with minimum material cost is essential in achieving a highly efficient electrode surface. To understand, optimize and evaluate the conductive properties of variable P-SWCNTs loaded MCE paper substrate, we conducted measurements of sheet resistance $(\Omega / \mathrm{sq})$ of various films (Figure 36) with a Keithly 4200 Semiconductor Characterization System with a four-point probe setup. As expected, the MCE paper itself was not conductive, and we observed infinite resistance during the measurement. Upon adding $12.5 \mu \mathrm{g}$ of PSWCNTs onto the MCE paper, a thin pale-grey film formed on its surface exhibiting a moderate conductivity $(\sim 4085 \Omega / \mathrm{sq})$. The conductivity of our P-SWCNT film along with its color intensity gradually increased to a darker shade with increasing amounts of PSWCNTs loaded onto the MCE paper. The relative increase $(60 \%)$ in the conductivity to $\sim 1602 \Omega /$ sq with $20 \mu \mathrm{g}$ P-SWCNT may be attributed to the increased density of carbon nanotubes forming an interconnected network on the surface of porous MCE paper. The conductivity of our film began to reach saturation $\sim 716 \Omega /$ sq at $50 \mu \mathrm{g}$ P-SWTCNT after which only a small increase of $4 \%$ was observed. 


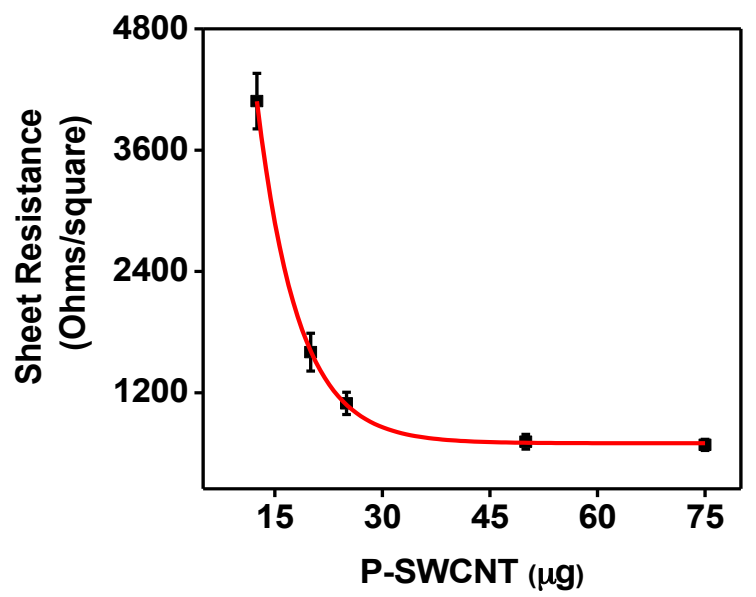

Figure 36: Effect of variable amounts of P-SWCNTs on sheet resistance of the fabricated film. Increasing amounts of P-SWCNTs (12.5-75 $\mu \mathrm{g})$ loaded on MCE paper resulted in a steady decline in sheet resistance, with values starting to bottom out at around $50 \mu \mathrm{g}$.

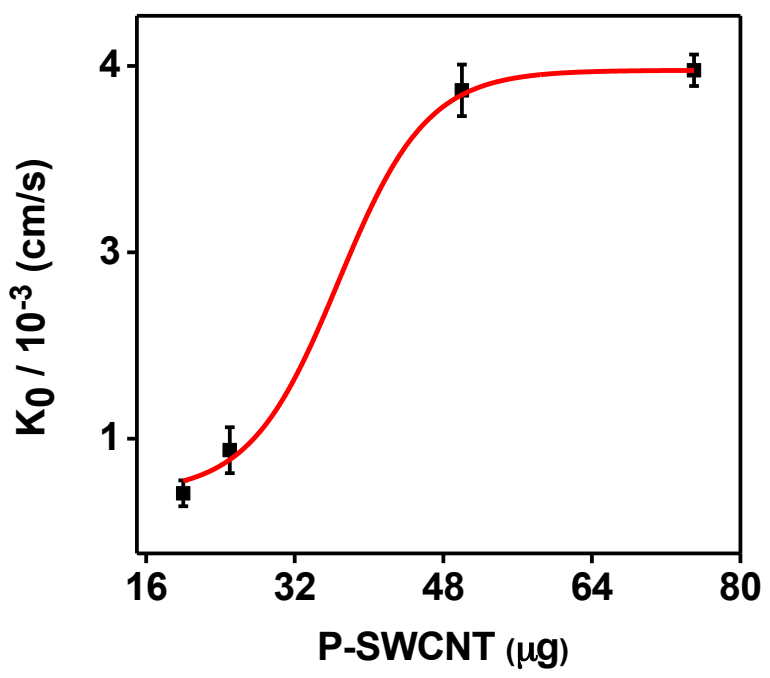

Figure 37: Effect of variable amounts of P-SWCNTs on electron transfer rate. Increasing amounts of P-SWCNTs on MCE paper were associated with a steady increase in electron transfer rate $\left(\mathrm{K}_{0}\right)$, with values starting to reach a plateau around $50 \mu \mathrm{g}$.

Thin P-SWCNT film electrodes were fabricated with dimensions of $2 \mathrm{~mm}$ width and 4 mm length and the electrode characteristics were studied by understanding the electron transfer kinetics. Electron transfer rate is described as the process of transfer of electrons between the electrode surface and its interfacial solution. It can be calculated by 
examining the peak-to-peak separation $(\Delta \mathrm{Ep})$ of $\mathrm{Fe}(\mathrm{CN})_{6}{ }^{3-} / \mathrm{Fe}(\mathrm{CN})_{6}{ }^{4-}$ in cyclic voltammograms using the Nicholson method $^{171}$, where a small $\Delta \mathrm{Ep}$ indicates faster electron transfer. As expected, MCE filter paper was non-conductive to electron transfer, and the behavior was extended to $12.5 \mu \mathrm{g}$ P-SWCNTs loading, exhibiting moderate conductivity. The $20 \mu \mathrm{g}$ P-SWCNT loading yielded an $\Delta \mathrm{Ep}$ of $113 \mathrm{mV}$ at a scan rate of $10 \mathrm{mV} / \mathrm{s}$, indicating a very low electron transfer rate constant (Figure 37), corroborating the change in conductivity presented in Figure 36 . However, the $\Delta$ Ep gradually decreased with increasing amounts of P-SWCNTs saturating around $75 \mathrm{mV}$ with a calculated electron transfer rate of $3.87 \times 10^{-3} \mathrm{~cm} / \mathrm{s}$ for $75 \mu \mathrm{g}$ P-SWCNT loading on the paper. The saturation kinetics of electron transfer however began to appear at $50 \mu \mathrm{g}$ P-SWCNT loading, yielding a $\Delta \mathrm{Ep}$ of $76 \mathrm{mV}$ at a scan rate of $10 \mathrm{mV} / \mathrm{s}$, with a calculated electron transfer rate of $3.73 \times 10^{-3} \mathrm{~cm} / \mathrm{s}$ showing a mere $2.6 \%$ change from its successor (Figure 37). The rate of electron transfer at the electrode surface is largely dependent on the structure and the morphology of the conductive material. The presence of higher amounts edge plane defects at higher P-SWCNT loaded films is responsible for increased electron transfer electron transfer rates however saturation of surface led to the plateau at $75 \mu \mathrm{g} \mathrm{P-}$ SWCNT.

The electrocatalytic activity of paper-based P-SWCNT film electrode along with its performance in complex biosample matrices serves as some of the important optimizing parameters in choosing the amount utilized to make our P-SWCNT film. The films prepared with different amounts of P-SWCNTs $(12.5,20,25,50$, and $75 \mu \mathrm{g})$ are tested for their electrocatalytic ability for detection NADH oxidation in buffer as well as some selected complex biological matrices. 


\subsubsection{Effect of amount of P-SWCNT on NADH electrocatalytic responses in PBS buffer}

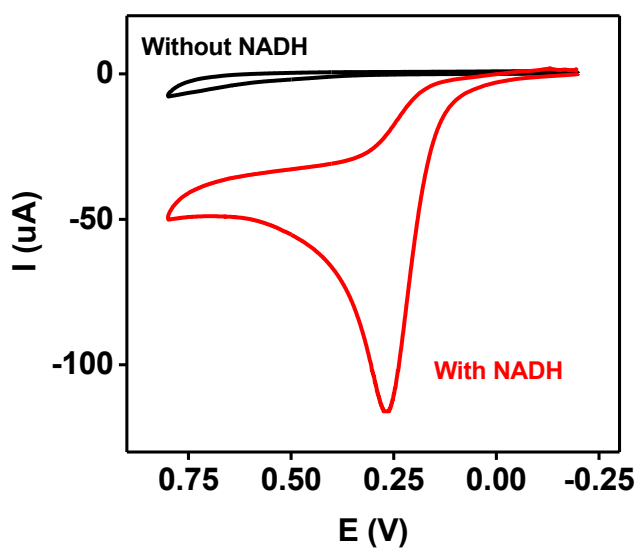

Figure 38: Effect of amount of P-SWCNT on NADH electrocatalytic responses in PBS buffer Cyclic voltammograms of P-SWCNT films in $0.1 \mathrm{M}$ PBS. (A) Electrochemical oxidation of NADH with $12.5 \mu \mathrm{g}$ P-SWCNT electrode in the presence and absence of 1 $\mathrm{mM}$ NADH. (B) Increase in the capacitance current observed with the increased PSWCNT loading on the surface of the MCE paper in 0.1 M PBS buffer.

All of our paper-based P-SWCNT films prepared with varying amounts of P-SWCNTs showed excellent electrocatalytic activity for NADH oxidation owing to their electrocatalytic surface areas and porous architectures formed as a result of vacuum filtration onto a porous MCE paper substrate. Among all P-SWCNT films tested, the highest electrocatalytic response for NADH oxidation in $0.1 \mathrm{M}$ PBS buffer against a very small capacitance current observed in the absence of NADH was seen for the lowest PSWCNT loading $(12.5 \mu \mathrm{g})$ (Figure 38). However, lowering the amount below $12.5 \mu \mathrm{g}$ resulted in the formation of non-uniform layer on the surface of the MCE paper.

The signal enhancement fold decreased gradually with increasing amounts of P-SWCNT (Figure 39A). This gradual decrease is attributable to the increase in the capacitance current in the absence of NADH at higher P-SWCNT loadings. However, there is a 
positive oxidation potential shift observed at lower P-SWCNT loadings indicating the requirement of higher energy for the oxidation reaction to occur (Figure 39B). The positive shift at lower P-SWCNT loadings may be attributed to the availability of limited catalytic sites fouled by the adsorption of NADH and $\mathrm{NAD}^{+}$on the surface. ${ }^{204} \mathrm{~A}$ signal enhancement of 77-fold was observed with $50 \mu \mathrm{g}$ P-SWCNT loaded film at a lower oxidation potential of $0.22 \mathrm{~V}$, which further decreased to 39 -fold with $75 \mu \mathrm{g}$ P-SWCNT loaded film at $0.17 \mathrm{~V}$. Based on above results, the paper-based PSWCNT film prepared with $50 \mu \mathrm{g}$ P-SWCNT was chosen as the optimum amount considering the factors such as signal enhancement, oxidation potential and material cost.
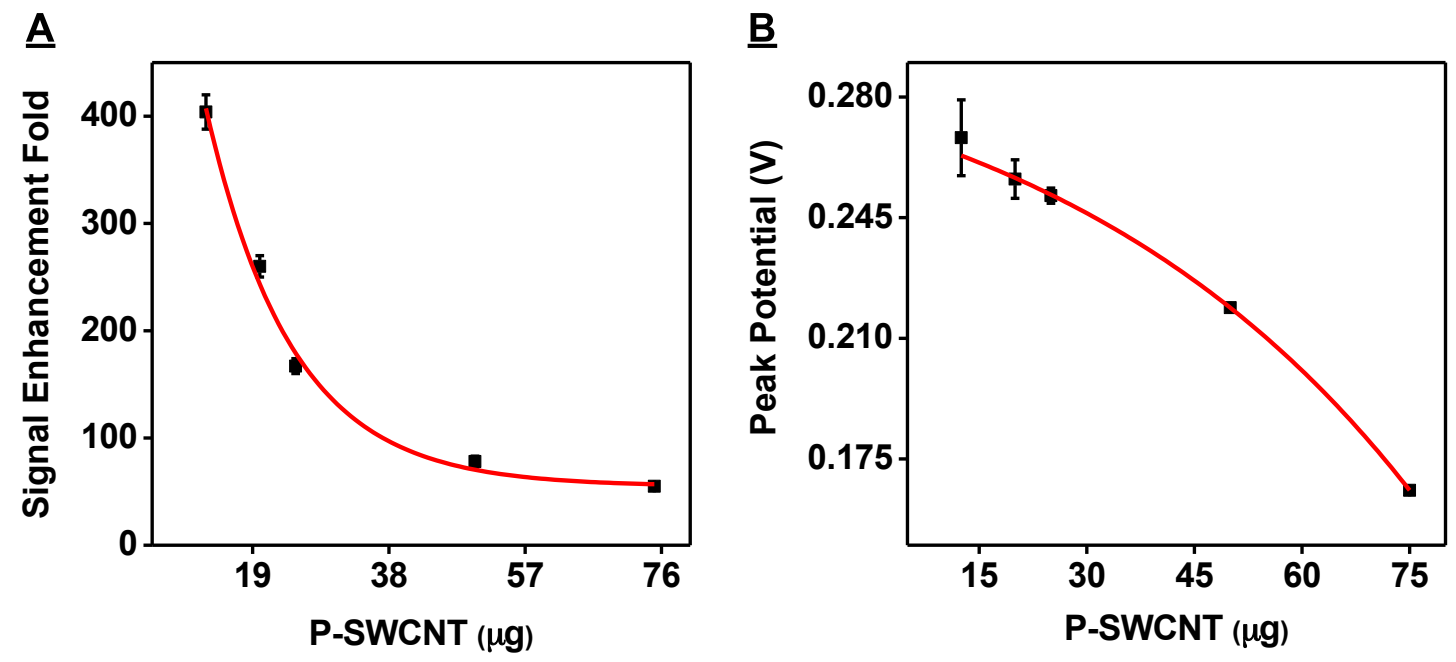

Figure 39: Comparison of the electrochemical catalytic responses of our various paperbased P-SWCNT films in 0.1 M PBS buffer using cyclic voltammetry. (A) Comparison of signal enhancement fold for electrochemical detection of $1 \mathrm{mM}$ NADH in $0.1 \mathrm{M}$ phosphate-buffered saline ( $\mathrm{pH}$ 7.4) (B) NADH oxidation peak potential shift observed with variable paper-based P-SWCNT films. 


\subsubsection{Effect of amount of P-SWCNT on NADH electrocatalytic responses in biosamples}

$\underline{A}$

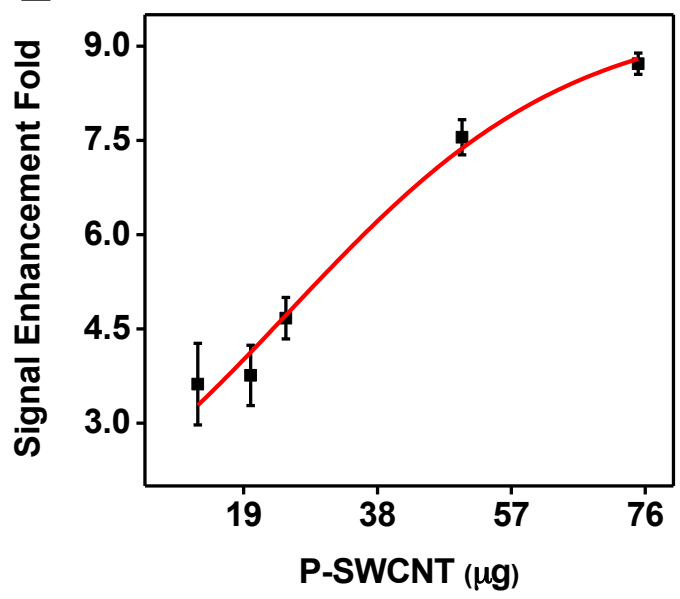

$\underline{B}$

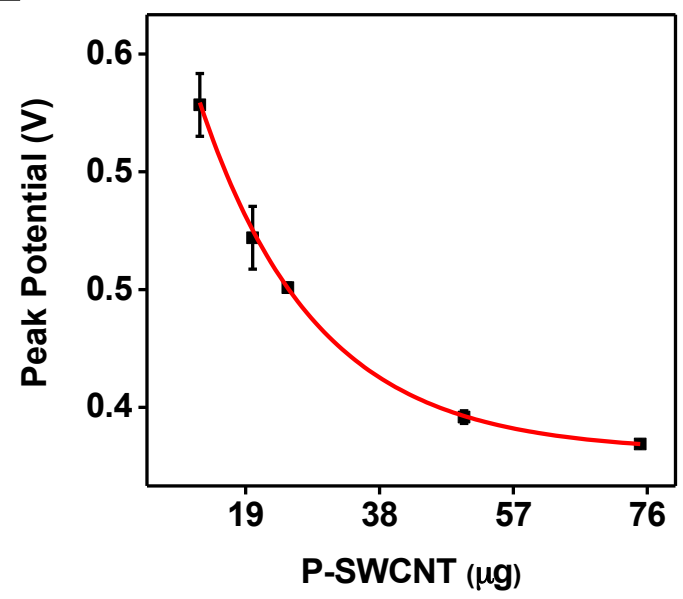

Figure 40: Comparison of the electrochemical responses of our various paper-based PSWCNT film electrodes in pooled saliva using cyclic voltammetry. (A) Comparison of signal enhancement fold for electrochemical detection of $1 \mathrm{mM} \mathrm{NADH}$ in $50 \%$ saliva (B) NADH oxidation peak potential shift observed with variable P-SWCNT films in 50\% saliva.

The $\mathrm{NAD}^{+} / \mathrm{NADH}$ redox couple is a significant cofactor participating in more than 300 dehydrogenase enzymatic reactions. ${ }^{83}$ The electrochemical oxidation of NADH at the electrode surface has garnered a lot of interest in the area of biosensors, because of the commercially available nature of these dehydrogenase enzymes, that can be applicable in clinical, environmental, and food analysis. ${ }^{80,83,97}$ Drug screening usually utilizes these dehydrogenase-based systems to generate NADH as a signal indicator for the presence of target. Biosample matrix such as saliva is probably the idea matrix for on-site drug detection owing to its non-invasive procurement, relatively clean, and ease of accessibility. Therefore, we evaluated the performance of our paper-based P-SWCNT film electrodes in saliva matrix. The choice of using $50 \mu \mathrm{g}$ P-SWCNTs as the optimumloading amount is further strengthened by the film's performance in saliva. Specifically, 
the films prepared with variable amounts of P-SWCNTs are tested for their electrocatalytic oxidation of $1 \mathrm{mM} \mathrm{NADH}$ in saliva. Saliva sample was pre-diluted to $50 \%$ with $0.1 \mathrm{M}$ PBS and spiked with $1 \mathrm{mM} \mathrm{NADH}$ to evaluate the signal enhancement performance (Figure 40A) and oxidation peak potential (Figure 40B) of different paperbased P-SWCNT film electrodes. We found that P-SWCNT film with $12.5 \mu \mathrm{g}$ gave the smallest signal enhancement of 3.5 fold at an oxidation potential of $0.57 \mathrm{~V}$. The signal enhancement however gradually increased with increasing amounts of P-SWCNT loadings at more negative oxidation potentials (Figure 40).

This shift in peak potential seen in 0.1 M PBS buffer because of the presence of inherent background peaks from the saliva sample matrix. The presence of proteins in the biosamples or the adsorbed $\mathrm{NAD}^{+}$can passivate the electrode surface at higher potentials, causing decreased current response. A signal enhancement fold of 7.5 was observed with $50 \mu \mathrm{g}$ P-SWCNT loaded film electrode at a lower oxidation potential of $0.37 \mathrm{~V}$, which further increased only by a fraction to 8.5 -fold with $75 \mu$ g loading at $0.35 \mathrm{~V}$. In addition, saliva sample matrix has inherent background as evident from the cyclic voltammogram with an interference oxidation peak at $0.2 \mathrm{~V}$, possibly arising due to ascorbic acid. ${ }^{207}$ When spiked with NADH, the interference peak does not interfere with NADH oxidation at $0.37 \mathrm{~V}$ (Figure 41). 


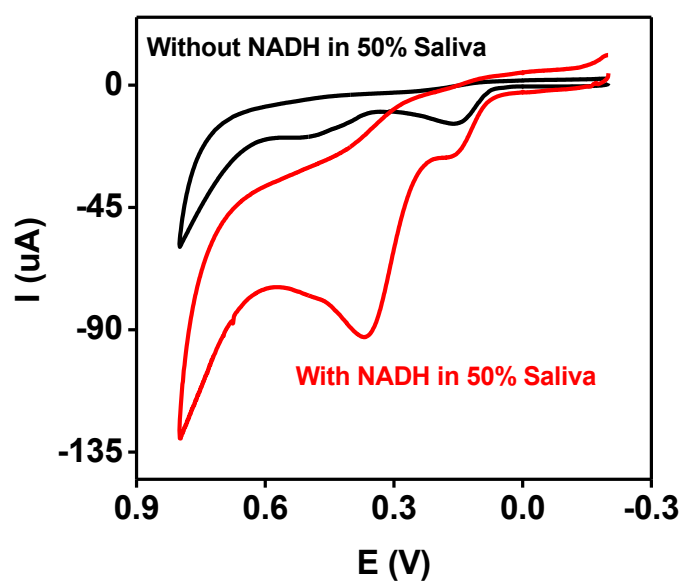

Figure 41: Cyclic voltammograms of $50 \mu \mathrm{g}$ P-SWCNT film in $50 \%$ saliva in the presence (red trace) and absence (black trace) of $1 \mathrm{mM} \mathrm{NADH}$.

\subsubsection{Characterization of surface morphology of paper-based P-SWCNT film}

The paper-based P-SWCNT film was further characterized by atomic force microscopy (AFM) to understand the morphology and transferred onto a clean glass substrate using acetone evaporation ${ }^{174}$ to measure the edge thickness. An edge thickness of $80 \mathrm{~nm}$ was obtained from the AFM analysis (Figure 42A) for our $50 \mu \mathrm{g}$ P-SWCNT loaded film indicating the formation of a thin compact layer on the surface of the MCE paper. The AFM image shows that our P-SWCNTs are randomly oriented on the surface of the MCE paper (Figure 42B). We used the average roughness $(\mathrm{Ra})^{172,173}$ parameter which is the mean height as calculated over the entire measured area, to detect general variations in overall height profile of our P-SWCNT film on paper (Figure 42B). A small measured Ra value of $10 \mathrm{~nm}$ indicates a smooth surface with uniformly embedded carbon nanotubes over the surface of the MCE paper as seen from the AFM morphology. 

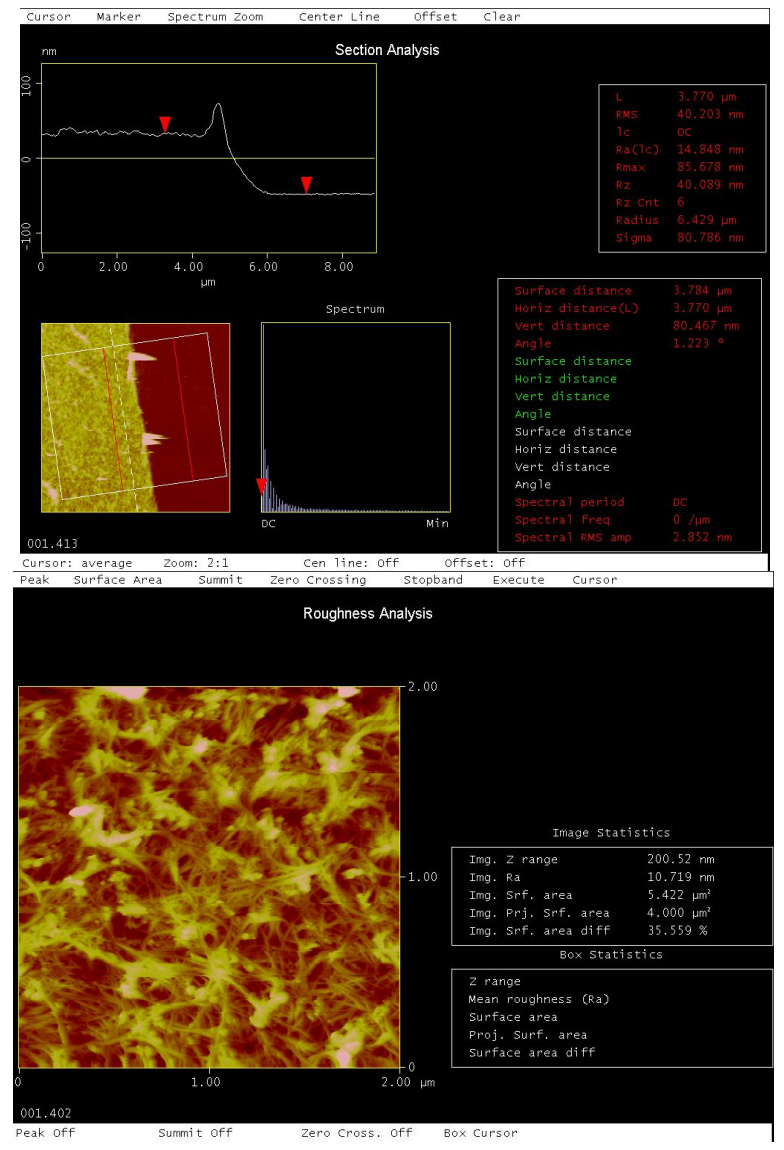

Figure 42: Surface morphology of our $50 \mu \mathrm{g}$ P-SWCNT loaded film as characterized by atomic force microscopy (AFM). (A) Typical AFM image analyzing the film edge to measure the thickness of the P-SWCNT layer. (B) AFM image analyzing the film morphology and surface roughness.

The films prepared with variable amounts of P-SWCNTs were tested for their electrocatalytic responses of NADH spiked in E Coli cell lysate diluted to $50 \%$ with 0.1 M PBS. The paper-based P-SWCNT film electrode loaded with $12.5 \mu \mathrm{g}$ gave the smallest signal enhancement fold (5.8) at an oxidation potential of $0.53 \mathrm{~V}$. The signal enhancement fold gradually increased with increasing amounts of P-SWCNT loadings at lower oxidation potentials (Figure 43A). This increase in signal enhancement fold is similar to the trend seen in $50 \%$ saliva buffer because of the presence of inherent background peaks from cell lysate. A signal enhancement fold of 9 was observed with 50 $\mu \mathrm{g}$ P-SWCNT loaded film electrode at a lower oxidation potential of $0.36 \mathrm{~V}$, which 
further decreased to 5.8 -fold with $75 \mu \mathrm{g}$ P-SWCNT loaded film electrode at $0.32 \mathrm{~V}$ (Figure 43B).
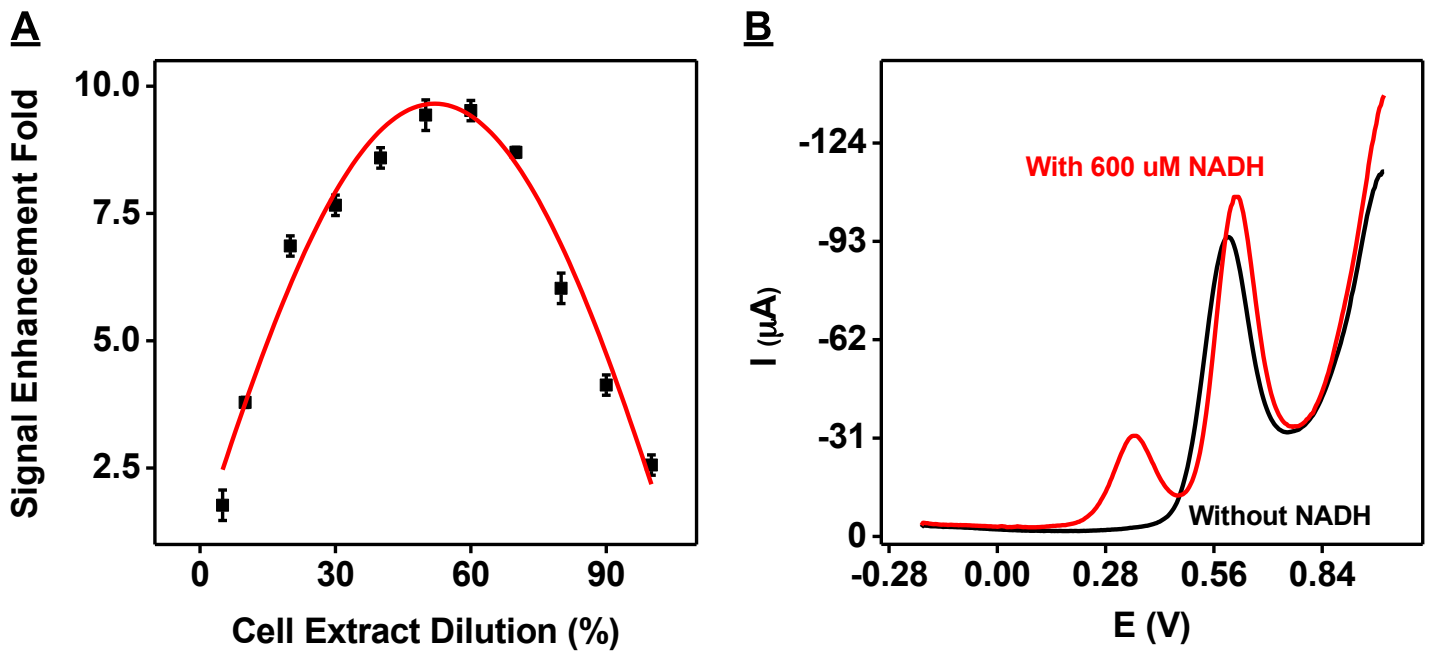

Figure 43: Effect of E. coli cell extract dilution for NADH oxidation on P-SWCNT electrode (A) Signal enhancement fold obtained from DPV for different dilution ratios of E. coli cell extract matrix with $0.1 \mathrm{M}$ PBS and $1 \mathrm{mM}$ NADH. (B) DPV of $50 \mu \mathrm{g}$ PSWCNT film at $60 \%$ cell extract in the presence and absence of $600 \mu \mathrm{M}$ NADH.

The lysate obtained from Human lymphoblast cell lines GM02152 (normal individual) and GM16207 (FRDA patient) is chosen as another complex matrix to evaluate the electrocatalytic signal of our paper-based P-SWCNT film electrodes for disease diagnosis as intracellular NADH levels can serve as a biomarker for abnormal conditions. ${ }^{71-77}$ As it is evident from the figure below (Figure 44) showing a noticeable spike in the peak current at $0.62 \mathrm{~V}$ for FRDA cell extract using $50 \mu \mathrm{g}$ P-SWCNT loaded film electrode possibly arising because of higher levels of NADH compared to the normal cells. 


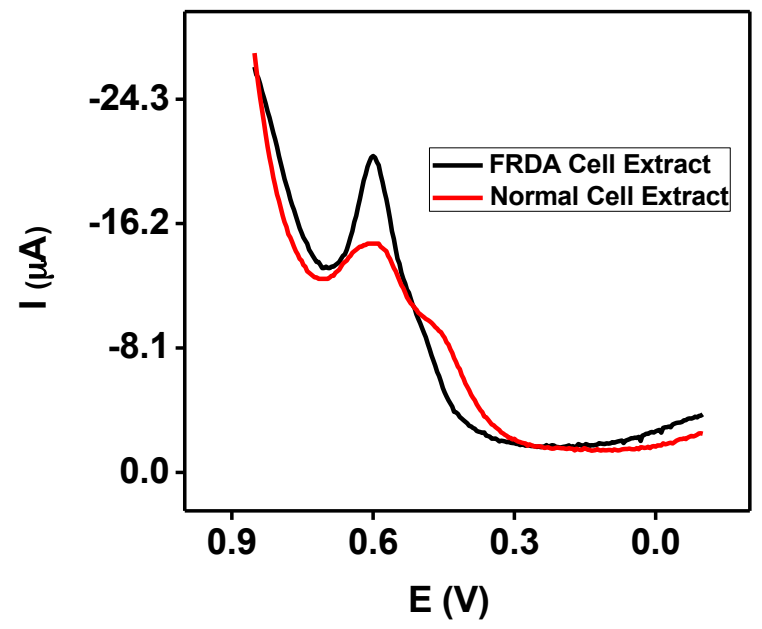

Figure 44: Differential pulse voltammograms of $50 \mu \mathrm{g}$ P-SWCNT film in un-diluted human cell extract after dialysis from normal (red trace) and FRDA patient (black trace) lymphoblasts.

\subsubsection{Paper-based P-SWCNT film electrodes for detection of enzyme-generated}

\section{NADH in saliva}

In order to evaluate the performance of our paper-based P-SWCNT film electrode in electrochemical oxidation of enzyme-produced NADH, we first spiked NADH (1 mM) into $100 \%$ saliva followed by serial dilutions in $0.1 \mathrm{M}$ PBS buffer to investigate different dilution effects. Differential pulse voltammetry (DPV) technique was used to detect oxidation of $\mathrm{NADH}$, instead of cyclic voltammetry $(\mathrm{CV})$ to increase the sensitivity and signal gain while working in complex biosamples. We found that using $50 \mu \mathrm{g}$ P-SWCNT loaded film as a working electrode, $40 \%$ dilution of saliva matrix [e.g. $400 \mu \mathrm{M}$ NADH] gave the optimum signal enhancement fold with the spiked NADH (Fig.10A). NADH peak at $0.38 \mathrm{~V}$ with $400 \mu \mathrm{M}$ NADH concentration (Figure 45, red trace) can be clearly distinguished from the saliva interference peaks in the background (Figure 45, black trace) with $40 \%$ dilution. 
$\underline{A}$

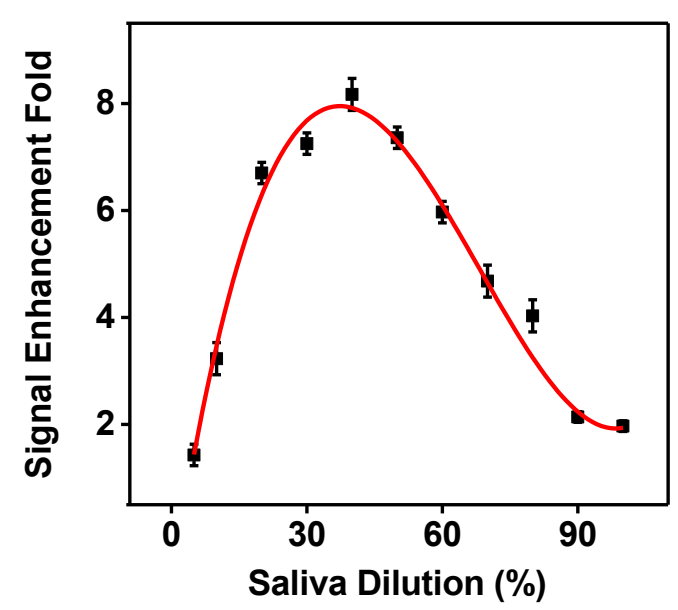

$\underline{B}$

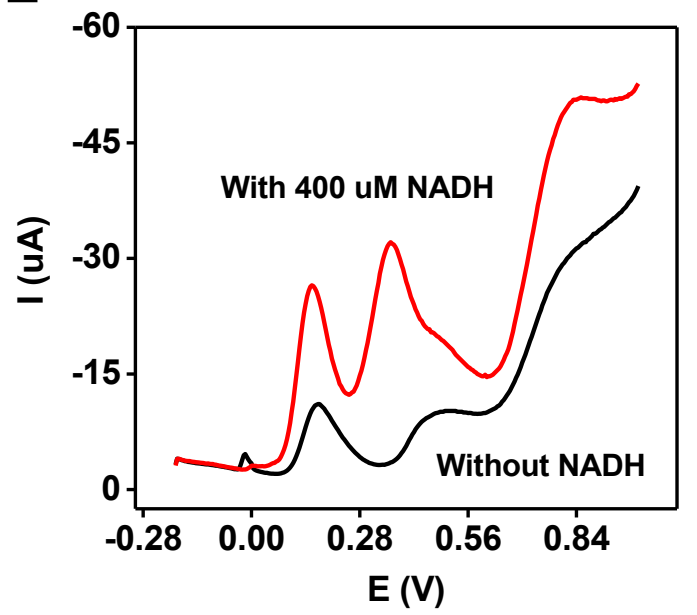

Figure 45: Effect of Pooled saliva dilution for NADH oxidation on P-SWCNT electrode obtained from DPV (A) Signal enhancement fold of different dilution ratios of saliva matrix with $0.1 \mathrm{M}$ PBS and $1 \mathrm{mM}$ NADH. (B) DPV of $50 \mu \mathrm{g}$ P-SWCNT loaded film in $40 \%$ saliva dilution with or without $400 \mu \mathrm{M}$ NADH.

We then tested enzyme-produced NADH with both spectrophotometry and electrochemistry. A commercial immunoassay kit $^{112}$ has been used to evaluate the performance of our $50 \mu \mathrm{g}$ P-SWCNT loaded film to detect NADH produced as a result of enzymatic reaction from the Syva reagents and cocaine metabolite (benzoylecgonine) in the biosample. 

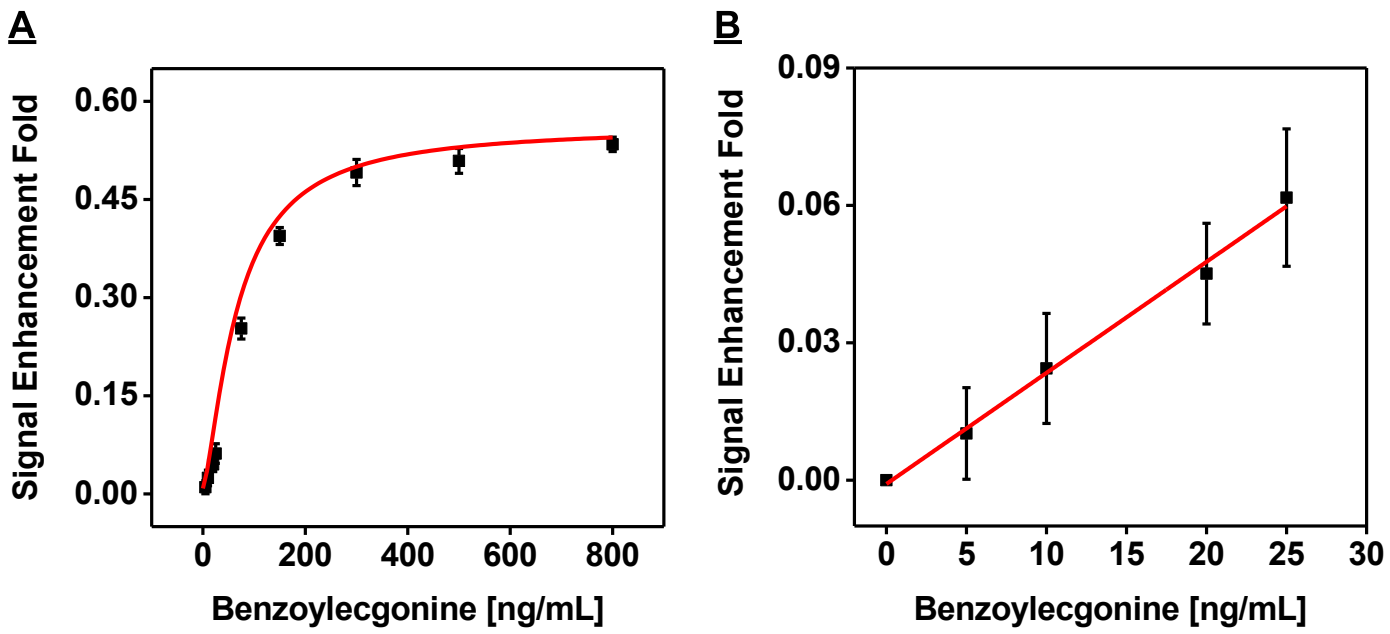

Figure 46: A hyperbolic response was observed for the enzyme-produced NADH as the concentration of benzoylecgonine increased from $5 \mathrm{ng} / \mathrm{mL}$ to $1000 \mathrm{ng} / \mathrm{mL}$. The measurements were performed with TECAN spectrophotometer at $340 \mathrm{~nm}$.

In the absence of drug, some enzyme-labeled drug molecules do remain free to generate $\mathrm{NADH}$, resulting in a background response at $340 \mathrm{~nm}$. Different concentrations of benzoylecgonine were spiked into saliva to test the linearity of response for enzymegenerated NADH as a function of drug concentration in the saliva. The saliva samples with or without benzoylecgonine were added to the EMIT reagents to generate NADH after competitive binding with the antibody. The analysis was done both by using a TECAN spectrophotomer (Figure 46) and electrochemistry (Figure 47) that was performed with our $50 \mu \mathrm{g}$ P-SWCNT loaded film electrode. A hyperbolic response was observed as the concentration of benzoylecgonine increased from $5 \mathrm{ng} / \mathrm{mL}$ to 1000 ng/mL using both TECAN spectrophotomer and our paper-based P-SWCNT film electrodes. 
$\underline{A}$

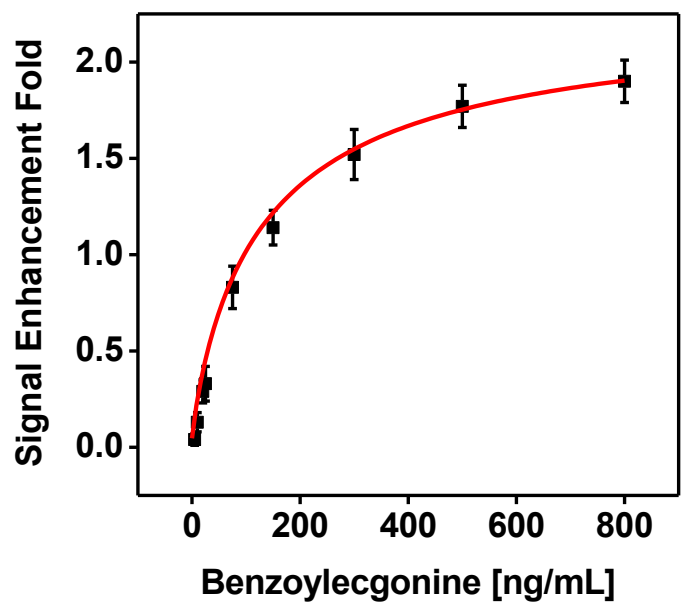

$\underline{B}$

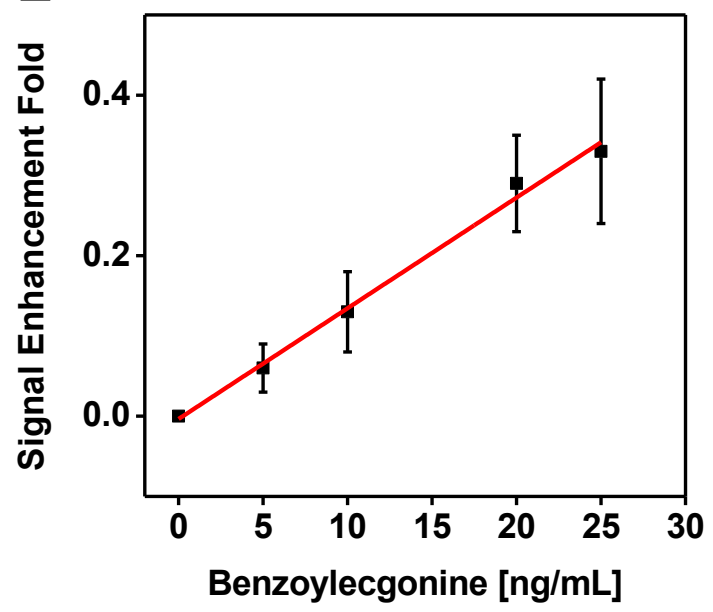

Figure 47: A similar hyperbolic response was observed for the enzyme-produced NADH as the concentration of benzoylecgonine increased from $5 \mathrm{ng} / \mathrm{mL}$ to $1000 \mathrm{ng} / \mathrm{mL}$. The measurements were performed with electrochemistry using 50 ug P-SWCNT loaded film electrodes.

\subsection{Conclusions}

In this chapter, we challenged our porous P-SWCNT film electrodes with the electrochemical detection of both naturally available and enzyme-generated reduced form of beta-nicotinamide adenine dinucleotide (NADH) in complex matrices such as saliva, and cell extracts. Quantification of $\mathrm{NADH}$ in these complex matrices provided us valuable information for disease diagnosis, drug detection and often comparable with the commercial colorimetric assays. Using filter paper as a substrate to support the nanomaterials add to the virtues of the composite film, making it flexible, cheap, and biodegradable. Meanwhile, utilizing the macroporous paper matrix to trap the nanotubes during the vacuum filtration provides a suitable and simple film-fabrication strategy. These facts offer a boarder application in the disposable NADH biosensors without modification of P-SWCNT electrode surface. 


\section{CHAPTER 5: Evolution of Probe Sequence Design Using Split Aptamers to Facilitate Efficient Enzyme Amplified Target Recycling for Onsite Colorimetric Detection of Cocaine}

\subsection{Introduction}

Rapid, simple and sensitive detection of drugs onsite with limited and portable instrumentation remains a challenge in today's fast paced world. Colorimetric methods and sensors are some of the oldest measuring principles used in the laboratories, where the color change can be readily visualized and interpreted for the presence or absence of a particular analyte in the sample. ${ }^{208}$ Gold nanoparticles (AuNPs) have a unique surface plasmon resonance absorption tends to change color depending on their binding or nonbinding with certain moieties in their local environment resultant of a change in their physical properties. ${ }^{209}$ DNA aptamers have emerged as a promising alternative to antibodies, as they are specific binding to proteins or small-molecule targets with high affinity, ${ }^{210,211}$ and have the benefit of being chemically synthesized rather than produced in vivo. ${ }^{123}$ The conjugation between AuNPs and DNA aptamers ${ }^{212}$ has wide applications in detection strategies owing to their novel catalytic, electronic, and optical properties. ${ }^{213}$, ${ }^{214}$ Usually, the attachment was well-established thiol adsorption chemistry ${ }^{215[1,2]}$ where multiple DNA strands can be attached onto the surface of the AuNPs.

The number of DNA strands on the surface of the AuNP plays a significant role in the sensitivity, reaction time and window of a biosensor system as the surface probe density dictates the hybridization efficiency with the target owing to the accessibility constraints caused by steric hindrance, and electrostatic repulsions between them. This hybridization efficiency will ultimately affect the reaction time and sensitivity. At high surface- 
coverage situations the electrostatic repulsions may hinder the accessibility of the target to bind with the probe resulting in poor hybridization efficiency and longer reaction time. ${ }^{216,217}$ On the other hand, very low DNA surface densities also are not favorable for the reaction as the conformation of the probes may attain a flattened architecture due to the strong interaction with the target, which can delay the degradation by nucleases. ${ }^{218}$ Therefore it is essential to optimize the probe density to achieve efficient hybridization, sensitive detection, and fast reaction times. This surface density of DNA on AuNP can be optimized using a well-established two-step ligand-exchange reaction using sequential addition of thiolated DNA probes followed by varying concentrations of a smaller thiolated compound such as DTT or MCH. ${ }^{219-222}$ The addition of DTT possessing two thiol functional moieties and hydrophilic nature can bind strongly to the gold surface, readily displacing the immobilized probe DNA from the surface of the AuNP. The displacement efficiency of DTT is directly proportional to its concentration in the ligandexchange reaction.

The objective of this chapter is to evaluate and understand the effect of different aptamer sequences on the efficiency of enzyme-assisted target recycling (EATR) to enable colorimetric onsite detection of cocaine. Cocaine is a schedule-II drug that is highly addictive stimulant, having the potential to alter the structure and function of the brain when used repeatedly. ${ }^{108}$ National Survey on Drug Use and Health (NSDUH) published the statistics showing a steady abuse of cocaine since 2009, rampant in the age-group of 18-25 year adults, translating to nearly one in three emergency room visits that involved cocaine in 2011. ${ }^{109}$ Cocaine abuse can lead to several health complications such as psychosis, anxiety, paranoia, heart attacks, seizures, abdominal pain, nausea, or even 
death in some instances. ${ }^{110}$ There have been reports of intracerebral hemorrhage causing bulges in the walls of cerebral blood vessels that can occur due to long-term cocaine use. ${ }^{111}$ Therefore it is important to detect and apprehend cocaine users from bringing harm to them and people around them. A lot of research has been going on to develop different techniques involving both screening as well as confirmatory tests to detect cocaine and its metabolites qualitatively and quantitatively in biofluids. ${ }^{112}$ Immunoassays among them are one of the widely used presumptive screening techniques such as enzyme-linked immunosorbent assay (ELISA), ${ }^{119}, 120$ enzyme-multiplied immunoassay technique (EMIT), ${ }^{112}$ radioimmunoassay (RIA), ${ }^{113}$ and fluorescence polarization immunoassay (FPIA), ${ }^{121}$ using antibodies, to detection of cocaine and its major metabolites. Although immunoassays such as ELISA provide the ease for quick screening, cross-reactivity with structurally similar compounds can generate false positives apart from being expensive, laborious, and time-consuming. ${ }^{120}$ Immunoassays such as EMIT although offer a cheaper alternative, were still hampered by the lack of sensitivity. ${ }^{223}$ Immunoassay techniques involving RIA enables sensitive detection, however required to handle the expensive radioactive waste materials therefore needed special training. ${ }^{113}$ Confirmatory tests based on mass spectrometry (MS) on the other hand are highly accurate, quantitative, and are considered gold standard in detection of cocaine in biofluids. ${ }^{112-118}$ The high specificity and reliability of the MS instruments has a downside for being expensive requiring a designated lab set-up with the expertise of trained personnel to analyze and interpret the results. Though the sensitivity, and specificity of the MS techniques are unparalleled, working with thousands of samples with these techniques is laborious, time consuming and very expensive. ${ }^{14-118}$ Therefore it 
is of paramount importance to develop an alternative screening method that can incorporate the advantages of immunoassays, while being fast, specific, relatively inexpensive, and less laborious thereby serving the purpose of rapid screening.

The use of aptamers and aptamer-based sensors revolutionized the field of small molecule detection and nucleic acid therapeutics. ${ }^{122}$ Aptamers are chemically synthesized, short, single-stranded (ss) RNA or DNA oligonucleotides that can bind specific targets via hydrogen bonding, electrostatic or ven der waals forces, possessing dissociation constants usually in the pico- to nano-molar range. ${ }^{211}$ Isolation of aptamers with high binding proclivity for small molecules, proteins, or even whole cells was made conceivable via in vitro selection of oligonucleotides (termed SELEX: systematic evolution of ligands by exponential enrichment). ${ }^{123}$ Aptamers can rival antibodies due to the huge discrimination showed by aptamers even in case of very closely related structures highlighting their huge potential in therapeutic, bioanalytic, and diagnostic applications. ${ }^{124-133}$ Compared to monoclonal antibodies aptamers were proven to have good binding affinity to their targets and assure high reproducibility, purity, stability even after repeated cycles of denaturation and regeneration, which is not true in case of proteins at elevated temperatures. ${ }^{134}$ The expense of screening large number of colonies in production of monoclonal antibodies is laborious and requires massive mammalian cell culture and the activity of antibodies varies from batch to batch, ${ }^{224}$ whereas aptamers once selected, can be reproduced with great accuracy via chemical reactions. Aptamers also offer great flexibility in design, and chemical modification as they undergo pronounced conformational change upon binding to the target, which elevates sensitivity and selectivity. ${ }^{225}$ 
Many researchers over the past decade have started developing aptamers for drugs of abuse. Recent work from Ebrahimi is the first in-vitro selection successfully reported for methamphetamine aptamer with $\mathrm{K}_{\mathrm{d}}$ in $\mathrm{nM}$ range. ${ }^{136}$ The first cocaine binding aptamer (MNS-4.1) was isolated Stojanovic had very good specificity to the parent cocaine as opposed to its metabolites and used in a fluorescence-based detection platform. ${ }^{131}$ This MNS-4.1 aptamer in theory undergoes a conformational change in the presence of cocaine, however the equilibrium between the folded and the unfolded states found in practice led towards higher background signals, limiting the sensitivity of the aptamer. ${ }^{131}$, ${ }^{226}$ To address this issue, MNS-4.1 was subsequently split into multiple aptamer fragments $\left(2^{130}\right.$ or $\left.3^{143,}{ }^{144}\right)$ that remain separated in the absence of cocaine, however come together in the presence of cocaine, thereby reducing the background. Although the background is reduced with the split aptamer the sensitivity remained limited owing to the low binding affinity with single binding domain. ${ }^{227}$ To challenge this problem a cooperative binding-based approach is developed with multiple binding domains, where binding at one domain enhances the affinity of the other domain, resulting in a 'switchlike' binding curve. ${ }^{156}$

In this chapter, aptamer sequences were evaluated mainly on three basic criteria, the hybridization efficiency, specificity towards the analyte (cocaine), and the reaction time to observe a distinguishable color change from red to blue. The split cocaine-binding aptamer was chosen as a model to understand the above-mentioned phenomenon involving probe-aptamer design for Exo III-assisted amplification systems and its ability to digest the aptamer-target complex on the surface of AuNPs. 


\subsection{Materials and Methods}

Gold(III) chloride trihydrate, trisodium citrate dehydrate, Tris(2-carboxyethyl)phosphine hydrochloride, sodium chloride, magnesium chloride, Trizma pre-set crystals, hydrochloric acid, sodium hydroxide, 1,4-Dithiothreitol, cocaine hydrochloride, TrisEDTA buffer were purchased from Sigma-Aldrich and used as received. Quant-iTTM OliGreen ${ }^{\circledR}$ ssDNA Reagent was purchased from Thermo Fisher Scientific and aliquot upon arrival. Exonuclease III (E. coli) was purchased from New England BioLabs and aliquot upon arrival. Amicon Ultra-4 Centrifugal Filter columns (100KD) were purchased from Millipore. All the solutions were prepared with distilled water $\left(18.2 \mathrm{M}^{\mathrm{cm}} \mathrm{cm}^{-1}\right)$ from a Milli-Q Direct-8 water system unless otherwise specified. All DNA sequences were synthesized by Integrated DNA Technologies (USA), purified with HPLC and confirmed by mass spectrometry. The list of DNA sequences used is in Table 1.

Table 2. DNA sequences employed in this work.

\subsubsection{Thiolated short aptamer fragmets:}

(S-1) SF-SSA: 5'-HS-C6-TTT TTT GAG ACA AGG/iSpC3/ ACA AGG AG -3'

(S-2) SF-5345: 5'-HS-C6-TTT TTT GAG ACA AGG/iSpC3/ AGA CAA GGA G -3'

(S-3) SF-5335: 5'-HS-C6-TTT TTT GAG ACA AGG /iSpC3/ GAC AAG GAG -3'

(S-4) SF-5335-10T: 5'-HS-C6-TTT TTT GAG ACA AGG /iSpC3/ GAC AAG GAG TTT TTT TTT T -3'

(S-5) S-5335-2X: 5'-HS-C6-TTT TTT GAG ACA AGG /iSpC3/ GAC AAG GAG TTT TTT GAG ACA AGG/iSpC3/ GAC AAG GAG -3'

\subsubsection{Long aptamer fragments:}

LF-225: 5'-CTC CTT CAA CGA AGT GGG TTC C -3' 
LF-425: 5'-CTC CTT CAA CGA AGT GGG TTC CTT -3'

LF-525: 5'-CTC CTT CAA CGA AGT GGG TTC CTT G -3'

LF-725: 5'-CTC CTT CAA CGA AGT GGG TTC CTT GTC -3'

LF-925: 5'-CTC CTT CAA CGA AGT GGG TTC CTT GTC TC -3'

LF-5325: 5'-CTC CTT CAA CGA AGT GGG TTC CTT CAA CGA AGT GGG TCT C $3^{\prime}$

LF-5345: 5'-CTC CTT CAA CGA AGT GGG TCT TCC TTC AAC GAA GTG GGT CTC $-3^{\prime}$

LF-5335: 5'-CTC CTT CAA CGA AGT GGG TCT CCT TCA ACG AAG TGG GTC $\mathrm{TC}-3^{\prime}$

5.2.3 Synthesis of AuNPs: Preceding the synthesis of AuNPs solution, all glassware was incubated in $\mathrm{HNO}_{3}-\mathrm{HCl}(3: 1 \mathrm{v} / \mathrm{v})$ overnight at room temperature, and washed with deionized (DI) water. Fren's citrate reduction of $\mathrm{HAuCl}_{4}$ was utilized to set up the AuNPs, with sodium citrate going about as both reducing and stabilizing operator. ${ }^{166}$ Boiling DI water $(45 \mathrm{~mL})$ was mixed with freshly dissolved and filtered $0.1 \mathrm{M}$ $\mathrm{HAuCl}_{4} 3 \mathrm{H}_{2} \mathrm{O}(5 \mathrm{~mL}$ in DI water) in a round bottomed flask under energetic mixing in a reflux setup. At that point, $38.8 \mathrm{mM}$ aqueous sodium citrate $(5 \mathrm{~mL})$ was quickly added into the $\mathrm{HAuCl}_{4}$ solution with nonstop mixing to reduce the oxidized $\mathrm{Au}^{3+}$ species to form AuNPs with a diameter of $\sim 12 \pm 1 \mathrm{~nm}$. Once the solution changed shading to red, it was continued boiling for another 10 min subsequently cooled down to room temperature and filtered utilizing a $0.22 \mu \mathrm{m}$ syringe filter for immediate use. The concentration of AuNP solution was measured using a Cary 100 Bio UV-Vis spectrophotometer with a characteristic absorbance peak at $\sim 520 \mathrm{~nm}$. 
5.2.4 Modification of thiolated short aptamer fragment on AuNPs: The modification of thiolated short aptamer fragment on the surface of AuNP was performed using a wellknown method utilizing gold-thiol chemistry. ${ }^{229}$ Prior to the modification all glassware and stirbars were incubated in $\mathrm{HNO}_{3}-\mathrm{HCl}$ solution $(3: 1 \mathrm{v} / \mathrm{v})$ overnight, and followed by incubation with $12 \mathrm{M} \mathrm{NaOH}$ solution to minimize the adsoption of AuNPs onto the walls of the glassware and stirbar during salt aging. The thiolated short aptamer fragment with a measured concentration (7.95 nmoles) was mixed with freshly prepared solution of Tris(2-carboxyethyl)phosphine hydrochloride (TCEP) (601.8 nmoles) in deionized water ( $3 \mathrm{mg} / 100 \mu \mathrm{L})$ at room temperature and stored in a dark place for at least 2 hours. The disulphide functionality of the oligonucleotide probes was cleaved by the addition and incubation with TCEP into a reduced form, ${ }^{220,230}$ that is then added to the AuNP $(3 \mathrm{~mL}, 9$ $\mathrm{nM})$ solution in small amounts $(10 \mu \mathrm{L})$ under continuous stirring at maximum speed. The final AuNP solution obtained after the complete addition of aptamer fragment was covered loosely with a cap and protected from light with an aluminum cover. Salt aging was performed by the addition of $4 \mathrm{M}$ sodium chloride to the AuNP solution in 3 installments after 12, 6, and 6 hours time difference between each addition adding 50, 100 , and $100 \mu \mathrm{L}$ respectively to reach a final $\mathrm{NaCl}$ concentration of $0.3 \mathrm{M}$. The stirring process is continued at room temperature in the dark for another 12 hours after the last salt addition, after which the resultant aptamer-modified AuNPs were washed, filtered, and concentrated using a centrifugal filter $(100 \mathrm{KD}) 1000 \mathrm{rcf}, 4{ }^{\circ} \mathrm{C}$ for 10 minutes for a total of 6 times. The supernatant solution after each washing step was collected to measure the DNA concentration, whereas the retained pellet in the centrifugal filter was 
resuspended with $1 \mathrm{~mL}$ of Trizma buffer (10mM, $\mathrm{pH} 7.4)$. The aptamer-modified AuNPs were finally re-suspended in Trizma buffer to achieve a final particle concentration of $100 \mathrm{nM}$ and stored at $4{ }^{\circ} \mathrm{C}$ refrigerator until further use.

\subsubsection{Characterization of short aptamer fragment density on the surface of AuNP} before and after DTT-displacement: To perform the assay to determine the surface coverage of short aptamer fragment, a set of calibration standard solutions of thiolated short aptamer fragment were prepared in $1 \times$ Trizma buffer $(\mathrm{pH} 7.4)$, ranging from 0 to 1 $\mu \mathrm{M}$ with a volume of $25 \mu \mathrm{L}$ in each tube. DTT displacement was performed by adding different concentrations of DTT to the aptamer-modified AuNPs, with a control prepared in $10 \mathrm{mM}$ Trizma buffer ( $\mathrm{pH}$ 7.4). The addition of DTT possessing two thiol functional moieties and hydrophilic nature can bind strongly to the gold surface, readily displacing the immobilized probe DNA from the surface of the AuNP. The displacement efficiency of DTT is directly proportional to its concentration in the ligand-exchange reaction. The samples were then placed on the rotator at room temperature for $30 \mathrm{~min}$ to let the DTT displacement reaction to completion followed by removing the displaced DNA and excess DTT in the supernatant and washing with $10 \mathrm{mM}$ Trizma buffer (pH 7.4) twice. To characterize the surface coverage of the DTT-treated, aptamer-modified AuNPs, the pellet was resuspended to $25 \mu \mathrm{L}$ of $10 \mathrm{mM}$ Trizma buffer ( $\mathrm{pH} 7.4$ ), to which $75 \mu \mathrm{L}$ of $1 \mathrm{M}$ DTT solutions was added both to samples and standard solutions. All the samples and standards were placed on the rotator for 12 hours after which $20 \mu \mathrm{L}$ of the supernatant was collected from the sample tubes after centrifugation at 25,000 rcf for $10 \mathrm{~min}$. QuantiT ${ }^{\mathrm{TM}}$ OliGreen ${ }^{\circledR}$ ssDNA Reagent has strong specific binding to ssDNA with a strong fluorescence emission at $525 \mathrm{~nm}$. To all the standards along with the samples in a 384- 
well microtitre plate, $80 \mu \mathrm{L}$ of Quant-iT ${ }^{\mathrm{TM}}$ OliGreen ${ }^{\circledR}$ ssDNA Reagent $1 \times$ was added and fluorescence intensities were measured at $525 \mathrm{~nm}$ using a TECAN M1000 pro spectrophotometer. The fluorescence intensities of the samples were extrapolated to the concentrations from the standard curve, and the respective probe surface coverage was calculated by dividing with the concentration of AuNPs.

\subsubsection{Effect of the surface density of short aptamer fragment modified AuNPs on}

Exo III-assisted cocaine recycling: To understand the effect of probe densities on the efficiency of an aptamer-target complex and exonuclease digestion in the presence of analyte, we first treated the short aptamer fragment modified-AuNPs with different concentrations of DTT as described above. The cocaine target and the long aptamer fragment $(100 \mathrm{nM})$, were then introduced into $10 \mathrm{mM}$ Trizma buffer ( $\mathrm{pH}$ 7.4) solution along with $\mathrm{NaCl}(100 \mathrm{mM})$ and $\mathrm{MgCl}_{2}(1 \mathrm{mM})$ comprising of the reaction buffer. After the DTT displacement, half of the sample mixture with the probe and target was spiked with cocaine solution $(250 \mu \mathrm{M})$ whereas the other half with deionized water for control in a microtitre plate. The samples and control were incubated at room temperature. After 30 minutes, Exo III $(20 \mathrm{U} / 100 \mu \mathrm{L})$ was added to all the solutions where the digestion of the aptamer-target complex in the presence and absence of cocaine was measured spectrophotometrically over a time period by recording the absorbance ratio at 650 to 522 $\mathrm{nm}\left(\mathrm{A}_{650} / \mathrm{A}_{522}\right)$ as an indicator of the level of AuNP aggregation. Different sequence modifications on the short aptamer fragment, the long aptamer fragment, and target concentration and their effect on the efficiency of cocaine-induced, Exo III-assisted target recycling were studied in a similar manner as described above with the exception of a fixed or optimized DTT concentration and variable long fragment concentration. 


\subsection{Results and Discussions}

Gold nanoparticles are one of the most widely used colorimetric reporters owing to their unique localized surface plasmon resonance (LSPR) absorption properties and their ability to change color depending on their local environment. ${ }^{231-233}$ It is therefore necessary to characterize the size and shape of the synthesized AuNPs before their use in further modifications. A modified Turkevich method ${ }^{23}$ has been used to synthesize AuNPs, after which they were filtered using a $0.22 \mu \mathrm{m}$ syringe filter. The filtered AuNPs was measured using a Cary 100 Bio UV-Vis spectrophotometer (Figure 48A), and the concentration was calculated by using Beer's law $(A=\varepsilon b c)\left(\varepsilon=2.7 \times 10^{-8} \mathrm{M}^{-1} \mathrm{~cm}^{-1}\right.$ at the maximum absorption at $519 \mathrm{~nm}$ ). The wavelength and shape of the LSPR peak provide the information regarding the size and shape of the AuNPs due to the total refractive index on the AuNP surface. ${ }^{231}$ An estimated theoretical diameter of $13 \mathrm{~nm}$ with a spherical shaped conformation can be attributed for our synthesized AuNPs. The characteristic bright red color of our synthesized AuNPs also indicates the nonaggregated form of the colloids that can irreversibly change color accompanied by a red shift upon aggregation. ${ }^{234}$ The homogeneity and size distribution of the synthesized AuNPs was estimated by measuring the hydrodynamic size and the polydispersity index (PDI) using dynamic light scattering (DLS) technique. ${ }^{235}$ The illuminated laser beam when passed through the suspension of AuNPs enables the DLS instrument to analyze the scattered light to obtain the velocity of particle's Brownian motion, thereby interpreting the hydrodynamic diameter of the AuNP to be $21 \mathrm{~nm}$ (Figure 48B) with a PDI of 0.1 , indicating a very narrow and uniform size distribution. The hydrodynamic diameter is larger than the actual diameter of the AuNPs as it reflects the core diameter of the 
nanoparticle along with the surface coating and the solvent layer associated with the particle. $^{236}$
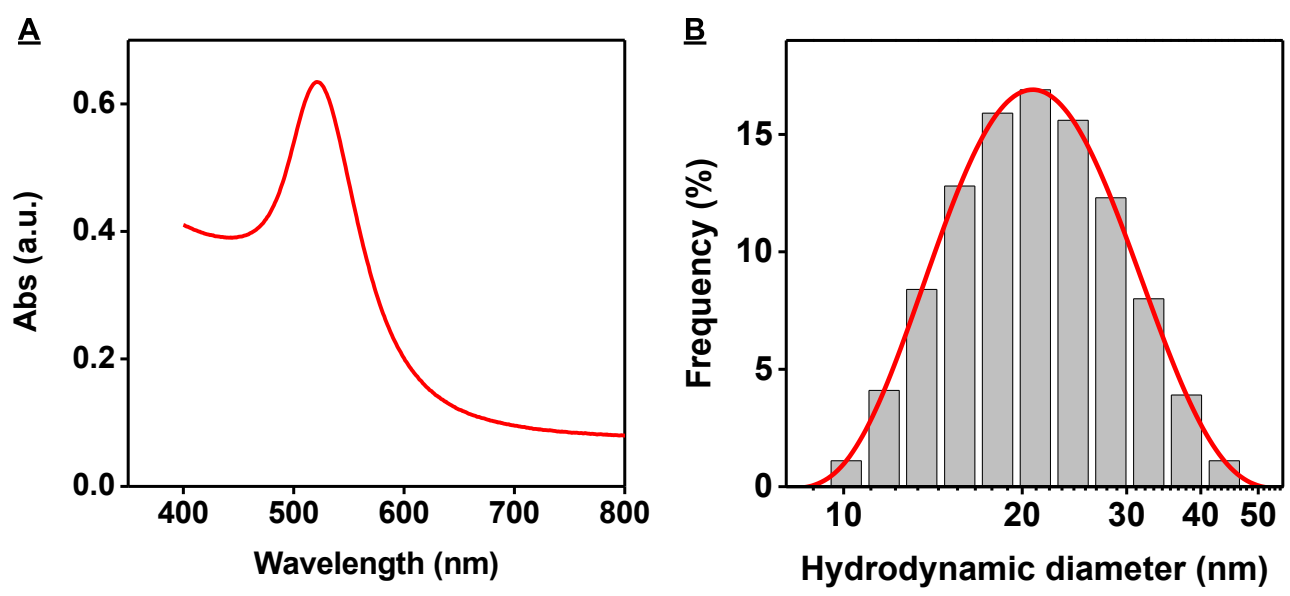

Figure 48. Concentration and dispersion of synthesized AuNPs. (A) UV-Vis spectrum of the synthesized AuNPs with a maximum wavelength of $519 \mathrm{~nm}$. (B) Particle size distribution measurement using dynamic light scattering showing the average hydrodynamic diameter of the AuNP to be $21 \mathrm{~nm}$, with polydispersity index (PDI) of 0.1 .

Though DLS technique gave us a rough estimate of the size of the synthesized AuNP, it is inconclusive about the structural morphology and physical size of the core. The actual shape and size of the AuNPs were characterized using Transmission Electron Microscopy (TEM). TEM images were obtained with a Phillip CM 200 microscope at $200 \mathrm{kV}$, where a tiny drop of AuNP suspension was placed on the copper grid and allowed to evaporate at room temperature. TEM image revealed a roughly spherical AuNPs appeared as small dots in the image (Figure 49A). Image analysis using Image $\mathrm{J}$ software, by taking the average of at least 200 AuNPs confirmed that the particles were uniform in size, with a diameter of $\sim 12 \pm 1 \mathrm{~nm}$ (Figure 49B). 


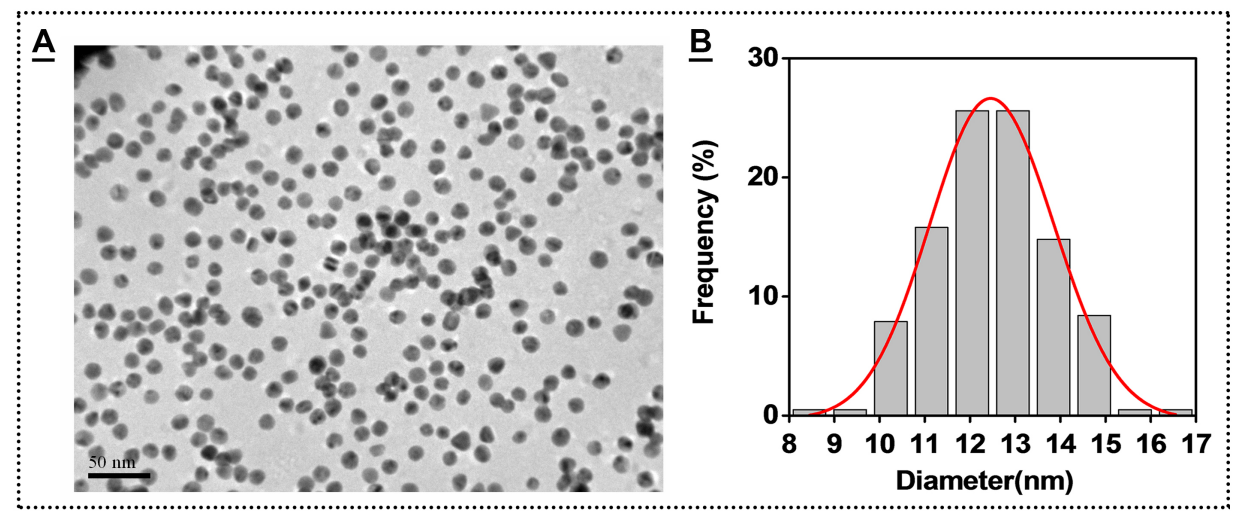

Figure 49. Transmission electron microscopy (TEM) image of (A) citrate-capped AuNPs using a Phillips CM-200 $200 \mathrm{kV}$ reveals similarly sized, roughly spherical particles. (B) Frequency distribution histogram shows an average particle diameter of $12 \pm 1-\mathrm{nm}$, as calculated using Image J software. 

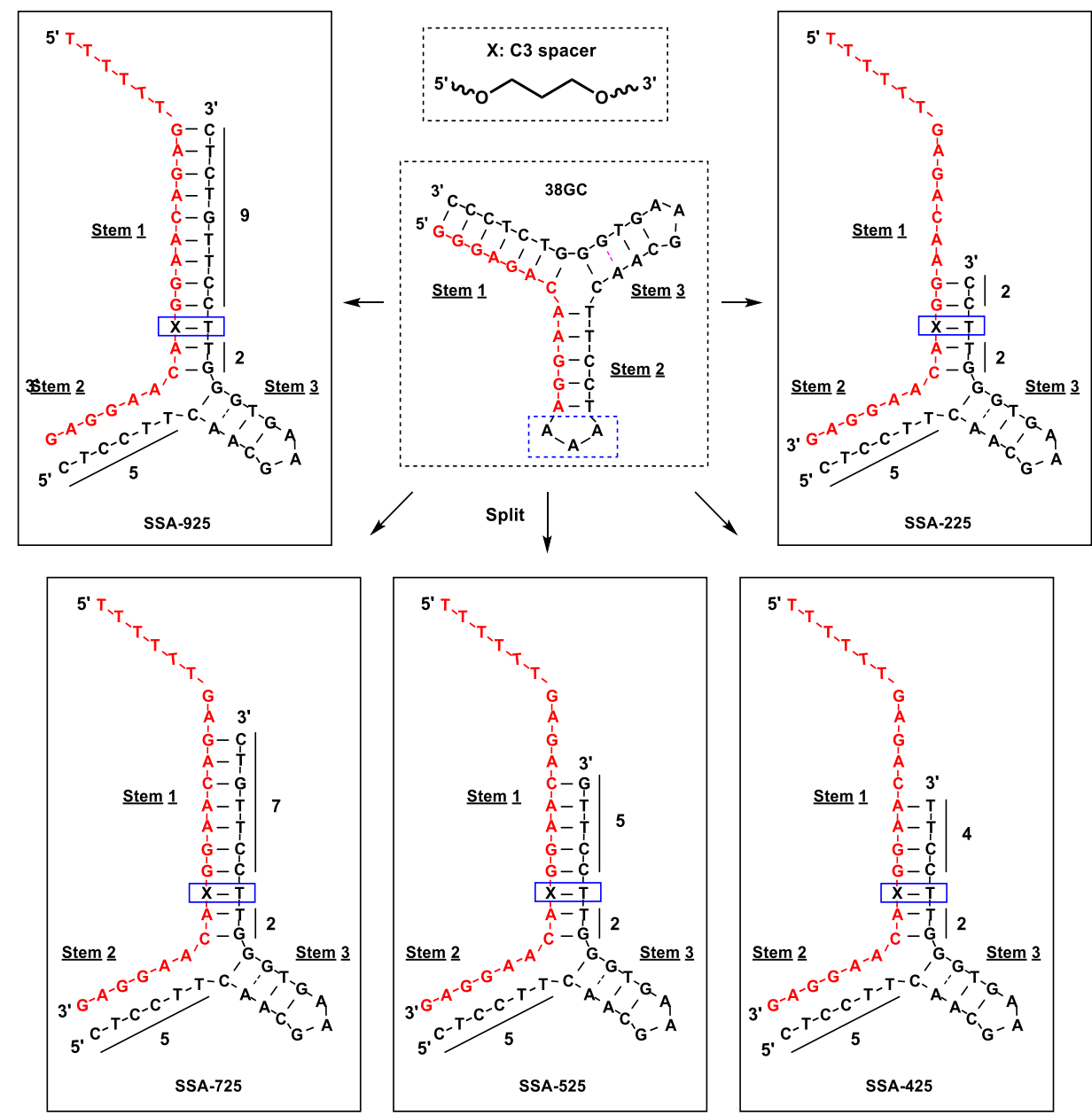

Figure 50. Formation of folded split aptamers with single binding domain (SSA) from its single cocaine-binding parent aptamer (38GC). These folded split aptamers assembled with cocaine are comprising of a short and long fragments.

Cocaine-binding aptamer (termed $38 \mathrm{GC})^{236}$ with a single binding domain developed from the original MNS-4.1, ${ }^{237}$ contained a three-way junction with the target-binding domain at its center, surrounded by three double-stranded stems (stems 1,2 and 3) and two loops (GAA and AAA) (Figure 50, 38GC). Previous studies identified the significance of stem 3 in cocaine binding, whereas stems 1 and 2 contribute for the overall thermal-stability of the target-aptamer complex in the event of target binding. ${ }^{236}$

To increase target-responded signal, this $38 \mathrm{GC}$ aptamer was split by removing the AAA loop and engineering several base-pairs in stem 1. Each split aptamer having a single 
binding domain (termed SSA-225, SSA-425, SSA-525, SSA-625, SSA-725, SSA-925) consists of a different long fragment (LF) and a shared short fragment (SF-SSA)(Figure 50). Note that the thermal-stability of SSA is increased with the increase of number of base pairs in stem 1. A C3 spacer was introduced in the short fragment to form an AP site (highlighted in the box) with a thymine in the opposite position within the long fragment upon cocaine binding (Figure 50). We found that this introduction of the AP site suggests an enhanced reaction speed through faster Exo III apurinic endonucleolytic digestion of the cocaine-aptamer complex. The thiolated SF-SSA was attached covalently onto the surface of AuNP by thiol-gold chemistry forming a self-assembled monolayer. We used various LF-SSA including LF-925, LF-725, LF-525, LF-425 and LF-225 with this SFSSA-modified AuNPs to demonstrate Exo III-assisted target recycling for cocaine detection.
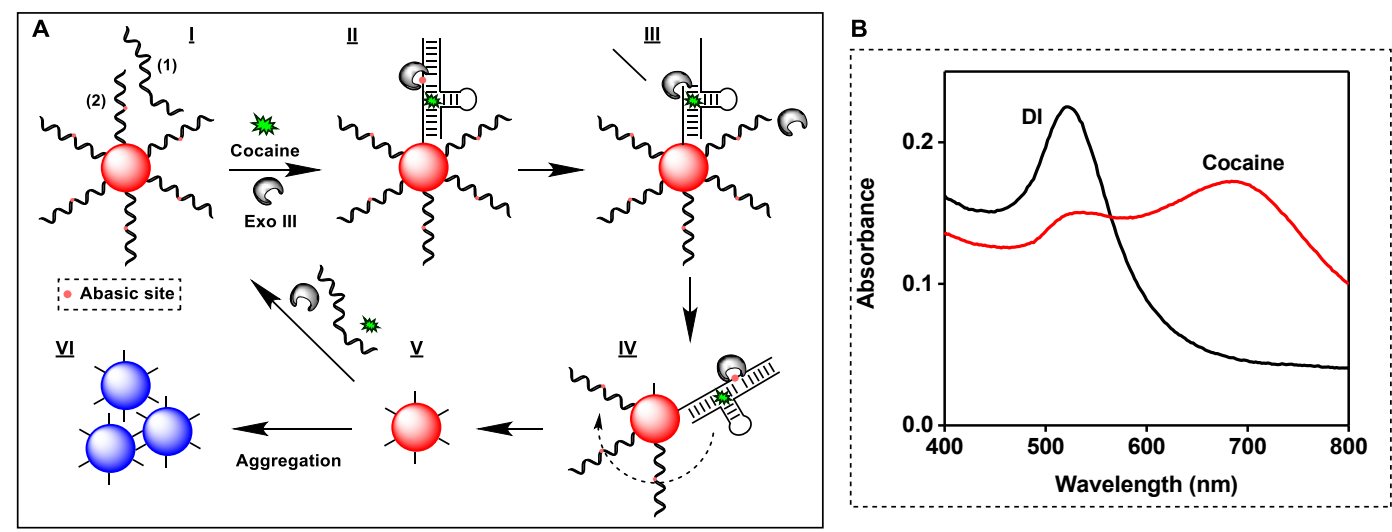

Figure 51. AuNP-reported, Exo III-assisted colorimetric detection of cocaine. (A) Schematic principle of the assay. (B) UV-vis spectra of the short fragment-modified AuNPs in the absence (black trace) and presence (red trace) of cocaine.

Non-specific adsorption of short aptamer fragment could occur during this detection, which limits the accessibility of the long aptamer fragment to form the complex. In order to prevent this, multiple thymine bases in the form of a spacer $\left(\operatorname{poly}(\mathrm{T})_{10}\right.$ spacer) were 
added to the short aptamer fragments (Figure 50). This poly $(\mathrm{T})_{10}$ spacer enables reducing non-specific adsorption due to their lower binding affinity towards gold surfaces as compared to other deoxynucleotides. ${ }^{238}$ Initially, the thiolated short fragment was modified on freshly synthesized AuNPs with it 5' thiol end attached to the surface. The aptamer modification on the surface of AuNP protects the particles from aggregation, even under high salt concentrations.(Figure 51A, I) Long fragment is then introduced into the reaction buffer and forms a target-aptamer complex in the presence of cocaine.(Figure 51A, II) After a 30-min incubation, Exo III was then added into the reaction mixture to initiate exonuclease cleavage process. The enzyme's apurinic endonucleolytic helps in cleaving the duplexed abasic site of the target-aptamer complex.(Figure 51A, III) Once the digestion of the short fragment is completed, the long fragment along with cocaine molecule are released into the solution that are available to assemble with another short fragment immobilized on the AuNP.(Figure 51A, IV) The digestion mechanism repeats until all the short aptamer fragments are cleaved from the surface of AuNPs (Figure 51A, V) leaving the surface unprotected therefore causing them to aggregate to form a red-toblue color change.(Figure 51A, VI and Figure 51B, with cocaine) In contrast, no targetaptamer complex is formed in the absence of cocaine, therefore inactivating the activity of Exo III. The sample still retains the red color of the AuNPs (Figure 51B, without cocaine). 

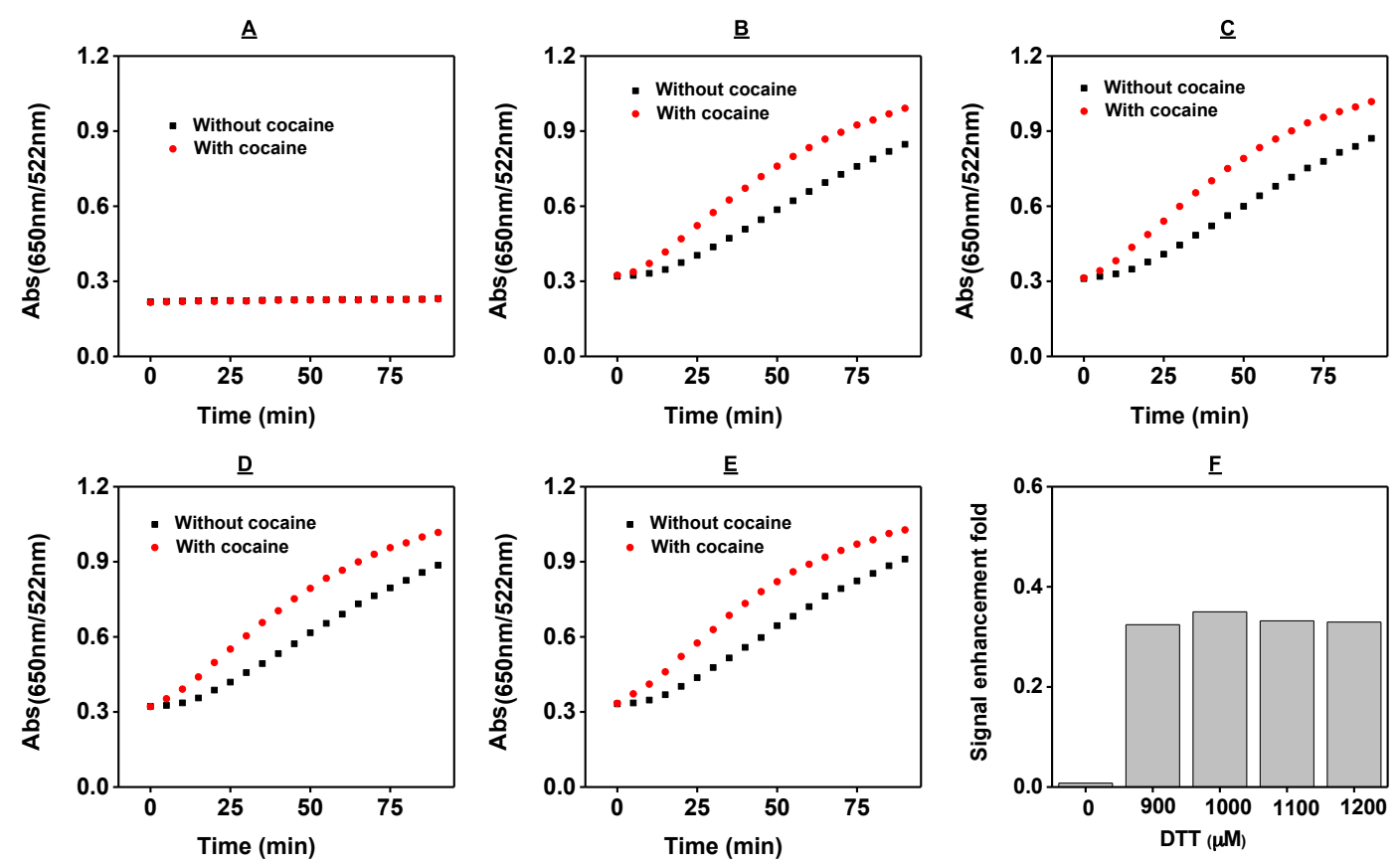

Figure 52. Effect of DTT concentration on the surface coverage of SF-SSA resulting in change of Exo III kinetics for cocaine detection. The SF-SSA-modified AuNPs were first treated with different concentration of DTT $(0-1200 \mu \mathrm{M})$; (A) $0 \mu \mathrm{M}$; (B) $900 \mu \mathrm{M}$; (C) $1000 \mu \mathrm{M}$; (D) $1100 \mu \mathrm{M}$; and (E) $1200 \mu \mathrm{M}$ to obtain different surface coverages. (F) Signal enhancement fold between cocaine and DI calculated with $\mathrm{A}_{650} / \mathrm{A}_{522}$ after 30 -min Exo III digestion.

The colorimetric detection of cocaine was performed in a final reaction volume of $25 \mu \mathrm{L}$ reaction buffer containing $10 \mathrm{mM}$ Tris buffer ( $\mathrm{pH}$ 7.4), with $100 \mathrm{mM} \mathrm{NaCl}, 1 \mathrm{mM}$ $\mathrm{MgCl}_{2}$, optimized concentration of long aptamer fragment, $100 \mathrm{nM}$ SF-SSA-modified AuNPs and $20 \mathrm{U}$ Exo III, in the presence and absence of cocaine $(250 \mu \mathrm{M})$ giving 30 minutes to form the aptamer-target complex, at room temperature before adding the Exonuclease III. The change in the color was characterized by measuring the UV-Vis spectra of both the samples with and without cocaine over time, and plotted against each other in-terms of absorbance ratio of $\mathrm{A}_{650} / \mathrm{A}_{522}$ (Figure 52). The efficiency of forming the target-aptamer complex and the kinetics of Exo III digestion are greatly affected by stability of the target-aptamer complex, density of SF-SSA on the surface of AuNPs, and 
concentration of long aptamer fragment. As mentioned before the aptamer density on the surface of AuNP plays a crucial role as it affects the target-aptamer binding process with the long aptamer fragment and cocaine, thereby affecting the specificity, and reaction time. Thus, we used different concentrations of DTT to treat our SF-SSA-modified AuNPs and perform the colorimetric detection of cocaine at each of those concentrations. To obtain the optimal concentration of DTT that would ultimately result in higher targetassembly efficiency, we first incubated different concentrations of DTT with SF-SSAmodified AuNPs for 30 minutes at room temperature. During this incubation step, the SFSSA was displaced from the surface of AuNPs proportional to the concentration of DTT to yield different DNA surface coverages. The DTT-treated SF-SSA-modified AuNPs were initially mixed with the long fragment of 525 (LF-525) (100nM as final concentration) that was able to form five complementary bases above the inserted AP site with AuNP-conjugated SF-SSA in the presence of cocaine. Cocaine $(250 \mu \mathrm{M})$ was added into half of above reaction mixture whereas deionized water (DI) was added to the other half. Both samples were incubated at room temperature for 30 minutes in a microtitre plate. Exo III enzyme was then added to all wells to record the change in absorption ratio $\left(\mathrm{A}_{650} / \mathrm{A}_{522}\right)$ for each set of DTT concentration along with the control set without DTT treatment (Figure 52).

We observed that the SF-SSA-modified AuNPs without DTT treatment resulted in slower kinetics for Exo digestion with a poor color differentiation between the samples with and without cocaine. Both samples were red in color even after several hours of Exo reaction as evident in Figure 52A. As the DTT concentrations increased from $900 \mu \mathrm{M}$ to 1200 $\mu \mathrm{M}$, we observed the change in reaction rate evident from the digestion kinetics of Exo 
III (Figure 52, B to E). Although the rate of Exo digestion was visualized in the form of a color change from red to light purple in both samples, the signal enhancement fold of $\mathrm{A}_{650} / \mathrm{A}_{522}$ between cocaine and DI after 30-min of Exo III digestion was still below 0.6 which is used as a threshold to identify distinguished color change in cocaine sample. The values of $\mathrm{A}_{650} / \mathrm{A}_{522}$ on the $\mathrm{y}$-axis of the plots represent the aggregation state of the AuNPs and the starting point on the y-axis of each plot represents the aggregation state of the DTT-treated AuNPs irrespective of the presence or absence of cocaine. At lower DTT concentrations such as $900 \mu \mathrm{M}$, the probe density on the AuNPs is too high therefore limiting the binding efficiency of the aptamer fragments with the target, and preventing the specific enzyme digestion. However, very high DTT concentrations such as $1200 \mu \mathrm{M}$ resulted in very few aptamer fragments remained on the surface, indicated by an aggregated state even before addition of Exo III, therefore unfavorable for sensitive colorimetric detection. Thus we chose an optimum DTT concentration of $1000 \mu \mathrm{M}$, which left enough SF-SSA fragments on the surface of the AuNP for remaining nonaggregated state and facilitating high target-binding efficiency and faster enzyme kinetics in the presence of cocaine compared to its absence (Figure 52F).
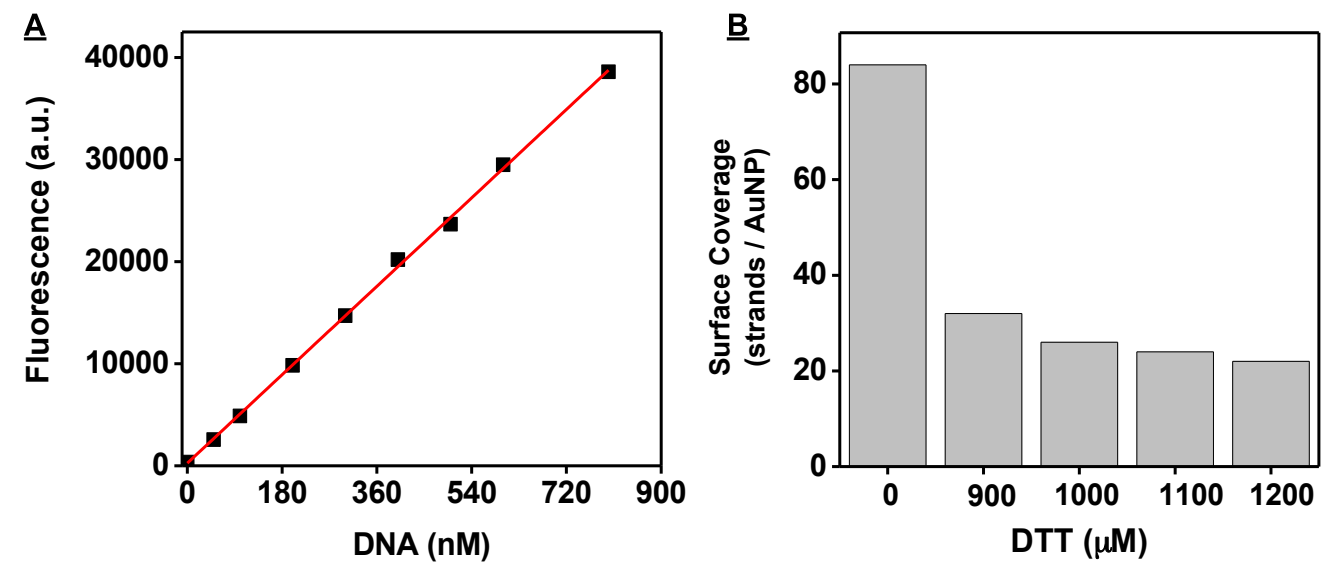
Figure 53. Calculation of surface coverage of SF-SSA-modified AuNPs treated with different concentrations of DTT from $0 \mu \mathrm{M}$ to $1200 \mu \mathrm{M}$ using Oligreen assay (QuantiT ${ }^{\mathrm{TM}}$ OliGreen ${ }^{\circledR}$ ssDNA Reagent) (A) Calibration curve based on fluorescence, (B) Calculation of DNA strands per AuNP at different DTT concentrations.

To identify the number of SF-SSA fragments on the AuNP at DTT concentration of 1000 $\mu \mathrm{M}$, we have performed Oligreen assay using Quant-iT ${ }^{\mathrm{TM}}$ OliGreen ${ }^{\circledR}$ ssDNA Reagent (Figure 53). We used a standard curve generated from unconjugated, thiolated SF-SSA with DTT under the same experimental conditions (Figure 53A) to calculate aptamer surface coverage. We determined that SF-SSA-modified AuNPs treated with concentration of DTT of $0,900,1000,1100$, and $1200 \mu \mathrm{M}$ respectively displayed $84 \pm 6$, $38 \pm 4,30 \pm 3,29 \pm 2$, and $26 \pm 2$ oligonucleotides per particle (Figure 53B), equivalent to surface coverage of $26.3 \pm 1.3,11.9 \pm 1.1,9.4 \pm 0.8,9.1 \pm 0.6$, and $8.1 \pm 0.6 \mathrm{pmole} / \mathrm{cm}^{2}$, respectively. Our experimental results demonstrated that 30 SF-SSA strands per AuNP at $1000 \mu \mathrm{M}$ DTT concentration was optimum surface coverage required to give a color change specific to the presence of cocaine after Exo III digestion. However, the time required for the sample with cocaine to turn blue is about $50 \mathrm{~min}$, which is too long for an onsite drug screening. Additionally, when the cocaine sample changed color from red to blue after a 50-min Exo III digestion, the control sample without cocaine also changed to purple therefore making it hard to differentiate between the colors.

As mentioned earlier one of the variables to improve the cocaine-assembly efficiency is the availability of the target-aptamer complexes. The concentration of LF thus plays a crucial role, and need to be optimized to achieve a high signal enhancement fold. Therefore, we tested the effect of LF-525 concentrations on the cocaine-assembled Exo III digestion (Figure 54). As expected, we observed no color difference between the samples with and without cocaine when there is no LF-525 existed in the solution, 
evidently due to the lack of formation of target-aptamer complex. This phenomenon is also true for the lowest LF-525 concentration of $50 \mathrm{nM}$ because the insufficient amount of LF-525 was able to use for cocaine specific binding. At higher concentrations of LF525 such as $1 \mu \mathrm{M}$ or $5 \mu \mathrm{M}$, more target-aptamer complexes were formed in both cocaine and DI samples and the enzymatic reactions seem faster. However, the signal enhancement fold of $\mathrm{A}_{650} / \mathrm{A}_{522}$ between cocaine and DI after 30-min of Exo III digestion was decrease. We found that $100 \mathrm{nM} \mathrm{LF-525}$ gave the highest signal enhancement fold of 0.35 and optimum color difference between the samples with and without cocaine (Figure 54F). However, the specificity still remains a problem along with no improvement in the reaction time.

The length of stem 1 on the long fragment usually contributes to thermal-stability of cocaine-aptamer complex. It is known that long stems may result in poor specificity due to pre-assembly of target-aptamer complexes even in the absence of cocaine, whereas short stems may not enable formation of aptamer-target complexes in the presence of cocaine due to poor thermal-stability. In an attempt to improve the specific formation of the target-aptamer complex in the presence of cocaine, we studied the effect of different long fragment's stem lengths on target-aptamer assembly and Exo digestion. Specifically, we used LF-525 to respectively elongated two or four base pairs at its 3' end to form LF725 and LF-925, or truncated one or three base pairs from the 3' end of LF-525 to engineer LF-425 and LF-225, respectively. 

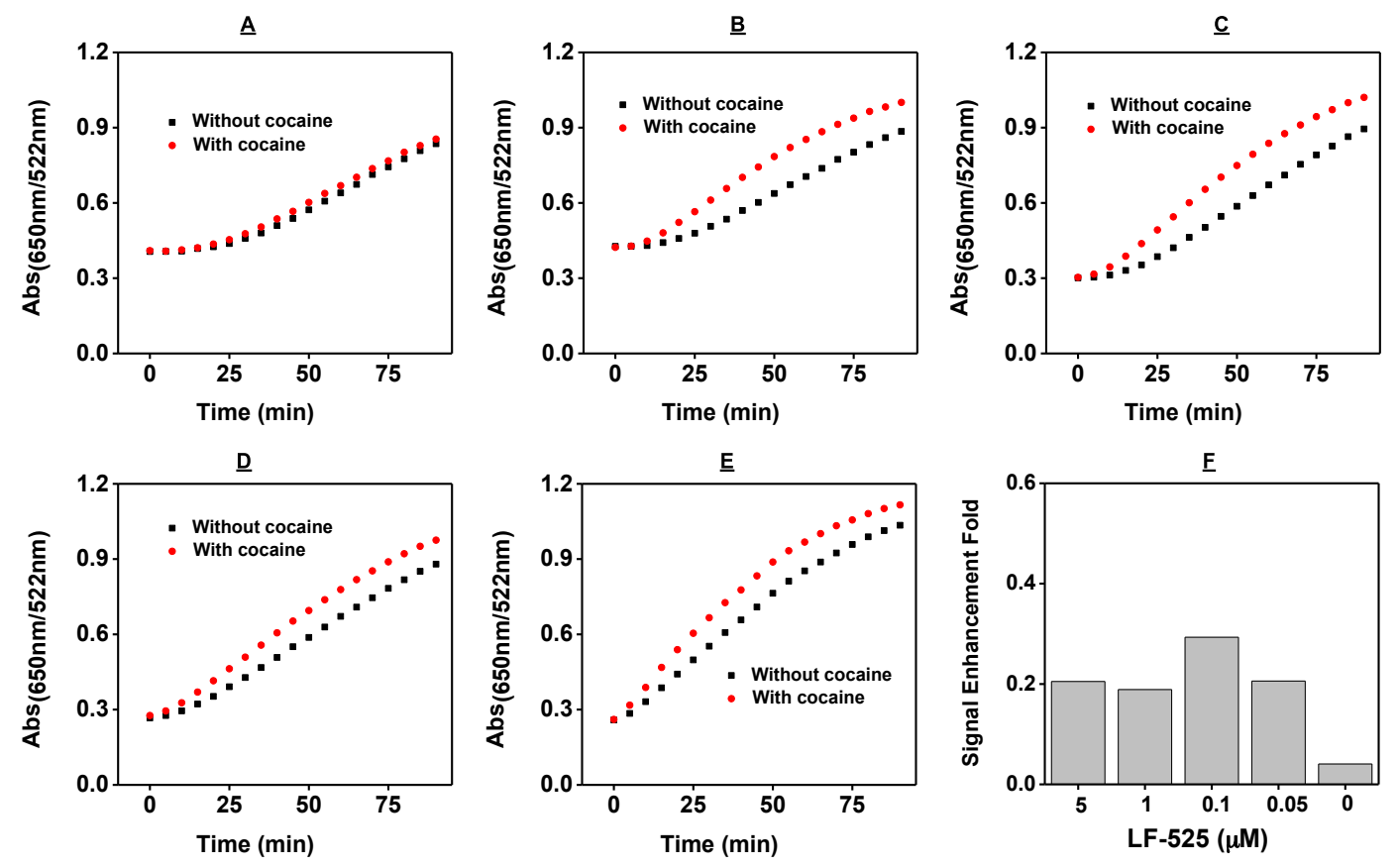

Figure 54. Effect of the LF-525 concentration on the efficiency of target-aptamer assembly and Exo III kinetics for cocaine detection. The SF-SSA-modified AuNPs were first treated with optimized DTT concentration of $1000 \mu \mathrm{M}$ to obtain the surface coverage of 30 DNA strands per particle, to which variable concentrations of LF-525 were added ranging from 5 to $0 \mu \mathrm{M}$. (A) $0 \mu \mathrm{M}$; (B) $0.05 \mu \mathrm{M}$; (C) $0.1 \mu \mathrm{M}$; (D) $1 \mu \mathrm{M}$; (E) $5 \mu \mathrm{M}$. (F) Signal enhancement fold between cocaine and DI calculated with $\mathrm{A}_{650} / \mathrm{A}_{522}$ after 30 -min Exo III digestion. 

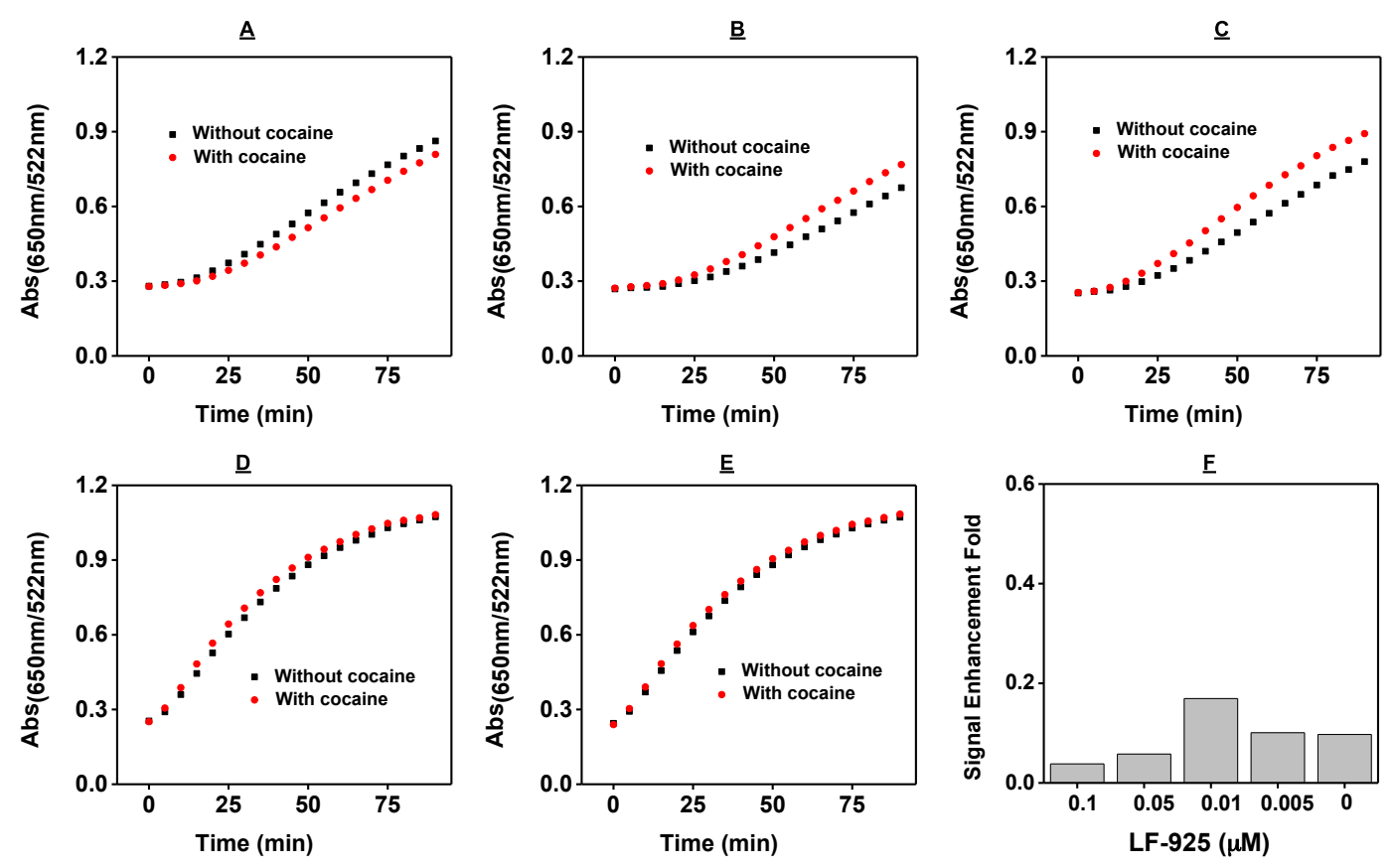

Figure 55. Effect of LF-925 concentration on the efficiency of target-aptamer assembly and Exo III kinetics for cocaine detection. The SF-SSA-modified AuNPs were first treated with optimized DTT concentration of $1000 \mu \mathrm{M}$ to obtain the surface coverage of 30 DNA strands per AuNP, to which variable concentrations of LF-925 DNA were added ranging from $100 \mathrm{nM}$ to $0 \mathrm{nM}$. (A) $0 \mathrm{nM}$; (B) $5 \mathrm{nM}$; (C) $10 \mathrm{nM}$; (D) $50 \mathrm{nM}$; and (E) 100 nM. (F) Signal enhancement fold between cocaine and DI calculated with $\mathrm{A}_{650} / \mathrm{A}_{522}$ after 30-min Exo III digestion.

Note that all these LFs bind a common AuNP-conjugated SF-SSA to form a single AP site and a single binding domain in the presence of cocaine. To investigate the effect of different LFs on target-aptamer assembly and Exo digestion, we have fixed the number of SF-SSA on the AuNP by fixing the optimized DTT concentration and changed the concentration of the LF with variable lengths in order to find out the best LF length combined with optimized concentration that gives the highest signal enchancement fold after a 30-min Exo reaction. Therefore, the optimized probe density of 30 DNA strands / AuNP was used to test the effect of LF-925 concentration on target-aptamer assembly and Exo digestion. Although we observed faster enzyme reaction in the samples with and without cocaine for all tested LF-925 concentrations, the specificity was poor due to 
higher stability of the complex resulted by the longer stem 1 (Figure 55, A, B, C, D and E). As expected, a small LF concentration was required for LF-925 and a $10 \mathrm{nM} \mathrm{LF-925}$ gave the highest signal enhancement fold of 0.18 (Figure 55F).

From the experimental results obtained with LF-925, it is evident that the two aptamer fragments were assembled even in the absence of target due to a high thermo-stability. We then used the optimized probe density of 30 DNA strands/AuNP to test the effect of LF-725 concentration on target-aptamer assembly and Exo digestion. We observed faster enzyme reaction in the samples with and without cocaine, and a slight improvement of the specificity with Ls-725 was obtained (Figure 56, A, B, C, D and E). We found that a $50 \mathrm{nM}$ LF-725 gave the highest signal enhancement fold of 0.4 (Figure 56F).
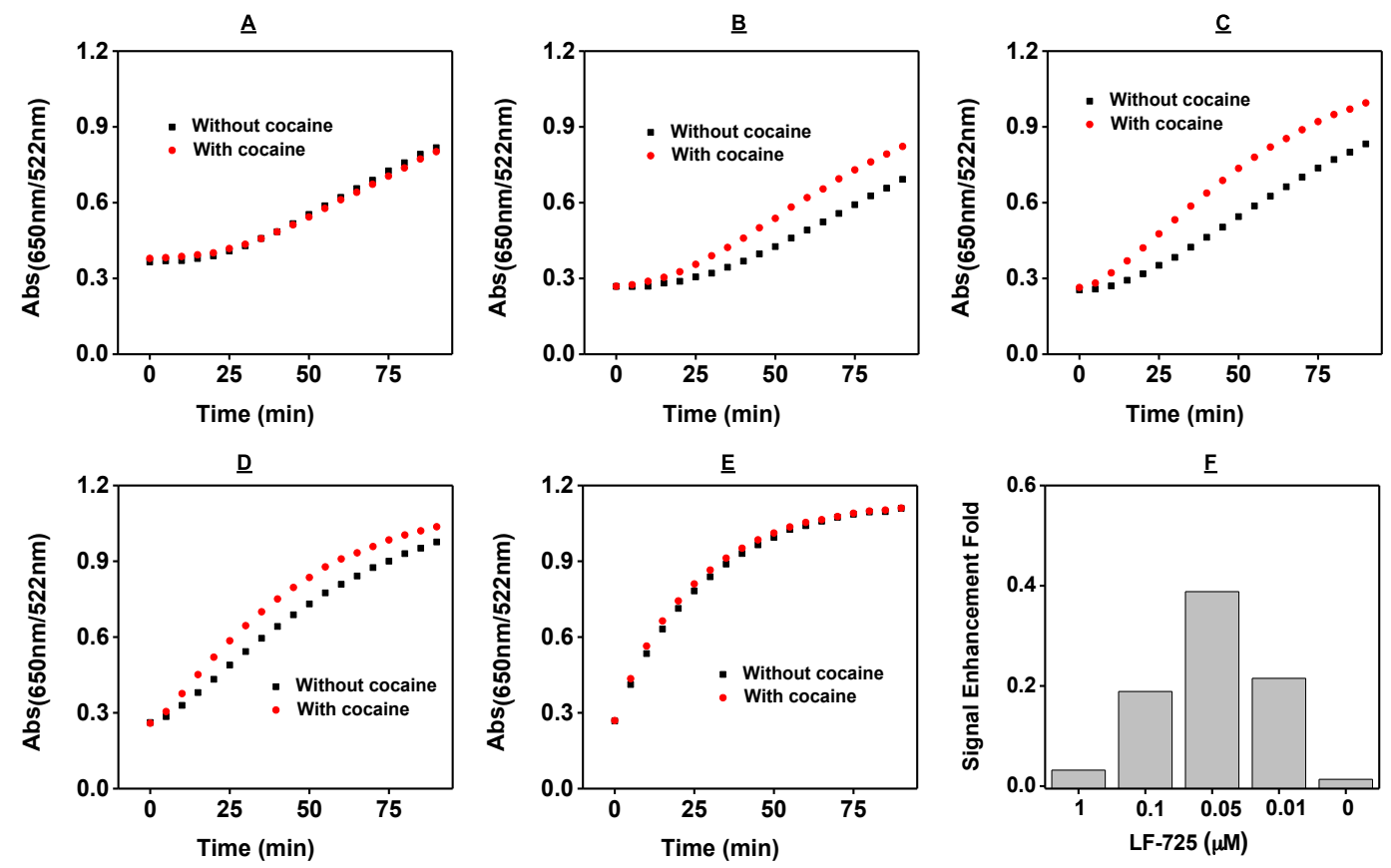

Figure 56. Effect of LF-725 concentration on the efficiency of target-aptamer assembly and Exo III kinetics for cocaine detection. The SF-SSA-modified AuNPs were first treated with optimized DTT concentration of $1000 \mu \mathrm{M}$ to obtain the surface coverage of 30 DNA strands per AuNP, to which variable concentrations of LF-725 DNA were added ranging from $1 \mu \mathrm{M}$ to $0 \mathrm{nM}$. (A) $0 \mu \mathrm{M}$; (B) $0.01 \mu \mathrm{M}$; (C) $0.05 \mu \mathrm{M}$; (D) $0.1 \mu \mathrm{M}$; and (E) 1 nM. (F) Signal enhancement fold between cocaine and DI calculated with $A_{650} / A_{522}$ after 30-min Exo III digestion. 

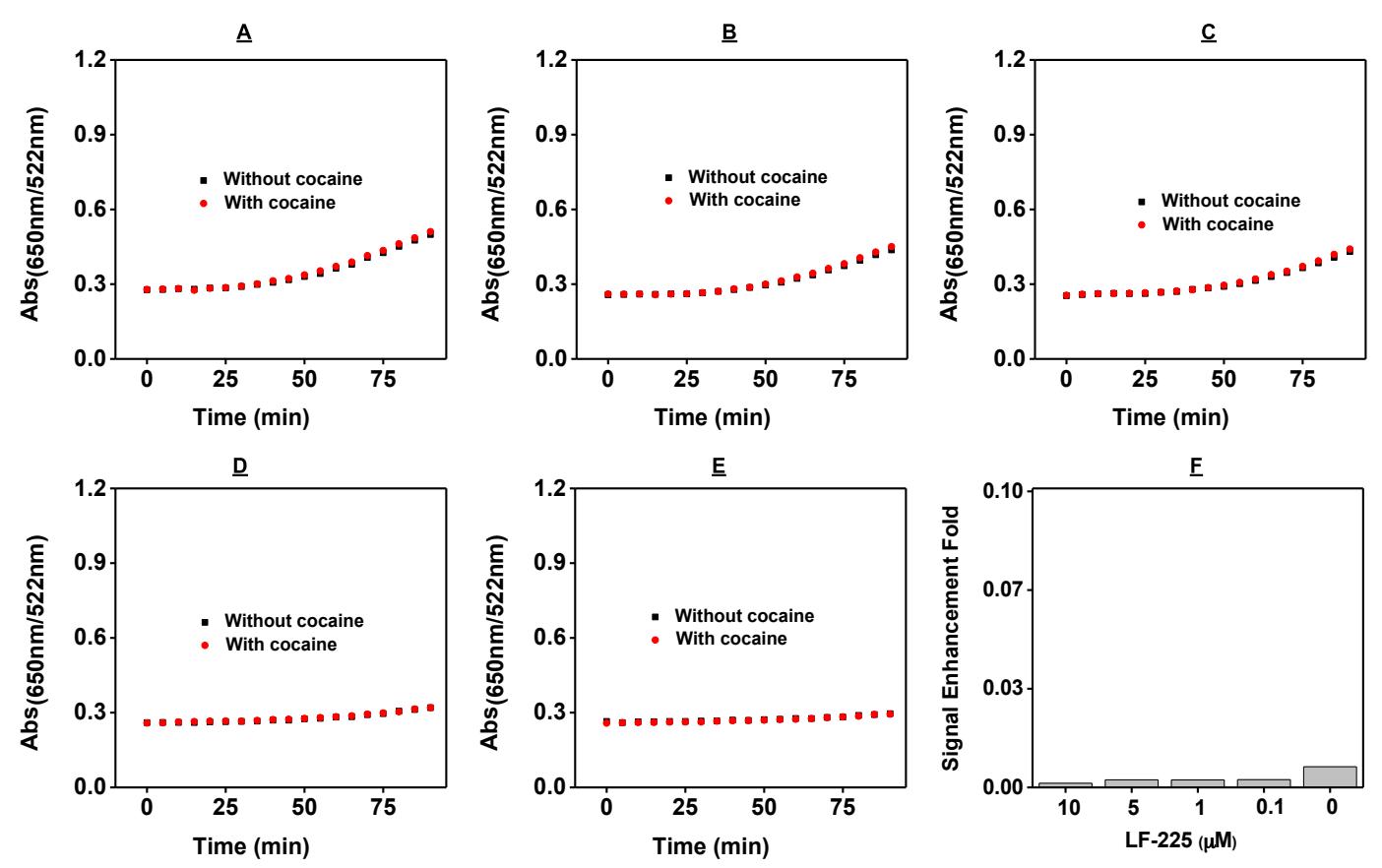

Figure 57. Effect of LF-225 concentration on the efficiency of target-aptamer assembly and Exo III kinetics for cocaine detection. The SF-SSA-modified AuNPs were first treated with optimized DTT concentration of $1000 \mu \mathrm{M}$ to obtain the surface coverage of 30 DNA strands per AuNP, to which variable concentrations of LF-225 DNA were added ranging from $10 \mu \mathrm{M}$ to $0 \mathrm{nM}$. (A) $0 \mu \mathrm{M}$; (B) $0.1 \mu \mathrm{M}$; (C) $1 \mu \mathrm{M}$; (D) $5 \mu \mathrm{M}$; and (E) 10 nM. (F) Signal enhancement fold between cocaine and DI calculated with $\mathrm{A}_{650} / \mathrm{A}_{522}$ after 30-min Exo III digestion.

A short stem length of LF would destabilize the aptamer complex in the absence of cocaine, thereby greatly decreasing background assembly. We further tested the effect of concentration of short LF such as LF-425 and LF-225 on target-aptamer assembly and Exo digestion. When LF-225 was added in the concentration range of $0 \mu \mathrm{M}$ to $10 \mathrm{nM}$, the Exo reaction was the slowest for all employed concentrations due lower stability of the target-aptamer complex and no specificty was observed between cocaine sample and DI sample (Figure 57). When the LF-425 was used for cocaine assembly and Exo digestion, we observed similar reaction kinetics as that of LF-225, with the complex formation between the AuNP-conjugated SF-SSA and LF-425 without much improvement specificity for the presence or absence of cocaine (Figure 58). 
Clearly, LF-725 showed a slight improvement of specificity over all other LFs we tested. However, the difference was not significant to observe a distinguished color change in the presence of cocaine.
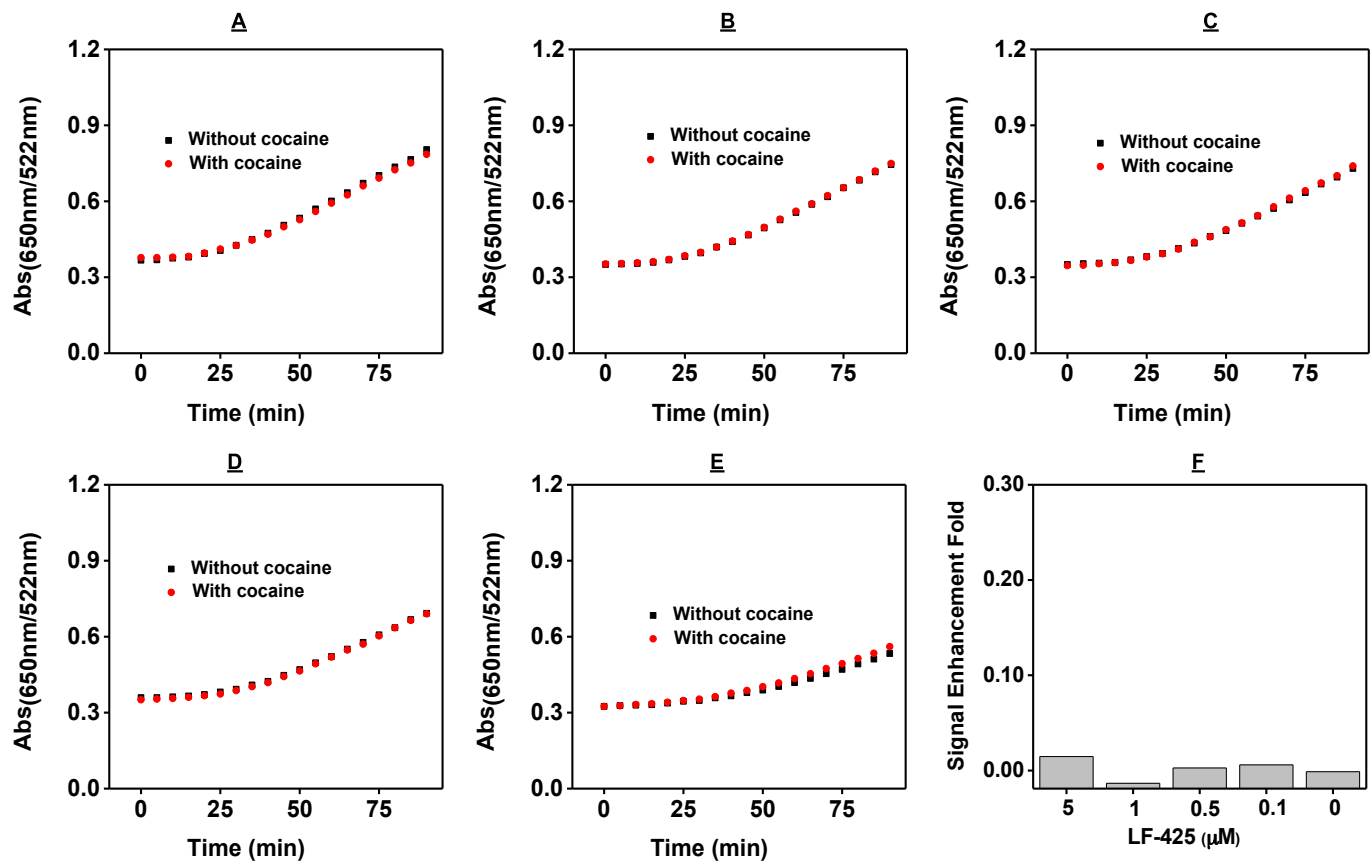

Figure 58. Effect of LF-425 concentration on the efficiency of target-aptamer assembly and Exo III kinetics for cocaine detection. The SF-SSA-modified AuNPs were first treated with optimized DTT concentration of $1000 \mu \mathrm{M}$ to obtain the surface coverage of 30 DNA strands per AuNP, to which variable concentrations of LF-425 DNA were added ranging from $5 \mu \mathrm{M}$ to $0 \mathrm{nM}$. (A) $5 \mu \mathrm{M}$; (B) $1 \mu \mathrm{M}$; (C) $0.5 \mu \mathrm{M}$; (D) $0.1 \mu \mathrm{M}$; and (E) $0 \mathrm{nM}$. (F) Signal enhancement fold between cocaine and DI calculated with $\mathrm{A}_{650} / \mathrm{A}_{522}$ after 30min Exo III digestion.

To achieve more specific and switch-like binding of the aptamer to cocaine target, we introduced a second target-binding domain into our SSA with a single target-binding domain and studied new aptamer sequence affect on formation of cocaine-aptamer complex. We confirmed that the binding of aptamer to cocaine target at the first binding domain stabilizes the structure of the aptamer, facilitating the cocaine binding at the second binding domain. ${ }^{156}$ This kind of phenomenon called cooperative behavior was 
first observed in ligand-binding proteins that are highly responsive to ligand concentration, such as hemoglobin. ${ }^{149}$

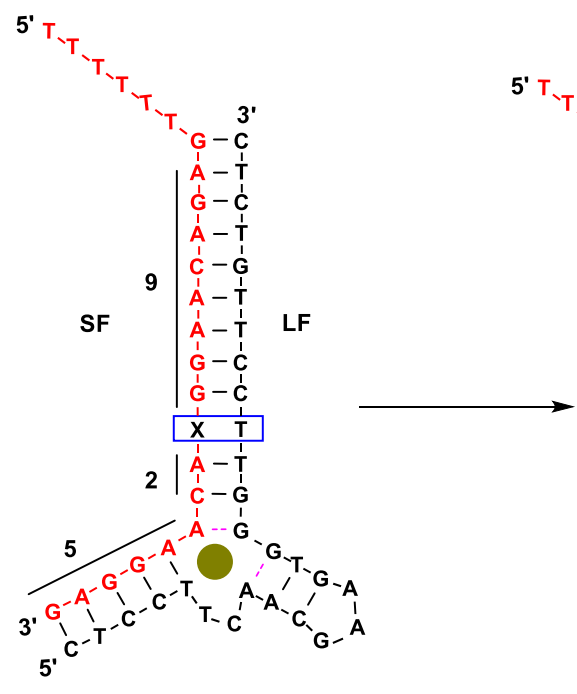

SSA-925

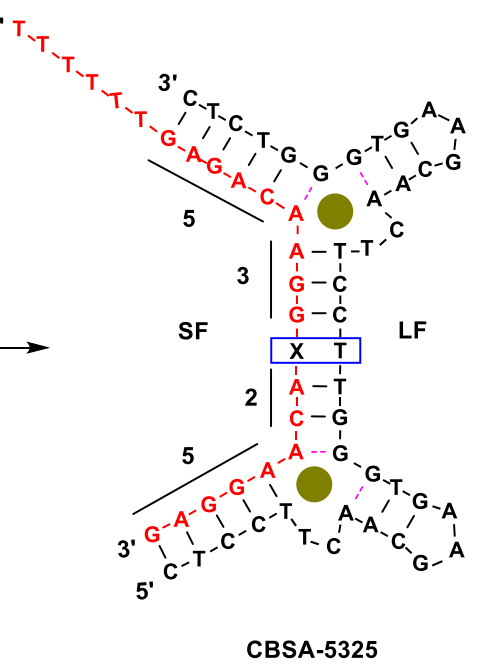

CBSA-5325

Figure 59. Utilizing SSA-925 to derivate CBSA-5325. Both structures demonstrated cocaine-bound status.

Our SSA contains a three-way junction target-binding domain, surrounded by three double-stranded stems (stems 1, 2 and 3). We have determined that stem 3 is essential for cocaine binding, while both stem 1 and stem 2 contribute to the stability of the three-way junction structure that forms upon target binding. ${ }^{60}$ We derived a cooperative binding split aptamer (CBSA) utilizing SSA-925. Specifically, we replaced thymine at position 6 from 3' end with the intact stem 3 to form CBSA-5325 (Figure 59). 

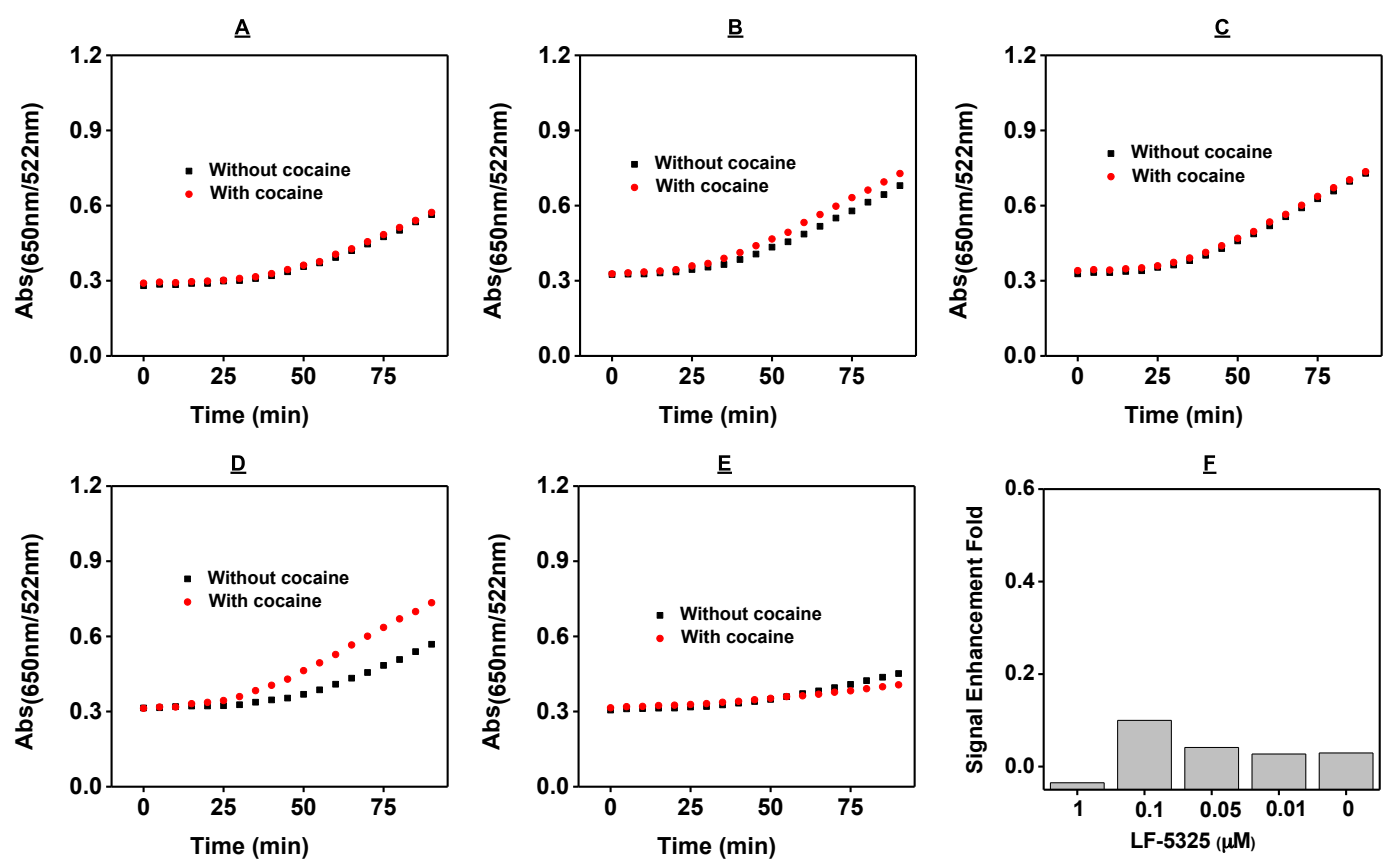

Figure 60. Effect of LF-CBSA-5325 concentration on the efficiency of target-aptamer assembly and Exo III kinetics for cocaine detection. The SF-SSA-modified AuNPs were first treated with optimized DTT concentration of $1000 \mu \mathrm{M}$ to obtain the surface coverage of 30 DNA strands per AuNP, to which variable concentrations of LF-CBSA-5325 were added ranging from $1 \mu \mathrm{M}$ to $0 \mathrm{nM}$. (A) $0 \mu \mathrm{M}$; (B) $0.01 \mu \mathrm{M}$; (C) $0.05 \mu \mathrm{M}$; (D) $0.1 \mu \mathrm{M}$; and (E) $1 \mathrm{nM}$. (F) Signal enhancement fold between cocaine and DI calculated with $\mathrm{A}_{650} / \mathrm{A}_{522}$ after 30-min Exo III digestion.

The resulting CBSA also consists of a short fragment (SF-SSA) and a long fragment (LFCBSA-5325). Note that LF-CBSA-5325 can utilize the same AuNP-conjugated SF-SSA to form target-aptamer complex in the presence of cocaine. Because the AP site is inserted between two binding domains, we assume that Exo III is able to recognize the duplexed AP site and perform its endonucleolytic cleavage. To confirm this, we first tested the digestion of Exo III using the LF-CBSA-5325 to confirm that the cocaineCBSA assembly is a preferred substrate for Exo III. Specifically, the colorimetric detection of cocaine was performed in a final reaction volume of $25 \mu \mathrm{L}$ reaction buffer containing $10 \mathrm{mM}$ Tris buffer (pH 7.4), with $100 \mathrm{mM} \mathrm{NaCl}, 1 \mathrm{mM} \mathrm{MgCl}$, various concentrations of LF-CBSA-5325, 100 nM SF-SSA-modified AuNPs with optimized 
probe density and $20 \mathrm{U}$ Exo III, in the presence and absence of cocaine $(250 \mu \mathrm{M})$ at room temperature. Experimental results indicated that the CBSA-5325 system did not show much improvement in the specificity of the reaction, however further slowed down the reaction speed primarily due to the formation of destabilized cocaine-CBSA complexes (Figure 60, A, B, C, D and E). Highest signal enhancement fold of 0.13 was observed with $100 \mathrm{nM}$ LF-CBSA-5325 (Figure 60B, 13F).

There is very poor color distinction between the samples with and without cocaine when the concentration of the LF-CBSA-5325 increased or decreased from $100 \mathrm{nM}$ (Figure $60 \mathrm{~F})$. The poor digestion kinetics observed as a slow color change (Figure 60A) when the concentration of the LF-CBSA-5325 is $1 \mu \mathrm{M}$, which may be attributed to the limited number of duplex formed on the particle surface due to excessive long fragments causing steric hindrance. On the other hand, reducing the LF-CBSA-5325 concentration to $50 \mathrm{nM}$ gave an increased digestion kinetics, however with poor specificity (Figure 60C). Further decreasing the LF-CBSA-5325 concentration to $10 \mathrm{nM}$ did not yield an improved specificity without distinctive color change (Figure 60F) possibly due to the scarcity of the cocaine-specific duplex formation at lower LF concentration (Figure 60D). As expected, the signal enhancement fold of 0.04 (Figure 60F) obtained in the absence of the long fragment is similar to the fold observed with $10 \mathrm{nM} \mathrm{LF-CBSA-5325}$ due to the nonspecific Exo digestion (Figure 60E). 

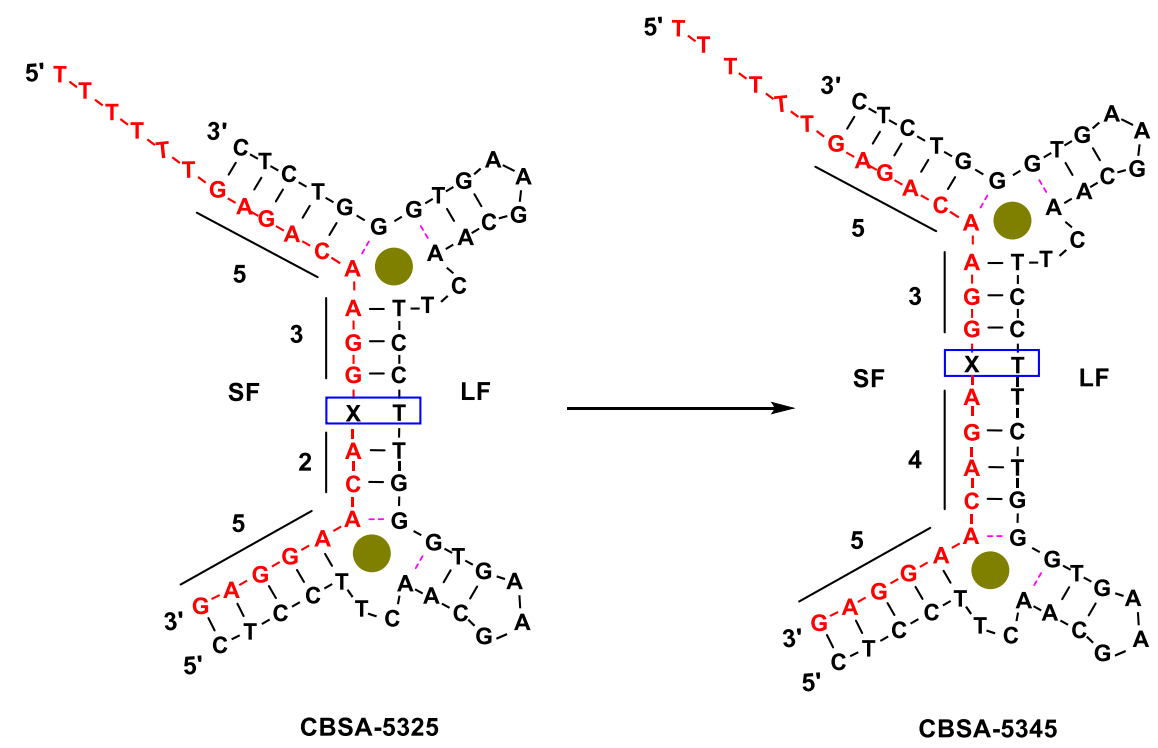

Figure 61. Utilizing CBSA-5325 to engineer CBSA-5345. CBSA Structure of folded split cocaine aptamer with dual binding domain assembled in the presence of cocaine comprising of short fragment and a long fragment.

To improve the stability and specificity of the cocaine-CBSA complex, we converted CBSA-5325 into CBSA-5345 by adding two new base pairs between two binding domain (Figure 61). Specifically, two additional bases (A, G) were added after the AP site of LFCBSA-5325, towards the 3' end of the sequence. Thus, the introduction of these two bases will increase the length between two binding domains from five bases in CBSA5325 to seven bases in CBSA-5345. This increased length may greatly improve the stability of cocaine-CBSA complex as observed in case of SSA-725 but with additional benefit of cooperative target-binding behavior. The long fragment LF-CBSA-5325 is also modified by the addition of two complementary bases $\mathrm{T}$ and $\mathrm{C}$ to hybrid the added $\mathrm{A}$, and G bases in SF-CBSA-5345, will therefore be referred to as LF-CBSA-5345.

Other CBSA aptamer (SF-CBSA-5335) was engineered from the SF-CBSA-5325 apart from SF-CBSA-5345. This modified aptamer added one additional base (G) between two binding domain (Figure 62), towards the 3' end of the short sequence. The addition of an 
extra base in SF-CBSA-5335 increased the length between two binding domain and improved the stability of the cocaine-CBSA complex. The overall thermo stability of the duplex for easy access of Exonuclease to the AP site may be improved when the binding domains are equidistant from the AP site on either side rather than variable lengths in SFCBSA-5345 and SF-CBSA-5325. The presence of one less base in SF-CBSA-5335 compared to SF-CBSA-5345 will ensure faster dissociation of the complex once the cocaine-CBSA complex is digested. The long fragment complementary to the SF-CBSA5335 will be modified from the existing LF-CBSA- 5325 by adding a complementary C base to the added G base in SF-CBSA-5335 and therefore will be referred to as LFCBSA-5335.

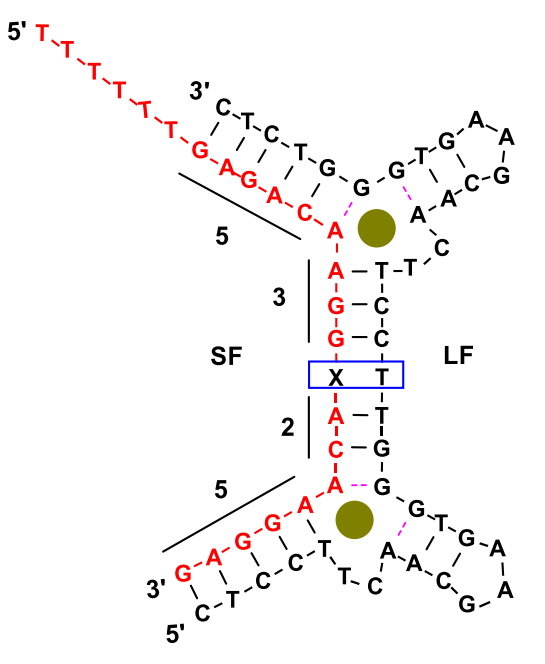

CBSA-5325

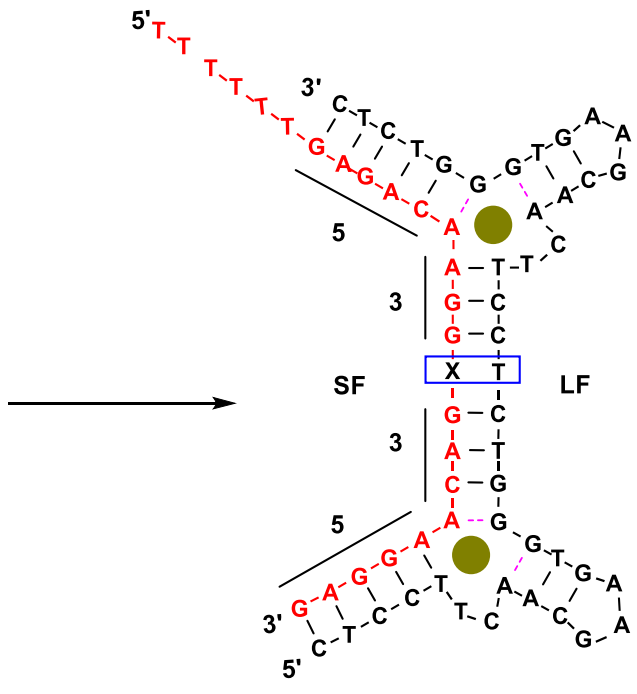

CBSA-5335

Figure 62. Utilizing CBSA-5325 to engineer CBSA-5335. CBSA Structure of folded split cocaine aptamer with dual binding domain assembled in the presence of cocaine comprising of short and a long fragments.

To optimize the SF-CBSA density on the AuNP surface for maximum target-CBSA binding with the LF-CBSA in presence of cocaine we used different concentrations of DTT to achieve maximum signal enhancement fold with a rapid reaction time. DTT 
incubation assay was performed on the SF-CBSA-5345 modified AuNPs, the same way as described above. As the DTT concentrations increased from $0 \mu \mathrm{M}$ to $400 \mu \mathrm{M}$, we observed the change in reaction rate relative to the digestion kinetics of Exo III (Figure 63, B to E). Although the rate of Exo digestion was visualized in the form of a color change from red to light purple in both samples with and without cocaine, the maximum signal enhancement fold of 0.28 for cocaine after 30-min of Exo III digestion was observed at $300 \mu \mathrm{M}$ (Figure $63 \mathrm{D}$ and F). At low concentrations of DTT such as 100 and $200 \mu \mathrm{M}$, the surface density of SF-CBSA-5345 on the AuNPs is too high therefore limiting the assembly efficiency of the SF-CBSA-5345 with the LF-CBSA-5345 to form the cocaine-CBSA complex, and preventing the specific enzyme digestion (Figure 63B and $63 \mathrm{C}$ ) resulting in a poor signal enhancement fold of 0.08 and 0.25 , respectively (Figure 63F). At very high DTT concentration such as $400 \mu \mathrm{M}$ resulted in very few SFCBSA-5345 fragments remained on the surface, indicated by an aggregated state even before addition of Exo III (Fig. 63E), therefore unfavorable for sensitive colorimetric detection. Thus, we chose an optimum DTT concentration of $300 \mu \mathrm{M}$, which has enough SF-CBSA-5345 fragments on the surface of the AuNP to remain in non-aggregated state and facilitate high assembly efficiency of cocaine-CBSA complex and faster Exonuclease kinetics in the presence of cocaine compared to its absence. 

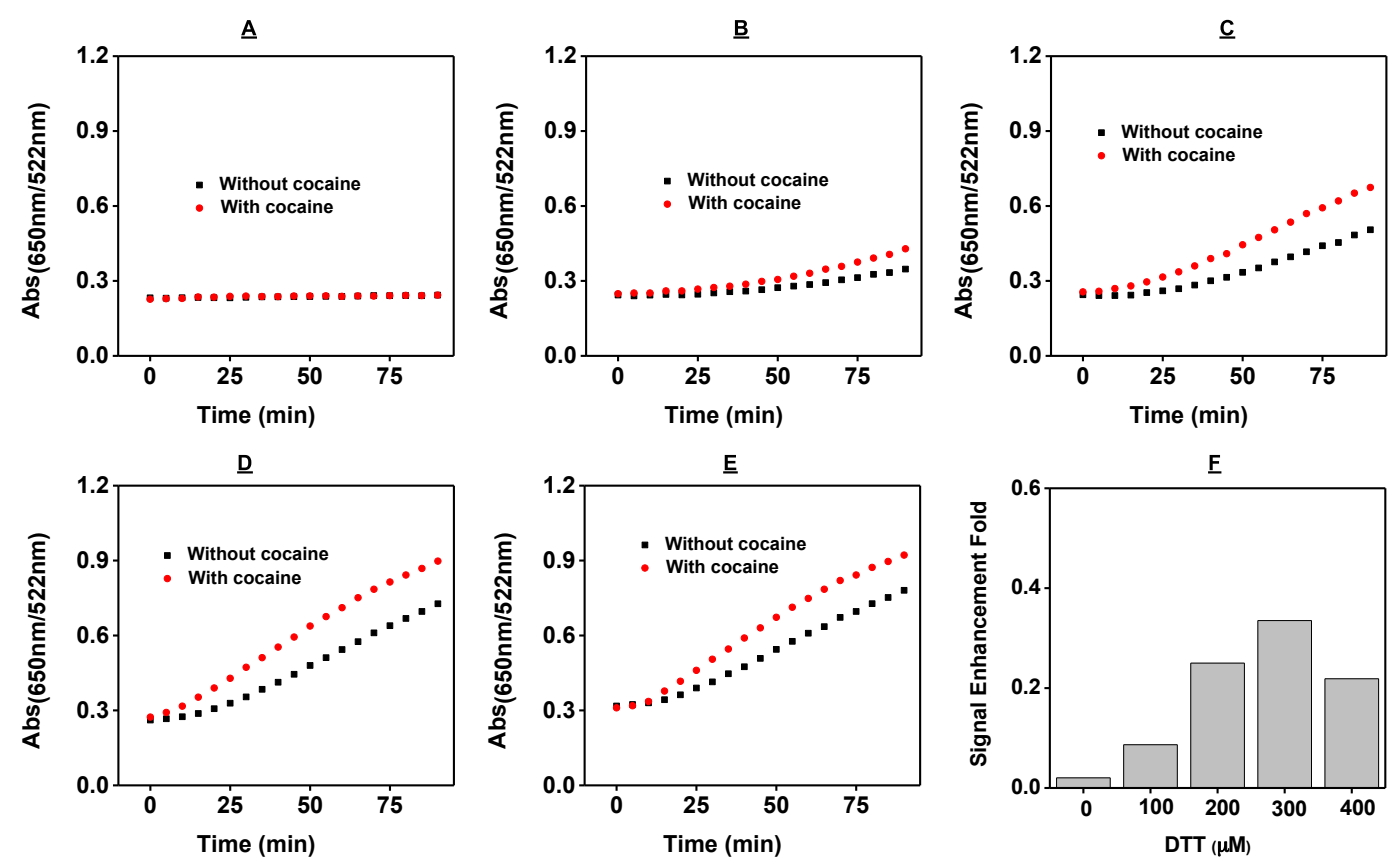

Figure 63. Effect of DTT concentration on the surface coverage of SF-CBSA-5345 resulting in change of Exo III kinetics for cocaine detection. The SF-CBSA-5345modified AuNPs were first treated with different concentration of DTT $(0-400 \mu \mathrm{M})$; (A) $0 \mu \mathrm{M}$; (B) $100 \mu \mathrm{M}$; (C) $200 \mu \mathrm{M}$; (D) $300 \mu \mathrm{M}$; and (E) $400 \mu \mathrm{M}$ to obtain different surface coverages. (F) Signal enhancement fold between cocaine and DI calculated with $\mathrm{A}_{650} / \mathrm{A}_{522}$ after 30 -min Exo III digestion.

Using the optimized surface coverage of SF-CBSA-5345, we further tested the LFCBSA-5345 concentration effect on target-CBSA assembly and Exo digestion by varying the concentrations from 1 to $0 \mu \mathrm{M}$ (Figure $64 \mathrm{~A}, \mathrm{~B}, \mathrm{C}, \mathrm{D}$ and E). Faster enzyme digestion was observed in the samples with and without cocaine, when the concentration of LFCBSA-5345 is $1 \mu \mathrm{M}$ (Figure 64A) without any specificity, whereas slight improvement in specificity was observed at LF-CBSA-5345 concentrations of 100 and $50 \mathrm{nM}$ with optimum signal enhancement fold of 0.2 for both (Figure 64F). Clearly, there is only a slight improvement in the stability and specificity of the cocaine-CBSA complex when we increased the number of bases after the AP site to 4 at the 3' end of SF-CBSA-5345. 
Therefore, we tested the newly designed CBSA-5335 to further improve the stability of the cocaine-CBSA complex and enhance the specificity of enzyme digestion.
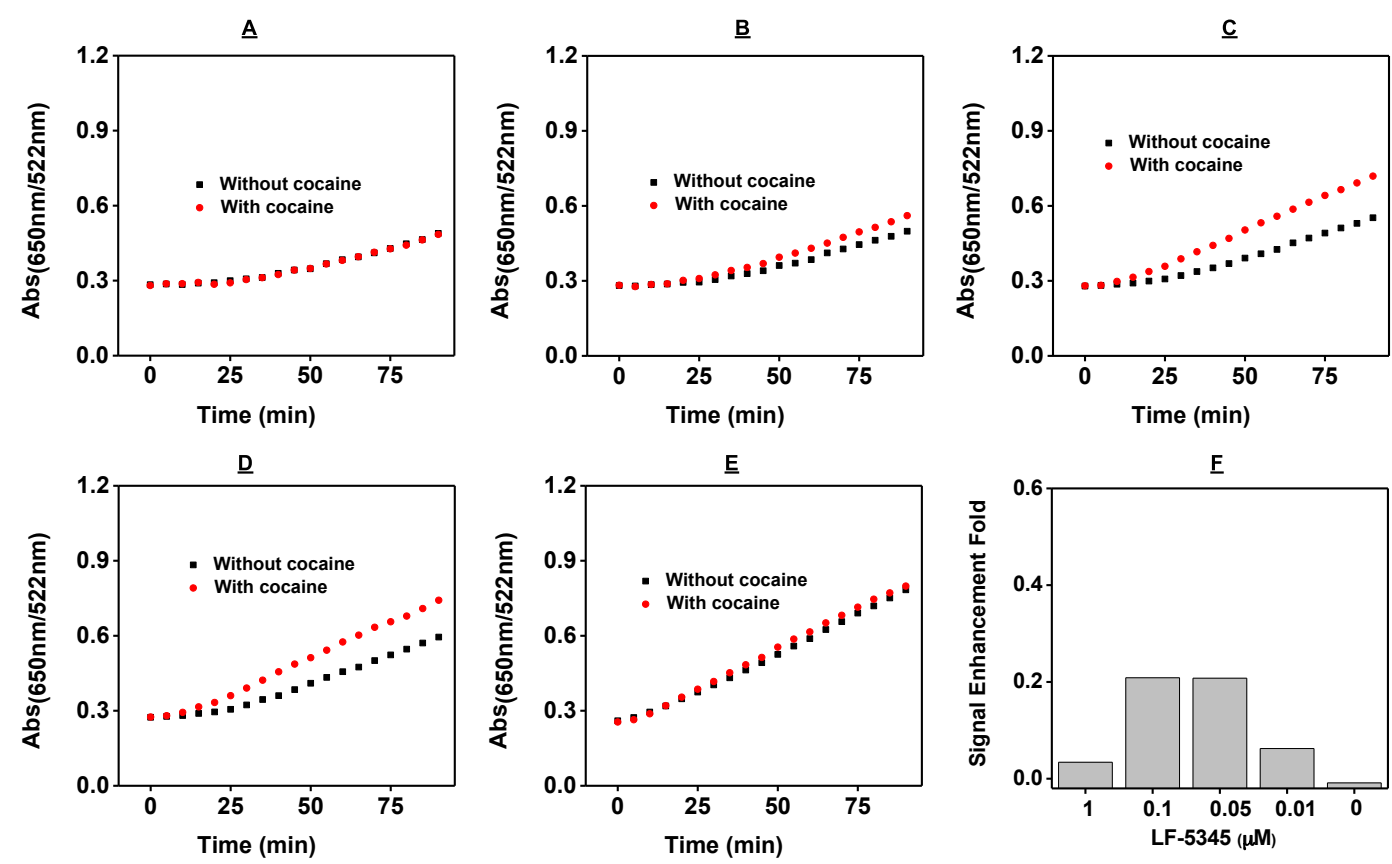

Figure 64. Effect of LF-CBSA-5345 concentration on the efficiency of target-aptamer assembly and Exo III kinetics for cocaine detection. The SF-CBSA-5345-modified AuNPs were first treated with optimized DTT concentration of $300 \mu \mathrm{M}$ to obtain the surface coverage of 26 DNA strands per AuNP, to which variable concentrations of LFCBSA-5345 were added ranging from $1 \mu \mathrm{M}$ to $0 \mathrm{nM}$. (A) $1 \mu \mathrm{M}$; (B) $0.1 \mu \mathrm{M}$; (C) 0.05 $\mu \mathrm{M}$; (D) $0.01 \mu \mathrm{M}$; and (E) $0 \mathrm{nM}$. (F) Signal enhancement fold between cocaine and DI calculated with $\mathrm{A}_{650} / \mathrm{A}_{522}$ after 30 -min Exo III digestion.

DTT incubation assay was performed on the SF-CBSA-5335-modified AuNPs, the same way as described above to optimize the surface density of SF-CBSA-5335 to achieve maximum signal enhancement fold with faster reaction rate. An optimum surface coverage of the SF-CBSA-5335 was obtained with the DTT concentration of $100 \mu \mathrm{M}$ (Figure 65C) with a maximum signal enhancement fold of 0.5 (Figure 65E). This is quite a significant improvement in the specificity and the speed of the CBSA-based, Exo digestion compared to the cocaine-aptamer complex formed with the CBSA-5345. Therefore, we decided to keep the AP site equidistant from the two binding domains in an 
attempt to improve stability and duplex formation so that Exo III can bind to the AP site of the folded cocaine-CBSA complex in the presence of cocaine. Optimizing the surface coverage was however essential to get maximum signal enhancement fold.
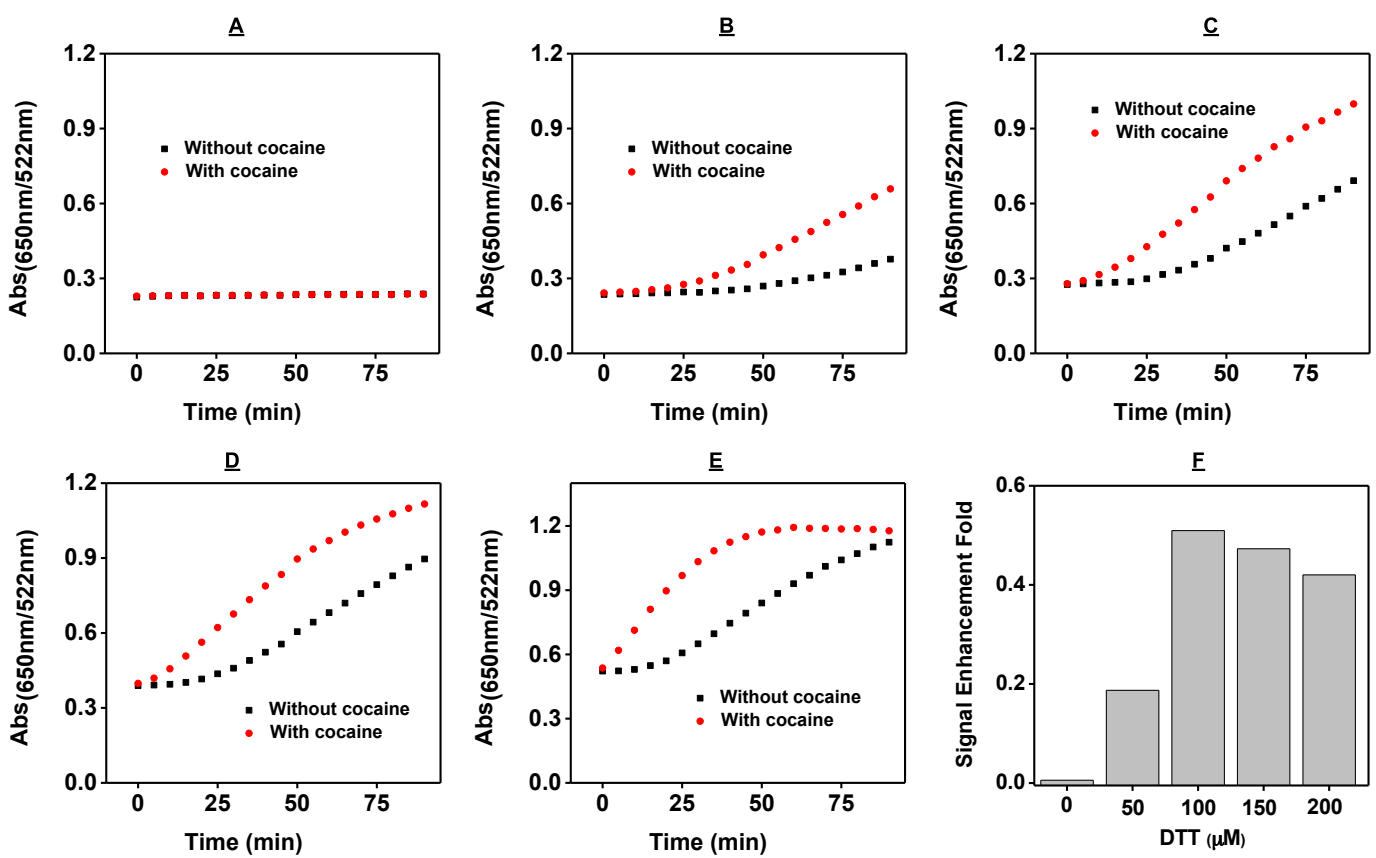

Figure 65. Effect of DTT concentration on the surface coverage of SF-CBSA-5335 resulting in change of Exo III kinetics for cocaine detection. The SF-CBSA-5335modified AuNPs were first treated with different concentration of DTT $(0-150 \mu \mathrm{M})$; (A) $0 \mu \mathrm{M}$; (B) $50 \mu \mathrm{M}$; (C) $100 \mu \mathrm{M}$; and (D) $150 \mu \mathrm{M}$ to obtain different surface coverages. (E) Signal enhancement fold between cocaine and DI calculated with $\mathrm{A}_{650} / \mathrm{A}_{522}$ after 30-min Exo III digestion. 

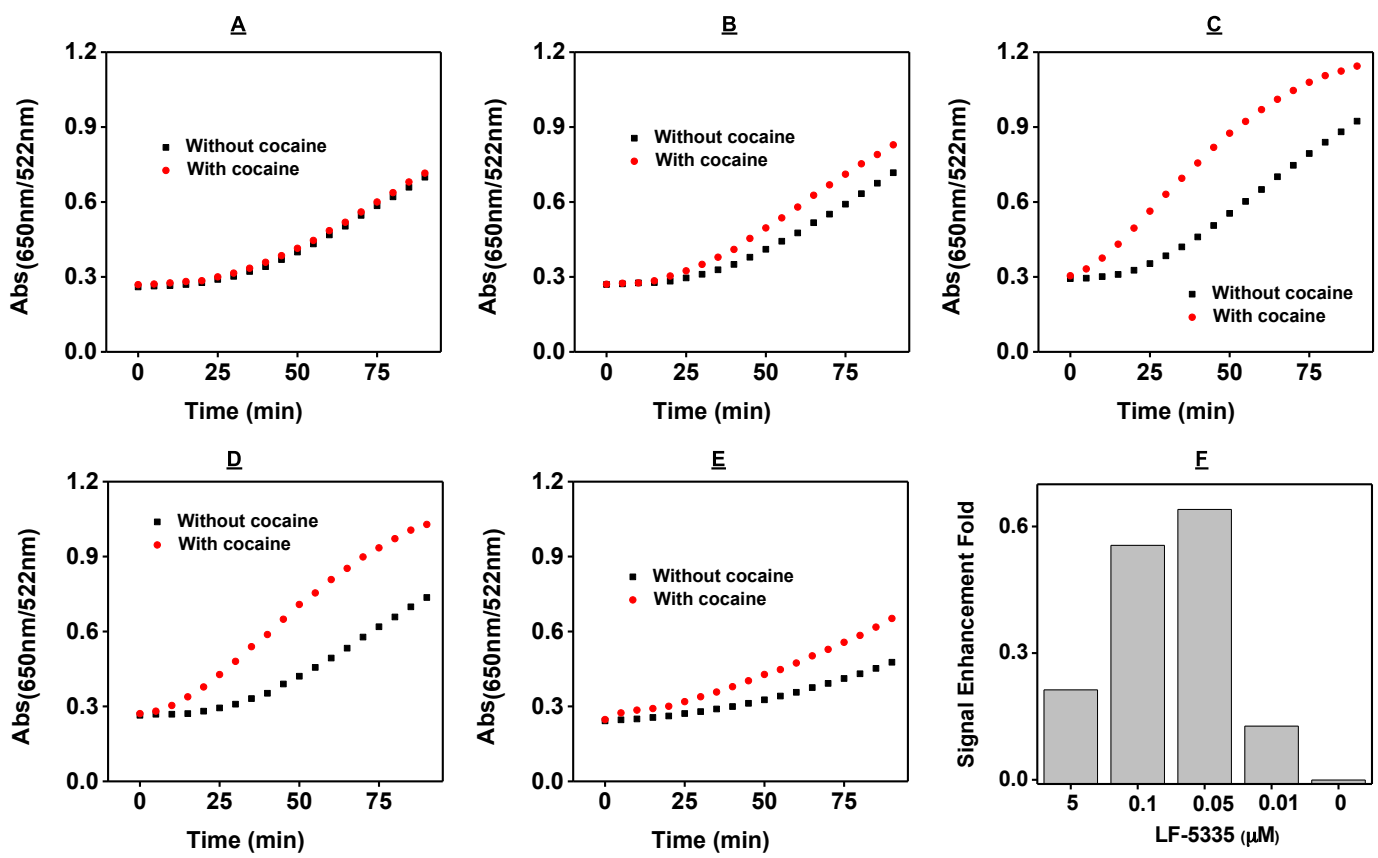

Figure 66. Effect of LF-CBSA-5335 concentration on the efficiency of target-aptamer assembly and Exo III kinetics for cocaine detection. The SF-CBSA-5335-modified AuNPs were first treated with optimized DTT concentration of $100 \mu \mathrm{M}$ to obtain the surface coverage of 30 DNA strands per AuNP, to which variable concentrations of LFCBSA-5335 were added ranging from $5 \mu \mathrm{M}$ to $0 \mathrm{nM}$. (A) $5 \mu \mathrm{M}$; (B) $0.1 \mu \mathrm{M}$; (C) 0.05 $\mu \mathrm{M}$; (D) $0.01 \mu \mathrm{M}$; and (E) $0 \mathrm{nM}$. (F) Signal enhancement fold between cocaine and DI calculated with $\mathrm{A}_{650} / \mathrm{A}_{522}$ after 30-min Exo III digestion.

The concentration of LF-CBSA-5335 was further optimized to improve the assay's specificity. Using the optimized surface coverage of SF-CBSA-5335 treated with $100 \mu \mathrm{M}$ DTT, we further tested the LF-CBSA-5335 concentration effect on cocaine-CBSA assembly and Exo digestion by varying the concentrations from 5 to $0 \mu \mathrm{M}$ (Figure $66 \mathrm{~A}$, $\mathrm{B}, \mathrm{C}, \mathrm{D}$ and E). When the LF-CBSA-5335 concentration was decreased to $50 \mathrm{nM}$, we observed a clear distinct purple color change occurring at $45 \mathrm{~min}$ in the presence of cocaine and achieved the highest signal enhancement fold of 0.6 (Figure 66C, 66F). However, it took at least 45 min to observe a distinct deep purple color change even with the optimized SF-CBSA-5335 surface coverage and LF-CBSA-5335 concentration (Figure 66C), whereas the control without cocaine still remained red. Therefore we 
engineered another CBSA based on CBSA-5335 to further increase specific Exo digestion speed and reduce the formation of non-specific target-CBSA complex.

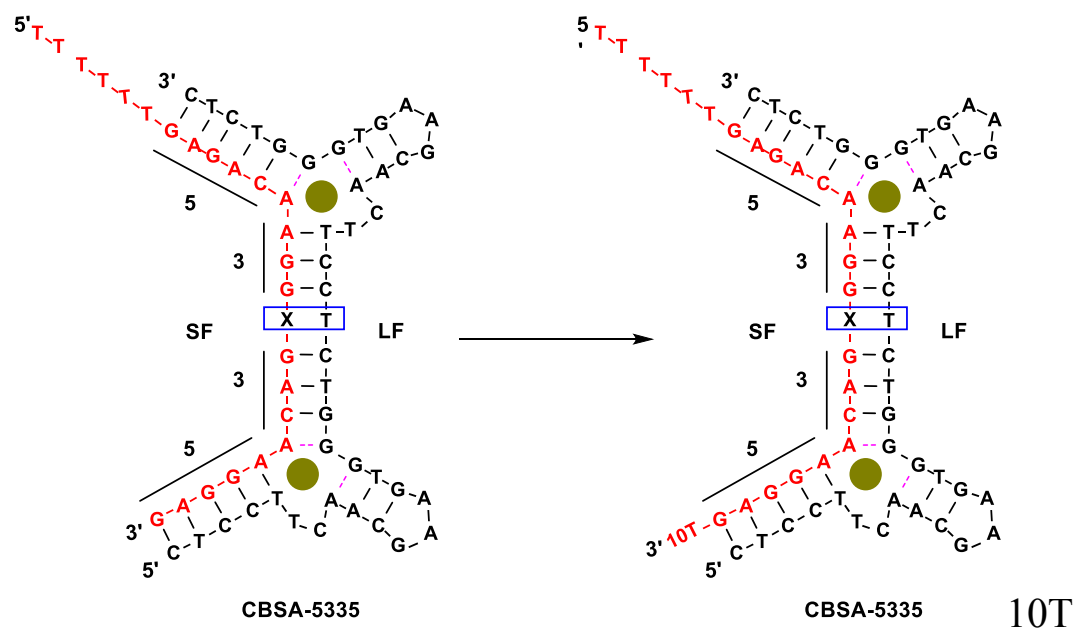

Figure 67. Utilizing CBSA-5335 to engineer CBSA-5335-10T. CBSA Structure of folded split cocaine aptamer with dual binding domain assembled in the presence of cocaine comprising of short fragment and a long fragment.

Building on the sequence design of SF-CBSA-5335 for improved specificity of the stable cocaine-CBSA complex, another modification was made to the 3' end of SF-CBSA-5335 by adding $10 \mathrm{~T}$ linker therefore referred to as SF-CBSA-5335-10T (Figure 67). We also assume that the addition of 10T linker in SF-CBSA-5335-10T may favor the formation of cocaine-CBSA complex and Exonuclease digestion at a relatively faster rate. These thymine bases at the 3' end of the SF-5335-10T may reduce the non-specific enzyme digestion in the absence of cocaine, therefore improving the signal enhancement fold. Note that the LF-CBSA-5335-10T remains the same as the LF-CBSA-5335.

We first changed the DTT concentrations from 0 to $500 \mu \mathrm{M}$, however keeping the long fragment concentration to $50 \mathrm{nM}$ as optimized earlier for S-CBSA-5335, to optimize the surface coverage using DTT incubation assay on the SF-CBSA-5335-10T-modified AuNPs, the same way as described above. An optimum surface coverage of the SF- 
CBSA-5335-10T was obtained with the DTT concentration of $400 \mu \mathrm{M}$ (Figure 68D) with a maximum signal enhancement fold of 0.6 (Figure 68F). This is a slight improvement in the specificity and the speed of the Exo digestion compared to the cocaine-CBSA complex formed with the CBSA-5335, but the samples without cocaine are digested at a slower rate (Figure $68 \mathrm{~A}, \mathrm{~B}, \mathrm{C}, \mathrm{D}$, and E). The long fragment remains same as the one we used for SF-CBSA-5335. An optimum signal enhancement fold was observed for 400 $\mu \mathrm{M}$ DTT with the color change occurring at around $40 \mathrm{~min}$ (Figure 68F). Using the optimized DTT, the concentration of long fragment was optimized again. As expected, 50 nM LF-CBSA-5335 gave the highest signal enhancement fold of 0.65 (Figure 69F) with the color change reaction in the presence of cocaine after 40 min (Figure 69C).
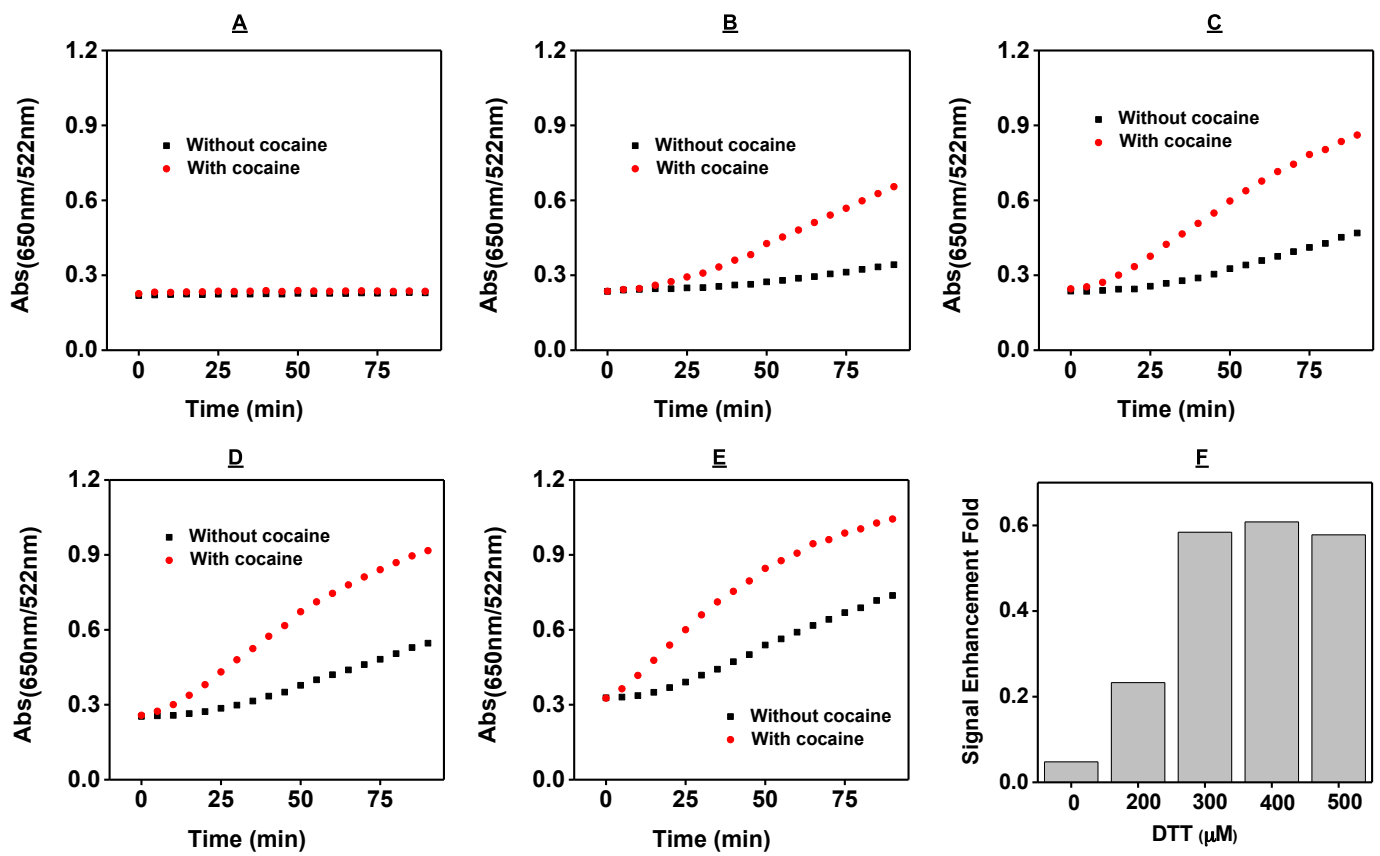

Figure 68. Effect of DTT concentration on the surface coverage of SF-CBSA-5335-10T resulting in change of Exo III kinetics for cocaine detection. The SF-CBSA-5335-10Tmodified AuNPs were first treated with different concentration of DTT $(0-500 \mu \mathrm{M})$; (A) $0 \mu \mathrm{M}$; (B) $200 \mu \mathrm{M}$; (C) $300 \mu \mathrm{M}$; (D) $400 \mu \mathrm{M}$; and (D) $500 \mu \mathrm{M}$ to obtain different surface coverages. (E) Signal enhancement fold between cocaine and DI calculated with $\mathrm{A}_{650} / \mathrm{A}_{522}$ after 30-min Exo III digestion. 

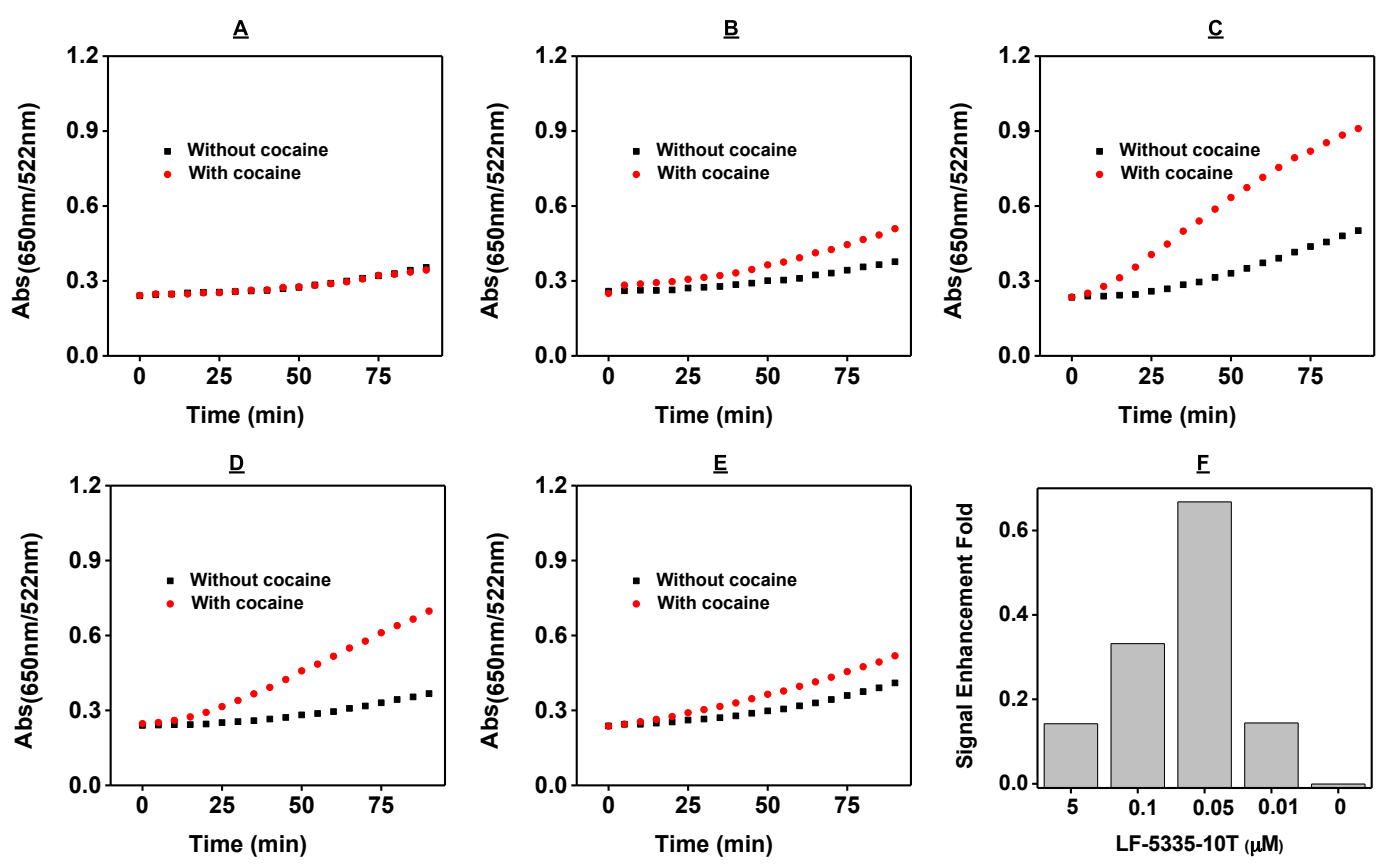

Figure 69. Effect of LF-CBSA-5335 concentration on the efficiency of target-aptamer assembly and Exo III kinetics for cocaine detection. The SF-CBSA-5335-10T-modified AuNPs were first treated with optimized DTT concentration of $400 \mu \mathrm{M}$ to obtain the surface coverage of 30 DNA strands per AuNP, to which variable concentrations of LFCBSA-5335 were added ranging from $5 \mu \mathrm{M}$ to $0 \mathrm{nM}$. (A) $0 \mu \mathrm{M}$; (B) $0.01 \mu \mathrm{M}$; (C) 0.05 $\mu \mathrm{M}$; (D) $0.1 \mu \mathrm{M}$; and (E) $5 \mu \mathrm{M}$. (F) Signal enhancement fold between cocaine and DI calculated with $\mathrm{A}_{650} / \mathrm{A}_{522}$ after 30-min Exo III digestion.

\subsection{Conclusions}

To conclude this chapter, we first compared different aptamer sequence combinations with single binding domain by optimizing the surface coverage of the short aptamer fragment on the AuNP using the DTT displacement assay. We further characterized the accurate surface coverage at each of those DTT concentrations using the OliGreen assay. The effect of long aptamer fragment concentration was studied and optimized for improved specificity. We have also studied the effect of incorporation of the second binding domain with variable chain lengths on either side of the AP site. Based on our results, we finally shortlisted the sequence combination with maximum signal enhancement fold to modify and synthesize the sequence with two AP sites, which is 
instrumental in faster, and specific reaction to observe a distinctive color change in the presence of cocaine. In the future, we will focus on application of the system in the real world samples such as saliva to evaluate its sensing performance. 


\section{CHAPTER 6: Summary and Future Work}

\subsection{Summary}

This dissertation describes the combination of CNTs and AuNPs with simple fabrication techniques like vacuum filtration to create sensor platforms for multi-analyte detection in complex samples, as well as the generation of an instrument-free platform that combines AuNPs and DNA aptamers for the colorimetric detection of cocaine. The focus of this study was to develop suitable on-site small-molecule detection strategies using low-cost nanomaterials and energy-efficient methods for sensitive analyte detection based on the following strategies: i) using M-SWCNTs and unmodified AuNPs for the construction of highly-conductive porous gold films on MCE filter paper using vacuum filtration, which are suitable for electrochemical detection of multiple analytes (e.g. DA and 5-HT); ii) using vacuum filtration to fabricate a thin P-SWCNT film on MCE paper to detect $\mathrm{NADH}$ in complex biosamples; and iii) engineering aptamer sequences as a means to optimize the speed and specificity of an enzymatic reaction for amplified colorimetric cocaine detection using AuNPs.

We report here a simple and novel strategy for preparing disposable, paper-based, porous AuNP/M-SWCNT hybrid thin gold films with high conductivity, rapid electron transfer rates and excellent electrocatalytic properties that take $\sim 20$ minutes to prepare. The entire process requires only two steps of ambient vacuum filtration with two aqueous solutions containing M-SWCNTs and AuNPs, which respectively form a porous underlayer comprising a network of interconnected ligaments and pores and an upper layer of citrate-coated AuNPs that assemble into a conductive and porous thin gold film. These films are prepared at room temperature in a highly reproducible fashion without the need 
for sophisticated instruments or a clean-room environment. Our vacuum filtration fabricated gold films can distinguish the presence of DA and 5-HT either individually or simultaneously, with a resolution that greatly exceeds that of commercially available flat gold slides or porous gold films prepared by more labor-intensive electrodeposition techniques. We have also further expanded our vacuum filtration fabrication technique to generate porous P-SWCNT film electrodes for the electrochemical detection of both naturally available and enzymatically-generated NADH in complex matrices such as saliva and cellular extracts. Quantification of NADH in such complex matrices can provide valuable information for disease diagnosis and drug detection, and our assay yielded results that are comparable with commercial colorimetric assays. By using filter paper as a substrate to support these nanomaterials, we were in both instances able to add to the virtues of our composite films by making the resulting devices flexible, cheap, and biodegradable, while providing a porous matrix that traps the nanotubes during the vacuum filtration to simplify the fabrication process. Finally, we have developed an instrument-free colorimetric detection strategy for practical POC applications, in which we used AuNPs modified with DNA aptamers to sensitively detect cocaine. Importantly, by comparing different sequence combinations with single and dual binding domains and optimizing the surface coverage of aptamer fragments on the AuNP, we were able to further enhance the performance of our detection assay.

\subsection{Future work}

Looking at existing paper-based sensors, it is evident that there has been extensive development over the last few years that has led to "smarter" paper-based sensors. Nanomaterials such as carbon nanotubes or graphene can potentially be used as a 
conductive underlay for the deposition of a variety of different nanoparticles, including gold, silver, copper, platinum or palladium, to form various pure or alternating layers of metal films. Such hybrid metal films could be used in electrochemical catalysis or electro-optical devices, as well as in reflective, conductive or energy-collecting metallic coatings. We believe that our technique delivers a simple, adaptable and general means for the rapid fabrication of such diverse metal films onto membranes under ambient conditions, and that this process could likewise serve as a prelude to the transfer of such films to other metal or flexible substrates for diverse applications. Although there is huge potential in paper as a substrate for on-site detection devices, current strategies for fabrication and analysis still lack the performance and robustness of standard analytical techniques. Further research related to fabrication techniques and the incorporation of novel functional materials onto the surface is needed to overcome the challenges involved with developing a more stable platform capable of measuring multiple analytes in complex samples with high sensitivity. And although colorimetric techniques offer a suitable strategy, as described in this thesis, for achieving EATR-amplified cocaine detection with high specificity, working with real-world samples such as saliva is likely to pose additional challenges that will need to be addressed in future research. 


\section{References}

1. Barizuddin, S.; Bok, S.; Gangopadhyay, S. Plasmonic Sensors for Disease Detection - A Review. J. Nanomed. Nanotechnol. 2016, 7, 10000373.

2. Yáñez-Sedeño P.; Agüí, L.; Villalonga, R.; Pingarrón, J. M. Biosensors in Forensic Analysis. A Review. Anal. Chim. Acta 2014, 823, 1-19.

3. Mozaz, S. R.; López de-Alda, M. J.; Marco, M. P.; Barceló, D. Biosensors for Environmental Monitoring: A Global Perspective. Talanta 2005, 65, 291-297.

4. Yu, D.; Blankert, B.; Viré, J. C.; Kauffmann, J. M. Biosensors in Drug Discovery and Drug Analysis. Anal. Lett. 2005, 38, 1687-1701.

5. Narsaiah, K.; Jha, S. N.; Bhardwaj, R.; Sharma, R.; Kumar, R. Optical Biosensors for Food Quality and Safety Assurance-A Review. J. Food Sci. Technol. 2012, $49,383-406$.

6. Clark Jr., L. C.; Lyons, C. Electrode Systems for Continuous Monitoring in Cardiovascular Surgery. Ann. N. Y. Acad. Sci. 1962, 102, 29-45.

7. Zhang, M.; Li, J. Carbon Nanotube in Different Shapes. Mater. Today 2009, 12, 12-18.

8. MacKenzie, K. J.; Dunens, O. M.; See, C. H.; Harris, A. T. Large-Scale Carbon Nanotube Synthesis. Recent Pat Nanotechnol. 2008, 2, 25-40.

9. White, C. T.; Mintmire, J. W. Fundamental Properties of Single-Wall Carbon Nanotubes. J. Phys. Chem. B, 2005, 109, 52-65.

10. Cantoro, M.; Hofmann, S.; Pisana, S.; Scardaci, V.; Parvez, A.; Ducati, C.; Ferrari, A. C.; Blackburn, A. M.; Wang, K. Y.; Robertson, J. Catalytic Chemical Vapor Deposition of Single-Wall Carbon Nanotubes at Low Temperatures. Nano Lett. 2006, 6, 1107-1112.

11. Hu, C.; Hu, S. Carbon Nanotube-Based Electrochemical Sensors: Principles and Applications in Biomedical Systems. J. Sens. 2009, 187615.

12. Saifuddin, N.; Raziah, A. Z.; Junizah, A. R. Carbon Nanotubes: A Review on Structure and Their Interaction with Proteins. J. Chem. 2013, 676815.

13. Lohse, S. E.; Murphy, C. J. Applications of Colloidal Inorganic Nanoparticles: From Medicine to Energy. J. Am. Chem. Soc. 2012, 134, 15607-15620.

14. Saha, K.; Agasti, S. S.; Kim, C.; Li, X.; Rotello, V. M. Gold Nanoparticles in Chemical and Biological Sensing. Chem. Rev. 2012, 112, 2739-2779. 
15. Thompson, D. T. Using Gold Nanoparticles for Catalysis. Nano Today 2007, 2, 40-43.

16. Teranishi, T. Fabrication and Electronic Properties of Gold Nanoparticle Superlattices. C. R. Chim. 2003, 6, 979-987.

17. Popovtzer, R.; Agrawal, A.; Kotov, N. A.; Popovtzer, A.; Balter, J.; Carey, T. E.; Kopelman, R. Targeted Gold Nanoparticles enable Molecular CT Imaging of Cancer. Nano Lett. 2008, 8, 4593-4596.

18. Dykman, L. A.; Khlebstov, N. G. Gold Nanoparticles in Biology and Medicine: Recent Advances and Prospects. Acta Naturae 2011, 3, 34-55.

19. Gadogbe, M.; Ansar, S. M.; He, G.; Collier, W. E.; Rodriguez, J.; Liu, D.; Chu, I. W.; Zhang, D. Determination of Colloidal Gold Nanoparticle Surface Areas, Concentrations, and Sizes through Quantitative Ligand Adsorption. Anal. Bioanal. Chem. 2013, 405, 413-422.

20. Homberger, M.; Simon, U. On the Application Potential of Gold Nanoparticles in Nanoelectronics and Biomedicine. Philos. Trans. R. Soc. A 2010, 368, 1405-1453.

21. Sajanlal, P. R.; Sreeprasad, T. S.; Samal, A. K.; Pradeep, T. Anisotropic Nanomaterials: Structure, Growth, Assembly, and Functions. Nano Rev. 2011, 2, 5883 .

22. Huang, X.; El-Sayed, M. A. Gold nanoparticles: Optical Properties and Implementations in Cancer Diagnosis and Photothermal Therapy. Adv. Res. 2010, $1,13-28$.

23. Turkevich, J.; Stevenson, P. L.; Hillier, J. A study of the nucleation and growth process in the synthesis of colloidal gold. Discuss. Faraday Soc. 1951, 11, 55-75.

24. Ji, X.; Song, X.; Li, J.; Bai, Y.; Yang, W.; Peng, X. Size Control of Gold Nanocrystals in Citrate Reduction: The Third Role of Citrate. J. Am. Chem. Soc. 2007, 129, 13939-13948.

25. Li, C.; Li, D.; Wan, G.; Xu, J.; Hou, W. Facile Synthesis of Concentrated Gold Nanoparticles with Low Size-Distribution in Water: Temperature and $\mathrm{pH}$ Controls. Nanoscale Res. Lett. 2011, 6, 440.

26. Toma, H. E.; Zamarion, V. M.; Toma, S. H.; Araki, A. The Coordination Chemistry at Gold Nanoparticles. J. Braz. Chem. Soc. 2010, 21, 1158-1176.

27. Tyagi, H.; Kushwaha, A.; Kumar, A.; Aslam, M. A Facile pH Controlled CitrateBased Reduction Method for Gold Nanoparticle Synthesis at Room Temperature. Nanoscale Res. Lett. 2016, 11, 362. 
28. Lu, X.; Tuan, H. Y.; Korgel, B. A.; Xia, Y. Facile Synthesis of Gold Nanoparticles With Narrow Size Distribution by Using $\mathrm{AuCl}$ or $\mathrm{AuBr}$ as the Precursor. Chemistry 2008, 14, 1584-1591.

29. A Comparison of TEM and DLS Methods to Characterize Size Distribution of Ceramic Nanoparticles. J. Phys. Conference Series 2016, 733, 012039.

30. Shaw, J. L. V. Practical Challenges Related to Point of Care Testing. Practical Laboratory Medicine 2016, 4, 22-29.

31. Ko, W. Y.; Lin, K. J. Highly Conductive, Transparent Flexible Films Based on Metal Nanoparticle-Carbon Nanotube Composites. J. Nanomater. 2013, 505292.

32. Satishkumar, B. C.; Vogl, E. M.; Govindaraj, A.; Rao, C. N. R. The Decoration of Carbon Nanotubes by Metal Nanoparticles. J. Phys. D: Appl. Phys. 1996, 29, 3173.

33. Banerjee, S.; Wong, S. S. Functionalization of Carbon Nanotubes with a MetalContaining Molecular Complex. Nano Lett. 2002, 2, 49-53.

34. Jiang, Z.; Jiang, Z. J. Carbon Nanotubes Supported Metal Nanoparticles for the Applications in Proton Exchange Membrane Fuel Cells. Carbon Nanotubes Growth and Applications, InTech Inc. 2011, 567-604.

35. Liu, X.; Marangon, I.; Melinte, G.; Wilhelm, C.; Moyon, C. M.; Pichon, B. P.; Ersen, O.; Aubertin, K.; Baaziz, W.; Huu, C. P.; Colin, S. B.; Bianco, A.; Gazeau, F.; Begin, D. Design of Covalently Functionalized Carbon Nanotubes Filled with Metal Oxide Nanoparticles for Imaging, Therapy, and Magnetic Manipulation. ACS Nano 2014, 8, 11290-11304.

36. Xia, Y.; Deng, X.; Yan, S.; Gao, C.; Ma, C.; Jin, L. Nanoporous Gold Film: Fabrication and Role as a Catalytic Reactor. Dalton Trans. 2015, 44, 1192911934.

37. Zhang, R.; Olin, H. Porous Gold Films - A Short Review on Recent Progress. Materials 2014, 7, 3834-3854.

38. Gonçales, V. R.; Massafera, M. P.; Benedetti, T. M.; Moore, D. G.; De Torresi, S. I. C.; Torresi, R. M. Nanostructured Thin Films Obtained by Electrodeposition over a Colloidal Crystal Template: Applications in Electrochemical Devices. $J$. Braz. Chem. Soc. 2009, 20, 663-673.

39. Wang, D.; Luo, H.; Kou, R.; Gil, M. P.; Xiao, S.; Golub, V. O.; Yang, Z.; Brinker, C. J.; Lu, Y. A General Route to Macroscopic Hierarchical 3D Nanowire Networks. Angew. Chem., Int. Ed. 2004, 43, 6169-6173. 
40. Ismail, I. M. I. Synthesis of Nanostructured Films from Template Electrodeposition Technique. J. Saudi Chem. Soc. 2010, 14, 351-356.

41. Szamocki, R.; Reculusa, S.; Ravaine, S.; Bartlett, P. N.; Kuhn, A.; Hempelmann, R. Tailored Mesostructuring and Biofunctionalization of Gold for Increased Electroactivity. Angew. Chem., Int. Ed. 2006, 45, 1317-1321.

42. Velev, O. D.; Tessier, P. M.; Lenhoff, A. M.; Kaler, E. W. Materials: A Class of Porous Metallic Nanostructures. Nature 1999, 401, 548.

43. Kim, M.; Ha, W. J.; Anh, J. W.; Kim, H. S.; Park, S. W.; Lee, D. Fabrication of Nanoporous Gold Thin Films on Silicon Substrate by Multilayer Deposition of Au and Ag. J. Alloys Compd. 2009, 484, 28-32.

44. Malinský, P.; Slepička, P.; Hnatowicz, V.; Švorčík, V. Early Stages of Growth of Gold Layers Sputter Deposited on Glass and Silicon Substrates. Nanoscale Res. Lett. 2012, 7, 241.

45. Xu, X.; Jia, J.; Yang, X.; Dong, S. A Templateless, Surfactantless, Simple Electrochemical Route to a Dendritic Gold Nanostructure and Its Application to Oxygen Reduction. Langmuir 2010, 26, 7627-7631.

46. Cherevko, S.; Chung, C. H. Direct Electrodeposition of Nanoporous Gold with Controlled Multimodal Pore Size Distribution. Electrochem. Commun. 2011, 13, 16-19.

47. Najdovski, I.; Selvakannan, P. R.; Bhargava, S. K.; O’Mullane, A. P. Formation of Nanostructured Porous $\mathrm{Cu}-\mathrm{Au}$ Surfaces: The Influence of Cationic Sites on (Electro)-Catalysis. Nanoscale 2012, 4, 6298-6306.

48. Schönenberger, C.; van der Zande, B. M. I.; Fokkink, L. G. J.; Henny, M.; Schmid, C.; Krüger, M.; Bachtold, A.; Huber, R.; Birk, H.; Staufer, U. Template Synthesis of Nanowires in Porous Polycarbonate Membranes: Electrochemistry and Morphology. J. Phys. Chem. B 1997, 101, 5497-5505.

49. Wijnhoven, J. E. G. J.; Zevenhuizen, S. J. M.; Hendriks, M. A.; Vanmaekelbergh, D.; Kelly, J. J.; Vos, W. L. Electrochemical Assembly of Ordered Macropores in Gold. Adv. Mater. 2000, 12, 888-890.

50. Bartlett, P. N.; Baumberg, J. J.; Birkin, P. R.; Ghanem, M. A.; Netti, M. C. Highly Ordered Macroporous Gold and Platinum Films Formed by Electrochemical Deposition through Templates Assembled from Submicron Diameter Monodisperse Polystyrene Spheres. Chem. Mater. 2002, 14, 2199-2208. 
51. Ye, W.; Yan, J.; Ye, Q.; Zhou, F. Template-Free and Direct Electrochemical Deposition of Hierarchical Dendritic Gold Microstructures: Growth and Their Multiple Applications. J. Phys. Chem. C 2010, 114, 15617-15624.

52. Li, Y.; Song, Y. Y.; Yang, C.; Xia, X. Hydrogen Bubble Dynamic Template Synthesis of Porous Gold for Nonenzymatic Electrochemical Detection of Glucose. Electrochem. Commun. 2007, 9, 981-988.

53. Ding, Y.; Kim, Y. J.; Erlebacher, J. Nanoporous Gold Leaf: "Ancient Technology"/Advanced Material. Adv. Mater. 2004, 16,1897-1900.

54. El Mel, A. A.; Boukli-Hacene, F.; Molina-Luna, L.; Bouts, N.; Chauvin, A.; Thiry, D.; Gautron, E.; Gautier, N.; Tessier, P. Y. Unusual Dealloying Effect in Gold/Copper Alloy Thin Films: The Role of Defects and Column Boundaries in the Formation of Nanoporous Gold. ACS Appl. Mater. Interfaces 2015, 7, $2310-2321$.

55. Erlebacher, J.; Aziz, M. J.; Karma, A.; Dimitrov, N.; Sieradzki, K. Evolution of Nanoporosity in Dealloying. Nature 2001, 410, 450-453.

56. Ge, S.; Ge, L.; Yan, M.; Song, X.; Yu, J.; Huang, J. A Disposable Paper-Based Electrochemical Sensor With an Addressable Electrode Array for Cancer Screening. Chem. Commun. 2012, 48, 9397-9399.

57. Liana, D. D.; Raguse, B.; Gooding, J. J.; Chow, E. Recent Advances in PaperBased Sensors. Sensors 2012, 12, 11505-11526.

58. Mostafalu, P.; Akbari, M.; Alberti, K. A.; Xu, Q.; Khademhosseini, A.; Sonkusale, S. R. A Toolkit of Thread-Based Microfluidics, Sensors, and Electronics for 3D Tissue Embedding for Medical Diagnostics. Microsyst. Nanoeng. 2016, 2, 16039.

59. Silveira, C. M.; Monteiro, T.; Almeida, M. G. Biosensing with Paper-Based Miniaturized Printed Electrodes-A Modern Trend. Biosensors 2016, 6, 51.

60. Liu, Z.; Wang, X.; Li, M.; Wu, W. Tunnelling Conductive Hybrid Films of Gold Nanoparticles and Cellulose and their Applications as Electrochemical Electrodes. Nanotechnology 2015, 26, 465708.

61. Martinez, A. W.; Phillips, S. T.; Whitesides, G. M. Diagnostics for the Developing World: Microfluidic Paper-Based Analytical Devices. Anal. Chem. 2010, 82, 3-10.

62. Tobjörk, D.; Aarnio, H.; Pulkkinen, P.; Bollström, R.; Määttänen, A.; Ihalainen, P.; Mäkelä, T.; Peltonen, J.; Toivakka, M.; Tenhu, H.; Österbacka, R. IR- 
Sintering of Ink-Jet Printed Metal-Nanoparticles on Paper. Thin Solid Films 2012, 520, 2949-2955.

63. Määttänen, A.; Ihalainen, P.; Pulkkinen, P.; Wang, S.; Tenhu, H.; Peltonen, J. Inkjet-Printed Gold Electrodes on Paper: Characterization and Functionalization. ACS Appl. Mater. Interfaces 2012, 4, 955-964.

64. Määttänen, A.; Vanamo, U.; Ihalainen, P.; Pulkkinen, P.; Tenhu, H.; Bobacka, J.; Peltonen, J. A Low-Cost Paper-Based Inkjet-Printed Platform for Electrochemical Analyses. Sens. Actuators, B 2013, 177, 153-162.

65. Ihalainen, P.; Majumdar, H.; Viitala, T.; Törngren, B.; Närjeoja, T.; Määttänen, A.; Sarfraz, J.; Härmä, H.; Yliperttula, M.; Österbacka, R.; Peltonen, J. Application of Paper-Supported Printed Gold Electrodes for Impedimetric Immunosensor Development. Biosensors 2013, 3, 1-17.

66. Hu, C.; Bai, X.; Wang, Y.; Jin, W.; Zhang, X.; Hu, S. Inkjet Printing of Nanoporous Gold Electrode Arrays on Cellulose Membranes for High-Sensitive Paper-Like Electrochemical Oxygen Sensors Using Ionic Liquid Electrolytes. Anal. Chem. 2012, 84, 3745-3750.

67. Grieshaber, T.; MacKenzie, R.; Voroz, J.; Reimhult, E. Electrochemical Biosensors - Sensor Principles and Architectures. Sensors (Basel) 2008, 8, 14001458 .

68. Raj, C. R.; Okajima, T.; Ohsaka, T. Gold nanoparticle Arrays for the Voltammetric Sensing of Dopamine. J. Electroanal. Chem. 2003, 543, 127-133.

69. Xue, C.; Han, Q.; Wang, Y, Wu, J.; Wen, T.; Wang, R.; Hong, J.; Zhou, X.; Jiang, $\mathrm{H}$. Amperometric detection of dopamine in human serum by electrochemical sensor based on gold nanoparticles doped molecularly imprinted polymers. Biosens. Bioelectron. 2013, 49, 199-203.

70. Chandra, P.; Singh, J.; Singh, A.; Srivastava, A.; Goyal, R. N.; Shim, Y. B. Gold Nanoparticles and Nanocomposites in Clinical Diagnostics Using Electrochemical Methods. J. Nanoparticles 2013, 535901.

71. Ying, W. NAD+/NADH and NADP ${ }^{+} / \mathrm{NADPH}$ in Cellular Functions and Cell Death: Regulation and Biological Consequences. Antioxid. Redox Signal. 2008, 10, 179-206.

72. Belenky, P.; Bogan, K. L.; Brenner, C. NAD + metabolism in health and disease. Trends Biochem. Sci. 2007, 32, 12-19. 
73. Greenamyre, J. T.; Sherer, T. B.; Betarbet, R.; Panov, A. V. Complex I and Parkinson's disease. IUBMB Life 2001, 52, 135-141.

74. Heikal, A. A. Intracellular Coenzymes as Natural Biomarkers for Metabolic Activities and Mitochondrial Anomalies. Biomark. Med. 2010, 4, 241-263.

75. Singer, T. P.; Ramsay, R. R.; Ackrell, B. A. C. Deficiencies of NADH and Succinate Dehydrogenases in Degenerative Diseases and Myopathies. Biochimica et. Biophysica Acta 1995, 1271, 211-219.

76. Hroudov, J.; Singh, N.; Fišar, Z. Mitochondrial Dysfunctions in Neurodegenerative Diseases: Relevance to Alzheimer's Disease. Biomed. Res. Int. 2014, 2014, 175092.

77. Xie, W. J.; Xu, A. S.; Yeung, E. S. Determination of $\mathrm{NAD}^{+}$and NADH in a Single Cell under Hydrogen Peroxide Stress by Capillary Electrophoresis. Anal. Chem. 2009, 81, 1280-1284.

78. Heikal, Yu. J. S.; Guo, H. W.; Wang, C H.; Wei, Y. H.; Wang, H. W. Increase of Reduced Nicotinamide Adenine Dinucleotide Fluorescence Lifetime Precedes Mitochondrial Dysfunction in Staurosporine-Induced Apoptosis of HeLa Cells. $J$. Biomed. Opt. 2011, 3:036008.

79. Jonas, S. K.; Benedetto, C.; Flatman, A.; Hammond, R. H.; Micheletti, L.; Riley, C.; Riley, P. A.; Spargo, D. J.; Zonca, M.; Slater, D. F. Increased Activity of 6phosphogluconate dehydrogenase and Glucose-6-phosphate Dehydrogenase in Purified Cell Suspensions and Single Cells From the Uterine Cervix in Cervical Intraepithelial Neoplasia. J. Cancer 1992, 66, 185-191.

80. Birkmayer, J. G. D.; Vrecko, C.; Volc, D.; Birkmayer, W. Nicotinamide Adenine Dinucleotide (NADH)- A New Therapeutic Approach to Parkinson's Disease. Comparison of Oral and Parenteral Application. Acta Neurol. Scand. 1993, 146, 32-35.

81. Williamson, D. H.; Lund, P.; Krebs, H. A. The Redox State of Free NicotinamideAdenine Dinucleotide in the Cytoplasm and Mitochondria of Rat Liver. Biochem $J .1967,103,514-527$.

82. Gray, J. P.; Eisen, T.; Cline, G. W.; Smith, P. J. S.; Heart, E. Plasma Membrane Electron Transport in Pancreatic $\beta$-Cells is Mediated in Part by NQO1. Am. J. Physiol. Endocrinol. Metab. 2011, 301, E113-E121.

83. Liu, Z. H.; Cai, R. X.; Wang, J. Current Development in the Determination of Intracellular NADH Level. Reviews in Fluorescence 2006, 107-124. 
84. Ke, U.; Hideo, K. Determination of Oxidized and Reduced Nicotinamide Adenine Dinucleotide in Cell Monolayers Using a Single Extraction Procedure and a Spectrophotometric Assay. Anal. Biochem. 2005, 338, 131-135.

85. Sporty, J. L.; Kabir, M.; Turteltaub, K. L.; Ognibene, T.; Lin, S. J.; Bench, G. Single Sample Extraction Protocol for the Quantification of NAD and NADH Redox States in Saccharomyces cerevisiae. J. Sep. Sci. 2008, 31, 3202-3211.

86. McComb, R. B.; Bond, L. W.; Burnett, R. W.; Keech, R. C.; Bowers, G. N. Jr. Determination of the Molar Absorptivity of NADH. Clin. Chem. 1976, 22, 141150.

87. Ochab, M. K.; Rodak, M. B.; Duda, E. Z.; Lejczak, B.; Kafarski, P. Comparative Study of Fungal Cell Disruption-Scope and Limitations of the Methods. Folia. Microbiol.(Praha) 2011, 56, 469-475.

88. Voulgaris, I.; O’Donnell, A.; Harvey, L. M.; McNeil, B. Inactivating Alternative NADH Dehydrogenases: Enhancing Fungal Bioprocesses by Improving Growth and Biomass Yield? Sci. Rep. 2012, 2, 322.

89. Wróbel, A. B.; Błażejak, S.; Kawarska, A.; Różańska, L. S.; Gientka, I.; Majewska, E. Evaluation of the Efficiency of Different Disruption Methods on Yeast Cell Wall Preparation for $\beta$-Glucan Isolation. Molecules 2014, 19, 2094120961.

90. Hu, T. X.; Yu, M.; Zhao, J. Comparative Transcriptional Profiling Analysis of the Two Daughter Cells from Tobacco Zygote Reveals the Transcriptome Differences in the Apical and Basal Cells. BMC Plant Biol. 2010, 10, 167.

91. Zhao, Y.; Jin, J.; Hu, Q.; Zhou, H. M.; Yi, J.; Yu, Z.; Xu, L.; Wang, X.; Yang, Y.; Loscalzo, J. Genetically Encoded Fluorescent Sensors for Intracellular NADH Detection. Cell Metab. 2011, 14, 555-566.

92. Candeias, L. P.; MacFarlane, D. P. S.; McWhinnie, S. L. W.; Maidwell, N. L.; Roeschlaub, C. A.; Sammes, P. G.; Whittlesey, R. The catalysed NADH Reduction of Resazurin to Resorufin. J. Chem. Soc., Perkin Trans. 1998, 2, $2333-$ 2334.

93. Votyakova, T. V.; Reynolds, I. J. Detection of hydrogen peroxide with Amplex Red: interference by NADH and reduced glutathione auto-oxidation. Arch. Biochem. Biophys. 2004, 431, 138-144.

94. Moore, F. M.; Simpson, D. Detection of Benzoylecgonine (Cocaine Metabolite) in Urine: A Cost-Effective Low Risk Immunoassay Procedure. Med. Lab. Sci. 1990, 47, 85-89. 
95. Yan, F.; Zheng, W.; Yao, L.; Su, B. Direct Electrochemical Analysis in Complex Samples Using ITO Electrodes Modified with Permselective Membranes Consisting of Vertically Ordered Silica Mesochannels and Micelles. Chem. Commun. 2015, 51, 17736-17739.

96. Harper, J. C.; Polsky, R.; Dirk, S. M.; Wheeler, D. R.; Brozik, S. M. Electroaddressable Selective Functionalization of Electrode Arrays: Catalytic NADH Detection Using Aryl Diazonium Modified Gold Electrodes. Electroanalysis 2007, 19, 1268-1274.

97. Song, H. K.; Lee, S. H.; Won, K.; Park, J. H.; Kim, J. K.; Lee, H.; Moon, S. J.; Kim, D. K.; Park, C. B. Electrochemical Regeneration of NADH Enhanced by Platinum Nanoparticles. Angew. Chem., Int. Ed. 2008, 47, 1749-1752.

98. Hassan, K. M.; Hathoot, A. A.; Ashour, W. F. D.; Abdel-Azeem, M. Electrochemical and Analytical Applications for NADH Detection at Glassy Carbon Electrode Modified with Nickel Nanoparticles Dispersed on poly 1,5Diaminonaphthalene. J. Solid State Electrochem. 2015, 19, 1063-1072.

99. Yao, H.; Halsall, H. B.; Heinman, W. R.; Jenkins, S. H. Electrochemical dehydrogenase-based homogeneous assays in whole blood. Clin. Chem. 1995, 41, 591-598.

100. Istrate, O. M.; Rotariu, L.; Marinescu, V. E.; Bala, C. NADH Sensing Platform Based on Electrochemically Generated Reduced Graphene Oxide-Gold Nanoparticles Composite Stabilized with Poly(allylamine hydrochloride). Sens. Actuators, B 2016, 223, 697-704.

101. Ahammad, A. J. S.; Lee, J. J.; Rahman, A. Electrochemical Sensors Based on Carbon Nanotubes. Sensors 2009, 9, 2289-2319.

102.Deng, C.; Chen, J.; Chen, X. L.; Xiao, C.; Nie, Z.; Yao, S. Boron-doped Carbon Nanotubes Modified Electrode for Electroanalysis of NADH. Electrochem. Commun. 2008, 10, 907-909.

103.Eguilaz, M.; Agüí, L.; Yáñez-Sedeño P.; Pingarrón, J. M. A Biosensor Based on Cytochrome c Immobilization on a Poly-3-methylthiophene/Multi-Walled Carbon Nanotubes Hybrid-Modified Electrode. Application to the Electrochemical Determination of Nitrite. J. Electroanal. Chem. 2010, 644, 3035 .

104.Wu, Y.; Lin, X.; Zhang, M. Carbon Nanotubes for Thin Film Transistor: Fabrication, Properties, and Applications. J. Nanomater. 2013, 627215. 
105. Arugula, M.; Simonian, A. Review-Nanocarbon-Based Multi-Functional Biointerfaces: Design and Applications. ECS J. Solid State Sci. Technol. 2016, 5, M3045-M3053.

106. Lalaoui, N.; Means, N.; Walgama, C.; Goff, A. L.; Holzinger, M.; Krishnan, S.; Cosnier, S. Enzymatic versus Electrocatalytic Oxidation of NADH at CarbonNanotube Electrodes Modified with Glucose Dehydrogenases: Application in a Bucky-Paper-Based Glucose Enzymatic Fuel Cell. ChemElectroChem 2016, 3, 2058-2062.

107. Deng, L.; Young, R. J.; Kinloch, I. A.; Abdelkader, A. R.; Holmes, S. M.; Rio, D. A. D.; Eichhorn, S. J. Supercapacitance from Cellulose and Carbon Nanotube Nanocomposite Fibers. ACS Appl. Mater. Interfaces, 2013, 5, 99839990.

108. Loper, K. A. Clinical Toxicology of Cocaine. Med. Toxicol. Adverse Drug Exp. 1989, 4, 174-185.

109. Center for Behavioral Health Statistics and Quality. (2015). Behavioral health trends in the United States: Results from the 2014 National Survey on Drug Use and Health (HHS Publication No. SMA 15-4927, NSDUH Series H-50.

110. Avois, L.; Robinson, N.; Saudan, C.; Baume, N.; Saugy, M. Central Nervous System Stimulants and Sport Practice. Br. J. Sports Med. 2006, 40, i16-i20.

111. Martin-Schild, S.; Albright, K. C.; Hallevi, H.; Barreto, A. D.; Philip, M.; Misra, V.; Grotta, J. C.; Savitz, S. I. Intracerebral Hemorrhage in Cocaine Users. Stroke 2010, 41, 680-684.

112.DiGregorio, G. J.; Ferko, A. P.; Barbieri, E. J.; Ruch, E. K.; Chawla, H.; Keohane, D.; Rosenstock, R.; Aldano, A. Determination of Cocaine Usage in Pregnant Women by a Urinary EMIT Drug Screen and GC-MS Analyses. $J$. Anal. Toxicol. 1994, 18, 247-250.

113. Moody, D. E.; Spanbauer, A. C.; Taccogno, J. L.; Smith, E. K. Comparative Analysis of Sweat Patches for Cocaine (and Metabolites) by Radioimmunoassay and Gas Chromatography-Positive Ion Chemical Ionization-Mass Spectrometry. J. Anal. Toxicol. 2004, 28, 86-93.

114. Marchei, E.; Colone, P.; Nastasi, G. G.; Calabrò, C.; Pellegrini, M.; Pacifici, R.; Zuccaro, P.; Pichini, S. On-site Screening and GC-MS Analysis of Cocaine and Heroin Metabolites in Body-Packers Urine. J. Pharm. Biomed. Anal. 2008, 48, 383-387.

115.López, P.; Martello, S.; Bermejo, A. M.; De Vincenzi, E.; Tabernero, M. J.; Chiarotti, M. Validation of ELISA Screening and LC-MS/MS Confirmation 
Methods for Cocaine in Hair After Simple Extraction. Anal. Bioanal. Chem. 2010, 397, 1539-1548.

116. Moeller, K. E.; Lee, K. C.; Kissack, J. C. Urine Drug Screening: Practical Guide for Clinicians. Mayo Clin. Proc. 2008, 83, 66-76.

117.Phillips, D. L.; Tebbett, I. R.; Bertholf, R. L. Comparison of HPLC and GC-MS for Measurement of Cocaine and Metabolites in Human Urine. J. Anal. Toxicol. 1996, 20, 305-308.

118. Kapur, B. M. Drug-Testing Methods and Clinical Interpretations of Test Results. Bull. Narc. 1993, 45, 115-154.

119. Cone, E. J. Validity Testing of Commercial Urine Cocaine Metabolite assays: III. Evaluation of an Enzyme-Linked Immunosorbent Assay (ELISA) for Detection of Cocaine and Cocaine Metabolite. J. Forensic Sci. 1989, 34, 991995.

120.López, P.; Martello, S.; Bermejo, A. M.; De Vincenzi, E.; Tabernero, M. J.; Chiarotti, M. Validation of ELISA Screening and LC-MS/MS Confirmation Methods for Cocaine in Hair After Simple Extraction. Anal. Bioanal. Chem. 2010, 397, 1539-1548.

121. Armbruster, D. A.; Schwarzhoff, R. H.; Hubster, E. C.; Liserio, M. K. Enzyme Immunoassay, Kinetic Microparticle Immunoassay, Radioimmunoassay, and Fluorescence Polarization Immunoassay Compared for Drugs-of-Abuse Screening. Clin. Chem. 1993, 39, 2137-2146.

122. Thivianathan, V.; Gorenstein, D. G. Aptamers and the Next Generation of Diagnostic Reagents. Poteomics Cin. Appl. 2012, 6, 563-573.

123. Tuerk, C.; Gold, L. Systematic Evolution of Ligands by Exponential Enrichment: RNA Ligands to Bacteriophage T4 DNA Polymerase. Science 1990, 249, 505510 .

124.Zhang, P.; Zhao, N.X.; Zeng, Z. H.; Chang, C. C.; Zu, Y. L. Combination of an Aptamer Probe to CD4 and Antibodies for Multicolored Cell Phenotyping. Am. J. Clin. Pathol. 2010, 134, 586-593.

125. Woo, H. M.; Kim, K. S.; Lee, J. M.; Shim, H. S.; Cho, S. J.; Lee, W. K.; Ko, H. W.; Keum, Y. S.; Kim, S. Y.; Pathinayake, P.; Kim, C. J.; Jeong, Y. J. SingleStranded DNA Aptamer that Specifically Binds to the Influenza Virus NS1 Protein Suppresses Interferon Antagonism. Antiviral Res. 2013, 100, 337-345. 
126.Zhou, J. H.; Li, H. T.; Li, S.; Zaia, J.; Rossi, J. J. Novel Dual Inhibitory Function AptamersiRNA Delivery System for HIV-1 Therapy. Mol. Ther. 2008, 16, 14811489.

127. Sefah, K.; Shangguan, D. H.; Xiong, X. L.; O'Donoghue, M. B.; Tan, W. H. Development of DNA Aptamers Using Cell-SELEX. Nat. Protoc. 2010, 5, 11691185.

128. Tan, W.H.; Donovan, M. J.; Jiang, J. H. Aptamers from Cell-Based Selection for Bioanalytical Applications. Chem. Rev. 2013, 113, 2842-2862.

129. Chen, H. W.; Medley, C. D.; Sefah, K.; Shangguan, D.; Tang, Z. W.; Meng, L.; Smith, J. E.; Tan, W. H. Molecular Recognition of Small-Cell Lung Cancer Cells Using Aptamers. Chem. Med. Chem. 2008, 3, 991-1001.

130. Shangguan, D. H,; Meng, L.; Cao, Z. C.; Xiao, Z. Y.; Fang, X. H.; Li, Y.; Cardona, D.; Witek, R. P.; Liu, C.; Tan, W. H. Identification of Liver CancerSpecific Aptamers Using Whole Live Cells. Anal. Chem. 2008, 80, 721-728.

131. Stojanovic, M. N.; de Prada, P.; Landry, D. W. Fluorescent Sensors Based on Aptamer Self- Assembly. J. Am. Chem. Soc. 2000, 122, 11547-11548.

132. Stojanovic, M. N.; de Prada, P.; Landry, D. W. Aptamer-Based Folding Fluorescent Sensor for Cocaine. J. Am. Chem. Soc. 2001, 123, 4928-4931.

133. Rajendran, M.; Ellington, A. D. Selection of Fluorescent Aptamer Beacons that Light up in the Presence of Zinc. Anal. Bioanal. Chem. 2008, 390, 1067-1075.

134. Mok, W.; Li, Y. F. Recent Progress in Nucleic Acid Aptamer-Based Biosensors and Bioassays. Sensors 2008, 8, 7050-7084.

135. Oh, S. S.; Plakos, K.; Lou, Xinhui.; Xiao, Y.; Soh, H. T. In vitro Selection of Structure-Switching, Self-Reporting Aptamers. Proc. Natl. Acad. Sci. U. S. A. 2010, 107, 14053-14058.

136.Ebrahimi, M.; Johari-Ahar, M.; Hamzeiy, H.; Barar, J.; Maschinchian, O.; Omidi, Y. Electrochemical Impedance Spectroscopic Sensing of Methamphetamine by a Specific Aptamer. Bioimpacts 2012, 2, 91-95.

137. Ramezani, M.; Danesh, M. N.; Lavaee, P.; Abnous, K.; Taghdsisi, S. M. A Novel Colorimetric Triple-Helix Molecular Switch Aptasensor for Ultrasensitive Detection of Tetracycline. Biosens. Bioelectron. 2015, 70, 181-187.

138. Xia, F.; Zuo, X.; Yang, R.; Xiao, Y.; Kang, D.; Belisle, A. V.; Gong, X.; Yuen, J. D.; Hsu, B. B. Y.; Heeger, A. J.; Plaxco, K. W. Colorimetric detection of DNA, small molecules, proteins, and ions using unmodified gold nanoparticles 
and conjugated polyelectrolytes. Proc. Natl. Acad. Sci. U. S. A. 2010, 107, 10837-10841.

139.Sun, B.; Qi, H.; Ma, F.; Gao, Q.; Zhang, C.; Miao, W. Double Covalent Coupling Method for the Fabrication of Highly Sensitive and Reusable Electrogenerated Chemiluminescence Sensors. J. Am. Chem. Soc. 2010, 82, 5046-5052.

140. White, R. J.; Plaxco, K. W. Engineering New Aptamer Geometries for Electrochemical Aptamer-Based Sensors. Proc. SPIE Int. Soc. Opt. Eng. 2009, $7321: 732105$.

141. Chiu, T. C.; Huang, C. C. Aptamer-Functionalized Nano-Biosensors. Sensors (Basel) 2009, 9, 10356-10388.

142. Stojanovic, M. N.; Landry, D. W. Aptamer-Based Colorimetric Probe for Cocaine. Am. Chem. Soc. 2002, 124, 9678-9679.

143.Zou, R. X.; Lou, X. H.; Ou, H. C.; Zhang, Y.; Wang, W. J.; Yuan, M.; Guan, M.; Luo, Z. F.; Liu, Y. Y. Highly Specific Triple-Fragment Aptamer for Optical Detection of Cocaine. RSC Adv. 2012, 2, 4636-4638.

144.Zhao, T.; Liu, R.; Ding, X. F.; Zhao, J. C.; Yu, H. X.; Wang, L.; Xu, Q.; Wang, X.; Lou, X. H.; He, M.; Xiao, Y. Nanoprobe-Enhanced, Split Aptamer-Based Electrochemical Sandwich Assay for Ultrasensitive Detection of Small Molecules. Anal. Chem. 2015, 87, 7712-7719.

145. Freeman, R.; Li, Y.; Tel-Vered, R.; Sharon, E.; Elbaz, J.; Willner, I. SelfAssembly of Supramolecular Aptamer Structures for Optical or Electrochemical Sensing. Analyst 2009, 134, 653-656.

146.Du, Y.; Li, B. L.; Guo, S. J.; Zhou, Z. X.; Zhou, M.; Wang, E. K.; Dong, S. J. GQuadruplex-Based DNAzyme for Colorimetric Detection of Cocaine: Using Magnetic Nanoparticles as the Separation and Amplification Element. Analyst 2011, 136, 493-497.

147.Zhang, J.; Wang, L. H.; Pan, D.; Song, S. P.; Boey, F. Y. C.; Zhang, H.; Fan, C. H. Visual Cocaine Detection with Gold Nanoparticles and Rationally Engineered Aptamer Structures. Small 2008, 4, 1196-1200.

148. Liu, J.; Lu, Y. Fast Colorimetric Sensing of Adenosine and Cocaine Based on a General Sensor Design Involving Aptamers and Nanoparticles. Angew. Chem., Int. Ed. 2006, 45, 90-94. 
149.Perutz, M. F.; Wilkinson, A. J.; Paoli, M.; Dodson, G. G. The Stereochemical Mechanism of the Cooperative Effects in Hemoglobin Revisited. Annu. Rev. Biophys. Biomol. Struct. 1998, 27, 1-34.

150. Meyer, T.; Holowka, D.; Stryer, L. Highly Cooperative Opening of Calcium Channels by Inositol 1,4,5-Trisphosphate. Science 1988, 240, 653-656.

151.Krell, T.; Ter'an, W.; Mayorga, O. L.; Rivas, G.; Jim'enez, M.; Daniels, C.; Molina-Henares, A. J.; Mart'inez-Bueno, M.; Gallegos, M. T.; Ramos, J. L. J. Mol. Biol. 2007, 369, 1188-1199.

152. Mandall, M.; Lee, M.; Barrick, J. E.; Weinberg, Z.; Emilsson, G. M.; Ruzzo, W. L.; Breaker, R. R. A Glycine-Dependent Riboswitch that uses Cooperative Binding to Control Gene Expression. Science 2004, 306, 275-279.

153. Sudarsan, N.; Hammond, M. C.; Block, K. F.; Welz, R.; Barrick, J. E.; Roth, A.; Breaker, R. R. Tandem Riboswitch Architectures Exhibit Complex Gene Control Functions. Science 2006, 314, 300-304.

154. Jose, A. M.; Soukup, G. A.; Breaker, R. R. Cooperative Binding of Effectors by an Allosteric Ribozyme. Nucleic Acids Res. 2001, 29, 1631-1637.

155. Simon, A. J.; Vall'ee-B'elisle, A.; Ricci, F.; Watkins, H. M.; Plaxco, K. W. Using the Population-Shift Mechanism to Rationally Introduce "Hill-type" Cooperativity into a Normally Non-Cooperative Receptor. Angew. Chem., Int. Ed., 2014, 53, 9471-9475.

156. Yu, H. X.; Canoura, J.; Guntupalli, B.; Lou, X; Xiao, Y. A Cooperative-Binding Split Aptamer Assay for Rapid, Specific and Ultra-Sensitive Fluorescence Detection of Cocaine in Saliva. Chem. Sci. 2017, 8, 131-141.

157. Glynn, C.; O'Dwyer, C. Solution Processable Metal Oxide Thin Film Deposition and Material Growth for Electronic and Photonic Devices. Adv. Mater. Interfaces 2017, 4, 1600610.

158. Valden, M.; Pak, S.; Lai, X.; Goodman, D. W. Structure Sensitivity of CO Oxidation over Model Au/TiO2 Catalysts. Catal. Lett. 1998, 56, 7-10.

159. Rho, S.; Jahng, D.; Lim, J. H.; Choi, J.; Chang, J. H.; Lee, S. C.; Kim, K. J. Electrochemical DNA Biosensors Based on Thin Gold Films Sputtered on Capacitive Nanoporous Niobium Oxide. Biosens. Bioelectron. 2008, 23, 852-856.

160. Hertz, J. L.; Tuller, H. L. Electrochemical Characterization of Thin Films for a Micro-Solid Oxide Fuel Cell. J. Electroceram. 2004, 13, 663-668. 
161.Ku, K.; Kim, B.; Chung, H.; Kim, W. Characterization of Graphene-Based Supercapacitors Fabricated on Al Foils Using $\mathrm{Au}$ or Pd Thin Films as Interlayers. Synth. Met. 2010, 160, 2613-2617.

162.Liang, K. S.; Salaneck, W. R.; Aksay, I. A. X-ray Photoemission Studies of Thin Gold Films. Solid State Commun. 1976, 19, 329-334.

163. Chen, G.; Hui, P. Thermal Conductivities of Evaporated Gold Films on Silicon and Glass. Appl. Phys. Lett. 1999, 74, 2942-2944.

164. Anandan, V.; Yang, X.; Kim, E.; Rao, Y. L.; Zhang, G. Role of Reaction Kinetics and Mass Transport in Glucose Sensing with Nanopillar Array Electrodes. J. Biol. Eng. 2007, 1, 5.

165. Bishop, P. T.; Ashfield, L. J.; Berzins, A.; Boardman, A.; Buche, V.; Cookson, J.; Gordon, R. J.; Salcianu, C.; Sutton, P. A. Printed Gold for Electronic Applications. Gold Bull. (Berlin, Ger.) 2010, 43, 181-188.

166. Frens, G. Controlled Nucleation for the Regulation of the Particle Size in Monodisperse Gold Suspensions. Nature (London), Phys. Sci. 1973, 241, 20-22.

167. Bastús, N. G.; Comenge, J.; Puntes, V. Kinetically Controlled Seeded Growth Synthesis of Citrate-Stabilized Gold Nanoparticles of up to $200 \mathrm{~nm}$ : Size Focusing versus Ostwald Ripening. Langmuir 2011, 27, 11098-11105.

168. Haiss, W.; Thanh, N. T. K.; Aveyard, J.; Fernig, D. G. Determination of Size and Concentration of Gold Nanoparticles from UV-Vis Spectra. Anal. Chem. 2007, $79,4215-4221$.

169. Siqueira, J. R.; Gabriel, R. C.; Zucolotto, V.; Silva, A. C.; Dantas, N. O.; Gasparotto, L. H. Electrodeposition of Catalytic and Magnetic Gold Nanoparticles on Dendrimer-Carbon Nanotube Layer-by-Layer Films. Phys. Chem. Chem. Phys. 2012, 14, 14340-14343.

170. Angerstein-Kozlowska, H.; Conway, B. E.; Hamelin, A.; Stoicoviciu, L. Elementary Steps of Electrochemical Oxidation of Single-Crystal Planes of Au Part II. A Chemical and Structural Basis of Oxidation of the (111) Plane. $J$. Electroanal. Chem. Interfacial Electrochem. 1987, 228, 429-453.

171. Nicholson, R. S. Theory and Application of Cyclic Voltammetry for Measurement of Electrode Reaction Kinetics. Anal. Chem. 1965, 37, 1351-1355.

172. Kumar, B. R.; Rao, T. S. AFM Studies on Surface Morphology, Topography and Texture of Nanostructured Zinc Aluminium Oxide Thin Films. Dig. J. Nanomater. Bios. 2012, 7, 1881-1889. 
173. Thomas, T. Rough Surfaces, 2nd ed.; Imperial College Press: London, 2009.

174.Wu, Z.; Chen, Z.; Du, X.; Logan, J. M.; Sippel, J.; Nikolou, M.; Kamaras, K.; Reynolds, J. R.; Tanner, D. B.; Hebard, A. F.; Rinzler, A. G. Transparent, Conductive Carbon Nanotube Films. Science 2004, 305, 1273-1276.

175.Eda, G.; Fanchini, G.; Chhowalla, M. Large-Area Ultrathin Films of Reduced Graphene Oxide as a Transparent and Flexible Electronic Material. Nat. Nanotechnol. 2008, 3, 270-274.

176.Xiao, Y.; Lai, R. Y.; Plaxco, K. W. Preparation of Electrode- Immobilized, Redox-Modified Oligonucleotides for Electrochemical DNA and Aptamer-Based Sensing. Nat. Protoc. 2007, 2, 2875-2880.

177.Zhou, J.; Sheng, M.; Jiang, X.; Wu, G.; Gao, F. Simultaneous Determination of Dopamine, Serotonin and Ascorbic Acid at a Glassy Carbon Electrode Modified with Carbon-Spheres. Sensors 2013, 13, 14029-14040.

178. Park, S.; Kim, H. C.; Chung, T. D. Electrochemical Analysis Based on Nanoporous Structures. Analyst 2012, 137, 3891-3903.

179. Qiu, H. J.; Zhou, G. P.; Ji, G. L.; Zhang, Y.; Huang, X. R.; Ding, Y. A Novel Nanoporous Gold Modified Electrode for the Selective Determination of Dopamine in the Presence of Ascorbic Acid. Colloids Surf., B 2009, 69, 105-108.

180.El-Said, W. A.; Lee, J. H.; Oh, B. K.; Choi, J. W. 3-D Nanoporous Gold Thin Film for the Simultaneous Electrochemical Determination of Dopamine and Ascorbic Acid. Electrochem. Commun. 2010, 12, 1756-1759.

181.Bard, A. J.; Faulkner, L. R. Electrochemical Methods: Fundamentals and Applications, 2nd ed.; Wiley: New York, 2001; 452.

182. Swamy, B. E. K.; Venton, B. J. Carbon Nanotube-Modified Microelectrodes for Simultaneous Detection of Dopamine and Serotoninin In vivo. Analyst 2007, $132,876-884$.

183. Venton, B. J.; Wightman, R. M. Psychoanalytical Electrochemistry: Dopamine and Behavior. Anal. Chem. 2003, 75, 414A-421A.

184. Wrona, M. Z.; Dryhurst, G. Electrochemical Oxidation of 5- Hydroxytryptamine in Acidic Aqueous Solution. J. Org. Chem. 1989, 54, 2718-2721.

185. Palau, F.; Espinos, C. Autosomal Recessive Cerebellar Ataxias. Orphanet J. Rare Dis. 2006, 1:47. 
186. Wagner, G. R.; Pride, P. M.; Babbey, C. M.; Payne, R. M. Friedreich's Ataxia Reveals a Mechanism for Coordinate Regulation of Oxidative Metabolism via Feedback Inhibition of the SIRT3 Deacetylase. Hum. Mol. Genet. 2012, 21, 2688-2697.

187.Zhang, Q.; Piston, D. W.; Goodman, R. H. Regulation of Corepressor Function by Nuclear NADH. Science 2002, 295,1895-1897.

188. Al-Ali, A. K. Pyridine Nucleotide Redox Potential in Erythrocytes of Sandi Subjects with Sickle Cell Disease. Acta Haematol. 2002, 108,19-22.

189. Wagner, T. C.; Scott, M. D. Single Extraction Method for the Spectrophotometric Quantification of Oxidized and Reduced Pyridine Nucleotides in Erythrocytes. Anal.Biochem. 1994, 222, 417-426.

190.Zerez, C. R.; Lee, S. J.; Tanaka, K. Spectrophotometric Determination of Oxidized and Reduced Pyridine Nucleotides in Erythrocytes. Anal. Biochem. 1987, 164, 367-373.

191. Lane, D. A.; Nadeau, D. Determination of Pyridine Nucleotide Contents of Cell Monolayers by Bioluminescence, J. Biochem. Biophys. Method 1988, 17,107118.

192. Chen, Y. H.; Cai, R. X. Determination of NADH with Inhibited Fluorometry. Chin. J. Anal. Chem. 2004, 32, 719.

193.Zhou, M.; Diwu, Z.; Panchuk, V. N.; Haugland, R. P. A Stable Nonfluorescent Derivative of Resorufin for the Fluorometric Determination of Trace Hydrogen Peroxide: Applications in Detecting the Activity of Phagocyte NADPH Oxidase and Other Oxidases. Anal. Biochem. 1997, 253, 162-168.

194. Maidwell, N. L.; Rezai, M. R.; Roeschlaub, C. A. On The Development of NAD(P)H-sensitive Fluorescent Probes. J. Chem. Soc. Perkin Trans. 2000, 1, 1541-1546.

195. Piston, D. W.; Knobel, S. M. Real-Time Analysis of Glucose, Metabolism by Microscopy. Trends Endocrinol. Metab. 1999, 10, 413-417.

196. Nieminen, A. L.; Byme, A. M.; Herman, B.; Lemasters, J. J. Mitochondrial Permeability Transition in Hepatocytes Induced by t-BuOOH: NAD(P)H and Reactive Oxygen Species. Am. J. Physiol. Cell Physiol. 1997, 272, C1286C1294.

197. Masters, B. R.; So, P. T. C.; Gratton, E. Multiphoton Excitation Microscopy of in vivo Human Skin: Functional and Morphological Optical Biopsy Based on 
Three-Dimensional Imaging, Lifetime Measurements and Fluorescence Spectroscopy, Ann. NY Acad. Sci. 1998, 838, 58-67.

198. Ma, W.; Li, D. W.; Sutherland, T. C.; Li, Y.; Long, Y. T.; Chen, H. Y. Reversible Redox of NADH and NAD+ at a Hybrid Lipid Bilayer Membrane Using Ubiquinone. J. Am. Chem. Soc. 2011, 133, 12366-12369.

199. Ma, W.; Ying, Y. L.; Qin, L. X.; Gu, Z.; Zhou, H.; Li, D. W.; Sutherland, T. C.; Chen, H. Y.; Long, Y. T. Investigating Electron-Transfer Processes Using a Biomimetic Hybrid Bilayer Membrane System. Nat. Protoc. 2013, 8, 439-450.

200.Patterson, G. H.; Knobel, S. M.; Arkhammar, P.; Thastrup, O.; Piston, D. W. Separation of the Glucose-Stimulated Cytoplasmic and Mitochondrial NAD(P)H Responses in Pancreatic Islet $\beta$ Cells. Proc. Natl. Acad. Sci. 2000, 97, 52035207.

201. Santos, A. S.; Pereira, A. C.; Kubota, L. T. Electrochemical and Electrocatalytic Studies of Toluidine Blue Immobilized on a Silica Gel Surface Coated with Niobium Oxide. J. Braz. Chem. Soc. 2002, 13, 495-501.

202. Kumar, S. A.; Chen, S. M. Electroanalysis of NADH Using Conducting and Redox Active Polymer/Carbon Nanotubes Modified Electrodes-A Review. Sensors (Basel) 2008, 8, 739-766.

203. Behera, S.; Raj, C. R. Self-Assembled Monolayers of Thio-Substituted Nucleobases on Gold Electrode for the Electroanalysis of NADH, Ethanol and Uric Acid. Sens. Actuators, B 2007, 128, 31-38.

204. Hoyer, B.; Jensen, N. Use of Sodium Dodecyl Sulfate for Suppression of Electrode Fouling in the Voltammetric Detection of Biologically Relevant Compounds. Electroanalysis 2005, 17, 2037-2042.

205. Raus, A. L.; Patmore, J.; Kurzepa, L.; Bulmer, J.; Koziol, K. Electrical Properties of Carbon Nanotube Based Fibers and Their Future Use in Electrical Wiring. Adv. Funct. Mater. 2014, 24, 3661-3682.

206. Lin, Y.; Yantasee, W.; Wang, J. Carbon Nanotubes (CNTs) for the Development of Electrochemical Biosensors. Front Biosci. 2005, 10, 492-505.

207. Milczarek, G.; Ciszewski, A. Voltammetric Detection of NADH Free from Interference of Ascorbic Acid Using a Glassy Carbon Electrode Modified with an Electropolymerized Porphyrin Film. Electroanalysis 2001, 13, 161-166.

208. Chen, G.; Guo, Z.; Zeng, G.; Tang, L. Fluorescent and Colorimetric Sensors for Environmental Mercury Detection. Analyst 2015, 140, 5400-5443. 
209. Stewart, M. E.; Anderton, C. R.; Thompson, L. B.; Maria, J.; Gray, S. K.; Rogers, J. A.; Nuzzo, R. G. Nanostructured Plasmonic Sensors. Chem. Rev. 2008, 108, 494-521.

210. Mokhtarzadeh, A.; Dolatabadi JE.; Abnous, K.; de la Guardia, M.; Ramezani, M. Nanomaterial-Based Cocaine Aptasensors. Biosens. Bioelectron. 2015, 68, 95106.

211. Jellinek, D.; Lynott, C. K.; Rifkin, D. B.; Janjić, N. High-Affinity RNA Ligands to Basic Fibroblast Growth Factor Inhibit Receptor Binding. Proc. Natl. Acad. Sci. U. S. A. 1993, 90, 11227-11231.

212.Sun, J.; Guo, A.; Zhang, Z.; Guo, L.; Xie, J. A Conjugated Aptamer-Gold Nanoparticle Fluorescent Probe for Highly Sensitive Detection of rHuEPO- $\alpha$. Sensors 2011, 11, 10490-10501.

213. Huang, C. C.; Chiu, S. H.; Huang, Y. F.; Chang, H. T. Aptamer-Functionalized Gold Nanoparticles for Turn-On Light Switch Detection of Platelet-Derived Growth Factor. Anal. Chem. 2007, 79, 4798-4804.

214.Zheng, D.; Seferos, D. S.; Giljohann, D. A.; Patel, P. C.; Mirkin, C. A. Aptamer nano-flares for molecular detection in living cells. Nano Lett. 2009, 9, 32583261 .

215. Mout, R.; Moyano, D. F.; Rana, S.; Rotello, V. M. Surface Functionalization of Nanoparticles for Nanomedicine. Chem. Soc. Rev. 2012, 41, 2539-2544.

216.Zu, Y. B.; Gao, Z. Q. Facile and Controllable Loading of Single-Stranded DNA on Gold Nanoparticles. Anal. Chem. 2009, 81, 8523-8528.

217. Cederquist, K. B.; Stoermer Golightly, R.; Keating, C. D. Molecular Beacon-Metal Nanowire Interface: Effect of Probe Sequence and Surface Coverage on Sensor Performance. Langmuir 2008, 24, 9162-9171.

218. Seferos, D. S.; Prigodich, A. E.; Giljohann, D. A.; Patel, P. C.; Mirkin, C. A. Polyvalent DNA Nanoparticle Conjugates Stabilize Nucleic Acids. Nano Lett. 2009, 9, 308-311.

219. Liu, C. W.; Huang, C. C.; Chang, H. T. Control over Surface DNA Density on Gold Nanoparticles Allows Selective and Sensitive Detection of Mercury (II). Langmuir 2008, 24, 8346-8350.

220. Hurst, S. J.; Lytton-Jean, A. K. R.; Mirkin, C. A. Maximizing DNA Loading on a Range of Gold Nanoparticle Sizes. Anal. Chem. 2006, 78, 8313-8318.

221. Seela, F.; Ding, P.; Budow, S. DNA Gold Nanoparticle Conjugates Incorporating 
Thiooxonucleosides: 7-Deaza-6-thio-2'-deoxyguanosine as Gold Surface Anchor. Bioconjugate Chem. 2011, 22, 794-807.

222.Demers, L. M.; Mirkin, C. A.; Mucic, R. C.; Reynolds, R. A.; Letsinger, R. L.; Elghanian, R.; Viswanadham, G. A Fluorescence-Based Method for Determining the Surface Coverage and Hybridization Efficiency of Thiol-Capped Oligonucleotides Bound to Gold Thin Films and Nanoparticles. Anal. Chem. 2000, 72, 5535-5541.

223. Law, B.; Moffat, A. C. The Evaluation of an Homogeneous Enzyme Immunoassay (Emit) and Radioimmunoassay for Barbiturates. J. Forensic Sci. Soc. 1981, 21, 55-66.

224. Popow, I.; Letiner, J.; Majdic, O.; Kovarik, J. J.; Seamann, M. D.; Zalbinger, G. J.; Steinberger, P. Assessment of Batch to Batch Variation in Polyclonal Antithymocyte Globulin Preparations. Transplantation 2012, 93, 32-40.

225. Mairal, T.; Ozalp, V. C.; Lozano S'anchez, P.; Mir, M.; Katakis, I.; O'Sullivan, C. K. Aptamers: Molecular Tools for Analytical Applications Anal. Bioanal. Chem. 2008, 390, 989-1007.

226. Baker, B. R.; Lai, R. Y.; Wood, M. S.; Doctor, E. H.; Heeger, A. J.; Plaxco, K. W. An Electronic, Aptamer-Based Small-Molecule Sensor for the Rapid, LabelFree Detection of Cocaine in Adulterated Samples and Biological Fluids. J. Am. Chem. Soc. 2006, 128, 3138-3139.

227. Sharma, A. K.; Kent, A. D.; Heemstra, J. M. Enzyme-Linked Small-Molecule Detection Using Split Aptamer Ligation. Anal. Chem. 2012, 84, 6104-6109.

228. Bray, D. Protein Molecules as Computational Elements in Living Cells. Nature 1995, 376, 307-312.

229. Elghanian, R.; Storhoff, J. J.; Mucic, R. C.; Letsinger, R. L.; Mirkin, C. A. Selective Colorimetric Detection of Polynucleotides Based on the DistanceDependent Optical Properties of Gold Nanoparticles. Science 1997, 277, 10781081.

230.Liu, J. W.; Lu, Y. Colorimetric $\mathrm{Cu}^{2+}$ Detection with a Ligation DNAzyme and Nanoparticles. Chem. Commun. 2007, 43, 4872-4874.

231. Jana, N. R.; Gearheart, L.; Murphy, C. J. Seeding Growth for Size Control of 5-40 nm Diameter Gold Nanoparticles. Langmuir 2001, 17, 6782-6786. 
232.Xiao, Y.; Pavlov, V.; Levine, S.; Niazov, T.; Markovitch, G.; Willner, I. Catalytic Growth of Au Nanoparticles by NAD(P)H Cofactors: Optical Sensors for NAD(P)+-Dependent Biocatalyzed Transformations. Angew. Chem. 2004, $116,4619-4622$.

233. Wang, L. H.; Liu, X. F.; Hu, X. F.; Song, S. P.; Fan, C. H. Unmodified Gold Nanoparticles as a Colorimetric Probe for Potassium DNA Aptamers. Chem. Commun. 2006, 42, 3780-3782.

234. Liz-Marzán, L. M. Tailoring Surface Plasmons through the Morphology and Assembly of Metal Nanoparticles. Langmuir 2006, 22, 32-41.

235.Jans, H.; Liu, X.; Austin, L.; Maes, G.; Huo, Q. Dynamic Light Scattering as a Powerful Tool for Gold Nanoparticle Bioconjugation and Biomolecular Binding Studies. Anal. Chem. 2009, 81, 9425-9432.

236. Roncancio, D.; Yu, H. X.; Xu, X. W.; Wu, S.; Liu, R.; Debord, J.; Lou, X. H.; Xiao, Y. A Label-Free Aptamer-Fluorophore Assembly for Rapid and Specific Detection of Cocaine in Biofluids. Anal. Chem. 2014, 86, 11100-11106.

237. Stojanovic, M. N.; de Prada, P.; Landry, D. W. Fluorescent Sensors Based on Aptamer Self Assembly. J. Am. Chem. Soc. 2000, 122, 11547-11548.

238. Wu, S.; Liang, P.P.; Yu, H. X; Xu, X. W; Liu, Y.; Lou, X. H; Xiao, Y. Amplified Single Base-Pair Mismatch Detection via Aggregation of Exonuclease-Sheared Gold Nanoparticles. Anal. Chem. 2014, 86, 3461-3467. 
VITA

\section{BHARGAV GUNTUPALLI}

Born, Andhra Pradesh, India

2003-2007

B.Sc., Pharmacy

G Pulla Reddy College of Pharmacy

Hyderabad, India

2008-2010

M.S., Analytical Chemistry

Governors State University

Chicago, Illinois

2011

Chemist

Catalent Pharma Solutions

Morrisville, North Carolina

2011-2015

Teaching Assistant

Florida International University

Miami, Florida

$2016-2017$

Dissertation Year Fellowship Award

Florida International University

Miami, Florida

2015-2017

Doctoral Candidate

Florida International University

Miami, Florida

\section{PUBLICATIONS AND PRESENTATIONS}

1. Yu H.X., Xu X.W., Liang P.P., Loh K.Y., Guntupalli B., Roncancio D. \& Xiao Y. A broadly applicable assay for rapidly and accurately quantifying DNA surface coverage on diverse particles. Bioconjugate Chem., 28, 933-943, 2017. (IF: 4.500). Time cited: 0

2. Yu H.X., Canoura J., Guntupalli B., Lou X.H. \& Xiao Y. A cooperative-binding split aptamer assay for rapid, specific and ultra-sensitive fluorescence detection of cocaine in saliva. Chem. Sci., 8, 131-141, 2016. (IF: 9.144). Time cited: 3.

3. Guntupalli B., Liang P.P., Lee J.H., Yang Y.H., Yu H.X., Canoura J., He J., Li W.Z., Weizmann Y. \& Xiao Y. Ambient filtration method to rapidly prepare highly conductive, paper-based porous gold films for electrochemical biosensing. ACS Appl. Mater. Interfaces. 7, 27049 - 27058, 2015. (IF: 7.145). Time cited: 0. 
4. Liang P.P., Yu H.X., Guntupalli B. \& Xiao Y. Paper-based device for rapid visualization of NADH based on dissolution of gold nanoparticles. ACS Appl. Mater. Interfaces. 7, 15023 - 15030, 2015. (IF: 7.145). Time cited: 11.

5. Xiao Y., Guntupalli B. \& Paudyal J. "Highly conductive porous paper-based metal thin films". Patent No. US20160329121 A1.

6. Yu H.X., Guntupalli B. \& Xiao Y. (2016) A cooperative-binding split aptamer assay for rapid, specific and ultra-sensitive fluorescence detection of cocaine in saliva. Oral presentation, 2016 Conference of Undergraduate Research at FIU (CURFIU), Miami, FL, March $30-31$.

7. Guntupalli B., Liang P.P., Lee J.H., Yang Y.H., Yu H.X., Canoura J., He J., Li W.Z., Weizmann Y. \& Xiao Y. (2016) Ambient filtration method to rapidly prepare highly conductive, paper-based porous gold films for electrochemical biosensing. Oral presentation, 2016 Conference of Undergraduate Research at FIU (CURFIU), Miami, FL, March 30 - 31.

8. Liang P.P., Yu H.X. Guntupalli B. \& Xiao Y. (2016) Paper-based device for rapid visualization of $\mathrm{NADH}$ based on dissolution of gold nanoparticles. Oral presentation, 2016 Conference of Undergraduate Research at FIU (CURFIU), Miami, FL, March $30-31$.

9. Liang P.P., Yu H.X., Guntupalli B. \& Xiao Y. (2016) Paper-based device for rapid visualization of NADH based on dissolution of gold nanoparticles. Poster presentation, FIU's annual Scholarly Forum during Graduate Student Appreciation Week (GSAW), Florida International University, Miami, FL, March $28-29,2016$.

10. Yu H.X., Guntupalli B. \& Xiao Y. (2016) A cooperative-binding split aptamer assay for rapid, specific and ultra-sensitive fluorescence detection of cocaine in saliva. Oral presentation, FIU's annual Scholarly Forum during Graduate Student Appreciation Week (GSAW), Florida International University, Miami, FL, March $28-29,2016$.

11. Guntupalli B., Liang P.P., Lee J.H., Yang Y.H., Yu H.X., Canoura J., He J., Li W.Z., Weizmann Y. \& Xiao Y. (2016) Ambient filtration method to rapidly prepare highly conductive, paper-based porous gold films for electrochemical biosensing. Oral presentation, FIU's annual Scholarly Forum during Graduate Student Appreciation Week (GSAW), Florida International University, Miami, FL, March 28 - 29, 2016.

12. Yu H.X., Guntupalli B. \& Xiao Y. (2016) A cooperative-binding split aptamer assay for rapid, specific and ultra-sensitive fluorescence detection of cocaine in 
saliva. Oral presentation, Fifth Annual Forensic Science Symposium, Miami, FL, March $15-16$.

13. Liang P.P., Yu H.X., Guntupalli B. \& Xiao Y. (2015) A paper-based device for rapid visualization of NADH based on dissolution of gold nanoparticles. Poster presentation, 2015 MRS Fall Meeting \& Exhibit, Boston, Massachusetts, November 29 - December 4.

14. Xu X.W., Guntupalli B., Yu H.X. \& Xiao Y. (2014) Target recycling-based assay for rapid and accurate determination of DNA surface coverage on gold nanoparticles. Oral presentation, 2014 MRS Fall Meeting \& Exhibit, Boston, Massachusetts, November 30 - December 5.

15. Guntupalli B., Liang P.P., Dai J.Y., Yang Y.H., He J., Li W.Z. \& Xiao Y. (2013) Ambient filtration method to prepare paper-based electroactive nanoporous gold thin film for detection of dopamine and serotonin. Oral presentation, $89^{\text {th }}$ Florida Annual Meeting and Exposition. Palm Harbor, FL, May $9-11$.

16. Xiao Y., Dai J.Y., Paudyal J., Guntupalli B., Liang P.P., Pubillones F., Yang Y.H., Li W.Z. \& Wang X.T. (2013) Paper-based single-walled carbon nanotube thin film for catalytic detection of nicotinamide adenine dinucleotide in human blood. Invited talk, 89 ${ }^{\text {th }}$ Florida Annual Meeting and Exposition. Palm Harbor, FL, May $9-11$. 